\title{
Structures, functions, and mechanisms of filament forming enzymes: a renaissance of enzyme filamentation
}

\author{
Chad K. Park ${ }^{1}$ (D) - Nancy C. Horton ${ }^{1}$ (D) \\ Received: 23 August 2019 / Accepted: 24 October 2019 /Published online: 16 November 2019 \\ (C) The Author(s) 2019
}

\begin{abstract}
Filament formation by non-cytoskeletal enzymes has been known for decades, yet only relatively recently has its wide-spread role in enzyme regulation and biology come to be appreciated. This comprehensive review summarizes what is known for each enzyme confirmed to form filamentous structures in vitro, and for the many that are known only to form large self-assemblies within cells. For some enzymes, studies describing both the in vitro filamentous structures and cellular self-assembly formation are also known and described. Special attention is paid to the detailed structures of each type of enzyme filament, as well as the roles the structures play in enzyme regulation and in biology. Where it is known or hypothesized, the advantages conferred by enzyme filamentation are reviewed. Finally, the similarities, differences, and comparison to the SgrAI endonuclease system are also highlighted.
\end{abstract}

Keywords Enzyme $\cdot$ Regulation $\cdot$ DNA binding $\cdot$ Nuclease $\cdot$ Run-on oligomerization $\cdot$ Self-association

\section{Introduction}

For over 50 years, it has been known that many enzymes form filamentous structures in vitro as assessed by various biophysical assays, including in some cases imaging by electron microscopy (EM) (Gunning 1965; Kleinschmidt et al. 1969; Olsen et al. 1970; Eisenberg and Reisler 1971; Kemp 1971; Huang and Frieden 1972; Josephs and Borisy 1972; Miller et al. 1974; Frey et al. 1975; Harper 1977b; Harper 1977a; Trujillo and Deal 1977; Meredith and Lane 1978; Zeiri and Reisler 1978; Reinhart and Lardy 1980; Beaty and Lane 1983). However, it was not generally known how filamentation affected enzyme activity. As protein structure determination by $\mathrm{x}$-ray crystallography came to dominate enzyme structure and function studies, enzymes studied tended to be those which produced well-ordered crystals, and filament formation by enzymes seemed nearly forgotten. However, a handful of laboratories continued to work on this

Nancy C. Horton

nchorton@email.arizona.edu

Chad K. Park

ckpark@email.arizona.edu

1 Department of Molecular and Cellular Biology, University of Arizona, Tucson, AZ 85721, USA phenomenon and its role in regulation of their particular enzyme systems (Kessler et al. 1992; Cutler and Somerville 2005; Korennykh et al. 2009; Ingerson-Mahar et al. 2010; Kim et al. 2010; Park et al. 2010). Then, an explosion of interest occurred with the discovery of widespread enzyme self-assembly in cells when viewed by confocal microscopy and with enzymes labeled with fluorescent proteins or antibodies (Narayanaswamy et al. 2009; Werner et al. 2009; Liu 2010; Noree et al. 2010; Ibstedt et al. 2014; Lowe et al. 2014; Suresh et al. 2015; Shen et al. 2016). These screens surprisingly found that many enzymes, not previously appreciated as filamentous, formed large-scale self-assembled structures in cells, including foci, rods, and rings, which are sometimes referred to as cytoophidia. These membraneless, reversible subcellular structures were often seen in response to cellular stress (nutrient starvation, hypoxia) but in many cases, they were also seen under normal physiological conditions (Liu 2010, 2016). Controls with alternative tags, and the use of orthogonal techniques such as mass spectrometry, confirmed that these observations were not merely artifacts of fluorescent labeling such as GFP (Narayanaswamy et al. 2009; Noree et al. 2014; Jin et al. 2017). In addition, several studies investigated the reversibility of the assemblies so as to distinguish from aggregates of misfolded proteins unlikely to represent regulatory states of the enzymes (Narayanaswamy et al. 2009; Suresh et al. 2015). 
Enzymes now shown to form nanoscale filaments in vitro and/or self-assemblies in cells are derived from a diverse array of biochemical and biological pathways, and from diverse cell types including bacteria, yeast, and metazoans (worms, flies, mice, humans). As such, many have medical significance, such as in metabolic diseases, cancer, neurodegenerative disorders, autoimmune disease, and infectious disease. Some have biotechnological or industrial applications, such as in the capture of $\mathrm{CO}_{2}\left(\mathrm{CO}_{2}\right.$ reductase $)$ and production of specialized chemicals and bioremediation (Woodward et al. 2008).

In this review, we attempt to comprehensively collate studies of enzymes found to either form large assemblies in cells (with unknown molecular structures) as well as those with filamentous structures known in atomic or near-atomic detail. For several enzymes, both the molecular structure of the filament is known, at least to low resolutions via electron microscopy, and the cellular self-assemblies have been characterized. We have excluded discussion of cytoskeletal filament forming enzymes, such as actin and tubulin, since these are much better known as filament forming enzymes and have been reviewed extensively elsewhere (Oosawa and Asakura 1975; Bershadsky and Vasil'ev 1988; Kreis and Vale 1999; Aylett et al. 2011). Our particular interest in this phenomenon originated with our studies of SgrAI, a type II restriction endonuclease with unusual allosteric behavior, where binding to one type of DNA sequence results in activation of the enzyme to cleave 14 additional DNA sequences (Bitinaite and Schildkraut 2002). Our investigation into the mechanism responsible for this behavior led to the discovery of filament formation by SgrAI when bound to the activating DNA (which is also a substrate for cleavage of SgrAI known as primary site sequences) (Park et al. 2010; Lyumkis et al. 2013; Ma et al. 2013). The filamentous form recruits additional copies of SgrAI bound to the second type of DNA sequence (secondary sites) (Park et al. 2010; Shah et al. 2015). The filamentous state preferentially stabilizes the activated conformation of the enzyme; hence, SgrAI in the filament is activated for DNA cleavage (Polley et al. 2019). In reviewing the literature for precedence of this type of behavior, we discovered that such phenomenon was also under investigation in the regulation of IRE1 (the unfolded protein response kinase/ribonuclease) (Korennykh et al. 2009), cytosine triphosphate (CTP) synthase (Ingerson-Mahar et al. 2010; Liu 2010; Noree et al. 2010; Carcamo et al. 2011; Chen et al. 2011), and acetyl CoA carboxylase (ACC) (Kim et al. 2010). Concurrently, the publication of proteome-wide screens for self-assemblies in cells, as well as older literature showing filamentation by a number of metabolic enzymes, became relevant to our studies and are also covered in this review.

Several other reviews have been published recently regarding enzyme filamentation (Cabeen and Jacobs-Wagner 2010; Liu 2010; Barry and Gitai 2011; Griffin and Gerrard 2012; O'Connell et al. 2012; Selwood and Jaffe 2012; Carcamo et al.
2014; Shen et al. 2016; Garcia-Seisdedos et al. 2017; Prouteau and Loewith 2018). We focus here in this review on structurefunction studies of filament forming enzymes, while also attempting to provide an up-to-date, comprehensive listing of enzymes known to form cytoophidia or intracellular selfassemblies. We attempt to find similarities and differences in the structures and mechanisms, and are particularly interested in why filament formation is necessary in addition to more "traditional" enzyme regulatory mechanisms. Our own studies with SgrAI indicate that filament formation provides for a much faster activation of the enzyme (Barahona et al. 2019). We also find that due to a particularity of the enzyme kinetic pathway, namely a relatively slow, rate limiting second order association rate constant for filament assembly, that filament formation can provide a means to sequester enzyme activity on only particular substrates of interest, those with high local concentrations (Park et al. 2018a, b; Barahona et al. 2019). This could be a general phenomenon for enzymes that have more than one class of substrate and require regulation of when and where that secondary substrate activity will occur. However, it remains to be seen for the vast majority of enzymes known to filament, what advantage filamentation has towards enzyme function and/or regulation. What is known in general, considering all enzymes reviewed herein, is that filamentation can occur through linear polymer assembly or more commonly helical assembly (left or right-handed). The filament form may either be the more active form, or the less active form, or may have altered activity (substrate preference or even a completely different type of activity). The purpose of filamentation may be to perform a structural function, such as determining cell shape, or may form a scaffold for the binding of other proteins. As such, it can sometimes perform functions in signaling. Filament formation can be responsive to cellular conditions, thereby regulating enzyme activity and any other activity such as signaling or chaperone function. All in all, enzyme filamentation has been found to perform many different functions in cells, and we are likely to continue to discover new roles and functions for this interesting phenomenon.

\section{Structurally characterized enzyme filaments}

\section{Acetyl CoA carboxylase}

Acetyl CoA carboxylase (ACC) has a central role in primary metabolism, and its upregulation is linked to obesity related diseases (Harwood 2004; Tong 2013; Stiede et al. 2017) and tumor growth (Swinnen et al. 2006; Svensson et al. 2016; Guri et al. 2017). This enzyme catalyzes the carboxylation of acetyl-CoA to malonyl-CoA, the first and rate-limiting step of fatty acid biosynthesis. The ACC enzyme uses biotin as a carboxyl carrier, requires adenosine triphosphate (ATP), and uses bicarbonate as a carbon donor (Abu-Elheiga et al. 1995; 
Saggerson 2008; Wakil and Abu-Elheiga 2009) (Fig. 1a). Mammals contain two isoforms, ACC1 and ACC2 (AbuElheiga et al. 1995). While both isoforms produce malonyl$\mathrm{CoA}, \mathrm{ACC} 1$ is found in lipogenic tissues, is predominantly cytosolic, and generates malonyl-CoA that is used for fatty acid synthesis. ACC2 is mitochondrial and is found mostly in oxidative tissues, such as heart and skeletal muscle where it regulates fatty acid beta-oxidation because its product, malonyl-CoA, is a negative regulator. Hence, ACC2 has been identified as a potential target for treating metabolic

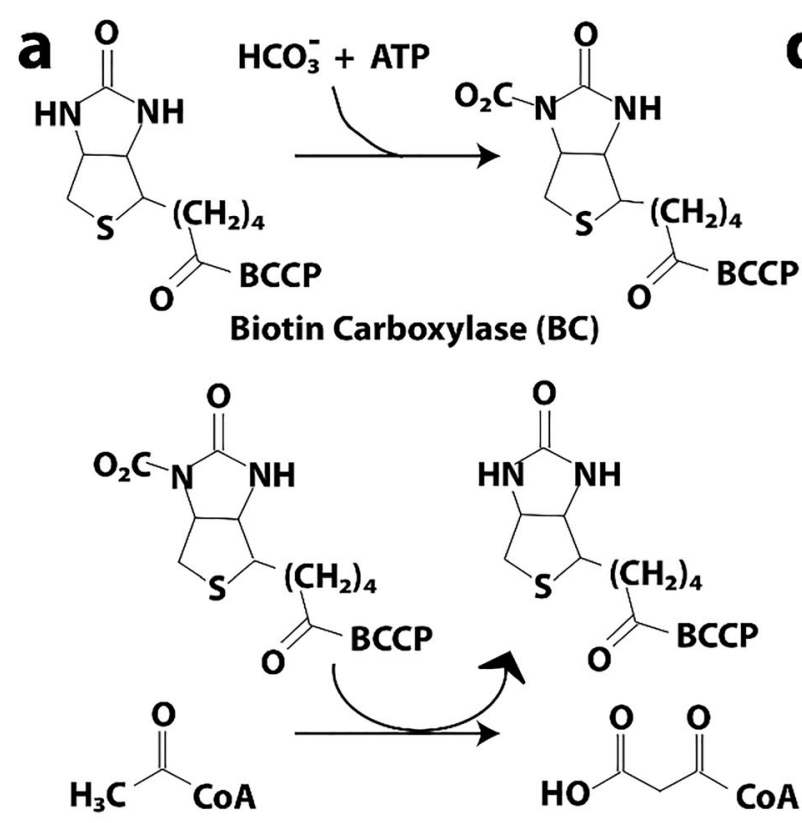

b

Carboxyltransferase (CT)

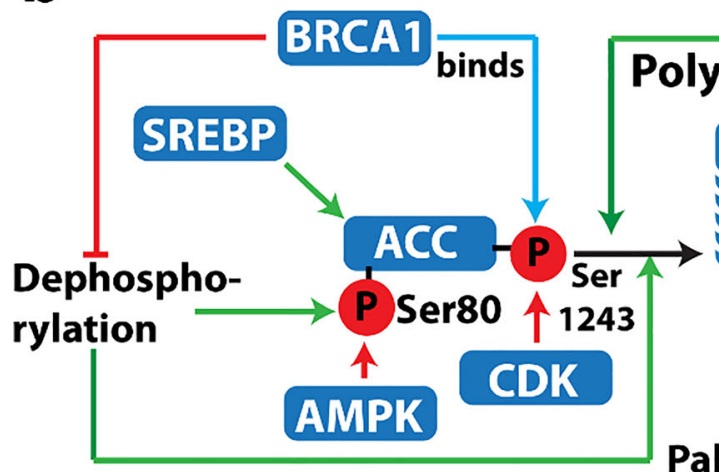

Palmitoyl-CoA

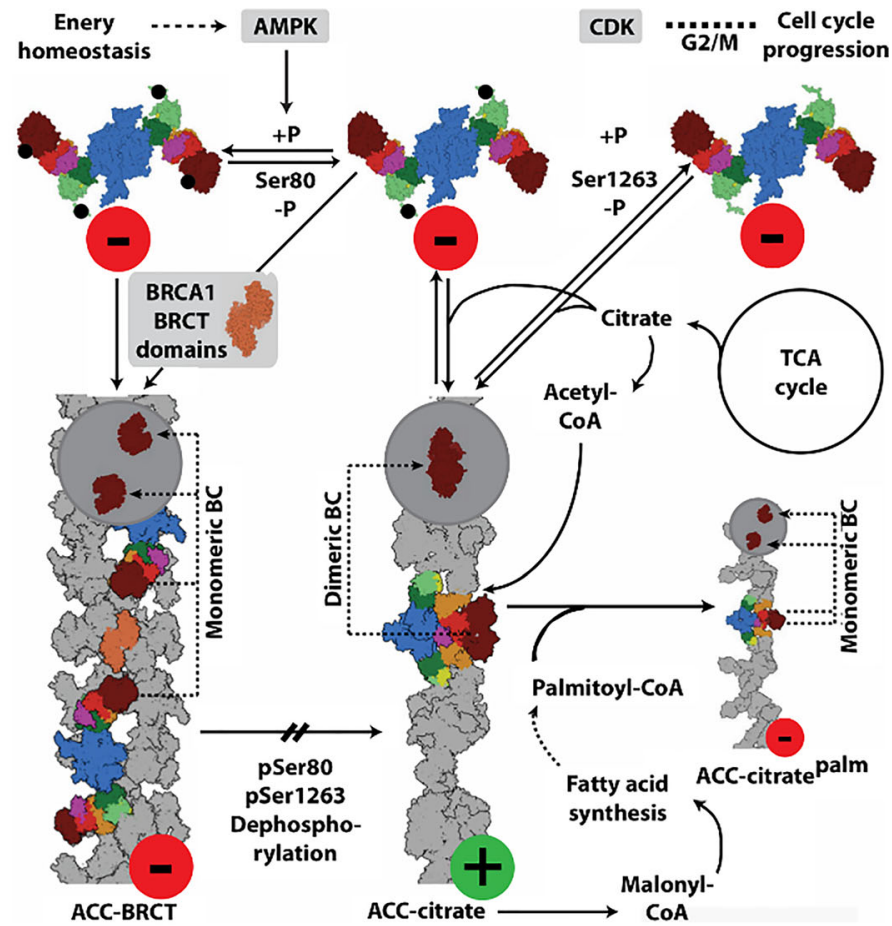

ACC-BRCT

CoA

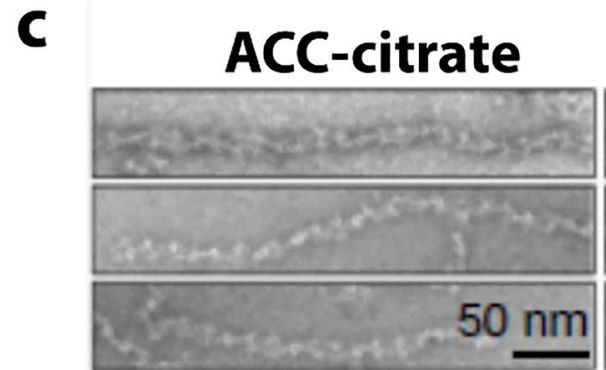

Fig. 1 Filamentation of acetyl-CoA carboxylase (ACC). a Reactions catalyzed by ACC. b Complex regulation of ACC function involving filamentation (a.k.a. polymerization), phosphorylation, BRCA1 binding, and allosteric inhibition (adapted with permission from Hunkeler et al. 2018). c Negative stain electron micrographs of three different filament forms of ACC (adapted with permission from Hunkeler et al. 2018). d
Roles of the three filament forms in regulating ACC activity (indicated by red or green circles) (adapted with permission from Hunkeler et al. 2018). d GFP labeled ACC form cytoophidia (large self-assemblies) in yeast cells under nutrient starvation (adapted with permission from Shen et al. 2016) 
syndromes because knockout of ACC2 reduces fat content and increases the resistance to high fat/high carbohydrateinduced obesity and diabetes (Abu-Elheiga et al. 1997, 2001, 2003).

Mammalian ACCs are typically homodimeric, but can also assemble into polymers with increased activity ( $\sim 60$-fold) (Meredith and Lane 1978; Beaty and Lane 1983). ACCs are regulated by a complex interplay of phosphorylation, binding of allosteric regulators and protein-protein interactions, which is further linked to filament formation (Vagelos et al. 1962; Kleinschmidt et al. 1969; Moss and Lane 1972; Ha et al. 1994; Brownsey et al. 2006; Tong 2013) (Fig. 1b). These filaments were discovered 50 years ago (Kleinschmidt et al. 1969; Meredith and Lane 1978; Ashcraft et al. 1980) but only more recently were they shown to be relevant to in vivo activity and to be characterized structurally (Kim et al. 2010). Polymerization of ACC1 increases enzymatic activity and is induced in vitro by supraphysiological concentrations of citrate (> $5 \mathrm{mM}$ ) (Meredith and Lane 1978, Beaty and Lane 1983). Citrate is produced in the mitochondria and is used as a substrate by ATP citrate lyase, which converts citrate to acetyl-CoA, the substrate of ACC.

Filament formation is also modulated by other proteins. For the mitochondrial isoform of ACC (ACC2), midline-1-G12like-interacting protein (MIG12) binding alone is sufficient to drive polymerization (Kim et al. 2010). The presence of MIG12 further reduces the concentration of citrate needed for polymerization fivefold for the cytoplasmic isoform ACC1 (to $<1 \mathrm{mM}$ ), and also increases the activity by $\sim 60$ fold (Kim et al. 2010). However, Spot14, a paralog of MIG12, forms assemblies with MIG12 which attenuates activation and filament formation by both ACC1 and ACC2 (Park et al. 2013).

A recent structural investigation showed the formation of three different types of filaments of human ACC1 (Hunkeler et al. 2018) (see Fig. 1c). The authors used cryo-electron microscopy and single particle reconstruction to reveal filaments at 4-6 ̊ resolution, allowing the fitting of previously determined crystal structures into the cryo-EM envelopes. The basis of activation or inactivation in the filaments resulted from the locking in of either an active or inactive conformation, compared to non-filamentous ACC1 that was shown to populate both active and inactive conformations. One type of filament is activated, due to the dimerization of its two biotin carboxylase (BC) domains (which is required for activity), while the other two show these domains are not in close proximity, and are inactive.

The active filament (ACC-citrate) is formed in the presence of citrate (at $10 \mathrm{mM}$ ), and only with a form of ACC1 that is free of phosphorylation. The filament is a run-on oligomer, as it is composed of a linear polymer of ACC1 dimers which appear to add singly at either end. The filament is a lefthanded helix, with three ACC1 dimers per turn and a rise (distance between adjacent ACC1 dimers in the filament) of $154 \AA$. In the active filament, not only are the $\mathrm{BC}$ domains dimerized, but also the $\mathrm{BCCP}$ (biotin carrier protein domain) is in a position where it may rotate to carry the biotin from the $\mathrm{BC}$ to the $\mathrm{CT}$ (carboxyl transferase) domain (Fig. 1d). These filaments can be quite long at $0.5-1.0$ microns in length.

A second filamentous form of ACC1 is induced by the addition of a tenfold molar excess of the feedback inhibitor palmitoyl-CoA to the filament form of ACC1 induced by citrate. This form, ACC-citrate ${ }^{\text {palm }}$ is presumably inactive, as the $\mathrm{BC}$ domains are no longer dimerized. The helical backbone is thinner from which the $\mathrm{BC}$ domains now protrude, although the filament still shows 3 copies of the ACC1 dimer per helical turn (left-handed) (see Fig. 1b).

A third type of filament results from binding of the BRCT domains of BRCA1 to a phosphorylated form of ACC1 (ACC-BRCT). Phosphorylation at Ser78, Ser80, Ser1201, and Ser1216 are known to inactivate human ACC1, with Ser80 and Ser1201 phosphorylation having the greatest effect (Ha et al. 1994). Mutations in the BRCT domains eliminating BRCA1 binding to ACC result in elevated lipogenesis, which is a prerequisite for cancer cell growth (Magnard et al. 2002; Ray et al. 2009). The C-terminal tandem BRCT domains of BRCA1 bind ACC1 by recognizing phosphorylated Ser1263, a residue that is phosphorylated in a cell cycle-dependent manner, and this binding inhibits dephosphorylation of Ser80 of ACC1 (Magnard et al. 2002; Shen and Tong 2008; Ray et al. 2009). This filament is distinct in that it has a twostranded appearance, with a rise of $190 \AA$ between adjacent ACC1 dimers. However, it is also a left-handed filament with $3 \mathrm{ACC} 1$ dimers per turn. The BRCT domains laterally "decorate" the filament and interlink adjacent $\mathrm{ACC} 1$ dimers. Like the ACC-citrate ${ }^{\text {palm }}$ filament, the $\mathrm{BC}$ domains are monomeric and the BCCP domains appear to be sterically unable to reach any of the active sites; hence, the ACC1 in this form is predicted to be enzymatically inactive.

Large assemblies (i.e., cytoophidia) of ACC are seen in screens under conditions of nutrient starvation (in stationary phase) (Fig. 1e) (Shen et al. 2016). These are much larger than the filaments viewed by EM (Fig. 1c), but may be made of bundles of likely inactivated enzymes.

\section{Phosphofructokinase}

Phosphofructokinase (PFK) is an important metabolic enzyme that acts as a gatekeeper since it unidirectionally catalyzes the committed step of glycolysis, namely the conversion of fructose 6-phosphate (F6P) to fructose 1,6-phosphate (F16P) (Fig. 2a). Glycolysis is the core of central carbon metabolism; its intermediates provide precursors important for generating ATP through glucose oxidation, serine for one-carbon metabolism, sugars for protein glycosylation, and building blocks for nucleotide synthesis through the pentose phosphate shunt. 


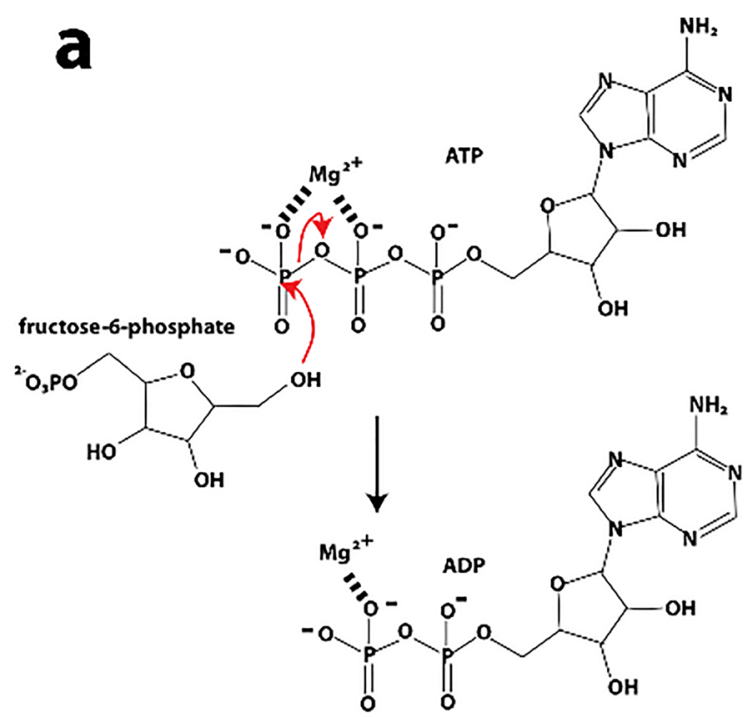

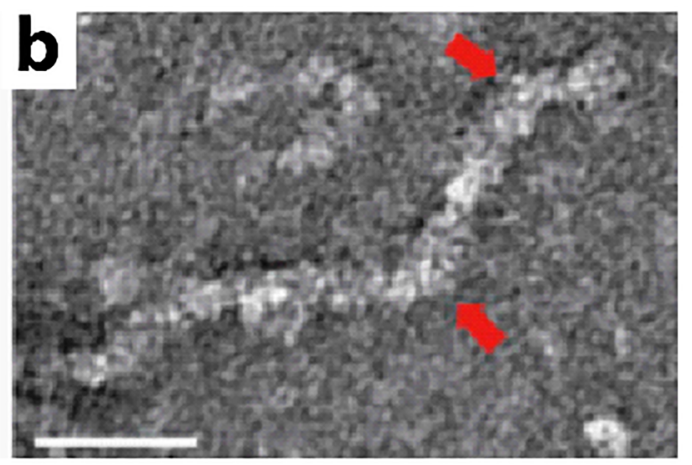

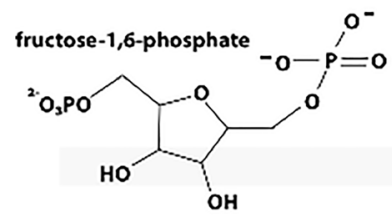
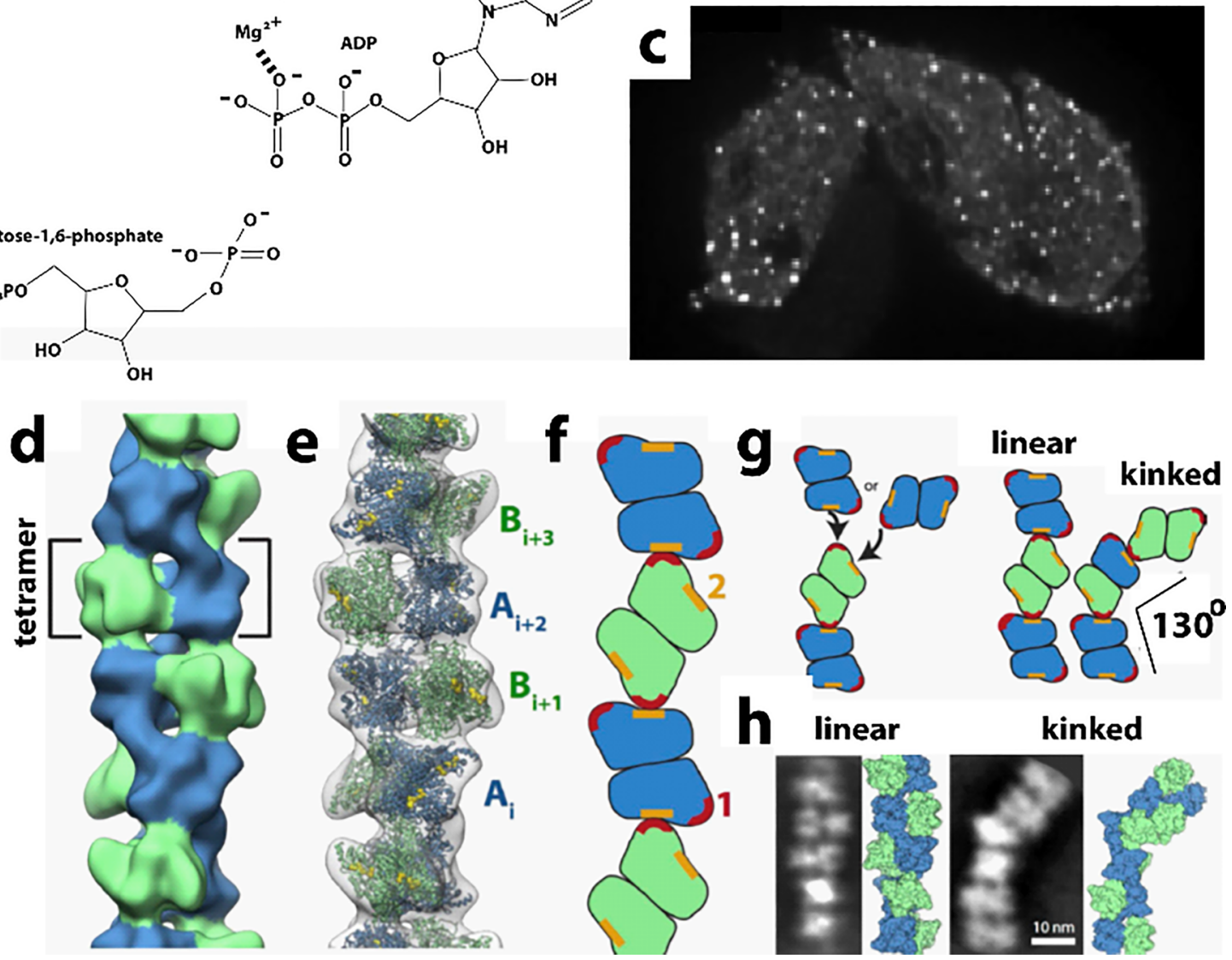

Fig. 2 Reactions and filament formation by phosphofructokinase-1 (PFK). a Reaction catalyzed by PFK (EC 2.7.1.11). b Negative stain EM image of PFKL (liver isoform of human PFK) filament. Bar represents $100 \mathrm{~nm}$. Arrows identify "kinks" (adapted with permission from Webb et al. 2017). c PFKL-EGFP filaments in cells MTln3 cells following addition of $10 \mathrm{mM}$ citrate using confocal microscopy showing punctae. No such puncta were apparent before the addition of citrate (not shown) (adapted with permission from Webb et al. 2017). d Threedimensional reconstruction of PFKL filament (adapted with permission from Webb et al. 2017). e Coordinates of the tetramer of the PFKP crystal

Due its importance in cellular metabolism, it is highly regulated with allosteric regulation by over 10 metabolites to allow cells to fine tune their energy needs. PFK from yeast, C. elegans, and human liver have been shown to form filaments, fibers, and/or punctate foci (Jang et al. 2016; Shen et al. 2016; Webb et al. 2017). The in vitro self-association of

structure fit into the three-dimensional reconstruction (adapted with permission from Webb et al. 2017). f Schematic of PFKL tetramer associations in PFKL filament (adapted with permission from Webb et al. 2017). g Left, schematic showing two different associations of PFKL tetramers leading to either linear or kinked filaments. Right, schematic of linear and kinked filaments (adapted with permission from Webb et al. 2017). h Left images, 2D class averages and right images, reconstructions, for linear and kinked filaments (adapted with permission from Webb et al. 2017)

purified rabbit liver PFK has been known since at least 1971, using size exclusion chromatography (Kemp 1971). Follow-up studies using sedimentation, analytical ultracentrifugation, and fluorescence anisotropy also showed PFKL selfassemblies (Trujillo and Deal 1977; Reinhart and Lardy 1980). Finally, negative stain EM performed in 1980 showed 
these to be composed of filaments (Foe and Trujillo 1980), which was further investigated recently (Fig. 2b) (Webb et al. 2017). Filament formation occurs with starvation in yeast (Shen et al. 2016), stress from increased activity and/or hypoxia in neurons of $C$. elegans (Jang et al. 2016), and high concentrations of citrate or its substrate F6P in human PFKL (Fig. 2c) (Webb et al. 2017), suggesting a role in enzyme regulation. However, the effect of filamentation on enzyme function are not currently known. Dysregulation of PFK activity has implications for cancer (Hanahan and Weinberg 2011; Moreno-Sanchez et al. 2012; Yi et al. 2012; Webb et al. 2015), neuronal dysfunction (Jang et al. 2016), type II diabetes (Ristow et al. 1999a, b) and autoimmune disease (Yang et al. 2015). A low-resolution structure of the filament formed by human PFKL has been reported using single particle reconstruction and negative stain TEM (Fig. 2d) (Webb et al. 2017). No high-resolution structures or structures of PFK filaments are known, though crystal structures of non-filamentous PFK have been reported (Bruser et al. 2012; Schoneberg et al. 2013; Kloos et al. 2014; Kloos et al. 2015; Webb et al. 2015). Fitting of the non-filamentous structure into the low-resolution EM envelope of the PFKL filament shows a right-handed helix with stacked PFKL tetramers related by a rotation of $221^{\circ}$ and translation of $83 \AA$ between subunits (Fig. 2e) (Webb et al. 2017). The average size of filaments observed by EM is 6 tetramers, although filaments of 11 or more were also observed (Webb et al. 2017). Filaments were observed to be kinked at random locations (Fig. 2b), and structural analysis revealed that for every addition of PFKL tetramer, two possible interfaces are possible. One interface leads to an unkinked junction, the other to a kinked junction of $\sim 130^{\circ}$ (Fig. 2f-h) (Webb et al. 2017). In vivo, TIRF was used to show punctate foci of PFKL in cells and indicated that filaments must be smaller than 15 tetramers in length, consistent with the EM results (Fig. 2b) (Webb et al. 2017).

No detailed kinetic analyses of the role of filament formation in PFK function have been reported. Therefore, the following questions remain unanswered: how does filament formation affect PFKL enzyme activity? It's stimulation by its substrate suggests activation, however it is also stimulated by the inhibitory effector citrate. Also, how do its 10 allosteric effectors affect filament formation and PFKL activity? Allosteric effectors include: ATP (a required reactant but also an allosteric inhibitor at higher concentrations), ADP (a product of the reaction, but also an allosteric activator), fructose 2,6-phosphate (activator) and citrate (inhibitor), among many others. Further, what advantages does filament formation confer to PFKL function?

\section{Glucokinase}

Glucokinase (Glk1) is found in fungi, and one of three kinases in S. cerevisiae that initiate glycolysis by phosphorylating glucose in an ATP-dependent reaction (Fig. 3), and also possesses an actin fold. The enzyme is induced in the absence of glucose and expressed in high glucose (Maitra 1970). A fusion protein of Glk1 and GFP is found to be diffused throughout the cytoplasm in the absence of glucose, but formed fiberlike self-assemblies in the presence of glucose (Stoddard et al. 2019). These bundles rapidly disassemble when glucose was removed from the growth media (Stoddard et al. 2019). Purified Glk1 at $7.5 \mu \mathrm{M}$ is non-filamentous in the absence of ligands, but was found to form helical filaments in vitro in the presence of its substrates (ATP and glucose, mannose, glucosamine) or products (ADP and sugar-6-phosphate) (Stoddard et al. 2019). No filaments were formed in response to $\mathrm{pH}$, fructose, or galactose (fructose and galactose result in fiber formation in vivo) (Stoddard et al. 2019). Some polymerization is found with $\mathrm{N}$-acetylglucosamine-6-phosphate and $\mathrm{N}$-acetylglucosamine, inhibitors of Glk1 (Stoddard et al. 2019).

Polymerization of Glk1 in vitro was found to occur with a critical concentration of $2 \mu \mathrm{M}$, below which the enzyme remains unpolymerized and active, but above this concentration, the enzyme forms polymers with little to no activity (Stoddard et al. 2019). Therefore, below $2 \mu \mathrm{M}$, product formation increases with enzyme concentration; however, above $2 \mu \mathrm{M}$, the rate of product formation is independent of enzyme concentration. Above $2 \mu \mathrm{M}$, the concentration of non-polymerized enzyme remains constant while the concentration of enzyme in the filaments increases with increasing overall enzyme concentration. This, in effect, results in an upper limit to the enzyme activity, which may play a role in protecting the cell against excess toxic enzyme activity. Assembly of filaments was also found to be rapid, reaching the steady-state equilibrium in a matter of seconds (Stoddard et al. 2019).

The filament structure was determined using cryo EM (Fig. 3d) and found to be an anti-parallel double stranded, righthanded helix (Stoddard et al. 2019). The enzyme is confirmed to be in the closed state, and evidence of ATP binding in the active site is found (Stoddard et al. 2019). Each enzyme copy makes extensive contacts to enzyme copies before and after in the same filament, as well as the copy opposite it in the other

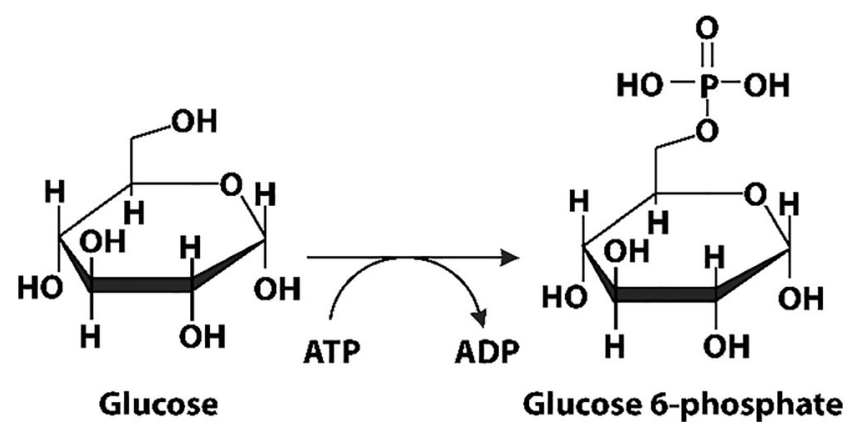

Fig. 3 Reaction catalyzed by glucokinase (Glk1), phosphorylation of glucose using ATP 
strand (Stoddard et al. 2019). The contacts between enzymes are distinct from those found in actin, and evolutionary considerations indicate that polymerization by Glk1 evolved independently from that of actin (Stoddard et al. 2019). Being an anti-parallel, double helical filament, it has no polarity and one end is equivalent chemically to the other. No larger bundles of the dimensions formed in vivo were found in vitro, indicating that those larger self-assemblies in cells are the result of filaments assembling due to crowding, filament binding proteins, or even possibly due to the GFP tag.

Polymerization was found to be important for cell fitness, as mutants that eliminate filamentation without affecting the enzymatic activity of non-polymerized Glk1 show reduced survival compared to wild type (Stoddard et al. 2019). Protecting the cell from toxic excess sugar kinase activity is important, and Glk1, unlike the two other similar kinases Hxk1 and Hxk2, which do not polymerize, is not inhibited by trehalose-6-phosphate (a metabolic intermediate that transiently accumulates as a result of elevated glucose 6phosphate) (Stoddard et al. 2019). Glk1 polymerization has also been argued to have evolved as a strategy to allow cells to adapt to environmental transitions occurring faster than the cell can change its protein levels (Stoddard et al. 2019).

\section{Cytosine triphosphate synthetase}

CTP synthase (CTPS) is a universally conserved enzyme that forms filaments in vitro and rod-shaped self-assemblies in cells, both the bacterial and eukaryotic cells. This enzyme is a focal point for regulating pyrimidine levels through its formation of CTP from UTP (Fig. 4a) and is a target for cancer, immunosuppressive, and antiparasitic drugs (Hindenburg et al. 1985; Kang et al. 1989; Politi et al. 1995; Hofer et al. 2001; Martin et al. 2014). Cytoophidia, or large rod-like selfassemblies, were seen with GFP labeled CTPS in yeast under conditions of nutrient starvation (Noree et al. 2010) (Fig. 4b). Such structures were also discovered in the bacteria Caulobacter crescentus (Ingerson-Mahar et al. 2010) and E. coli (Barry et al. 2014). Filament formation by the bacterial enzyme inhibits its enzymatic activity, is required for nucleotide homeostasis, and its disruption significantly affects cell growth and metabolism (Aughey et al. 2014; Barry et al. 2014). In bacteria, the product (CTP) drives filamentation, which may also have a role in cell shape (Ingerson-Mahar et al. 2010). The filaments are also sensitive to the balance of substrate and product and can rapidly dissociate into active tetramers in response to changes in substrate concentration (Calise et al. 2014; Lynch et al. 2017). These dynamics may act to buffer the catalytic activity of CTPS (Aughey et al. 2014). A negative stain EM image of filaments formed by a bacterial CTPS is shown in Fig. 4c.

Human CTPS, in contrast to the bacterial, forms filaments with increased enzymatic activity, and an image of negative stain EM is shown in Fig. 4d (Lynch et al. 2017). The bacterial and human CTPS filaments also differ considerably in overall filament architecture and conformation of the enzyme (Lynch et al. 2017). Filament formation by human CTPS is favored in the presence of substrates UTP and ATP, but not the products, CTP and ADP (Lynch et al. 2017). Filaments assembled with substrate are disassembled upon addition of glutamine presumably due to accumulation of CTP, but using nonhydrolyzable AMPPNP stabilizes filaments against disassembly (Lynch et al. 2017). In eukaryotes, cytoophidia and filament formation is part of a stress response and varies with developmental state in some organisms (Carcamo et al. 2011; Aughey et al. 2014; Calise et al. 2014; Noree et al. 2014; Petrovska et al. 2014; Strochlic et al. 2014). CTPS in Drosophila germ cells form cytoophidia at developmental stages with a high demand for CTP (Strochlic et al. 2014). CTPS activity is regulated by phosphorylation (Choi et al. 2003; Han et al. 2005; Chang et al. 2007), but it is not known if phosphorylation has an effect on polymerization. CTPS cytoophidia colocalize with other metabolic and signaling enzymes too, raising the possibility that other enzymes may regulate CTPS filament formation and/or be incorporated into the filaments (Liu 2016).

The homotetrameric structure of CTPS is conserved between eukaryotes and bacteria (Goto et al. 2004; Endrizzi et al. 2005; Kursula et al. 2006; Lauritsen et al. 2011). Each monomer is composed of a glutamine amidotransferase doman (GAT) fused to kinase-like ammonia ligase domain (AL) via an alpha helix linker. Ammonia from GAT is transferred to AL, and ligated to UTP forming CTP in an ATP hydrolysis-dependent reaction (Endrizzi et al. 2005). The 4.6 $\AA$ cryo-EM reconstruction of the bacterial (E. coli) CTPS (ecCTPS) filament (Fig. 4e, right) with bound inhibitor CTP shows that it is formed from stacked tetramers that interact through the GAT \& linker domains (Lynch et al. 2017). These authors also solved a crystal structure of ecCTPS in a non-filamentous form with bound CTP and found the same conformation of the enzyme and CTP binding. This result is significant in that it supports an idea that the filament locks in an inhibitory conformation of the enzyme, and that CTP binding allosterically controls ecCTPS and filament formation by inducing a filament competent conformation in the enzyme. Notably, a mutation associated with drug resistance in CTPS, E155K, eliminates the ability to form filaments (Trudel et al. 1984; Barry et al. 2014). Significantly, the mutant CTPS lacks the large reduction in activity found with wild type upon filament formation, and binds CTP less tightly. These results indicate a role for filament formation in providing an added layer of regulation in the form of rapid and efficient inhibition.

To further investigate the mechanism of inhibition of ecCTPS via filament formation, disulfides were engineered to create a filament that could be formed without the addition of product nucleotides (i.e., CTP, ADP) (Fig. 4c), and it was 

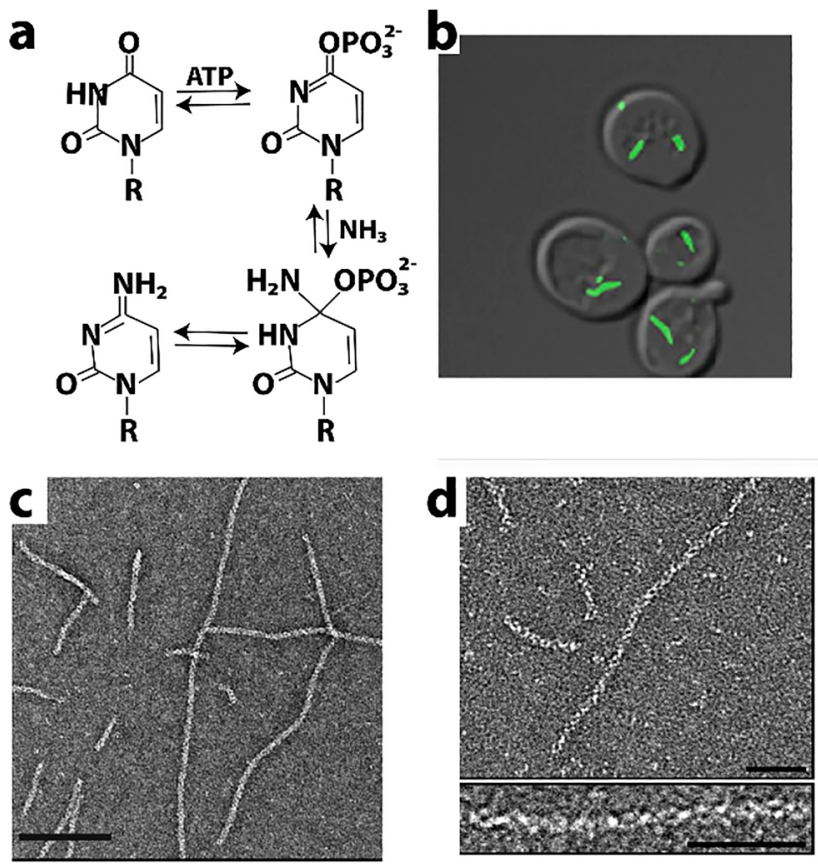

Fig. 4 Reaction catalyzed by, and filaments formed of, CTP Synthase (CTPS). a Reaction catalyzed by CTPS (EC 6.3.4.2). b Cytoophidia or large rod-like structures of GFP labeled self-assemblies observed in yeast under nutrient starvation conditions (adapted with permission from Noree et al. 2010). c Filaments of ecCTPS formed by a mutant designed to for crosslinks to stabilize the filamentous structure viewed by negative stain EM. Line represents $200 \mathrm{~nm}$ (adapted with permission from Lynch et al.

found to have a fivefold reduction in activity, consistent with the filament directly inhibiting activity. Products soaked into these filaments bind, as observed by cryo-EM; hence, the filament structure is not occluding CTP or ADP binding sites. However, when repeated with substrates UTP and ATP, no binding was seen. It was concluded that the filament allosterically regulates ecCTPS activity by stabilizing an intrinsically lower-activity state upon incorporation into filaments, independent of CTP binding (Lynch et al. 2017).

The structure of the filament of human CTPS has been determined to $6.1 \AA$ by cryo-EM (Fig. 4e). These form in the presence of substrates UTP and ATP (and the allosteric effector GTP), but not CTP and ADP products, exactly the opposite of ecCTPS (Lynch et al. 2017). The structure also shows stacked tetramers, however with different assembly contacts, tetramer interfaces, and protomer conformations than the bacterial CTPS filament (Lynch et al. 2017). Filament assembly is mediated primarily via an insertion in the GAT domain that appeared early in eukaryotic evolution. But like ecCTPS, filament formation is driven by binding to allosteric effectors, however with the opposite result on activity, enzyme conformation, and with different filament architectures (Fig. 4e). A mutation in the GAT domain insert that mediates filament interactions between tetramers, H355A, forms the native tetramer but not the

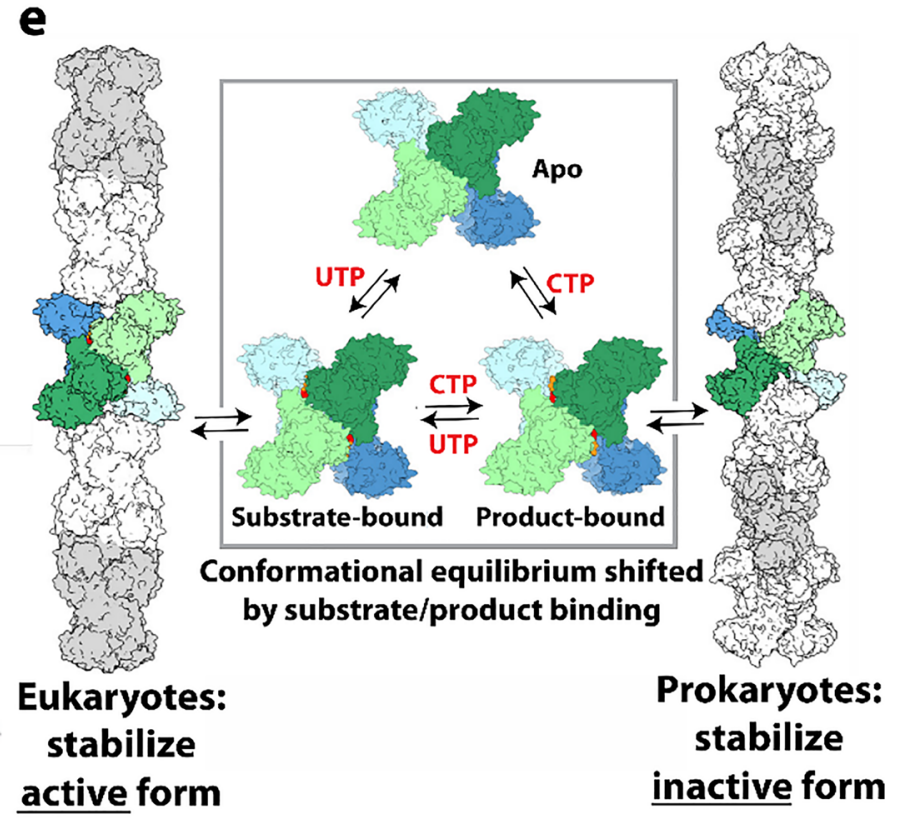

2017). d Filaments of human CTPS formed in the presence of UTP, ATP, and GTP. Bar represents $50 \mathrm{~nm}$ (adapted with permission from Lynch et al. 2017). e Models of CTPS filaments from human (left) and bacteria (right) based on single particle cryo-EM reconstructions and their relationship to the presence of substrates (UTP) and products (CTP) (adapted with permission from Lynch et al. 2017)

filament, and shows a 6-fold reduction in activity. This is consistent with the filament having the function of holding the enzyme in an active conformation. The active conformation appears to be the result of a $10^{\circ}$ rotation between the GAT and AL domains, relative to all previously reported structures, which results in a tunnel that could be used for ammonia transfer between the two active sites. The rotation is connected to filament assembly since it is also necessary for positioning the GAT domains for interactions between CTPS tetramers in the filament.

Both types of filament appear to have $~ 3-4$ CTPS tetramers per turn (Fig. 4e). The ecCTPS associates via interdigitation of stacked Xs (each X is a CTPS tetramer), while hCTPS associates by Xs interacting at their "tips." The "X" is a little different in the two as well, as it is somewhat elongated in one direction (that which coincides with the filament axis) and compressed in the other (by 5-6 $\AA$ each). Both polymerize via their GAT domains. The short helical insertion in human CTPS provides the primary assembly interface and also prevents the formation of ecCTPS-like contacts (Lynch et al. 2017).

What remains unclear is the relative advantage of stabilizing hCTPS in an active conformation in the filaments. It could be to keep the bulk of the enzyme under conditions of cellular stress in a state primed for maximal activity immediately on return to normal growth conditions. 


\section{Inositol monophosphate dehydrogenase}

The precise regulation of nucleotide biosynthesis is crucial for normal cell metabolism (Rudolph 1994). Like CTPS, which forms the rate limiting metabolite in the de novo synthesis of CTP, inositol monophosphate dehydrogenase (IMPDH) catalyzes the rate limiting step for the synthesis of GTP (Fig. 5a). The substrate of IMPDH, inositol monophosphate (IMP), is generated by a large complex of related enzymes called the purinosome (An et al. 2008; Deng et al. 2012). However, IMPDH is not part of the purinisome, perhaps why it forms super structures of its own. IMPDH is an important drug target (antiviral, antiparasitic, antileukemic, antibacterial, and immunosuppressive (Ratcliffe 2006, Chen and Pankiewicz 2007, Nair et al. 2007, Nair and Shu 2007, Hedstrom 2009)) because inhibition causes not only a reduction of the guanine nucleotides pools, but also more importantly an imbalance between adenine and guanine nucleotides, leading to wide-ranging repercussions (Hedstrom 2009). Humans code for two differentially expressed IMPDH genes (IMPDH1 and IMPDH2) (Carr et al. 1993), and both have been shown to assemble into the large rod- and ring-shaped assemblies visible in cells as cytoophidia (Fig. 5b) (Gunter et al. 2008; Thomas et al. 2012). In Drosophila, IMPDH has also been shown to bind to DNA and repress transcription of histone and E2F genes (Kozhevnikova et al. 2012). E2F is critical for the G1/S transition and DNA replication; hence, this activity of IMPDH would result in slowing the cell cycle. In contrast, the enzymatic activity of IMPDH is under higher demand in proliferating cells.
Fig. 5 Reaction catalyzed and filaments formed by IMPDH. a Reaction catalyzed by IMPDH (EC 1.1.1.205) (adapted with permission from Hedstrom 2009). b Cytoophidia, or large rod and ring like structures, formed in HEK293 cells by GFP labeled IMPDH after introduction of the IMPDH inhibitor ribavirin (adapted with permission from Anthony et al. 2017). c Negative stain EM micrograph of filamentous IMPDH induced with purified IMPDH in vitro with $5 \mathrm{mM} \mathrm{NAD}^{+}$and $1 \mathrm{mM}$ ATP (adapted with permission from Anthony et al. 2017). The scale bar represents $50 \mathrm{~nm}$. d Single particle reconstructions using cryo-EM images of filaments of IMPDH formed with either ATP and $\mathrm{NAD}^{+}$(left) or ATP and GTP (right) (adapted with permission from Anthony et al. 2017). e Model relating open (left) and collapsed (right) filaments of IMPDH (adapted with permission from Anthony et al. 2017) a 1. Oxidation of IMP

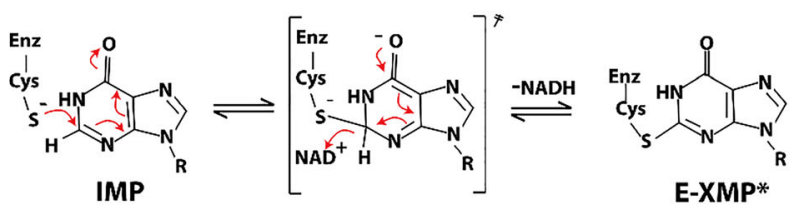

2. Hydrolysis of E-XMP*

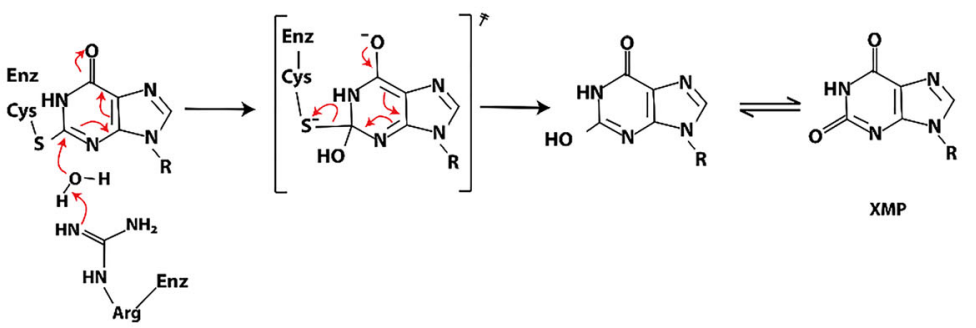

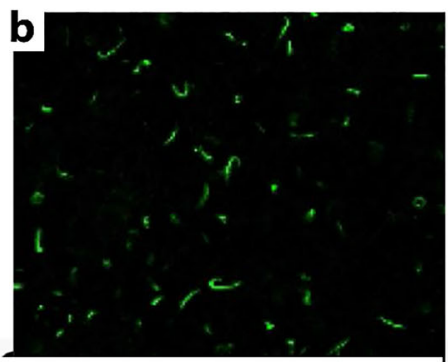

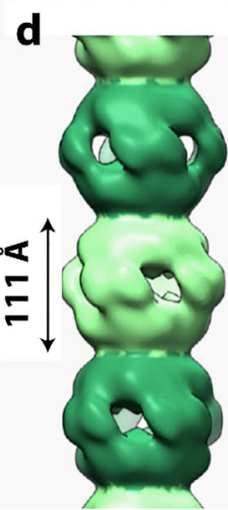

ATP, NAD+

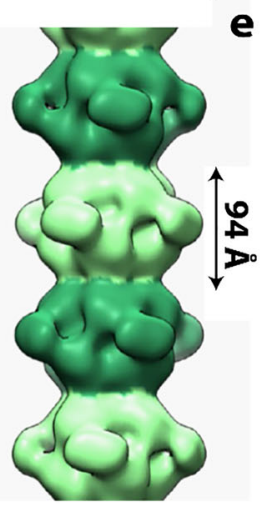

ATP, GTP

e
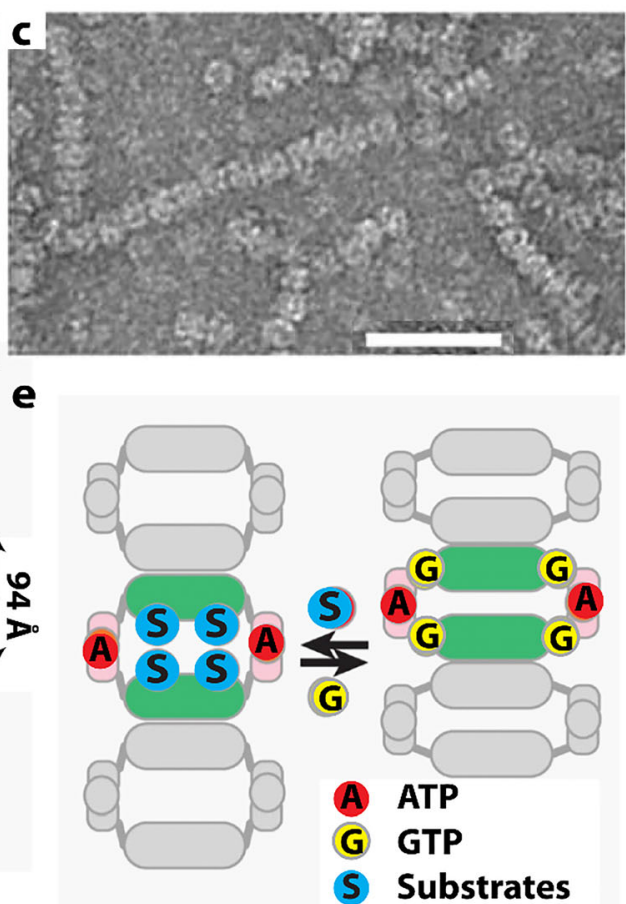
IMPDH has been shown to form cytoophidia in vivo in mammalian cells as well as in Drosophila (Ji et al. 2006; Carcamo et al. 2011; Liu 2016). CTPS also forms these types of structures, and cytoophidia of CTPS and IMPDH are often are observed at the same time, often colocalizing but other times forming separate structures (Carcamo et al. 2011; Chang et al. 2018; Keppeke et al. 2018). CTPS and IMPDH cytoophidia have also been showed to be independent but related structures (Keppeke et al. 2018). The sizes and locations can also be very different for the two types of assemblies, even in the same cell (Keppeke et al. 2015). There is also currently no evidence that these two enzymes form mixed filaments.

IMPDH cytoophidia are found to occur spontaneously in a number of cell types, but can also be induced (Carcamo et al. 2014; Chang et al. 2015). IMPDH cytoophidia assembly is promoted by its substrate IMP (Keppeke et al. 2018). They form spontaneously in mouse pancreatic islet cells in response to nutrient uptake (Chang et al. 2015). Formation of the IMPDH cytoophidia in mouse embryonic as well as induced pluripotent stem cells is correlated with rapid cell proliferation (Carcamo et al. 2011; Keppeke et al. 2018). When cells launch division, they must increase nucleotide production before or during $\mathrm{S}$ phase to meet the needs of protein expression and DNA replication. IMPDH cytoophidia can also be induced in cells by the addition of inhibitors that impede GTP biosynthesis, such as MPA (Ji et al. 2006), ribavirin (Carcamo et al. 2014), or by depleting essential purine precursors (Calise et al. 2014). Disassembly of cytoophidia can be induced by the addition of the downstream product guanosine or GTP (Ji et al. 2006; Carcamo et al. 2011; Thomas et al. 2012). Cellular IMPDH cytoophidia can also be reduced by disrupting the cell cycle through the PI3K-AKT-mTOR pathway using a PI3K inhibitor (Chang et al. 2015). These results again support the hypothesis that upregulation of purine nucleotide synthesis promotes IMPDH cytoophidia assembly. IMPDH form abundant cytoophidia in mouse pancreatic islet cells, which correlate with insulin secretion (occurring in the fed, but not starved state) (Chang et al. 2015), and they are also developmentally regulated (Aughey et al. 2014). In summary, cytoophidia by IMPDH1 and IMPDH2 appear to be required for producing sufficient amounts of guanine nucleotides to maintain normal cell proliferation when the intracellular IMPDH level is not adequate (Keppeke et al. 2018).

Cellular cytoophidia are large structures, which are likely composed of bundles of filaments. Human IMPDH2 filaments have been structurally characterized by electron microscopy (Labesse et al. 2013; Anthony et al. 2017) (Fig. 5c, d). EM revealed two types of filament structures, one appearing under conditions giving rise to activated IMPDH (the octamers have an "open" configuration and they form with $\mathrm{NAD}^{+}$), and the other with inactive (the octamers have a "closed" conformation and form with GTP)(Anthony et al. 2017) (Fig. 5d). Both types of filaments are induced by ATP and are composed of stacked octamers with $30^{\circ}$ (open conformation) or $35.5^{\circ}$ (closed conformation) between them (Anthony et al. 2017) (Fig. 5d-e). A domain of IMPDH, the CB domain, binds $\mathrm{MgATP}$ and is responsible for allosteric activation of Pseudomonas aeruginosa IMPDH (Labesse et al. 2013), and human IMPDH without CB domain do not form cytoophidia (Keppeke et al. 2018).

Although the cellular superstructure known as ctyoophidia (or "rods and rings") appear to form under conditions of active IMPDH, the EM study finds that filaments may be composed of either active or alternatively of inactive enzymes. To investigate the relationship between enzyme activity and filamentation, a study was performed with mutations that either impair filamentation or result in constitutive formation of filaments (Anthony et al. 2017). It was found that wild-type IMPDH and mutants that either promote or inhibit polymerization have comparable catalytic activity, substrate affinity, and GTP sensitivity. This is also true when expressed in living cells (HEK 293) (Anthony et al. 2017). Unlike other metabolic filaments (i.e., CTPS), which selectively stabilize active or inactive conformations, recombinant IMPDH filaments accommodate multiple states. These conformational states are finely tuned by substrate availability and purine balance (Anthony et al. 2017). It has been suggested that polymerization may allow cooperative transitions between states, where effectors can rapidly modulate the activity of the entire IMPDH population perhaps using a switch-like transition (Anthony et al. 2017). They also postulate that the filaments could also be a platform for other regulatory proteins, the conformational state providing a physical signal for the level of guanine biosynthetic activity (Anthony et al. 2017). Finally, given the transcriptional activity observed in Drosophila IMPDH, the filaments could serve to sequester IMPDH from its nuclear functions (Kozhevnikova et al. 2012; Anthony et al. 2017).

\section{Glutamine synthetase}

Glutamine synthetase (Gln1) is an essential enzyme that synthesizes glutamine from glutamate and ammonium in an ATPdependent manner (Fig. 6a). It is found in all forms of life, and for plants, it is a critical factor in nitrogen fixation. In bacteria, it functions as a regulator of glutamine level. In higher organisms, it has critical functions in maintaining proper hormonal levels in neurons, particularly astrocytes. The enzyme catalyzes the activation of glutamate to phosphoglutamate with energy from ATP. Upon activation with ATP, the structure of the active site around the phosphoglutamate undergoes a rearrangement allowing binding of ammonium (Eisenberg et al. 2000). After enzymatic ammonium deprotonation, the ammonia attacks the intermediate, yielding products which may leave by opposite sides of the enzyme ring structure (Eisenberg et al. 2000). 
Fig. 6 Reactions catalyzed and structures formed by glutamine synthetase. a Reaction catalyzed by glutamine synthetase (EC 3.5.1.2). b mCherry labeled glutamine synthetase forms cytoophidia in yeast cells under nutrient starvation (adapted with permission from Petrovska et al. 2014). c Asymmetric unit of $x$-ray crystal structure (PDB code 3FKY (He et al. 2009b)) showing pentameric rings that stack headto-tail to form a decamer, that further stacks with another decamer in the head-to-head fashion. d Electron microscopy of glutamine synthetase cytoophidia in yeast cells showing stacked filamentous fine structure (adapted with permission from Petrovska et al. 2014). e Negative stain EM of the constitutive cytoophidia forming mutant R23E of yeast glutamine synthetase showing filaments that appear to be composed of stacked rings (adapted with permission from Petrovska et al. 2014)
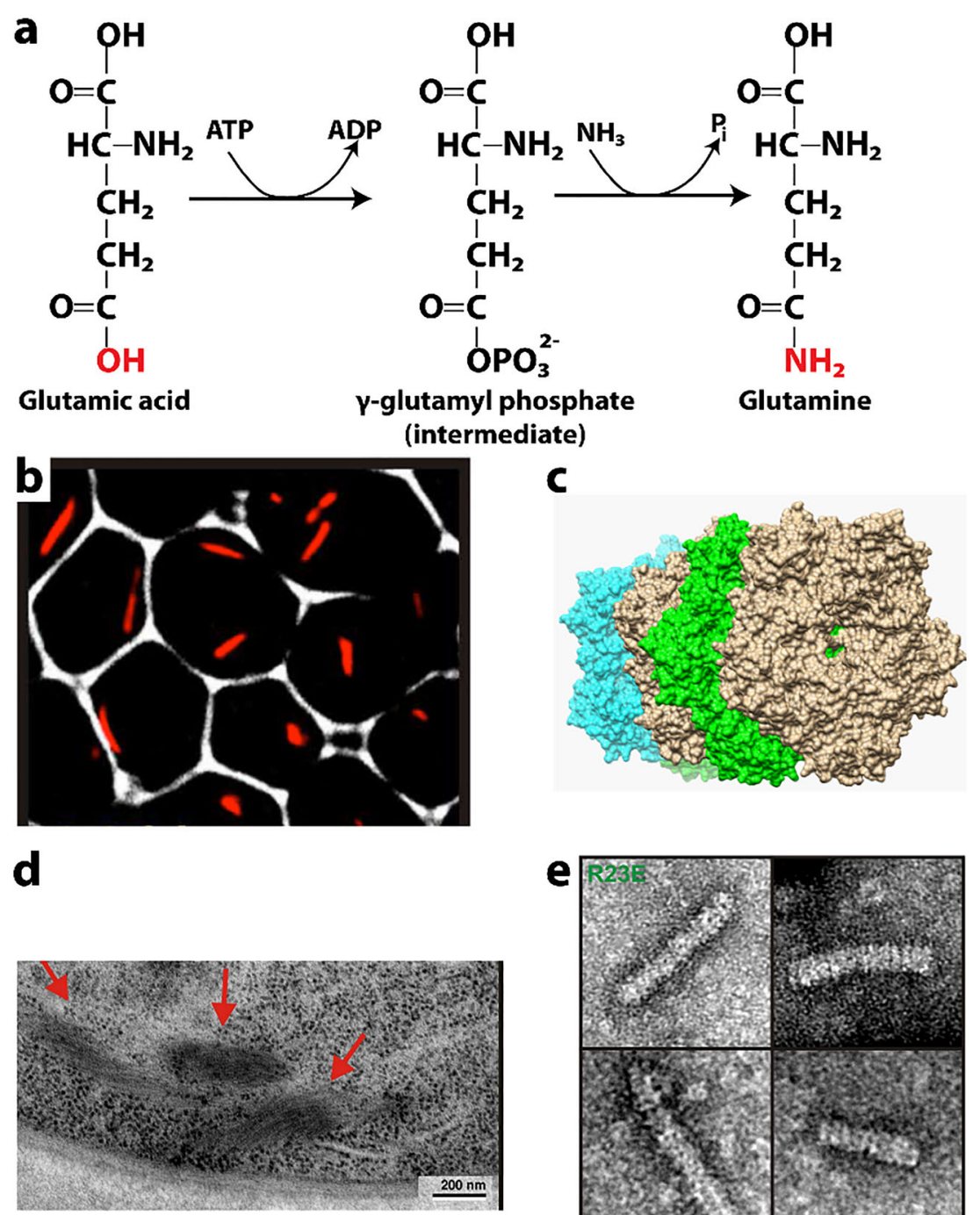

The various isoforms of glutamine synthetase are regulated in many ways (Eisenberg et al. 2000). Many free amino acids inhibit the enzyme by binding the substrate site. Glycine, alanine, and serine have backbones that bind in the same orientation as the substrate glutamate. The activating nucleotide site can be inhibited by ADP, AMP, and GDP. In prokaryotes, glutamine synthetase is regulated by adenylylating a critical active site tyrosine. However, all 12 protomers of the dodecamer need to be modified in order to stop the enzyme's activity. This can be an energy intensive process because the adenylylation requires ATP. In E. coli, it is known that regulation is also carried out by specific proteins at the genetic level. Presumably, genetic regulation is found in all branches of life. In eukaryotic non-brain tissue, GS is inhibited by glutamine metabolism end products. However, brain tissue does not seem inhibited this way. Filamentation may be another form of controlling activity, likely by inhibition, at the structural level and we are just beginning to investigate this.
Gln1 is an isoform of glutamine synthetase that was found in metabolic screens of yeast cells under starvation as well as heat stress conditions where fluorescent versions of the enzyme were seen microscopically in foci, rods, rings, fibrils, and related large self-assemblies (often referred to as cytoophidia) (O'Connell et al. 2014). The effect of various fluorescent tags on Gln1 in yeast was examined, and the cytoophidia were shown to persist under starvation conditions when Gln1 was fused to mCherry, and tetracysteine-binding fluorophores (Petrovska et al. 2014) (Fig. 6b). The conclusion of Petrovska and coworkers is that Gln 1 in yeast is a cytoophidia-forming enzyme that conserves energy involved in protein synthesis by storing this important metabolic catalyst until starvation conditions are reversed (Petrovska et al. 2014). Interestingly, the cytoophidia formation started nearly one hour (50 $\mathrm{min}$ ) after advanced starvation conditions. Upon reintroduction of glucose, cytoophidia dissolved within $18 \mathrm{~min}$ and cells were available to reenter the cell cycle. 
Glutamine synthetase cytoophidia have been investigated by two groups (Narayanaswamy et al. 2009; Petrovska et al. 2014). The fluorescently labeled structures in yeast resemble punctate foci or short rods. Foci formation by Gln1 was shown to be stimulated by the absence of adenine in growth media, and could be reversed upon the addition of glucose (Narayanaswamy et al. 2009). Gln1 punctate body formation was not inhibited by the addition of the protein translation inhibitor cycloheximide, while those of Gln1 were not. These foci did not colocalize with P-body marker proteins and only in a limited way with actin bodies. Pelleted purified Gln1 punctate foci could not be dissolved into soluble protein by the addition of rich media.

The crystal structure of Gln1 from yeast was solved and found pentameric rings that stacked in the asymmetric unit (a total of 4 rings) (Fig. 6d) (He et al. 2009b). This suggests that stacking of the rings is the basis for forming the larger selfassemblies visible in cells and by EM. There is an asymmetry to the stacking. The decamer forms from two pentameric rings that sit next to each other (i.e., front-to-back) with a $30^{\circ}$ rotation between the rings. The interaction between pentamers in the functional decamer seems to be mediated by a large amount of looping structures and driven primarily by hydrophobic interactions $\left(1900 \AA^{2}\right)$ and 10 hydrogen bonds. The back-to-back interaction $\left(1700 \AA^{2}\right)$ that allows decamers to self-associate into the stack of 4 pentameric rings seen in the asymmetric unit has a $10^{\circ}$ rotation in the same direction as that between rings in decamers. The residues in the area defining the decamer-decamer interaction seem to be highly charged which seems to mediate binding presumably in $\mathrm{pH}$ or chargedependent manner.

Petrovska and coworkers also formed an inducible Gln1 mutant yeast strain that could be controlled (Petrovska et al. 2014). By stopping further translation of Gln1 under starvation conditions, they found that the yeast was able to respond to new supplies of glucose. This indicates that the formed cytoophidia from Gln1 could form the basis of new active enzymes once the cytoophidia dissolved. They also used correlative light electron microscopy to show the foci and punctae seen in fluorescent images are in fact clusters of aligned fiber segments approximately 1 micron long (Fig. 6e) (Petrovska et al. 2014). Further, attempts to purify the fibers from mutants or starved cells, resulted in dispersions of decamers. However, fibers could be reconstituted in vitro by addition of $200 \mathrm{mg} / \mathrm{mL}$ Ficoll 70 as a crowding agent.

Enzymatic activity falls $60 \%$ for mutants in low $\mathrm{pH}$ media or starved cells relative to well-fed wild type enzyme. This activity can be recovered for the wild type or in some mutants, by reversing cellular or in vitro conditions. The loss in activity seems to correlate with the extent of cytoophidia/fiber formation (Petrovska et al. 2014). Gln1 has a theoretical pI of about 6. Changes in cytosolic $\mathrm{pH}$ would then reduce its net charge indicating a possible electrostatically governed interaction.
Alternatively, protons could act as allosteric effectors changing the structure and/or interfaces. Charge flipping by the mutation E186K was seen to be important in keeping pentameric rings dispersed (Petrovska et al. 2014). But then charge reversal for R23E stabilizes cytoophidia and filaments made of the same rings (Petrovska et al. 2014). Thus, there appears to be an overall electrostatic effect, for example, increasing the negative charge stabilizes filaments, while increasing the positive charge stabilizes pentameric rings.

Petrovska and coworkers tested the hypothesis that these residues are responsible for cytoophidia formation in a $\mathrm{pH}$ dependent manner (Petrovska et al. 2014). Interestingly, as the cells become acidic due to starvation conditions (Orij et al. 2009; Dechant et al. 2010; Orij et al. 2011), the cytoophidia formation seems to be enhanced. In glucose containing media with well-fed yeast, the $\mathrm{pH}$ was dropped by addition of the chemical reagent 2,4-dinitrophenol and cytoophidia were observed. Mutants tested were in several classes. E186K, P83R, and T49E were capable of preventing cytoophidia formation. Y81A had enhanced starvation-induced cytoophidia and R23E formed cytoophidia in both starvation and well-fed conditions. In fact, they found that the Y181A mutant had decreased rate of return to normal cell cycle after starvation induced cytoophidia. They concluded that the return to normalcy is limited by the rate of fiber dissolution. These cytoophidia-stabilizing mutants grew poorly in regular media but could grow normally when the media was supplemented with glutamine. Interestingly, the mixed effect T49E, R23E double mutant shows a strong resistance to cytoophidia formation indicating the relative strength of these two mutants. Imaging of the self-assemblies of purified R23E Gln1 from yeast (a mutant form that constitutively formed cytoophidia in cells (Petrovska et al. 2014)) using negative stain EM shows filaments that appeared to be composed of stacked rings, as was predicted by the x-ray crystal structure (Fig. 6c).

There are a number of advantages thought to be important for Gln1 self-association. There is likely a resistance to bulk autophagy for these enzymes. Also, having functional, oligomerized enzymes ready to react as cellular conditions revert from starvation is an advantage over having to synthesize new enzymes from nucleic acid sequences. This further conserves cellular energy as has been proposed for mammalian cells containing $\mathrm{ADF} /$ cofilin filaments (Bernstein et al. 2006; Bernstein and Bamburg 2010). In fact, it may be that there is a small amount of inactive cytoophidia that provides buffering from metabolic fluctuations (enzyme inactivation by filament formation may provide a buffer against metabolic fluctuations, thus preventing accidental re-entry into the cell cycle).

Activity in the fibrillar (cytoophidic) state seems to be reduced. It is not clear if this is due to blocking of the active site or constraint of conformational changes necessary for activity or if there's simply a charge interaction within the active site 
itself. Fibrillar disassembly presumably happens as rings dissociate. Microscope images of the fibers are seen to "disappear" on the micron length scale with the reestablishment of well-fed conditions or increased pH (Petrovska et al. 2014). This suggests that the unbinding of fibers from each other as well as disassembly of the stacked ring filament structure happens in concert.

Gln1 cytoophidia in yeast, then, may be a storage depot for enzymes. This is seen in other enzymes like Ura7/Ura8, fibers of Gcd2, Gcd6, Gcd7, Gcn3, and Sui2 (Noree et al. 2010). Other researchers have noticed that quiescence-associated subcellular structures, such as proteasome storage granules and actin bodies, also form in a pH-dependent manner and has concluded that the drop in intracellular $\mathrm{pH}$ is important for their formation (Peters et al. 2013). The establishment of $\mathrm{pH}$ as an important messenger is furthered by work of Isom and coworkers who show that the $\mathrm{G} \alpha$ subunit of $\mathrm{G}$-proteins acts as a pH sensor (Isom et al. 2013). In these systems, signaling becomes more quiescent as the $\mathrm{pH}$ is dropped. This strongly reinforces the notion that intracellular $\mathrm{pH}$ changes serve as a global messenger to signal the depletion of energy during starvation and cellular quiescence (Dechant et al. 2010; Orij et al. 2012).

\section{$\beta$-Glucosidase}

A plastidal $\beta$-glucosidase from Avena sativa (oat) is activated during fungal infections. Antifungal precursors are stored in plant vacuoles and the enzyme activates these avenacosides by hydrolysis to form antifungal 26-desglucoavenacosides. The enzyme is a globular $\alpha / \beta$ barrel with two catalytic glutamic acid residues that act as nucleophiles or as an acid/ base catalyst. $\beta$-Glucosidase from oat (Avena sativa) hydrolyzes the $\beta$-glucoside avenacoside to C26-desglucoavenacosides. This enzyme is nominally a homohexamer that hydrolyzes $\beta 1-4$ glucose bonds; it also cleaves avenacosides as an antifungal defense. Antifungal precursors are stored in plant vacuoles and the enzyme activates these avenacosides by hydrolysis to form antifungal 26-desglucoavenacosides.

It has been known since 1965 that the oat $\beta$-glucosidase forms in vivo fibrils named "stromacenters" (Gunning 1965). There are two isomers of oat $\beta$-glucosidase, As-Glu- 1 and AsGlu-2. As-Glu-1 is sufficient to form filaments but can also induce fibrillar or filamentous formation with As-Glu-2 which normally forms a homodimer (Kim et al. 2000). This filament resists $\mathrm{pH}$ and non-ionic detergents but can be disassembled with $\mathrm{CaCl}_{2}$ (Kim et al. 2005). Interestingly, Hevea $\beta$ glucosidase also forms long filamentous assemblies, but the enzyme in this species has not been studied extensively by higher resolution techniques or kinetic modeling (Gunning 1965; Nisius and Ruppel 1987).

Finite sized oligomers of oat $\beta$-glucosidase, trimers, hexamers, and multimers, are seen by EM (Kim et al. 2000;
Kim et al. 2005). The protein is seen as a trimeric ring that dimerizes along the ring's axis into hexamers. Larger ordered oligomers also stack along the axis of the ring into a filament (Fig. 7). Through detailed reconstructions there seems to be a difference in the twist between neighboring trimers forming the "tunnel" housing the active sites. In the multimers, the twist between trimers is $38^{\circ}$; however, in the non-filamented hexamers, this is $60^{\circ}$. The smaller twist seems to result in smaller side fenestrations presumably changing access to the active site. This may explain both the reduced $\mathrm{K}_{\mathrm{M}}$ and $\mathrm{k}_{\mathrm{cat}}$, since a caging effect may increase binding affinity to both substrates and products (Kim et al. 2005). In addition, the $\mathrm{k}_{\mathrm{cat}} / \mathrm{K}_{\mathrm{M}}$ of the multimers (i.e., filament) is twice that of the hexamer (Kim et al. 2005). Hence, the caging effect may result in increased substrate discrimination, such as in preference for avenacoside substrates over other kinds of $\beta$-glucosides. Alternatively, or in addition, the physiological role of filamentation may be to create a strongly localized concentration gradient of antifungal product near the formation of the fibers of assembled filaments.

\section{Hydrogen-dependent $\mathrm{CO}_{2}$ reductase}

The interconversion of $\mathrm{CO}_{2}$ and formic acid is an important reaction in bacteria, and enzymes catalyzing the oxidation of formic acid to $\mathrm{CO}_{2}$ and concomitant reduction of protons to molecular hydrogen have been known for decades (Sawers 1994; Maia et al. 2015). However, only relatively recent has an enzyme capable of the reverse been characterized. This enzyme utilizes molecular hydrogen as electron donor for the reversible reduction of $\mathrm{CO}_{2}$ (the first reaction of the acetyl-CoA or Wood-Ljungdahl pathway) and has been named the hydrogen-dependent $\mathrm{CO}_{2}$ reductase (HDCR) (Fig. 8a). It derives from an acetogenic bacterium, Acetobacterium woodii (Schuchmann and Muller 2013). This pathway is utilized for carbon fixation as well as energy conservation (Poehlein et al. 2012; Schuchmann and Muller 2013; Schuchmann and Muller 2014). Reduction of $\mathrm{CO}_{2}$ is also of interest in biotechnological applications such as removal of $\mathrm{CO}_{2}$ from the atmosphere, hydrogen storage, and hydrogen production.

HDCR is composed of four subunits in equal stoichiometry and forms filaments with increased enzymatic activity at concentrations of $0.05 \mathrm{mg} / \mathrm{ml}$ and higher, and in the presence of divalent cations (Schuchmann et al. 2016). The lengths of the filaments are varied and can be more than 0.1 micron in length but with apparent bending indicating flexibility (Fig. 8b) (Schuchmann et al. 2016). Their formation is reversible and dependent on only the presence of divalent cations (e.g., $\mathrm{Mg}^{2+}, \mathrm{Mn}^{2+}$, and $\mathrm{Ca}^{2+}$ ) at concentrations of $2-20 \mathrm{mM}$ (Schuchmann et al. 2016). The width of the filaments is 10 $15 \mathrm{~nm}$, with an apparent helical structure (Schuchmann et al. 2016); however, no three-dimensional analyses or higher 
Fig. 7 Reaction catalyzed and filamentous structures formed by oat $\beta$-glucosidase. a Reaction catalyzed by oat $\beta$-glucosidase (EC 3.2.1.21). b Negative stain EM micrograph of filamentous oat $\beta$-glucosidase (adapted with permission from Kim et al. 2005). c Single particle reconstruction using cryo-EM images of oat $\beta$ glucosidase showing the filamentous form which forms from stacks of hexamers (themselves made of stacked trimeric rings). Scale bars are $20 \mathrm{~nm}$ (adapted with permission from Kim et al. 2005). Left, top view, middle and right, side views. d Hexameric unit from filamentous form shown in c, with additional symmetry applied during refinement. Scale bars are $2 \mathrm{~nm}$ (adapted with permission from Kim et al. 2005). Left, top view, middle and right, side views
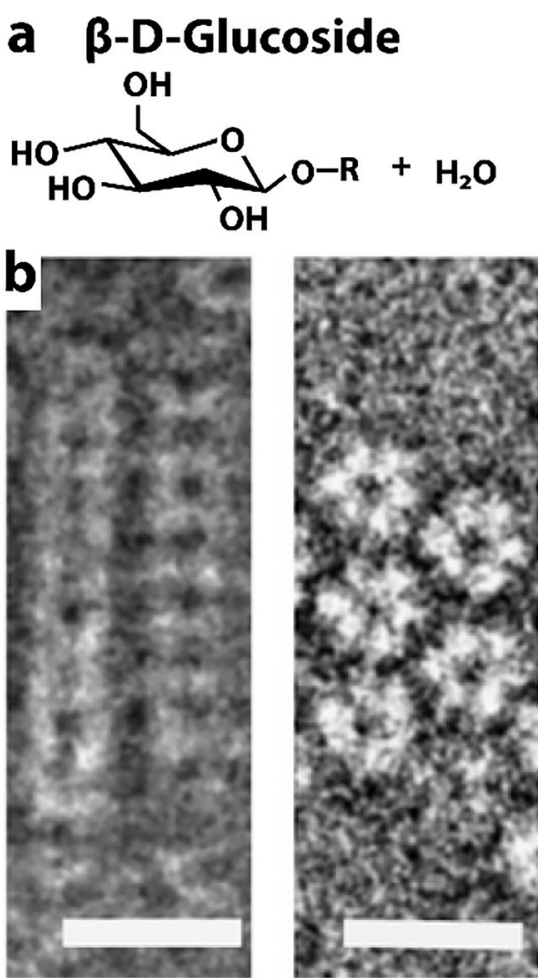
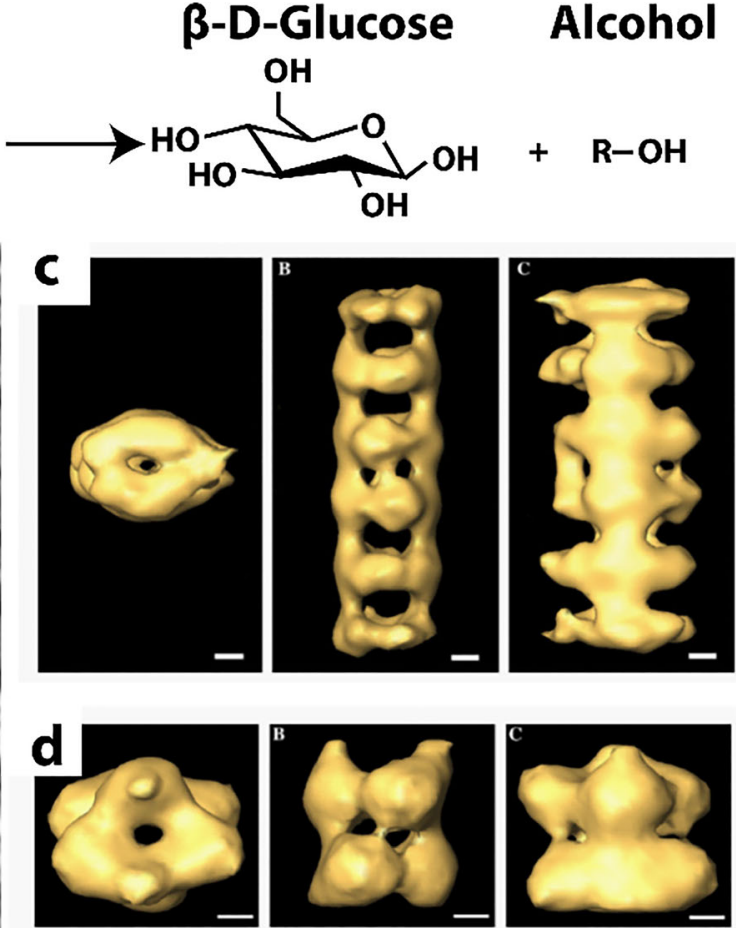

resolution structures of the filament or enzyme protomers have been reported.

Light scattering studies showed that the formation of polymers/filaments follows a first-order process complete after $500 \mathrm{~s}$, but with a fast initial jump (Schuchmann et al. 2016). Enzyme activity assays indicate that the polymeric form is more active by approximately 2 -fold than the nonpolymerized form (Schuchmann et al. 2016). Hence, authors speculate that filamentation may be a regulatory mechanism to activate HDCR. Alternatively, it could stabilize the enzyme, regulate its degradation, or act as a scaffold for other enzymes, making use of close proximity for electron tunneling between iron-sulfur clusters of adjacent enzymes. Finally, it may serve to protect the iron-sulfur clusters from oxidative damage (Schuchmann et al. 2016).

\section{Nitrilase}

Nitrilases are a family of enzymes that convert nitriles to the corresponding acid and ammonia (Fig. 9a), and occasionally

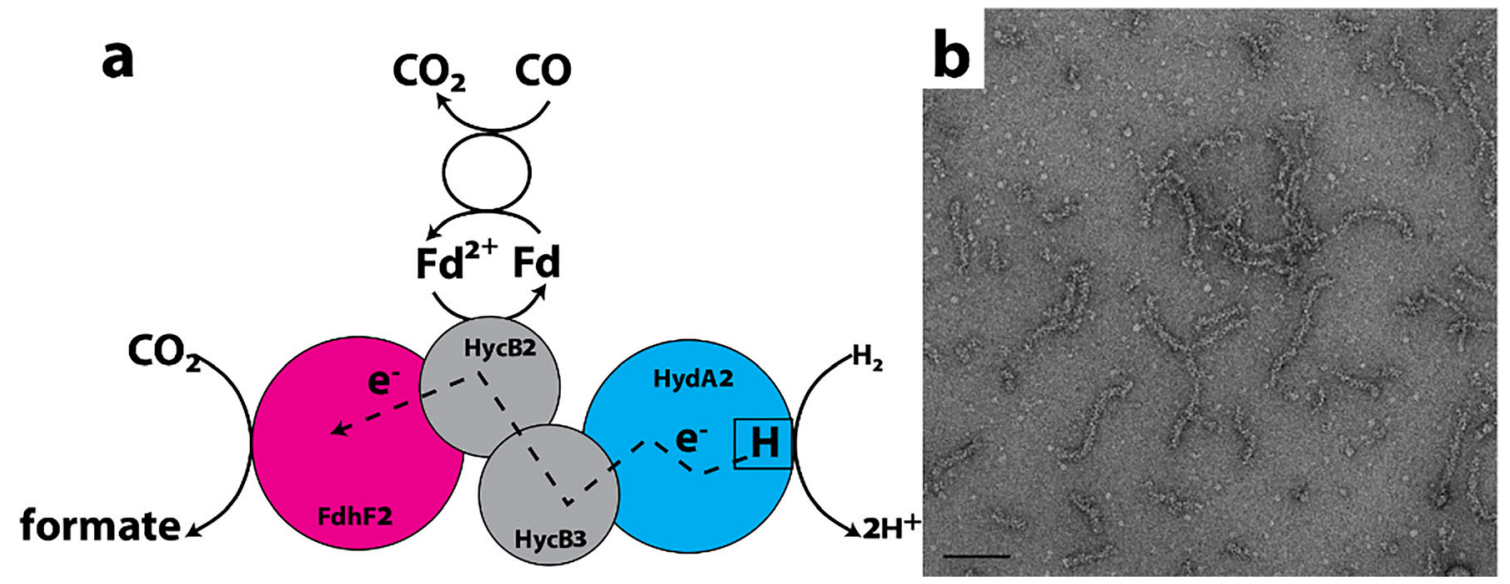

Fig. 8 Reaction catalyzed and assemblies formed by A. woodii $\mathrm{CO}_{2}$ reductase. a Enzyme reaction carried out by hydrogen-dependent $\mathrm{CO}_{2}$ reductase (HDCR) from $A$. woodii. Electrons for $\mathrm{CO}_{2}$ reduction are either provided by the hydrogenase subunit HydA2, where hydrogen oxidation takes place, or by reduced ferredoxin $(\mathrm{Fd})$. Electrons are delivered to the active site for $\mathrm{CO}_{2}$ reduction in $\mathrm{FdhF} 2$ via the electron-transferring subunits $\mathrm{HycB} 2 / 3$. Fdh, formate dehydrogenase; Hyd, hydrogenase; $\mathrm{CODH}, \mathrm{CO}$ dehydrogenase (adapted with permission from Schuchmann and Muller 2013). b Negative stain EM micrograph of $\mathrm{CO}_{2}$ reductase showing filamentous assemblies (adapted with permission from Schuchmann et al. 2016). Scale bar represents $100 \mathrm{~nm}$ 
release an amide product (Pace and Brenner 2001; Fernandes et al. 2006). The majority of known enzymes are from bacteria, fungi, and plants. The enzyme composition is homodimeric with a $\alpha \beta \beta \alpha-\alpha \beta \beta \alpha$ sandwich fold and an active site containing four positionally conserved residues: Cys, Glu, Glu, and Lys. Nitrilases are attractive biocatalysts for the production of fine chemicals and pharmaceutical intermediates and also are used in the treatment of toxic industrial effluent and cyanide remediation. Nitrilases have been visualized as dimers, tetramers, hexamers, octamers, tetradecamers, octadecamers, and variable length helices, all with the same basic dimer interface, and were known since 1977 to form large aggregates upon activation (Harper 1977b; Harper 1977a). Self-assemblies are also visible in cells. GFP labeled Nitrilase I in Arabidopsis was found to form "aggregates" in cells following wounding and just prior to cell death (Fig. 9b) (Cutler and Somerville 2005). One function of nitrilases in vivo may be in detoxification, hence why these self-assemblies (which likely contain activated nitrilases) form upon wounding with herbicides. Negative stain electron microscopy shows the fine structure of activated, self-associated nitrilase enzymes as short spirals consisting of 8 to 22 subunits or long filaments (Fig. 9c) (Sewell et al. 2003; Thuku et al. 2007; Vejvoda et al. 2008; Woodward et al. 2008; Dent et al. 2009). These show a left-handed spiral with about 4-5 copies of the dimer per turn (Fig. 9d-f). The activity of a nitrilase was shown to increase with formation of large aggregates from inactive homodimers
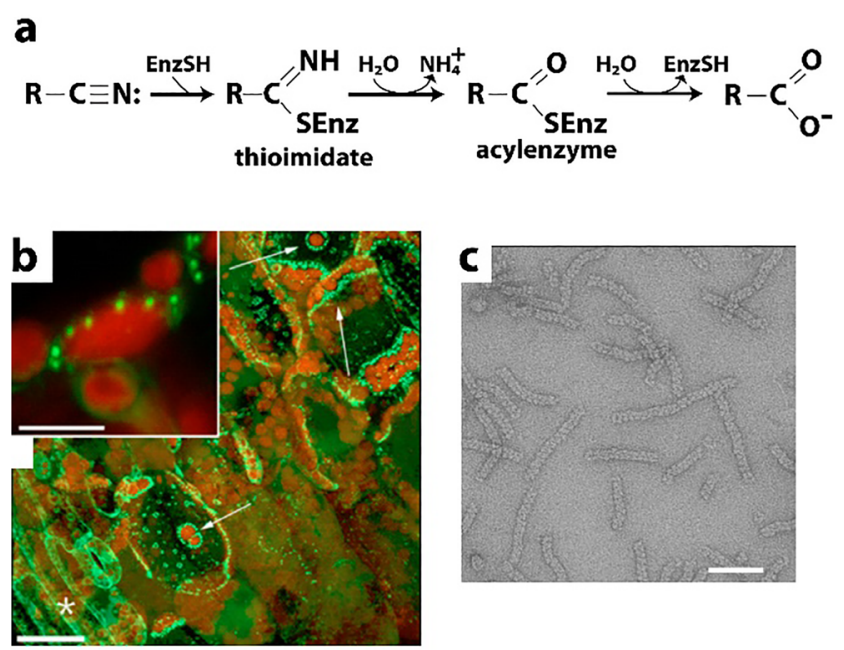

Fig. 9 Reactions catalyzed and structures formed by nitrilases. a An example of one of the many reactions carried out by nitrilase superfamily members (EC 3.5.5.1) (adapted with permission from Pace and Brenner 2001). b In a plant cell, GFP labeled Nitrilase forms selfassemblies (green) around chloroplasts (red) following cell injury. Scale bars represent 10 microns (top) and 25 microns (bottom) (adapted with permission from Cutler and Somerville 2005). c Negative stain EM micrograph of Capsella rubella nitrilase 2 filaments formed in vitro. Scale (presumably the helical filaments) (Nagasawa et al. 2000). This aggregation could be induced by addition of benzonitrile, as well as the presence of salts and organic solvents (the best conditions were 10\% saturated ammonium sulfate and $50 \%(\mathrm{v} / \mathrm{v})$ glycerol, and by preincubation at increased temperatures or enzyme concentrations). Helical filament were shown to form by truncation of the Cterminal 39 amino acid residues, perhaps indicating their involvement in regulating activation of the enzyme via helical filament formation (Thuku et al. 2007). Interestingly, the substrate specificity of plant nitrilase complexes was shown to be related to the helical twist of its filamentous state (Woodward et al. 2018). Previously, attempts to swap specificities between nitrilase enzymes by swapping active site residues failed. However, helical twist and substrate size were found to be correlated among different nitrilase enzymes, and when binding pocket residues are exchanged between two nitrilases that show the same twist but different specificities, their specificities could be swapped. Modifying nitrilase helical twist, by either exchanging an interface residue or by imposing a different twist without altering any binding pocket residues, changes substrate preference. This is significant, as it shows that one function of filament formation is in determining substrate specificity among evolutionarily related enzymes (Fig. 9g). In addition, Thuku and coworkers speculate that the helix could form docking sites for other associated enzymes and that the central hole channels substrates and products (Thuku et al. 2009).
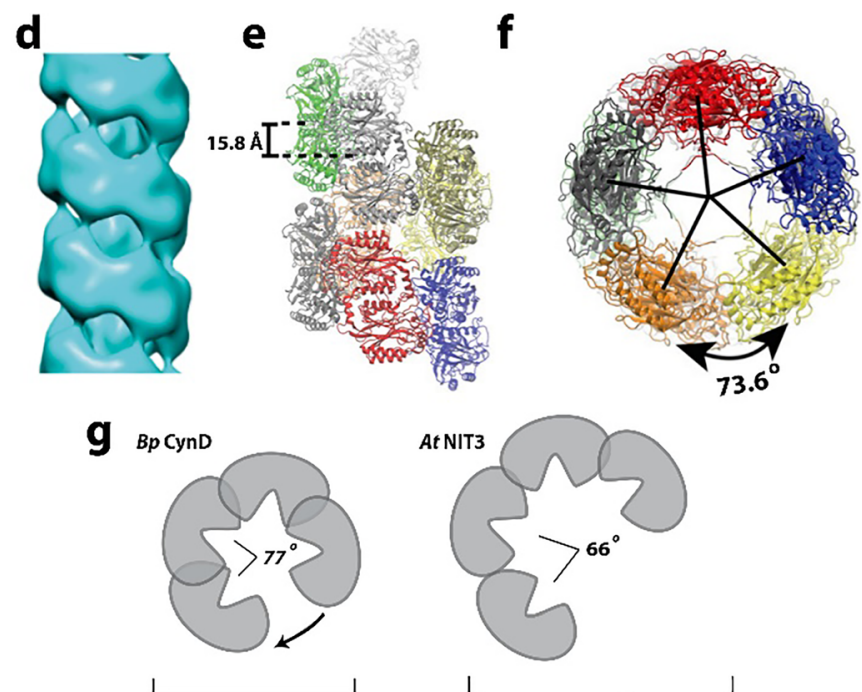

bar represents $50 \mathrm{~nm}$ (adapted with permission from Woodward et al. 2018). d Single particle reconstruction of Capsella rubella nitrilase 2 from negative stain EM (adapted with permission from Woodward et al. 2018). e-f Orthogonal views of model built from flexible fitting of the Rhodococcus rhodochrous nitrilase structure into the EM reconstruction (adapted with permission from Chan et al. 2011). g Cartoons of two nitrilase structures (Bacillus pumilus $\mathrm{CynD}$ and Arabidopsis thaliana nitrilase 3) (adapted with permission from Woodward et al. 2018) 


\section{CoA-dependent acetaldehyde and alcohol dehydrogenase (AdhE)}

The AdhE enzyme is a type of enzyme found only in bacteria and photosynthesizing unicellular organisms. AdhE catalyzes the conversion of an acyl-coenzyme A to an alcohol via an aldehyde intermediate, which is coupled to the oxidation of two NADH molecules to maintain the $\mathrm{NAD}^{+}$pool during fermentative metabolism (Fig. 10a). AdhE from Escherichia coli is a homopolymer of $96-\mathrm{kDa}$ subunits harboring three $\mathrm{Fe}^{2+}$-dependent catalytic functions: acetaldehyde-CoA dehydrogenase, alcohol dehydrogenase, and pyruvate formatelyase (PFL) deactivase. AdhE forms helical structures in cells (as well as in vitro) called "spiralosomes." Spiralosomes were first identified by 1975 in Lactobacillus fermti F-4 (Kawata et al. 1975), but have since been identified in several bacterial species since (see Laurenceau et al. 2015) (Fig. 10b). Having confirmed the identity of spiralosomes as composed of the enzyme AdhE, Kessler and coworkers describe their structure as a left-handed helical assembly of 20-60 subunits in rods of 45-120 nm in length (Kessler et al. 1992) (see also Fig. $10 \mathrm{c}, \mathrm{d})$. They also show that subunit packing is widened along the helix axis when $\mathrm{Fe}^{2+}$ and $\mathrm{NAD}^{+}$are present, which is accompanied by a change in width and pitch consistent with opening and stretching of the helix. Extance and coworkers purified recombinant Geobacillus AdhE and found it to form large assemblies refractory to crystallization (Extance et al. 2013). Crystallization of the ADH domain however was successful. By combining this structure with a homology model of AldDH, they were able to propose a molecular model for the AdhE spiralosome (Fig. 10e). This model has 7 copies per turn with right-handed helical geometry. Though it is not known what effect on enzyme activity this structure has, or if its structure is modulated in the cell, Extance and coworkers

a
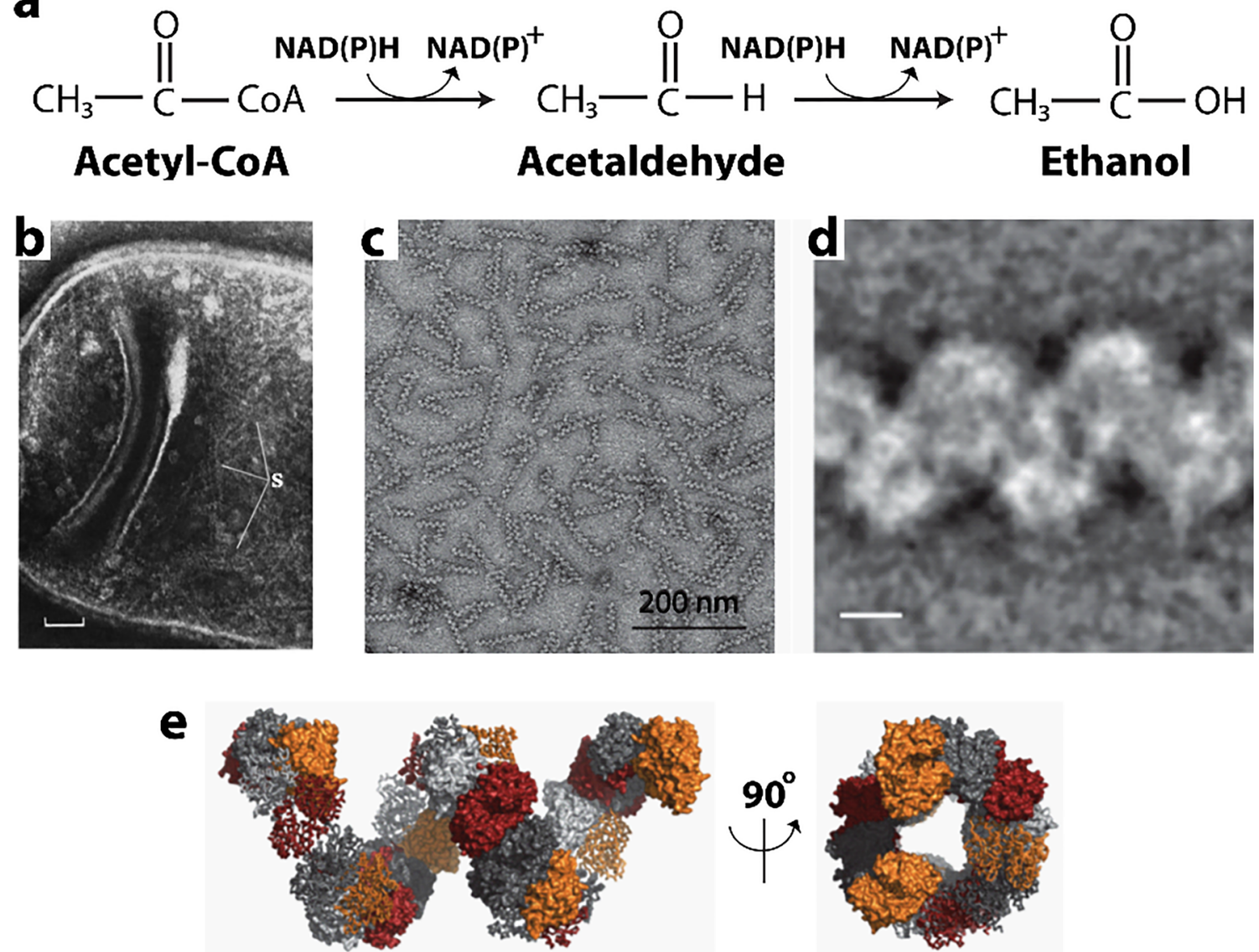

Fig. 10 Reactions catalyzed and structures formed by AdhE. a An example reaction carried out by $\operatorname{AdhE}(E C: 1.2 .1 .10)$. This enzyme can follow the same pathway with longer aliphatic chains including proprionyl-CoA, valeryl-CoA, butyryl-CoA, and hexanoyl-CoA. b Formation of helical structures in cells viewed using negative stain EM. Portion of a partially lysed cell found in the cytoplasmic membrane fraction obtained from $L$. casei Var. casei 1001 . Fine spirals (S) are seen in a cluster within the degraded cytoplasm. Scale bar is $100 \mathrm{~nm}$ (adapted with permission from Kawata et al. 1975). c Negative stain EM micrographs of immunopurified Streptococcus pneumoniae "spiralosomes," helical structures formed by AdhE (adapted with permission from Laurenceau et al. 2015). d A representative class average of the $S$. pneumoniae spiralosome. Scale bar is $5 \mathrm{~nm}$ (adapted with permission from Laurenceau et al. 2015). e Two views of a spiralosome model built with the x-ray crystal structure of $G$. thermoglucosidasius spirosome (Extance et al. 2013). The color of the individual protomers alternate between grayscale and domain-coded color representation, and successive AdhE dimers alternate between ribbon and surface representation (adapted with permission from Laurenceau et al. 2015) 
propose that it may enhance catalytic efficiency through substrate channeling of the reactive acetaldehyde intermediate, or alternatively, provide some stabilization of the protein (Extance et al. 2013). Kessler and coworkers hypothesize that the two forms visualized in their study represent the inactive, closed helical form, and the open, active form (Kessler et al. 1992).

\section{Glutamate dehydrogenase}

Glutamate dehydrogenase (GDH, EC 1.4.1.2) is found in all living organisms and catalyzes the reversible oxidative deamination of L-glutamate to 2-oxoglutarate using $\mathrm{NAD}(\mathrm{P})^{+}$as a coenzyme (Hudson and Daniel 1993) (Fig. 11a). In bacteria, the ammonia produced by GDH is assimilated to amino acids via glutamate and aminotransferases (Lightfoot et al. 1988). In plants, the enzyme can work in either direction depending on environment and stress (Mungur et al. 2005; Grabowska et al. 2011). Transgenic plants expressing microbial GDHs are improved in tolerance to herbicide, water deficit, and pathogen infections (Lightfoot et al. 2007). GDH from animals, but not other kingdoms, is allosterically regulated by a wide array of ligands (Frieden 1959a; Yielding and Tomkins 1961; Tomkins et al. 1962; Frieden 1965; Sener and Malaisse 1980; Dieter et al. 1981; Bailey et al. 1982). Mammals encode two important GDHs, GLUD1 and GLUD2. GLUD1 is a mitochondrial matrix enzyme and plays a key role in nitrogen and glutamate metabolism and energy homeostasis. It is allosterically activated by ADP and inhibited by GTP and ATP. This dehydrogenase is expressed at high levels in liver, brain, pancreas, and kidney, but not in muscle. In the pancreatic cells, GLUD1 is thought to be involved in insulin secretion mechanisms. In nervous tissue, where glutamate is present in concentrations higher than in the other tissues, GLUD1 appears to function in both the synthesis and the catabolism of glutamate and perhaps in ammonia detoxification. Two clinical conditions are associated with mutations in GLUD1: (1) familial hyperinsulinism, characterized by hypoglycemia that ranges from a severe neonatal-onset that is difficult to manage, to a childhood onset disease with mild symptoms and difficult to diagnose hypoglycemia, and (2) hyperammonemia/hyperinsulinism, associated with mild to moderate hyperammonemia and with relatively mild, late-onset hypoglycemia (Stanley et al. 1998; Stanley et al. 2000). These mutations affect regulation by GTP, causing failure to be inhibited by GTP. Its link to insulin has made it a target for drug discovery for activators to increase insulin secretion in patients with diabetes ( $\mathrm{Li}$ et al. 2011, 2012b; Smith and Smith 2016). GLUD2 is localized to the mitochondrion and acts as a homohexamer to recycle glutamate during neurotransmission (Shashidharan and Plaitakis 2014).

Over 60 years ago, GDH purified from bovine liver was shown to form polymers in vitro. Olson and Anfinsen showed in 1952 using sedimentation equilibrium that GDH behaved anomalously, sedimenting at much larger molecular weights than expected (Olson and Anfinsen 1952). Eisenberg and Reisler used light scattering to show evidence of polymerization (Eisenberg and Reisler 1971). Huang and Frieden showed in 1972 how ligands affect polymerization; GTP, a known inhibitor, causes depolymerization, and ADP, a known activator, can mitigate this effect (Huang and Frieden 1972). Josephs and Borisy investigated the structure of assemblies of glutamic dehydrogenase in 1972 and found that they formed linear polymers that further assembled into a helical tube, with
Fig. 11 Reactions catalyzed and structures formed by glutamate dehydrogenase (GDH). a Reaction catalyzed by glutamate dehydrogenase (EC 1.4.1.2). b Helical tubes of bovine GDH in vitro by negative stain EM (adapted with permission from Josephs and Borisy 1972). c GFP labeled GDH form a rod-like structure or "cytoophidia" in cells (yeast) under nutrient starvation conditions (adapted with permission from Shen et al. 2016)
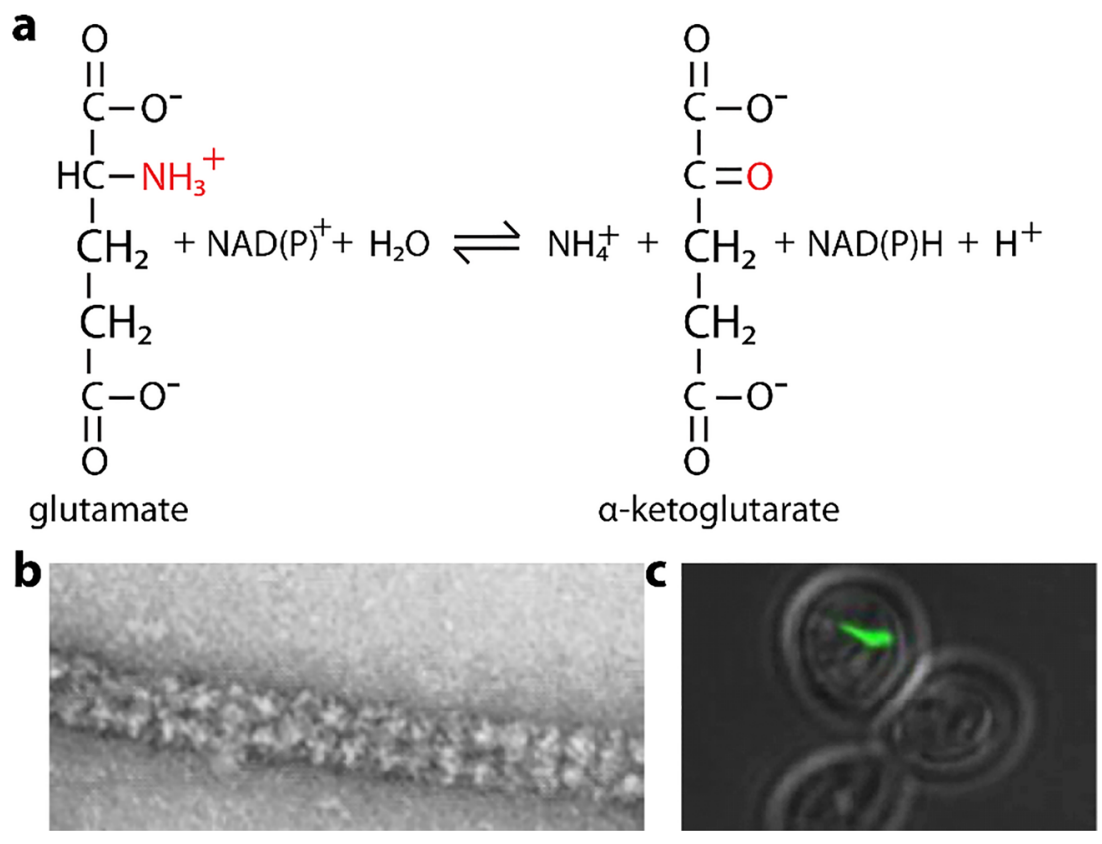
4 linear chains lining the tube (Josephs and Borisy 1972) (Fig. $11 \mathrm{~b})$. Each linear polymer chain was found to be inclined to the tube axis at an angle of $28.5^{\circ} \pm 1.5^{\circ}$, with a pitch of $\sim 800$ $\AA$, with 9-9.3 molecules per turn. However, at least 3 variants of this geometry were also found. Zeiri and Reisler studied the catalytic activity of the polymers in 1978, although the relationship between them remained unclear (Zeiri and Reisler 1978). It is now known that the catalytically active form of GDH is a homohexamer composed of six $54 \mathrm{kD}$ subunits (Frieden 1959b; Cassman and Schachman 1971; Fahien et al. 1989), which can also associate further into filaments (Frieden 1959b; Fahien et al. 1989; Fahien and Macdonald 2011). Binding of leucine, ADP, succinyl-CoA, or BCH to the allosteric sites increases GDH enzyme activity and polymerization of its polypeptide chain, while binding of GTP or palmitoyl-CoA to these sites decreases GDH enzyme activity and causes dissociation of the polypeptide chains from one another (Frieden 1959b; Gylfe 1976; Fahien et al. 1989). The activator and inhibitor sites are overlapping (Frieden 1959b; Fahien et al. 1989), consequently, for example, leucine can displace GTP from the allosteric sites and activate the enzyme (Fahien et al. 1990). Despite all these studies, the role of GDH polymerization on enzyme regulation remains elusive. Finally, Shen and coworkers (re)discovered this phenomenon in 2016 (although on a much larger scale and in vivo) using GFP labeled GDH, which occurred upon nutrient starvation (Fig. 11c), sparking interest in this phenomenon once again (Shen et al. 2016).

\section{Glutaminase}

Glutaminase (EC 3.5.1.2), also known as L-glutamine amido hydrolase, catalyzes the cleavage of glutamine to ammonia and glutamate (Fig. 12a). Olsen and coworkers purified this enzyme in 1970 from pig renal cortex and found it to consist of three forms: T, P, and P-B forms (Olsen et al. 1970). After purification from pig kidney, the enzyme was in the P-B form. It was converted to the $\mathrm{T}$ form by dialysis overnight against $10 \mathrm{mM}$ Tris- $\mathrm{HCl}$ (pH 8.0) containing $3 \mathrm{mM}$ EDTA, and recovered in the supernatant fraction following centrifugation at $80,000 \mathrm{~g}$ for $1 \mathrm{~h}$. The $\mathrm{T}$ form was converted to the $\mathrm{P}$ form by dialysis overnight against phosphate buffer, $50 \mathrm{mM}(\mathrm{pH} 8)$ containing $3 \mathrm{mM}$ EDTA. The P form was further converted to the P-B form by addition of sodium borate, $15 \mathrm{mM}$ ( $\mathrm{pH} 8$ ) to the enzyme in phosphate buffer. The $\mathrm{T}$ form (with a molecular weight estimated at $140 \mathrm{kD}$ by sedimentation velocity, likely a dimer) is non-polymerized and has low to no activity. The P-B form was found to be composed of long, doublestranded left-handed helical structures of various lengths, with a diameter of $\sim 12 \mathrm{~nm}$. The diameter of each of the two strands composing the helix is about $3 \mathrm{~nm}$, and the length of each halfperiod along the helical axis is about $22 \mathrm{~nm}$. Helices with 2-3 turns, as well as $25-30$ turns were observed. The $\mathrm{P}$ form also displayed helical structures in negative stain EM, but were much shorter than the P-B form (produced by adding phosphate-borate buffer. Most were 4-5 turns.

Since this early work many x-ray crystal structures of glutaminase have been revealed. DeLaBarre and coworkers published the first full length structure of human glutaminase in 2011 (DeLaBarre et al. 2011) (Fig. 12b). The structure shows a homotetramer with $\mathrm{C} 4$ symmetry. There is now considerable interest in glutaminase as a drug target to treat cancer because glutamine from blood can be converted to glutamate, which can be oxidized to $\alpha$-ketoglutarate to feed the TCA cycle or be used for the biosynthesis of several other amino acids and lipids (Szeliga and Obara-Michlewska 2009; DeBerardinis and Cheng 2010). In humans, there are two distinct genes encoding glutaminase enzymes: GLS1 and GLS2. The GLS1 enzyme is expressed at high levels in kidney and brain tissue. In the kidney, GLS1 is believed to maintain acid-base balance during metabolic acidosis (Mates et al. 2009; DeBerardinis and Cheng 2010).

GLS2 is predominantly expressed in the liver where it provides nitrogen for the urea cycle. In the central nervous system, GLS1 activity is proposed to generate a significant amount of the total neuronal glutamate pool that in turn acts as an excitatory neurotransmitter (Hamberger et al. 1979; Thanki et al. 1983; Chaudhry et al. 2002). GLS1 activity may therefore be essential for the optimal function of multiple central nervous system glutamate receptors and their downstream roles in both the normal and pathological brain. In the peripheral nervous system, GLS1 expression and activity in the dorsal root ganglia have been proposed to generate glutamate pools involved in inflammatory pain, suggesting a role for glutaminase inhibitors in the modulation of nociceptor function (Hoffman et al. 2010). Finally, HIV-associated dementia has been linked to upregulation of glutaminase activity and subsequent glutamate excitoxicity derived from HIVinfected macrophages (Erdmann et al. 2007; Erdmann et al. 2009).

In 2013, Ferreira and coworkers published a detailed work describing the filamentous form of human glutaminase $\mathrm{C}$ (a short form of GLS1) (Ferreira et al. 2013) (Fig. 12c-e). They propose it to be the most active form of the enzyme, composed of a right handed double-stranded helix with seven tetramer copies per turn per strand and interacting via the $\mathrm{N}$-terminal domains ( $53 \pm 2 \mathrm{~nm}$ rise per turn, strand inclination of $25^{\circ}$, and an average width for a single strand of $6.6 \pm 0.7 \mathrm{~nm}$, estimated resolution of model is $35 \AA$ ). They identify a lysine which becomes acetylated in vivo that down-regulates the enzyme by disrupting the filament. Previous models of enzyme activation involved promotion of inactive dimers into active tetramers by binding to phosphate. However, the inhibitor BPTES stabilizes the tetrameric form with a rigidly open gating loop at the active site. Ferreira and coworkers found that BPTES stabilizes the tetramer but disrupts the filamentous 
a

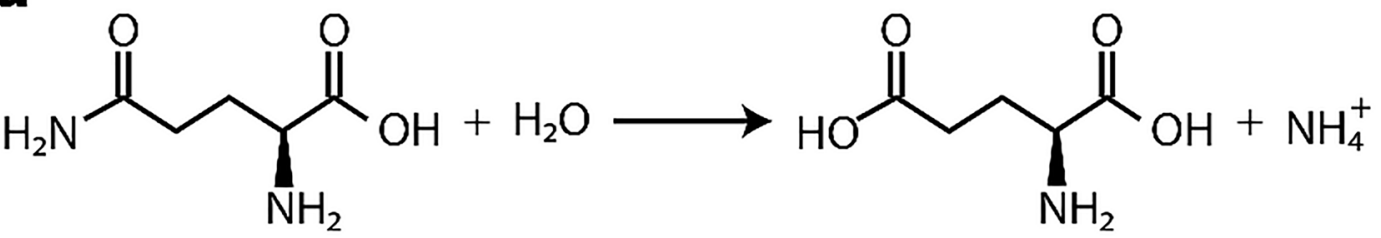

L-glutamine

b

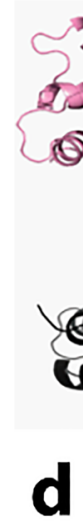

d

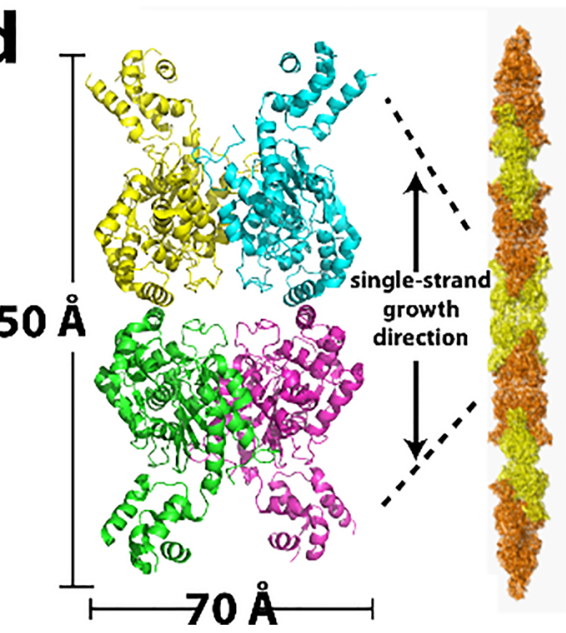

Fig. 12 Reaction catalyzed and structures formed from glutaminase. a Reaction catalyzed by glutaminase (EC 3.5.1.2). b Structure of full length human glutaminase bound to the inhibitory molecule BPTES (adapted with permission from DeLaBarre et al. 2011). c Negative stain TEM of crosslinked glutaminase filaments (scale bars represents $100 \mathrm{~nm}$ )

form, thereby explain the mechanism of inhibition as disruption of the filament (Ferreira et al. 2013). They also found that a mutation found in cancer cells (K325A) leads to enzyme much more prone to form filaments and therefore is hyperactivated.

\section{$\beta$-lactamase-like protein}

$\beta$-Lactamase-like protein (LACTB) is a $55 \mathrm{kDa}$ homologue of bacterial penicillin-binding proteins found in the intermembrane space of mitochondria of mammals (Smith et al. 2001; Peitsaro et al. 2008). Though its enzymatic activity is not
L-glutamic acid
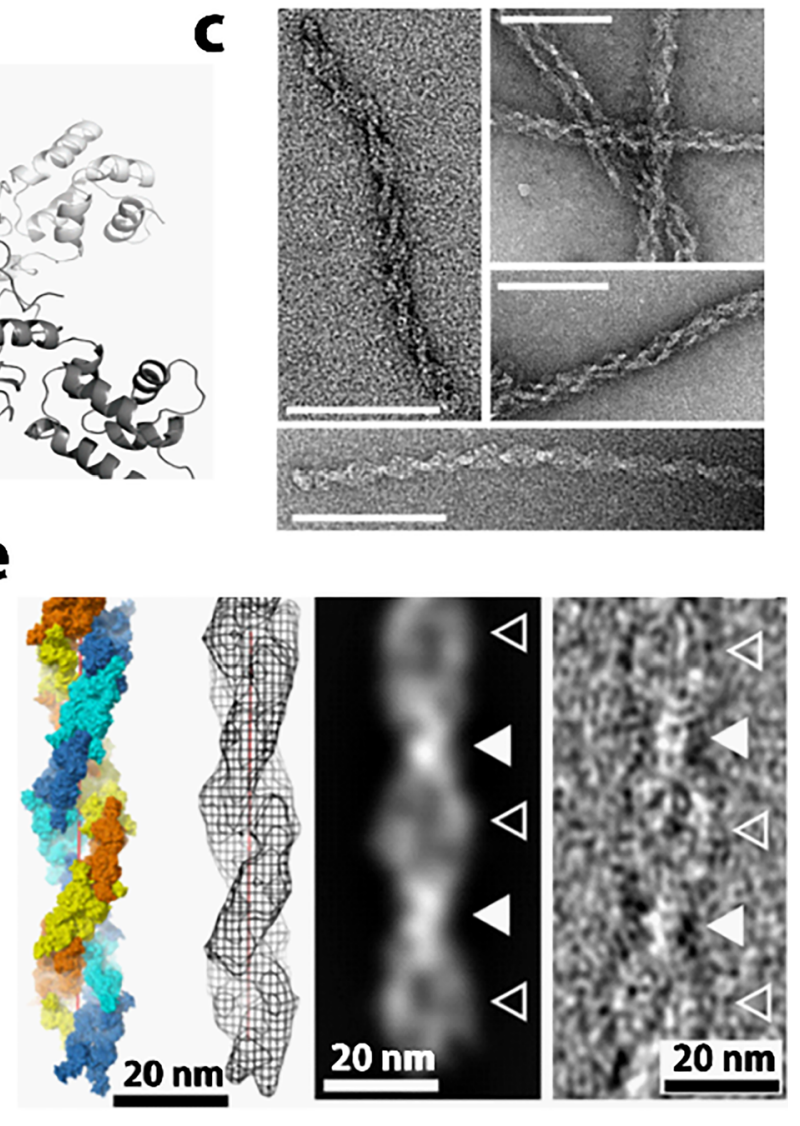

(adapted with permission from Ferreira et al. 2013). d A model of one of the two strands of the double helical glutaminase filaments (adapted with permission from Ferreira et al. 2013). e A model of the double helical filament of glutaminase (adapted with permission from Ferreira et al. 2013)

known, homologues are serine proteases and all important motifs of such enzymes are preserved in LACTB (Peitsaro et al. 2008). Studies have found a causal relationship between LACTB and obesity (Chen et al. 2008b). LACTB has been shown to polymerize into stable filaments both in vitro and in the mitochondrial intermembrane space (Fig. 13a). These may be important for submitochondrial organization and metabolon organization (Polianskyte et al. 2009). Analysis of the filaments suggests that LACTB forms tetramers that further oligomerize into the observed polymers. In mitochondria, the LACTB filaments are found tethered to the inner membrane but spanning intracristal regions of the 

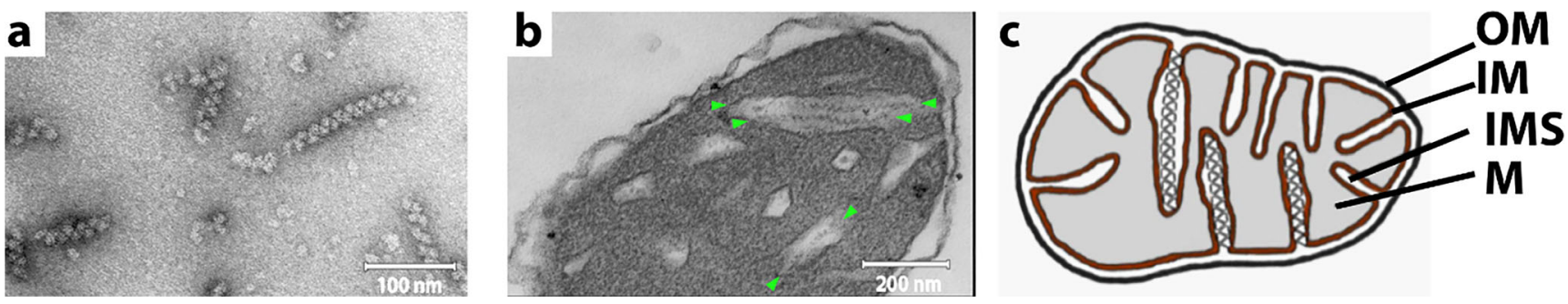

Fig. 13 a Filaments of purified LACTB viewed by negative stain EM (adapted with permission from Polianskyte et al. 2009). b Thin section of a rat liver mitochondrion with filaments in the cristal part of the intermembrane space (adapted with permission from Polianskyte et al. 2009). c Schematic of location of LACTB filaments in the intra-cristal part of the intermembrane space of mitochondria. IM, inner mitochondrial membrane; OM, outer mitochondrial membrane; IMS, intermembrane space; M, matrix (adapted with permission from Polianskyte et al. 2009) intermembrane space (Fig. 12b, c). The purpose of the filaments, or function of LACTB, is not currently known.

\section{2-Cys peroxiredoxins}

2-Cys peroxiredoxins (2CPrxs) are thioredoxin-dependent peroxidases that catalyze the reduction of hydrogen peroxide using conserved active-site cysteine residues (Fig. 14a). These enzymes are found in archea, bacteria, and eukaryotes. Hydrogen peroxide produced in cells can be toxic although it is also an important second messenger; hence, its levels must be tightly controlled (Puerto-Galan et al. 2013; Noichri et al. 2015). A functional switch from the detoxification/ peroxiredoxin activity to ATP-independent molecular chaperone activity can be triggered by oxidative stress, site-specific phosphorylation, or high temperature in many $2 \mathrm{CPrxs}$ (Angelucci et al. 2013) (Fig. 14a). Both states have a role in human disease, from resistance to neurodegenerative disorders to host-pathogen interactions, up to the onset of cancer (Moon et al. 2005; Cimini et al. 2013). Foci of 2CPrx form in mouse epithelial cells under oxidative stress (Puerto-Galan et al. 2013) (Fig. 14b).

Reduced 2CPrx forms homodimers that can associate into toroidal decameric rings during the peroxiredoxin catalytic cycle (Fig. 14c) (Wood et al. 2002; Noichri et al. 2015). Hyper-oxidation triggers stacking of decamers into filaments, which stabilizes the decameric structure (Fig. 14d), simulated by substitution of active site cysteines with serine (Fig. 14e, f) (Gourlay et al. 2003; Angelucci et al. 2013; Noichri et al. 2015). The filaments appear as tubes with a central cavity that binds unfolded proteins (Saccoccia et al. 2012; Teixeira et al. 2019). Figure $14 \mathrm{~g}$ shows a theoretical model of filamentous 2CPrx based on x-ray crystal structures of the decameric ring forms of the enzyme (Saccoccia et al. 2012). Hence, 2CPrxs are also redox sensitive holdases, responding to redox stress by filament formation which then sequesters unfolded proteins and thereby prevents protein aggregation (Kumsta and Jakob 2009).

\section{Ribonucleotide reductase}

Ribonucleotide reductases (RNRs) are responsible for all de novo biosynthesis of DNA precursors in nature by catalyzing the conversion of ribonucleotides to deoxyribonucleotides (Fig. 15a). Hence, human RNRs are targets for anti-cancer drugs. Both class 1a (from human) and class $1 \mathrm{~b}$ (from Bacillus subtilis) RNRs have been shown to form various oligomeric forms, including monomers, dimers, hexamers, and filaments (Ando et al. 2016; Thomas et al. 2019) (Fig. 15b). Punctate foci of RNR (both subunits, Rnr2 and Rnr4) were also discovered in yeast upon nutrient starvation (Narayanaswamy et al. 2009). The B. subtilis RNR is composed of $\operatorname{NrdE}$ and $\operatorname{NrdF}$ ( $\alpha$ and $\beta$ subunits, respectively), both being required for activity. The structures of various oligomeric states have been investigated for the class $1 \mathrm{~b}$ RNR from $B$. subtilis by SAXS, x-ray crystallography, and cryoEM (Thomas et al. 2019). Three distinct dimers (NrdE S type and I type dimers, and NrdF dimers) were characterized by $\mathrm{x}$ ray crystallography (Fig. 15c) (Thomas et al. 2019). Two filamentous forms were investigated using cryo-EM to 4.7-4.8 $\AA$ resolution (Fig. 15d, e) (Thomas et al. 2019). Both occur under conditions of enzyme inhibition, namely high $(100 \mu \mathrm{M})$ concentrations of dATP (Thomas et al. 2019). Considering only the NrdE subunit of RNR, a right-handed double helical filament is found which contains alternating dimeric interfaces (Fig. 15d, closeups of I and S type dimers shown in Fig. 15ci, iii). The presence of the NrdF dimers results in a filament with a single NrdE filament bound by NrdF dimers (Fig. 15e). A model for the allosteric regulation of RNR is shown in Fig. 16.

\section{Recombinase A}

Recombinase A (RecA) (and its eukaryotic homologue Rad51) are responsible for strand exchange in homologous recombination, a process important for DNA repair, viral integration, and recombination of DNA segments between homologous DNAs as in meiosis (Lusetti and Cox 2002). These functions have roles in generating genetic diversity and preventing DNA damage that could otherwise result in diseases such as cancer. RecA 

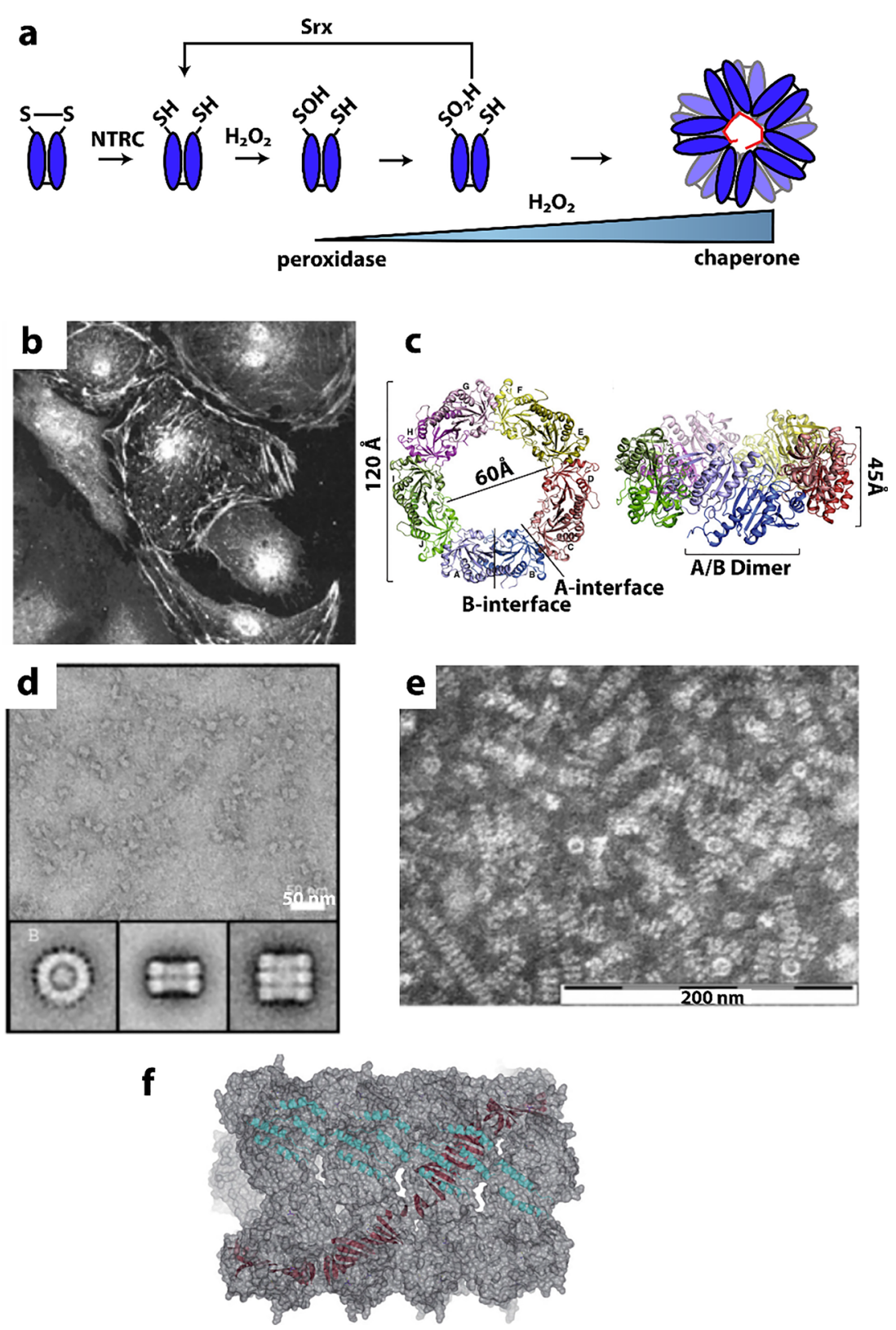

Fig. 14 Reactions catalyzed and structures formed from 2-Cys peroxiredoxin (2CP). a Under oxidant conditions, the sulfenic acid intermediate of the peroxidatic cysteine residue may be further oxidized to sulfinic acid. The reduction of the enzyme, which is most efficiently performed by NADPH-dependent thioredoxin reductase C (NTRC), is a pre-requisite for sulfenic acid formation and, thus, for overoxidation. Srx is able to catalyze the reversion of the overoxidized to the reduced form of the enzyme. Therefore, the redox status of chloroplast 2-Cys Prxs is highly dependent of NTRC and Srx. The quaternary structure of 2-Cys Prxs determines the activity of these enzymes. In the reduced form, the enzyme is a dimer and shows peroxidase activity; overoxidation as occurs in oxidative stress favors the formation of the decameric form, which lacks peroxidase activity, can stack into filaments, and shows chaperone activity (adapted with permission from Puerto-Galan et al. 2013). b
Synchronized C19 mouse epithelial cells treated with glucose oxidase then stained for 2CPrx (adapted with permission from Phalen et al. 2006). c Toroidal decamer of Schistosoma mansoni 2CPrx (adapted with permission from Saccoccia et al. 2012). d Negative stain EM micrograph of bovine CPrx showing stacked rings (adapted with permission from Gourlay et al. 2003). e Negative stain TEM of recombinant C48S mutant of CPrx from Schistosoma mansoni that results in constitutive chaperone activity (and constitutive filament formation) (adapted with permission from Angelucci et al. 2013). f Negative stain EM micrograph of bovine C47S CPrx showing filaments (adapted with permission from Gourlay et al. 2003). g Theoretical model of filamentous 2CPrx from stacked decameric rings. Cyan and red show alpha and beta structures, respectively, from subunits of each ring and their relative orientations (adapted with permission from Saccoccia et al. 2012) 
a
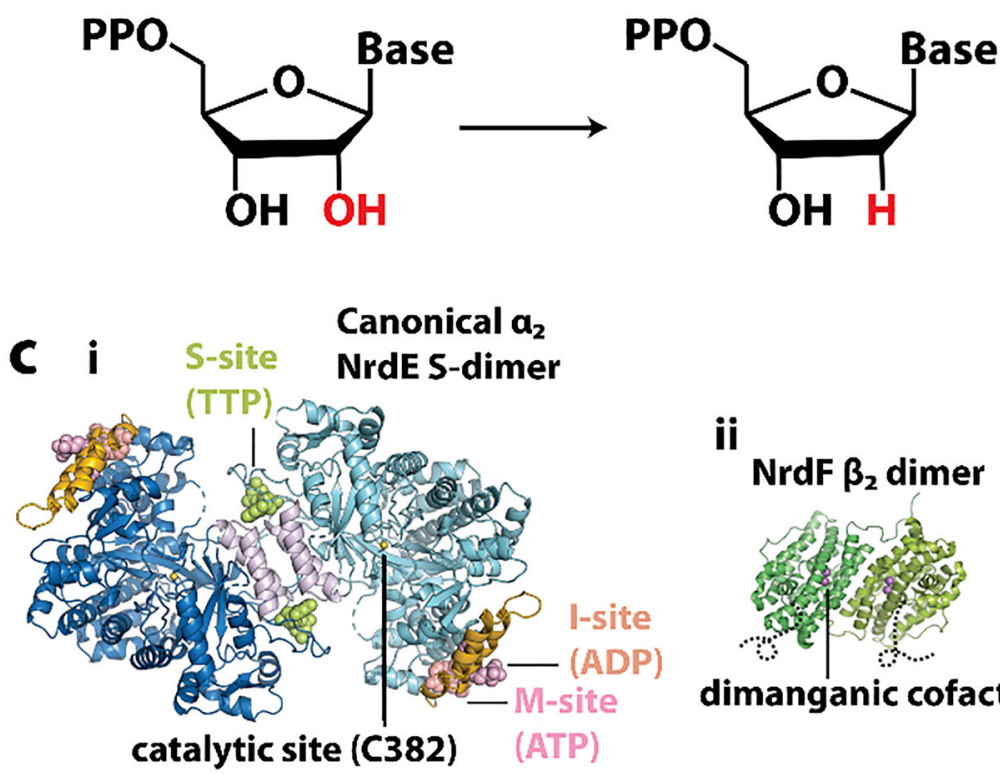

ii

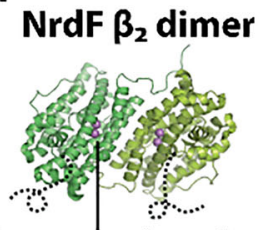

dimanganic cofactor

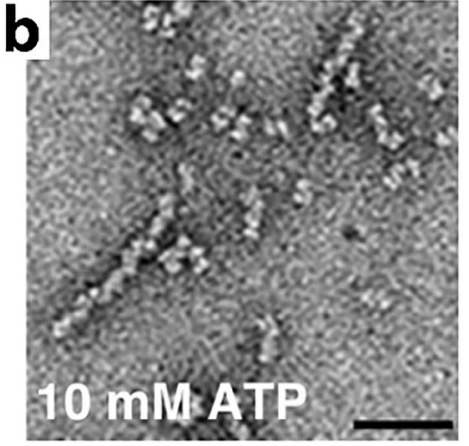

iii Canonical $a_{2}$ NrdE I-dimer

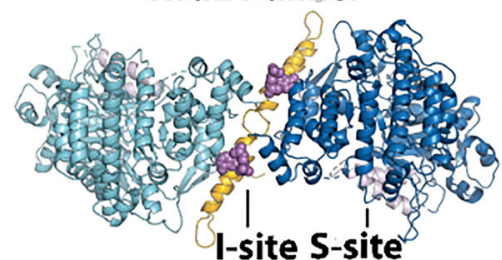

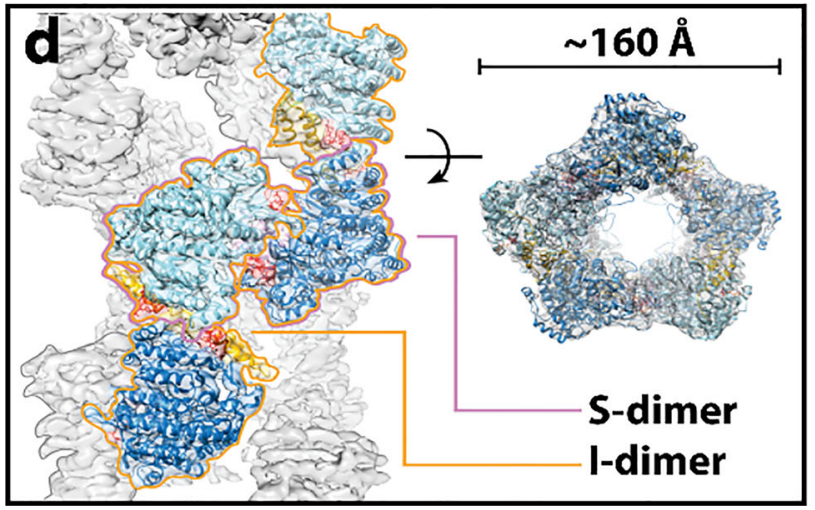

Fig. 15 a Reaction catalyzed by ribonucleotide reductase (EC 1.17.4.1). b Negative stain TEM showing filaments of human RNR. Scale bar represents $50 \mathrm{~nm}$ (adapted with permission from Ando et al. 2016). c $\mathrm{X}$-ray crystallographic structures of $B$. subtilis RNR dimers. i S-type dimer of $\operatorname{NrdE}$ ( $\alpha$ subunit of RNR). ii I-type dimer of $\mathrm{NrdE}$ ( $\alpha$ subunit

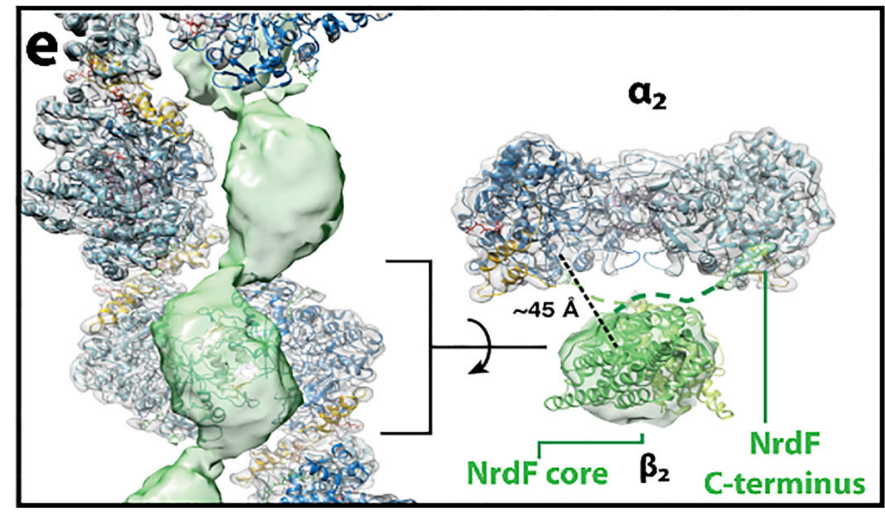

of RNR). iii Dimer of NrdF ( $\beta$ subunit $r$ RNR) (adapted with permission from Thomas et al. 2019). d Cryo-EM structure of $B$. subtilis RNR filament of NrdE bound to dATP (adapted with permission from Thomas et al. 2019). e Cryo-EM structure of B. subtilis RNR filament of NrdE and $\mathrm{NrdF}$ with dATP (adapted with permission from Thomas et al. 2019)

structures, but the filament can also assume a separate, inactive structure, which was also characterized to $4.3 \AA$ resolution (Chen et al. 2008a). In all cases, the filaments are righthanded with straight helical axes, $\sim 6$ RecA per turn and $94 \AA$ pitch. The DNA is bound inside the helix formed by the RecA proteins, closer to the helical axis. The ATP analogue binds at the interface between adjacent RecA protomers, explaining the ATP dependency of DNA binding and the dissociation from DNA upon ATP hydrolysis. The DNA is stretched globally, but adopts B-DNA-like conformation in the three nucleotides (or base pairs) bound to each RecA protomer, with stretching between. This stretching is thought to destabilize the donor duplex allowing local melting and sampling of base pairing by the original single stranded DNA (Chen et al. 2008a). 


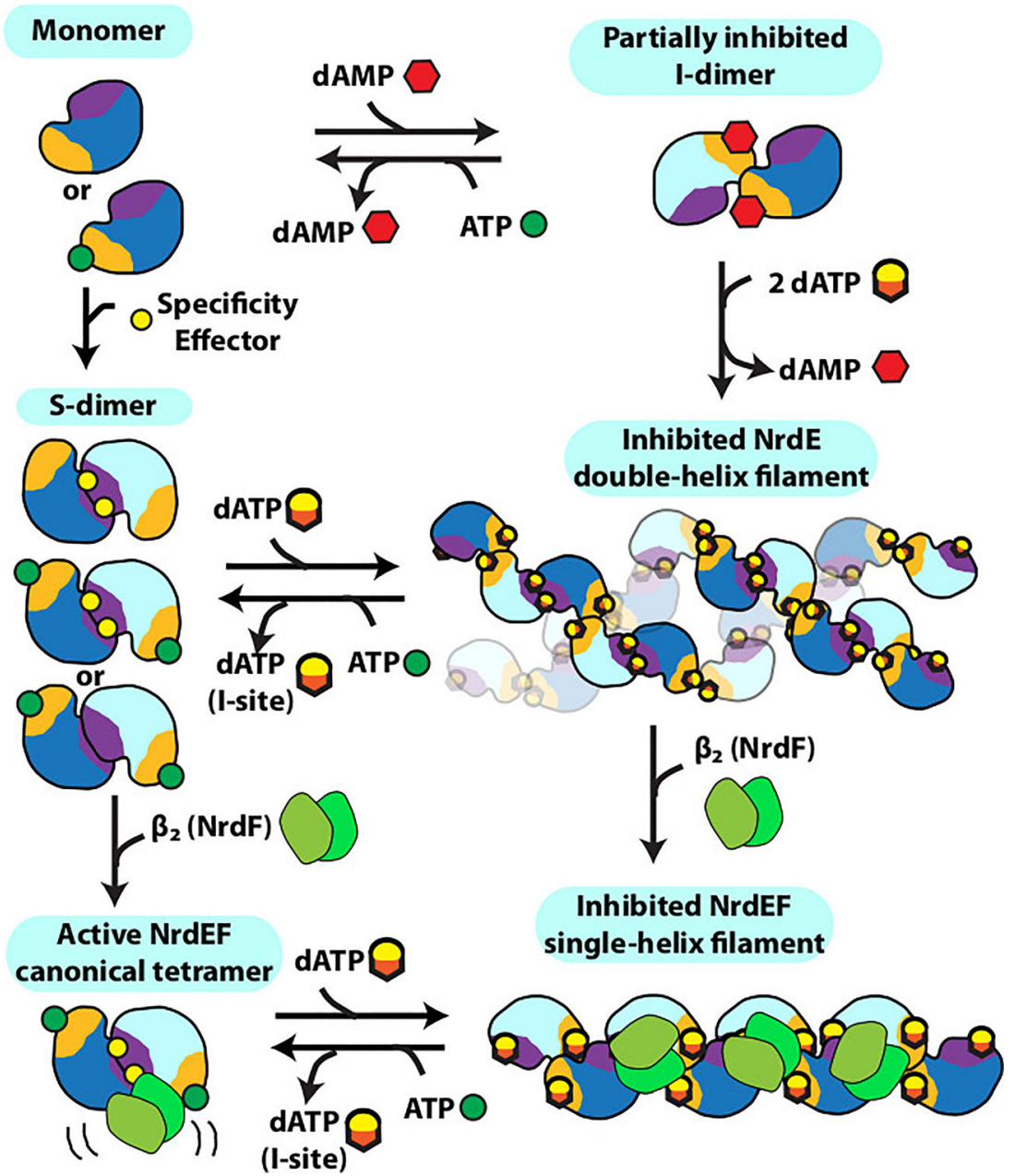

Fig. 16 Model for allosteric regulation of $B$. subtilis RNR. Without nucleotides, NrdE is a monomer, but dAMP and dATP can bind the Isite and induce a partially inhibited I-dimer. Addition of specificity effectors (dATP, TTP, or dGTP) instead induces the monomer to form an Sdimer. When specificity effectors (including dATP) bind to the I-dimer, they induce formation of an inhibited double-helical NrdE filament composed of alternating S- and I-dimer interfaces. NrdF competes for the NrdE double-helical interface, and thus, NrdF binding leads to the dissociation of the NrdE double-helix into an individual helical structure.

\section{Chromosomal replicator initiator protein (DnaA)}

DnaA and its homologues in bacteria are required for the initiation of replication (Kaguni 2006; Katayama et al. 2010; Leonard and Grimwade 2010). DnaA possesses a sequence specific DNA binding domain, which recognizes specific sequences in the bacterial origin, and is also a AAA+ ATPase which binds and hydrolyzes ATP. DnaA binds to the sequences at the origin and is responsible for inducing strand opening (Fig. 18a) at a nearby sequence, which then allows for loading of the replicative helicases DnaB (Fig. 18b) (Bleichert et al. 2017). The crystal structure of DnaA bound to single stranded DNA shows a right handed helical filament that binds DNA on the interior of the spiral, near the helical axis (Duderstadt et al. 2011)
Binding of NrdF to the NrdE filament leads to an inter-subunit gap that is too large to allow for radical transfer. Both the NrdE and NrdEF filaments are reversible by addition of ATP, which can displace dATP from the I-site and induce dissociation of the I-dimer interface. Finally, addition of $\mathrm{NrdF}$ to the S-dimer leads to formation of an active but asymmetric $\alpha 2 \beta 2$ tetramer in which a hinge motion between the two subunits plays an important role in activity (adapted with permission from Thomas et al. 2019)

(Fig. 18c), reminiscent of the structure formed by RecA (Fig. 18d). Similar to the mechanism of RecA, the DNA is stretched from B-DNA helical parameters which is thought to be important for destabilization of the double helix. The ATPase domain is responsible for proteinprotein interactions forming the spiral, as well as making non-sequence specific contacts to the DNA (Fig. 18b). The analogue of ATP found in the crystal structure binds at the interface between neighboring DnaA protomers, similar to ATP binding to RecA filaments. The helical filament contains 8 copies of the single chain DnaA protomer per turn $\left(45^{\circ}\right.$ per protomer), with a pitch of $178 \AA$. Also like RecA, this filament is polar, meaning the two ends of the filament are not equivalent (Duderstadt et al. 2011). 
Fig. 17 Reaction catalyzed and structures formed by $\operatorname{Rec} A / \operatorname{Rad} 51$. a Reaction of DNA strand exchange catalyzed by $\operatorname{RecA} / \operatorname{Rad} 51$. b Negative stain electron micrograph of human Rad51 with single stranded DNA, $\mathrm{ADP}$, and $\mathrm{AlF}_{4}^{-}$(adapted with permission from Yu et al. 2001). c Helical reconstruction of filament shown in B (adapted with permission from Yu et al. 2001). d X-ray crystal structure of $E$. coli RecA bound to single stranded DNA, ADP, and $\mathrm{AlF}_{4}^{-}$(adapted with permission from Chen et al. 2008a)
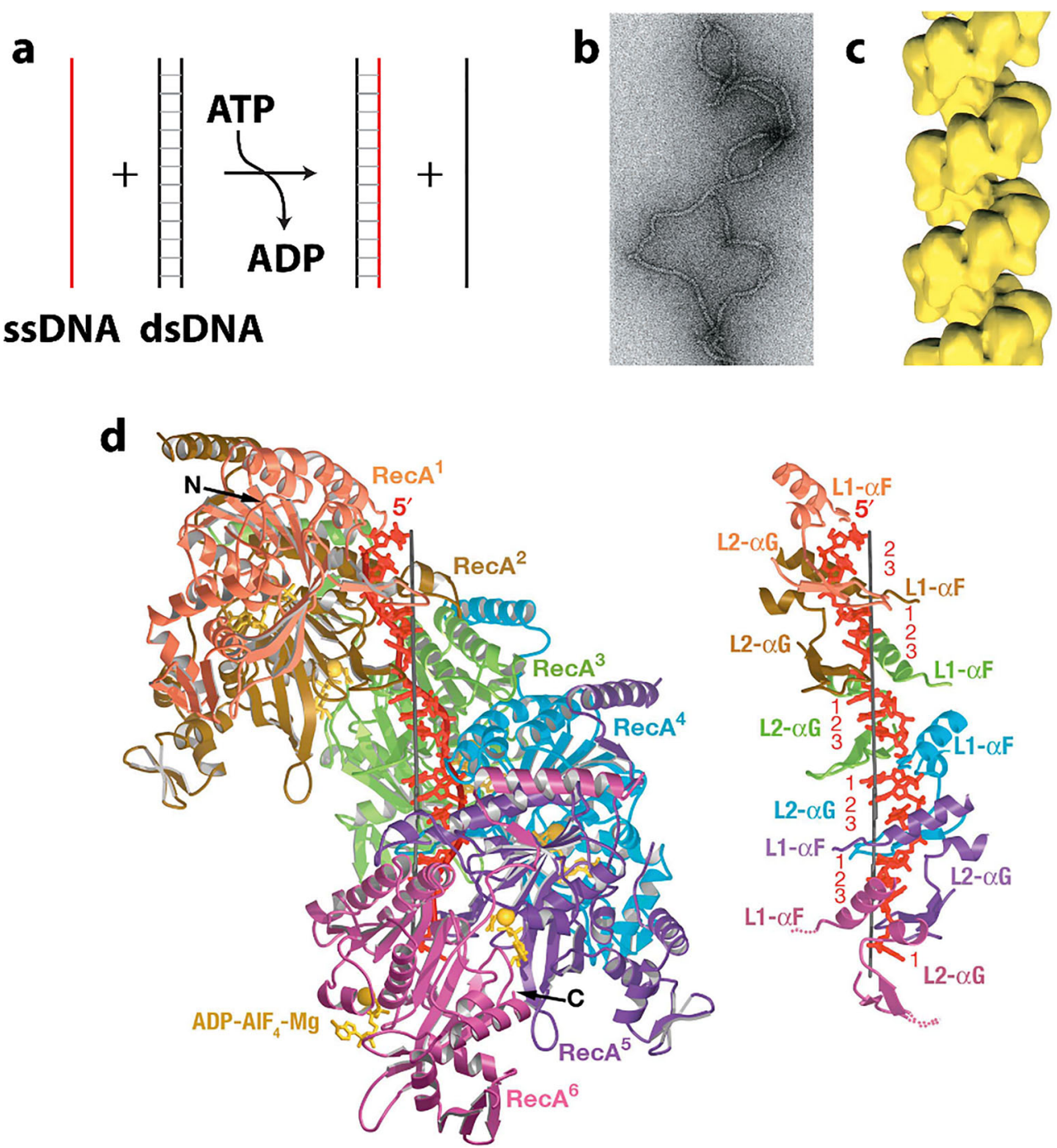

\section{SgrAl restriction enzyme (SgrAl)}

SgrAI is a type II restriction endonuclease from the bacterium Streptomyces griseus (Tautz et al. 1990). Restriction endonucleases protect their host cells from invading DNA, such as the bacterial viruses known as phage (Pingoud et al. 2014). They are also considered to be coded by "selfish DNA" as their genes, as well as those for their cognate methyltransferases and possibly other related proteins have been shown to evolve through horizontal gene transfer from organism to organism (Kobayashi 1996). They not only impart this benefit of innate immunity, but also other functions including genetic recombination, nutrition, and genome evolution among others (Vasu and Nagaraja 2013). Like most type II restriction endonucleases, SgrAI cleave particular sequences in double stranded DNA in a $\mathrm{Mg}^{2+}$-dependent reaction (Fig. 19a) (Tautz et al. 1990). The primary DNA recognition sequence of SgrAI is shown in Fig. 19b, CRCCGGYG, where R represents A or G and $\mathrm{Y}$ represents $\mathrm{C}$ or $\mathrm{T}$ (Tautz et al. 1990). SgrAI also cleaves a second type of site, secondary sites, with sequences CRCCGGGG or CRCCGGY(A or C or T) (Bitinaite and Schildkraut 2002). However, these are only cleaved if the primary site is also present, either on the same contiguous molecule of DNA, or at high concentrations (Bilcock et al. 1999; Bitinaite and Schildkraut 2002; Daniels et al. 2003; HingoraniVarma and Bitinaite 2003; Wood et al. 2005). SgrAI also cleaves primary site DNA faster when there is more than one copy on the same contiguous DNA (or in high concentrations).

SgrAI forms filaments when bound to primary site DNA sequences (Park et al. 2010; Lyumkis et al. 2013; Ma et al. 2013), and its structure has been characterized to $3.5 \AA$ resolution by helical reconstruction and cryo-electron microscopy (Polley et al. 2019). The filament is a left-handed helix with approximately 4 copies of the SgrAI/DNA complex per turn (Fig. 19c). It is stabilized by protein-protein and protein-DNA interactions between neighboring SgrAI/DNA complexes. Compared to the structure of non-filamentous SgrAI, a $\sim 10^{\circ}$ rotation is found between the two chains of the SgrAI dimer 
Fig. 18 Reaction catalyzed and structures formed by $E$. coli DnaA. a ATP-dependent DNA duplex opening reaction catalyzed by DnaA. b Filament formation by DnaA on the E. coli origin of replication results in strand separation facilitating replicative helicase loading (adapted with permission from Bleichert et al. 2017). c X-ray crystal structure of Aquifex aeolicus DnaA bound to single stranded DNA (adapted with permission from Duderstadt et al. 2011)

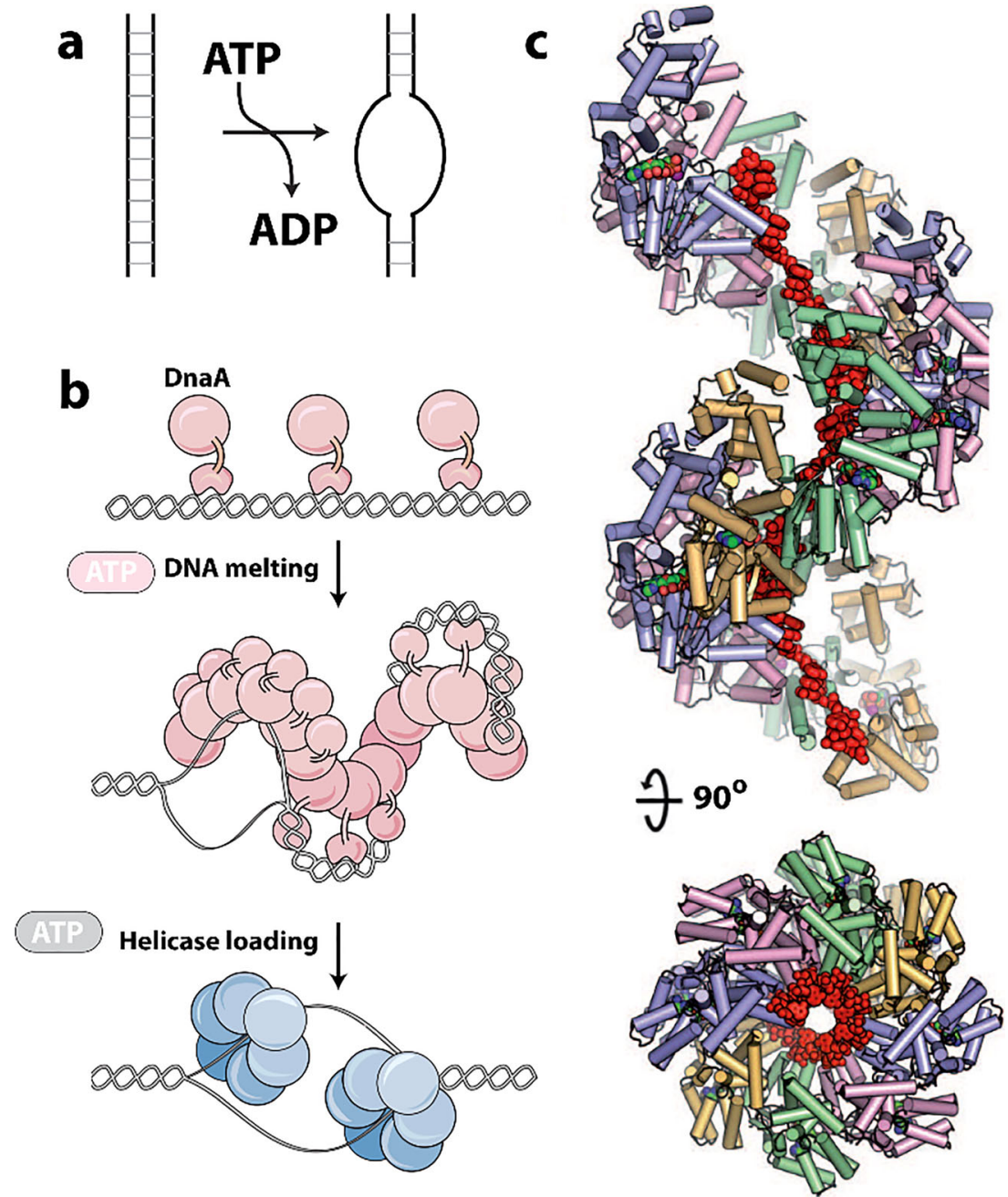

(Polley et al. 2019). In the structure of the non-filamentous state, determined by x-ray crystallography, one of the two $\mathrm{Mg}^{2+}$ is displaced from its expected position, providing an explanation for the low DNA cleavage activity (Dunten et al. 2008; Little et al. 2011). To accommodate the rotation between subunits in the filamentous conformation, many residues at the dimeric interface shift position which is hypothesized to stabilize a more optimal position of the misplaced $\mathrm{Mg}^{2+}$ (Polley et al. 2019).

The working model for filament formation and activation of SgrAI is shown in Fig. 19d (Polley et al. 2019). SgrAI bound to DNA is in equilibrium between two conformations, a low activity $\mathrm{T}$ state and a high activity $\mathrm{R}$ state. Only the R state forms filaments. Filament formation in turn stabilizes the $\mathrm{R}$ state. In the absence of filament formation, the $\mathrm{T}$ state is favored; however, the R state is populated sufficiently to selfassemble into filaments under certain conditions. When bound to the primary site, the $\mathrm{R}$ state is sampled sufficiently to form filaments if concentrations are also sufficient (Fig. 19e, top). When bound to the secondary site, the R state is less favored, and therefore, filamentation is far less favored and secondary sites will not be cleaved (Fig. 19e, middle). Yet in the presence of filaments of SgrAI bound to the primary site, SgrAI bound to secondary sites will join, upon occasional sampling of the $\mathrm{R}$ state (Fig. 19e, lower). Structural studies suggest that the reason the $\mathrm{R}$ and $\mathrm{T}$ states differ energetically with the two sequences is due to sequence-dependent DNA structural energetics. Base stacking is different in the $\mathrm{R}$ state conformation (in the filament), which is predicted to favor primary site sequences. Hence, the origin of the differential DNA cleavage activity on the two types of sequences, primary and secondary, may originate in DNA structure and energy with filamentation serving as the means to detect this energy. In the filament, SgrAI cleaves both types of sites rapidly resulting in a 200 fold acceleration in the case of primary sites, 1000-fold in the case of secondary (Shah et al. 2015). The expansion of DNA cleavage activity from primary to primary and secondary increases the number of cleavage sequences from 3 to 17 .

Global kinetic modeling of filament association, dissociation, and DNA cleavage FRET reaction data indicate that the rate limiting step of the activated DNA cleavage reaction is filament association (Park et al. 2018a, b). The second order 
Fig. 19 Reactions catalyzed and structures formed by SgrAI. a, b Cleavage (hydrolysis) of double stranded DNA catalyzed by SgrAI, a type II restriction endonuclease (EC: 3.1.21.4). c (Left) Filament formed by SgrAI bound to primary site DNA, also known as a run-on oligomer. (Right) Three different views of a single SgrAI/DNA complex, the basic building block of the run-on oligomer filament. d Working model of activation via filament formation by SgrAI. e Working model for expansion of DNA sequence specificity of SgrAI in recruiting SgrAI bound to secondary site DNA sequences (gold balls) by filaments formed by SgrAI bound to primary sites (blue balls) a

b
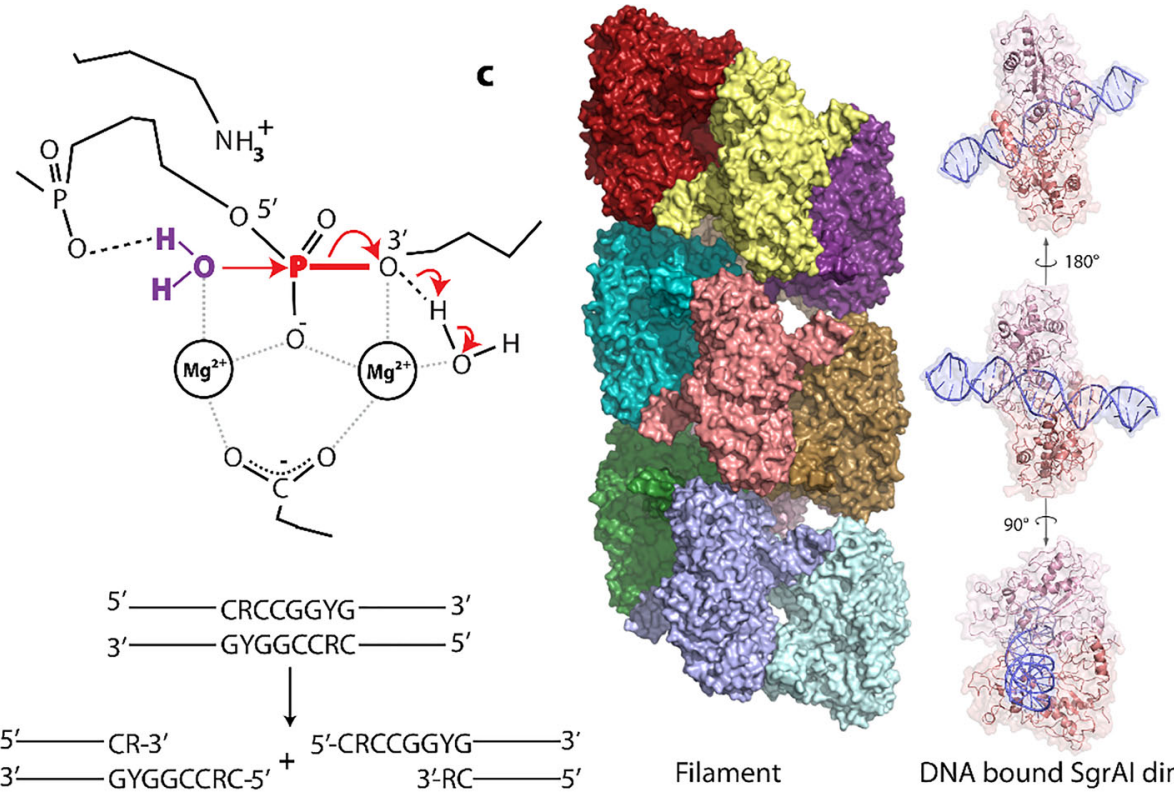

Filament

DNA bound SgrAl dimer

d

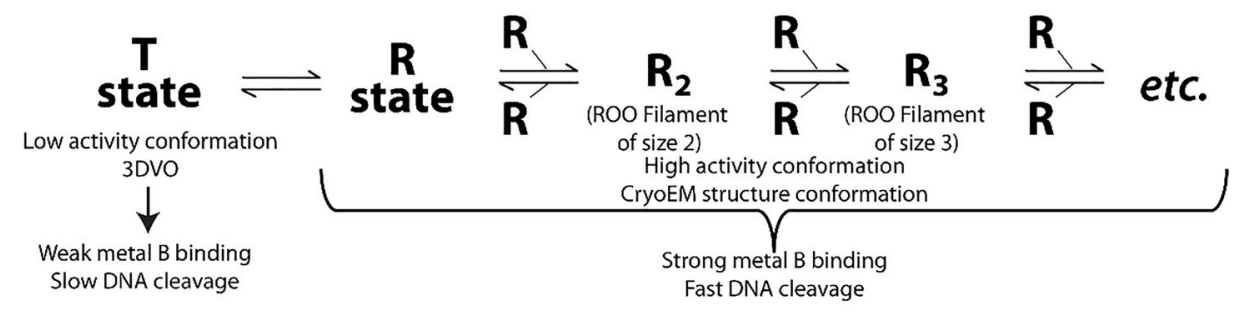

e

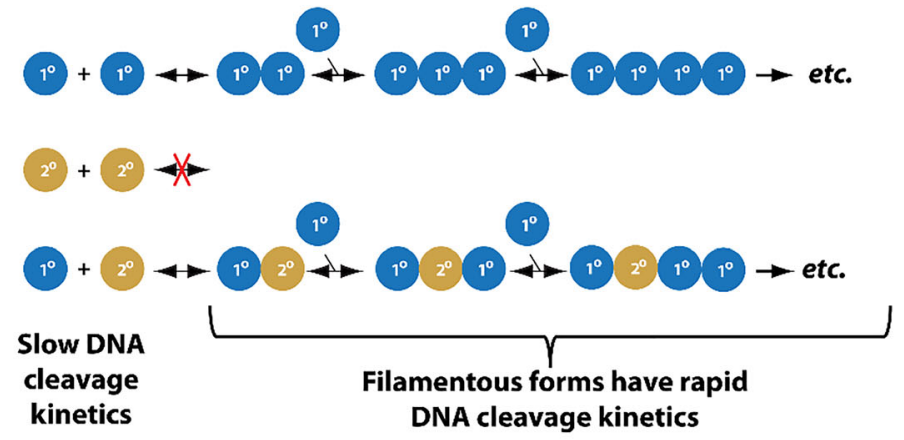

Sgral bound to primary site

Sgral bound to secondary site

rate constant is several orders of magnitude lower than diffusion limited (i.e., $10^{5} \mathrm{M}^{-1} \mathrm{~s}^{-1}$ ). Hence, to overcome this slow association rate constant, high concentrations $(100 \mathrm{nM}$ or greater) of SgrAI/DNA are needed (and in the R state conformation). In the cell, this limitation is overcome by the local concentration effect of recognition sequences on the same contiguous DNA (Barahona et al. 2019). Hence, sites on the same DNA appear to be at a higher concentration, relative to each other, than they actually are in the cell and are more likely to form the filament, stabilize the activated conformation of SgrAI, and become cleaved. SgrAI bound to sites on separate DNA molecules are far less likely to associate into a filament, since concentrations of DNA in the cell are low $(\sim 3 \mathrm{nM})$. Primary sites in the host DNA are protected by methylation, but secondary sites are not. SgrAI will not bind to methylated primary sites, but does bind to the secondary sites tightly (nanomolar affinity). Invading DNA will contain both types of sites, unmethylated, and SgrAI in the cell binds both types. SgrAI bound to the primary sites induces the filament, which then draws in SgrAI bound to secondary sites, but only those on the same DNA molecule, owing to the local concentration effect (Barahona et al. 2019). This effect then is useful to focus or sequester enzyme activity on only substrates of interest; in this case, the invading phage DNA and not the host DNA. 
The global kinetic model also allowed simulations to be performed to predict SgrAI behavior under in vivo conditions (Barahona et al. 2019). Compared to a non-filament mechanism (Binary, with all the same rate constants but limited to an assembly of 2 enzymes, Fig. 20), the Filament-forming mechanism is superior in both speed (e.g., the rate of DNA cleavage), as well as the ability to sequester its damaging DNA cleavage activity on invading phage DNA (and away from host DNA, Figs. 19 and 20). Figure 20a shows why the filament mechanism is superior in speed over the Binary. The filament mechanism has multiple ways for enzymes to assemble (Fig. 20a, left), whereas the binary mechanism has only a single association configuration (Fig. 20a, center). Figure 20b shows that the filament mechanism is superior to the binary mechanism in cleavage of secondary sites (golden boxes, Fig. 20b), because no competition occurs between enzymes bound to the two types of sites. SgrAI bound to primary sites (blue boxes, Fig. 20b) induce filament formation, and SgrAI bound to secondary sites (gold boxes, Fig. 20b) join, leading to rapid DNA cleavage of all sites (Fig. 20b, upper). In the case of the binary mechanism, association of enzyme bound to primary sites is preferred (Fig. 20b, lower), and outcompete association with secondary site bound enzymes, and are therefore cleaved far slower than in the filament mechanism (compare red to green line, Fig. 20b, right).

The filament mechanism is also superior in sequestering enzyme activity (Fig. 21). In this figure, the in vivo role of SgrAI is depicted (Fig. 21a-c). SgrAI binds to primary and secondary sites on the phage DNA (Fig. 21a) and form the filament with activated DNA cleavage (Fig. 21b). However, due to the slow association rate constant of enzymes into the filament (i.e., $10^{5} \mathrm{M}^{-1} \mathrm{~s}^{-1}$ ), and the intrinsically low concentration of DNA in the cell (estimated at $3 \mathrm{nM}$ ), but high local concentration of SgrAI on the same contiguous DNA (estimated > 300 times higher (Barahona et al. 2019)), SgrAI bound to secondary sites on the host DNA (blue arrow, Fig. a Filamentous Assembly:

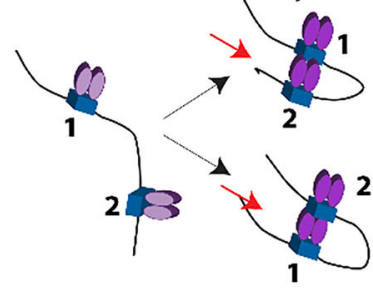

b Filamentous Assembly:

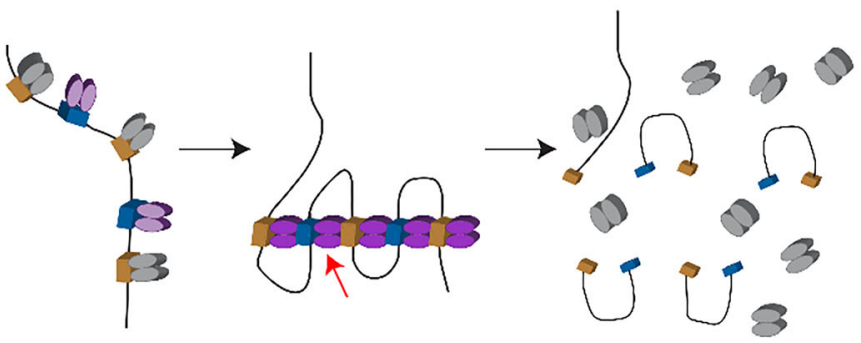

Binary Assembly:

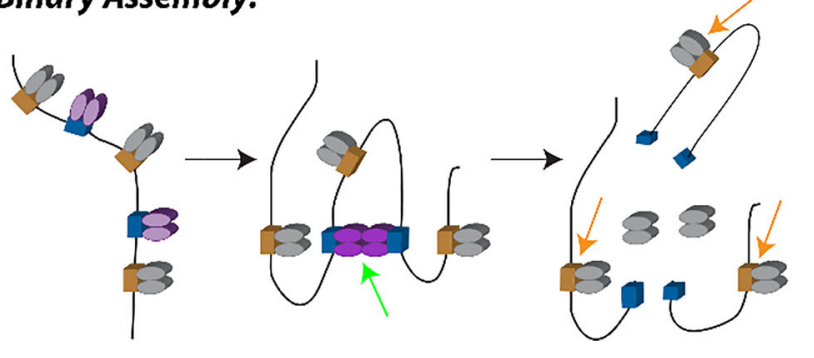

Binary Assembly:

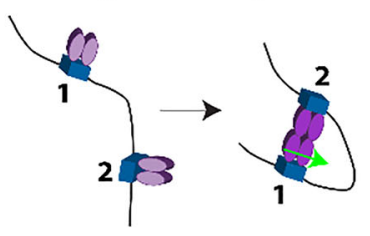

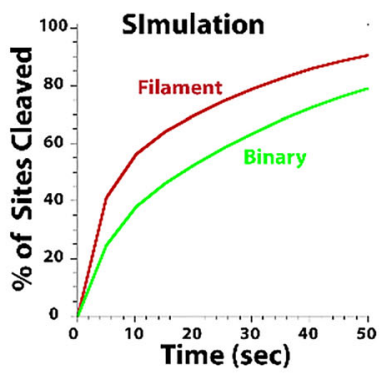
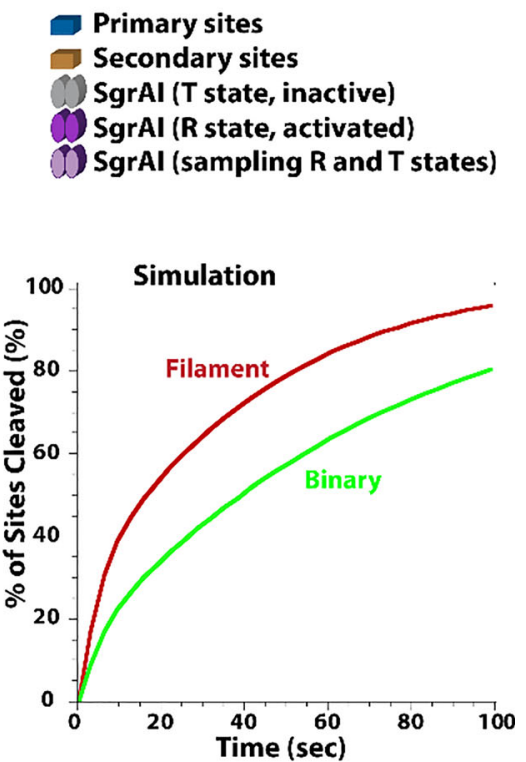

Fig. 20 The filament mechanism of SgrAI is superior to a non-filament, binary mechanism (forming a complex of 2 enzymes) in both speed and cleavage of secondary sites. a The greater speed derives from multiple ways enzymes can associate in the filament (left, red arrows) compared to a finite, discrete, binary non-filament model (middle, green arrow). Simulations show the greater enzymatic activity of the filament mechanism (red) over the non-filament or binary model (green) (right). b The filament mechanism is superior in cleavage of secondary sites as well, since these are out-competed compared to primary sites in the binary model (lower, green and gold arrows), but do not compete in the filament model as more enzymes can always add to either end (top, red arrow) 


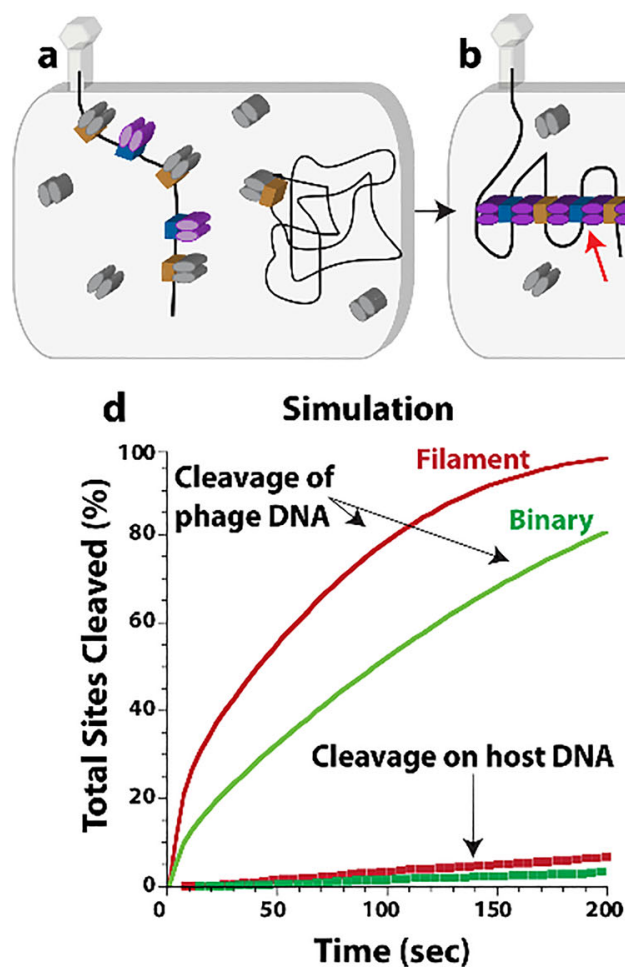

Fig. 21 The filament mechanism is superior in sequestration of secondary site cleavage activity. a SgrAI is inactive (grey) and binds to secondary sites (gold boxes) in the host DNA (right) and both primary (blue boxes) and secondary sites in the invading DNA. While bound to primary sites, SgrAI equilibrates between active and inactive states (partial purple color). b SgrAI in the active conformation (purple) associates into a filament (red arrow), drawing in SgrAI bound to secondary sites, inducing their activation (purple color), and resulting in DNA cleavage. $\mathbf{c}$ Both primary and secondary sites are cleaved on the invading DNA, but secondary sites

21b) do not assemble into the filaments (rate of association = $10^{5} \mathrm{M}^{-1} \mathrm{~s}^{-1} \times 3 \mathrm{nM} \times 3 \mathrm{nM}=0.0009 \mathrm{nM} / \mathrm{s}$ ), while those on the same contiguous DNA as the primary site (i.e., the phage DNA, red arrow, Fig. 21b) do (rate of association $=10^{5} \mathrm{M}^{-1}$ $\left.\mathrm{s}^{-1} \times 1 \mu \mathrm{M} \times 1 \mu \mathrm{M}=100 \mathrm{nM} / \mathrm{s}\right)$. Hence, secondary sites on the host DNA, which are not methylated by the SgrAI methyltransferase, are largely protected from damaging DNA cleavage. Concurrently, secondary sites on the phage are cleaved, which is expected to increase the effectiveness against phage transcription and replication (Fig. 21c). Simulations performed with the two mechanisms, having identical rate constants, show this effect (Fig. 21d-e). Cleavage on the host is simulated with DNA concentrations of $3 \mathrm{nM}$, while that on the phage is estimated at $1 \mu \mathrm{M}$, the estimated local concentration between primary and secondary sites on the same contiguous DNA, based on average expected distances between sites (Barahona et al. 2019). Both mechanisms effectively sequester their cleavage activity on the phage DNA, because of the slow association rate constants of enzymes into the assembly (filaments or binary assemblies) and the low concentrations of DNA in the cell (see "Cleavage on host DNA" compared to "Cleavage of phage DNA," Fig. 21d). However, the filament mechanism is faster (compare red to green line, "Cleavage of phage DNA," Fig. 21d). Yet if speed is required, a faster rate of binary assembly could instead be evolved, rather than a complicated filament forming mechanism with a slow association step. Figure 21e shows why the filament mechanism is yet superior; when the association rate constant for the assembly of the binary complex is increased so that its DNA cleavage rate matches that of the filament mechanism (a 4.5 -fold increase is necessary) (green solid line for "Cleavage of phage DNA," Fig. 21e), much more cleavage of secondary sites on the host DNA is predicted (blue arrow, Fig. 21e). The reason for this loss of sequestration is due to the much higher association rate constant necessary so that the DNA cleavage rate of the binary matches the speed of the filament mechanism (Barahona et al. 2019).

\section{The unfolded protein response nuclease kinase (IRE1)}

In eukaryotes, the vast majority of secretory and transmembrane proteins are folded in the endoplasmic reticulum (ER). 
Inositol requiring protein 1 or IRE1 (also known as Ire1) is a transmembrane ER protein that senses unfolded proteins in the ER (Wang and Kaufman 2016), and has both kinase and RNase (and splicing) enzymatic activities (Fig. 22a, b). The luminal domain of IRE1 senses the unfolded proteins by binding directly to them, as well as through the release of BiP (a protein that also binds unfolded proteins). This results in the assembly of IRE1 into dimers and higher order assemblies (Credle et al. 2005; Aragon et al. 2009; Korennykh et al. 2009; Gardner and Walter 2011; Walter and Ron 2011;
Karagoz et al. 2017), observed as foci in live imaging (Fig. 22c) (Aragon et al. 2009; Korennykh et al. 2009; Li et al. 2010; Ishiwata-Kimata et al. 2013). The assembly of IRE1 also allows for auto-phosphorylation via its cytosolic kinase domain, and consequential activation of its RNase activity in the assemblies (Korennykh et al. 2009) (Fig. 22d-f). The RNase activity has two consequences: non-conventional cytosolic mRNA splicing and regulated IRE1-dependent decay (RIDD) (Chen and Brandizzi 2013). The splicing involves two cleavages followed by ligation (by a separate RNA ligase)

\section{a}

HACI splicing

Cleavage site

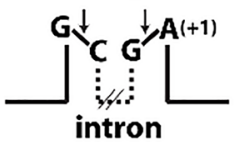

HAC ${ }^{u}$ mRNA $\downarrow \begin{gathered}\text { Ire1p } \\ \text { (endoribonuclease) }\end{gathered}$ $\stackrel{\text { G }}{2^{\prime}} \mathrm{P}_{\mathrm{OH}} \mathrm{L}^{\prime}$
XBP1splicing

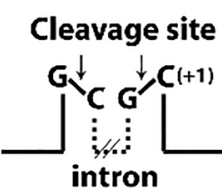

$X B P T^{\prime}$ mRNA
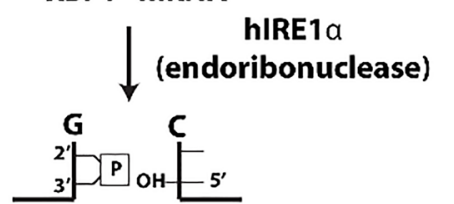

b
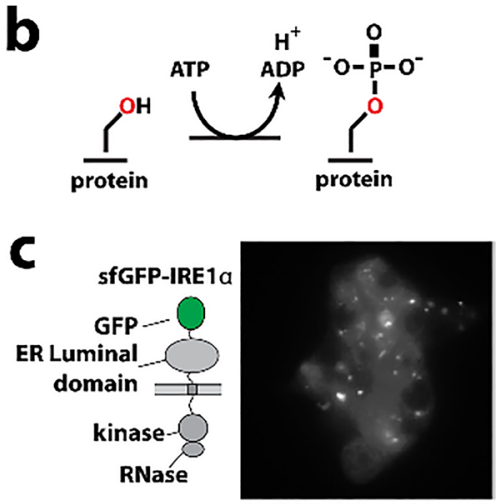

d

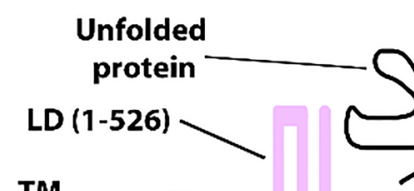

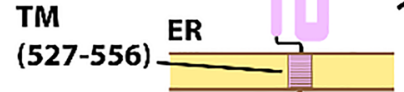

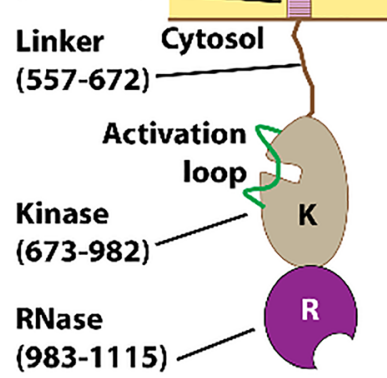

e

1. Binding of unfolded proteins

2. Oligomerization that activates RNase

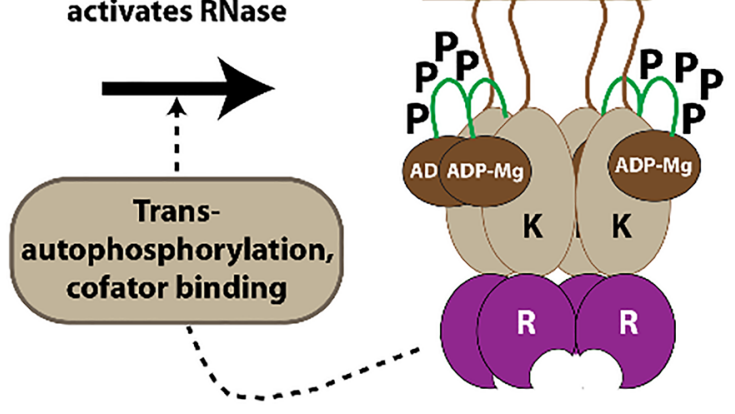

f

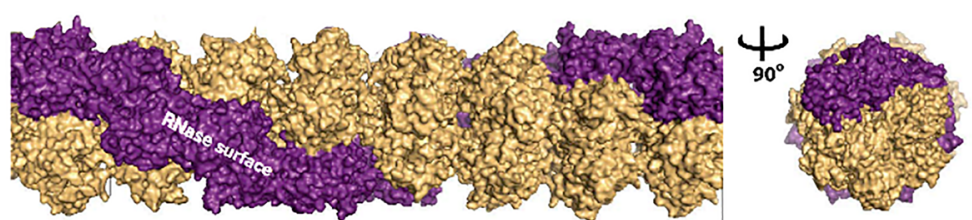

Fig. 22 Reactions catalyzed and structures formed by Ire1 (or hIRE1). a Splicing/RNase activity of Ire1/IRE1 $\alpha$ (adapted with permission from (Poothong et al. 2017)). b ATP-dependent phosphorylation of protein side chains via kinase activity (IRE1 autophosphorylates by phosphorylating adjacent IRE1 chains when oligomerized). $\mathbf{c}$ Human IRE1 $\alpha$ forms foci in cells upon addition of the ER stress agent DTT (adapted with permission from Ghosh et al. 2014). d Schematic of IRE1. The kinase domain of
IRE1 is colored light brown; the RNase domain is colored purple. TM, transmembrane domain (adapted with permission from Korennykh et al. 2009). e The splicing/RNase activity of IRE1 is activated upon oligomerization, induced via unfolded protein binding in the ER lumen (adapted with permission from Korennykh et al. 2009). f Two orthogonal views of the filament formed by IRE1, determined via x-ray crystallography (adapted with permission from Korennykh et al. 2009) 
at conserved recognition sequences in the pre-mRNA for the transcription factor XBP1 (in metazoans) or HAC1 (in S. cerevisiae) (Chen and Brandizzi 2013). Cleaved exons are then ligated by tRNA ligase in the case of HAC1 (Sidrauski et al. 1996) and RtcB for XBP1 (Lu et al. 2014b) generating the spliced form of HAC1 or XBP1 mRNA (Tam et al. 2014). RIDD leads to decay of mRNAs (Hollien and Weissman 2006; Hollien et al. 2009; Kimmig et al. 2012). XBP1/ HAC1 drives a large transcriptional program to adjust the ER's protein-folding capacity according to the protein folding load in the ER lumen (Cox et al. 1993; Mori et al. 1993; Sidrauski et al. 1996; Yoshida et al. 2001; Calfon et al. 2002). The UPR (unfolded protein response) is activated in cancers, viral infections, as well as neurodegenerative, metabolic, and inflammatory diseases (Naidoo et al. 2005; Atkin et al. 2008; Wang and Kaufman 2016).

IRE1 engaged in RIDD must be coupled with mRNA degradation enzymes in order to prevent either translating or ligating cleavage products (Tam et al. 2014). One report suggested that the two RNase activities, RIDD and splicing, were controlled by different protein oligomeric states, with splicing a result of the filamentous form, and RIDD the dimeric form (Tam et al. 2014). Another suggests that the different outcomes following cleavage by IRE1 (ligation or degradation) have more to do with RNA sequence and structure, and perhaps cellular location of mRNAs (Li et al. 2018). mRNAs that are degraded via RIDD encode proteins to lessen the ER stress burden and in extreme cases initiate apoptosis. During the adaptive response, IRE1 conducts RIDD on mRNAs encoding ER translocating proteins to prevent further increases in protein-folding demand in the ER (Han et al. 2009). In metazoans, RIDD reduces the load of ER client proteins through mRNA degradation and cleavage of $28 \mathrm{~S}$ rRNA (Iwawaki et al. 2001), particularly of mRNA that is associated with the ER membrane (Hollien and Weissman 2006; Han et al. 2009; Hollien et al. 2009; Kimmig et al. 2012; Mishiba et al. 2013). Also in metazoans, it has been shown that $21 \%$ of RIDD substrates encode proteins associated with gene expression regulation that might also participate in the global decrease in protein production (Maurel et al. 2014), and that RIDD reduces the total protein influx into the ER by $15 \%$ (Kimmig et al. 2012).

Mammals have two IRE1 paralogs, IRE1 $\alpha$ and IRE1 $\beta$. IRE $1 \alpha$ is expressed ubiquitously and knockout mice exhibit embryonic lethality. IRE $1 \beta$ expression is restricted, and knockout mice are viable; therefore, the $\alpha$ is thought to be more important (Chen and Brandizzi 2013). The two IRE1 modalities (splicing and RIDD) co-exist in metazoan cells (Hollien and Weissman 2006; Hollien et al. 2009; Moore and Hollien 2015), yet are evolutionarily separated in the two yeast species, $S$. cerevisiae and $S$. pombe. S. cerevisiae IRE1 has splicing, but no RIDD (Niwa et al. 2005; Kimmig et al. 2012), while $S$. pombe IRE1 has RIDD but no splicing
(Kimmig et al. 2012). Mammalian IRE1 $\alpha$ is more efficient in XBP1 mRNA splicing, while IRE1 $\beta$ prefers to cleave (leading to degradation) ribosomal RNA (Iwawaki et al. 2001; Imagawa et al. 2008; Nakamura et al. 2011).

If attempts to restore ER homeostasis fail, IRE1 activates apoptosis through RIDD (Han et al. 2009; Upton et al. 2012), degrading mRNA for UPR target genes and miRNAs that are anti-Casp2 (Chen and Brandizzi 2013). The latter results in upregulation of Casp2, a proapoptotic protease essential for the execution of apoptosis (Upton et al. 2012). Therefore, IRE $1 \alpha$ is a molecular switch and apoptosis executioner during ER stress (Chen and Brandizzi 2013).

X-ray crystal structures of the luminal and cytosolic IRE1 have been reported. Those of the luminal domain show the basis for their signal dependent (i.e., unfolded protein binding) dimerization/oligomerization for $S$. cerevisiae (Credle et al. 2005) and human (Zhou et al. 2006). Structures of the cytosolic domain have been solved in dimeric forms (yeast (Lee et al. 2008) and human (Ali et al. 2011)), as well as a filamentous form of yeast IRE1 (Korennykh et al. 2009). The yeast dimeric structure shows the kinase domain dimerizing backto-back, which places the kinase active sites too far apart for auto-phosphorylation. However, a dimeric structure of the unphosphorylated human IRE1 shows face-to-face orientation of kinase domains, well positioned to autophosphorylate (Ali et al. 2011). The authors interpret this structure as an early state, prior to phosphorylation, afterwhich the dimer assumes the back-to-back orientation that activates the RNase activity. The filament structure shows the $S$. cerevisiae IRE1 cytosolic domains in complex with a kinase inhibitor, which is a potent activator for the IRE1 RNase (Korennykh et al. 2009). The oligomeric structure positions the kinase domains perfectly for autophosphorylation and orders the RNase domain of each IRE1 protomer. The filament creates surface for RNA binding and positioning of the splice sites. The filament is a righthanded helical filament made by the addition of the back-toback dimers, hence a run-on oligomer (Fig. 22f). Each dimer is separated by a $54.2^{\circ}$ rotation, giving 7 dimers per turn. It was found in the structure that 17 residues of the IRE1 cytosolic domain were phosphorylated, which must have occurred via autophosphorylation during expression in E. coli. The three phosphorylated residues important for IRE1 activation in vivo, Ser 840p, Ser 841p, Thr844p, are well resolved in the crystal structure (Korennykh et al. 2009) and are ideally placed to help IRE1 oligomerization (Korennykh et al. 2009).

The trans-autophosphorylation as well as binding to ADP contribute to activation of the RNase of IRE1 (Korennykh et al. 2009). In the oligomeric structure, the RNase domains are ordered, unlike in the dimeric structures, and the ordering appears to be due to intermolecular interactions only found in the oligomer. The ordering of the RNase domain structure is expected to activate its enzymatic activity (Korennykh et al. 2009). Binding of a cofactor occurs in the open state of IRE1 
kinase, shifts the equilibrium from monomers towards multimers and provides an additional, phosphorylation independent level of positive modulation for the activating transition (Korennykh et al. 2009). Oligomerization of the unphosphorylated IRE1 opens the kinase domain and positions it for trans autophosphorylation. ATP enters the opened kinase and phosphorylates the activation loop in trans to lock it in the oligomerization compatible open state and to introduce a phosphate-mediated salt bridge at the interface IF3c (Korennykh et al. 2009). These events provide positive feedback for oligomer assembly (Korennykh et al. 2009). Cofactor binding and phosphorylation enhance the self-association properties of IRE1, but neither is strictly required (Korennykh et al. 2009).

\section{Casein kinase 2}

Casein kinase 2 (CK2) or casein kinase 2 is a highly conserved serine/threonine kinase (Fig. 23a) that is crucial for cell viability and is involved in cellular processes such as cell cycle control, cellular differentiation, and proliferation, circadian rhythm, apoptosis, and gene expression (Litchfield 2003; Meggio and Pinna 2003). Human CK2 is a heterotetrameric holoenzyme $(\alpha 2 / \beta 2)$ composed of two catalytic alpha subunits attached to a central, regulatory dimer of $\beta$ subunits.
CK2 $\alpha$ is constitutively active, and upon interaction with CK2 $\beta$ remains active but its thermostability, substrate specificity, and ability to attach and penetrate cell membranes is altered. Conventional kinase regulatory mechanisms such as phosphorylation, dephosphorylation, or second messenger binding are not observed for CK2 (Poole et al. 2005). Instead, regulation of enzyme activity is proposed to occur by controlling the oligomeric state of the enzyme (Valero et al. 1995). A mixture of oligomeric species including heterotetramers, ring like oligomers, and linear polymers has been observed using electron microscopy, analytical ultracentrifugation, and native mass spectrometry (Fig. 23b, c) and found to vary as a function of ionic strength, polycations, $\mathrm{Mg}^{2+}$ concentration, $\mathrm{pH}$, and temperature (Glover 1986; Mamrack 1989; Valero et al. 1995; Seetoh et al. 2016). Enzyme activity is maximal under conditions favoring the ring-like structures and decreases with dissociation into the heterotetramers or association into filaments (Valero et al. 1995). CK $2 \alpha$ without CK2 $\beta$ is constitutively active and does not form higher assemblies (Valero et al. 1995). Crystal structures of CK2 show a circular trimeric oligomer (1JWH (Niefind et al. 2001), and 4DGL (Lolli et al. 2014)) or linear polymers (4MD7-4MD9 and 4NH1 (Schnitzler et al. 2014)). Native mass spectrometry using ion-mobility mass spec and $\mathrm{H} / \mathrm{D}$ exchange confirmed the mixture of oligomeric states
Fig. 23 Reaction catalyzed and structures formed by casein kinase 2 (CK2). a Protein kinase activity of CK2. b Negative stain EM of CK2 showing different oligomeric and filamentous forms (adapted with permission from Valero et al. 1995). Scale bar corresponds to $100 \mathrm{~nm}$. c Schematic of interconversion of oligomeric forms of $\mathrm{CK} 2$ and their dependence on ionic strength (adapted with permission from Seetoh et al. 2016)
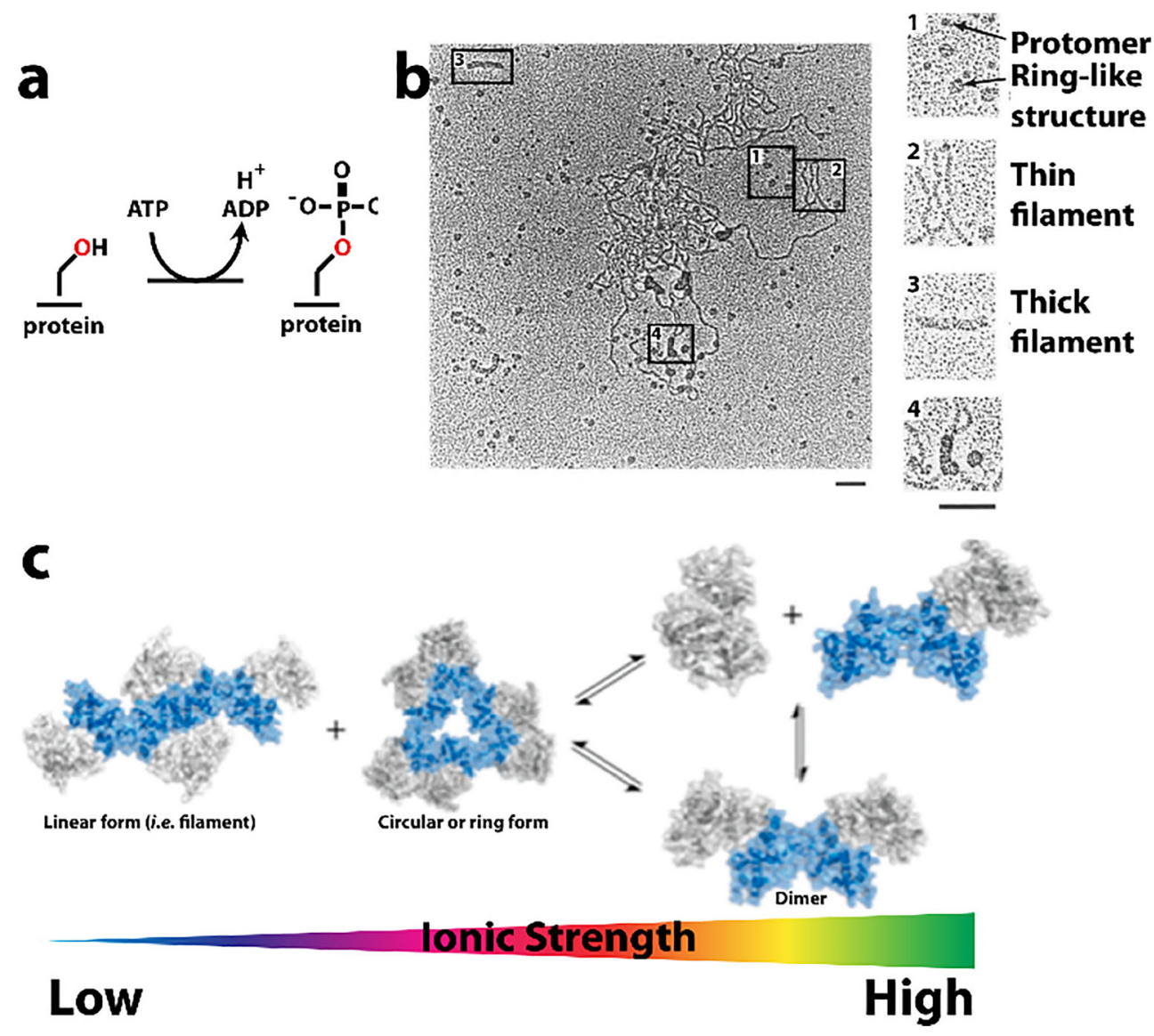
including heterotetrameric protomers, tetrameric ring like structures, and linear polymers which are sensitive to ionic strength (with high ionic strength causing disruption of the higher order forms) (Seetoh et al. 2016). The stoichiometry of the complex also changes with ionic strength $\left(\alpha_{1}, \beta_{2}\right)_{1}$ to $\left(\alpha_{2}, \beta_{2}\right)_{1}$ ratio increases with decreasing ammonium acetate concentration (Seetoh et al. 2016). Formation of oligomers and aggregates was shown in cells using BRET (bioluminescent resonance energy transfer) and found to be non-static (Hubner et al. 2014). The CK2 binding protein alpha subunit of the heterotrimeric G-protein that stimulates adenylyl cyclase (Gas), as well as the polycationic compound polylysine. Gas, but not the CK2 substrate $\beta$-arrestin2, reduced the BRET signal associated with aggregation of CK2 (Hubner et al. 2014).

\section{Adenylate kinase}

Adenylate kinase (AK) performs the reaction shown in Fig. 24a and is found in plants and bacteria. Wild and coworkers (Wild et al. 1997) determined the crystal structure of AK from maize with an inhibitor (adenosine-(5')pentaphospho( $5^{\prime}$ )adenosine (Ap5A) and found that the $24.9 \mathrm{kDa}$ enzyme crystallized in a manner that produced infinite, linear "proto-rods" composed of hexamers (Fig. 24b-d). Based on contact surface area arguments, the authors propose that this is more than merely crystallographic packing. The authors also argue that the enzyme must be inactive in this linear assembly due to closing of a "lid" domain that cuts off access to the active site, and this "lid" domain participates in close contacts within the rod. The function of the rod, proposed by Wild and coworkers is to store AK during nighttime, when photosynthesis is low and AK activity is not required (Wild et al. 1997). Water would also be released from the rod, which is required during the night for plants. Once daytime arrives, the AK enzymes may quickly disassemble and become active again, avoiding the need for synthesis of new enzymes. However, no further enzymological studies of the formation of rods in vivo, or the effect of rod formation on enzyme activity have been reported.

\section{Receptor-interacting serine/threonine-protein kinases (RIP1/RIP3 kinases)}

RIP1 and RIP3 are intracellular signaling Ser/Thr kinases (Fig. 25a) that play important roles in immune defense, cancer, and neurodegenerative diseases. RIP1 controls whether the pleiotropic cytokine TNF $\alpha$ induces NF-kB activation, apoptosis, or programmed necrosis (Moquin and Chan 2010). Programmed necrosis involves formation of an assembly, the necrosome, with RIP3 with activated kinase activity (Cho et al. 2009; He et al. 2009a; Zhang et al. 2009) (Fig. 25b). The necrosome recruits MLKL (Mixed Kinase Domain Like

\section{a \\ $A T P+A M P \rightleftharpoons 2$ ADP}
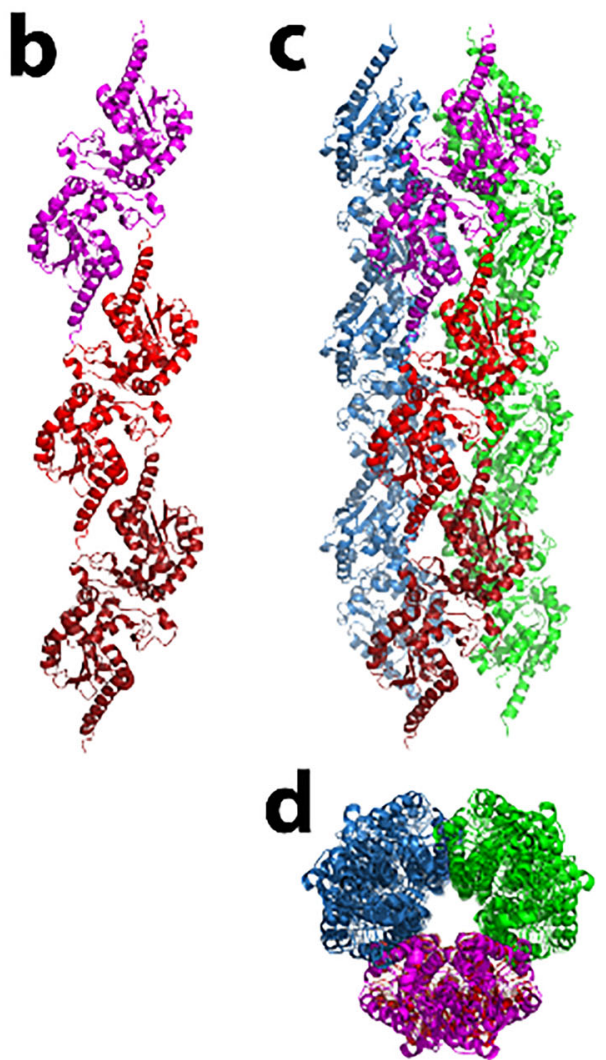

Fig. 24 a Reaction catalyzed by maize adenylate kinase (EC 2.7.4.3). bd Crystallographic packing in crystals of AK from maize (PDB code 1ZAK) resembling a single infinite filament, three protomers shown and colored distinctly (b), three filaments assembled around a crystallographic 3-fold (c), and viewed from the top (d)

protein), which is phosphorylated by RIPK3 and immediately translocates to lipid rafts inside the plasma membrane (Cho et al. 2009; Sun et al. 2012; Zhao et al. 2012). This leads to the formation of pores in the membrane, allowing the sodium influx to increase and consequently also the osmotic pressure, which eventually causes cell membrane rupture (Cho et al. 2009). Phosphorylation stabilizes the enzyme assembly, which is mainly formed via interactions between a short segment (5-6 residues) of each protein known as the RIP homotypic interaction motif (RHIM) (Li et al. 2012a) (brown rectangle in Fig. 25b). This motif is surrounded by unstructured regions but forms a cross-beta amyloid structure through self-association or association of RIP1 and RIP3 with each other ( $\mathrm{Li}$ et al. 2012a) (Fig. 25c). This association is critical for function of RIP1 and RIP3 kinases in programmed necrosis ( $\mathrm{Li}$ et al. 2012a). Amyloid is distinct in its structure and binding properties, with this amyloid shown to be 


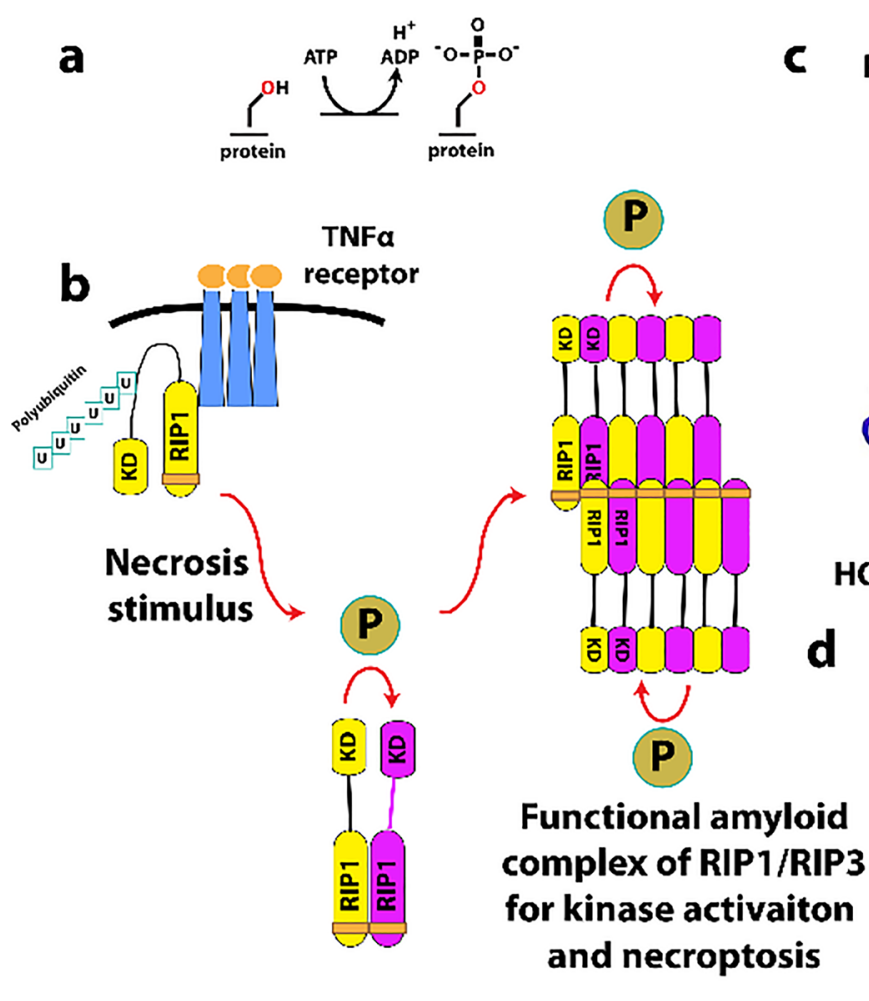

Fig. 25 Reactions catalyzed by and structures formed by RIP1/RIP3 kinases. a Protein phosphorylation reaction catalyzed by the kinase domain of RIP1 and RIP3. b Formation of amyloid via the RHIM domain (brown) activates its kinase activity (adapted with permission from $\mathrm{Li}$ et al. 2012a). c Proposed structure of the RHIM or amyloid forming

composed of alternately stacked RIP1 and RIP3 RHIMS to form heterotypic beta sheets. Two such beta sheets bind together along a compact hydrophobic interface featuring an unusual ladder of alternating Ser (from RIP1) and Cys (from RIP3) residues (Mompean et al. 2018). EM shows filaments of RIP1/RIP3 with its globular domains (kinase on both and death domain on RIP1) extending outward from a core protease resistant amyloid filament, and these structures appear to also interact to form larger aggregates ( $\mathrm{Li}$ et al. 2012a) (Fig. $25 \mathrm{~d}$ shows a filament of RIP1 and the RHIM of RIP3). These are also seen from RIP1/RIP3 complexes purified from cells undergoing programmed necrosis, but not controls, where foci are seen (Fig. 25e). The amyloid is remarkably resistant to denaturation, requiring $150 \mathrm{mM} \mathrm{NaOH}$ to disrupt ( $\mathrm{Li}$ et al. 2012a). The RHIM, which mediates amyloid formation, is found in a growing number of signaling adaptors with crucial functions in cell death and innate immunity (Moquin and Chan 2010). Amyloids are fibrous protein aggregates composed of cross-beta structures and associated with many neurodegenerative (Chiti and Dobson 2006) and infective prion diseases (Uptain and Lindquist 2002). Amyloids can perform normal cellular functions, such as host interaction, hazard protection, and memory storage (Chiti and Dobson 2006). The purpose of amyloid/fibril formation may be for feed-forward
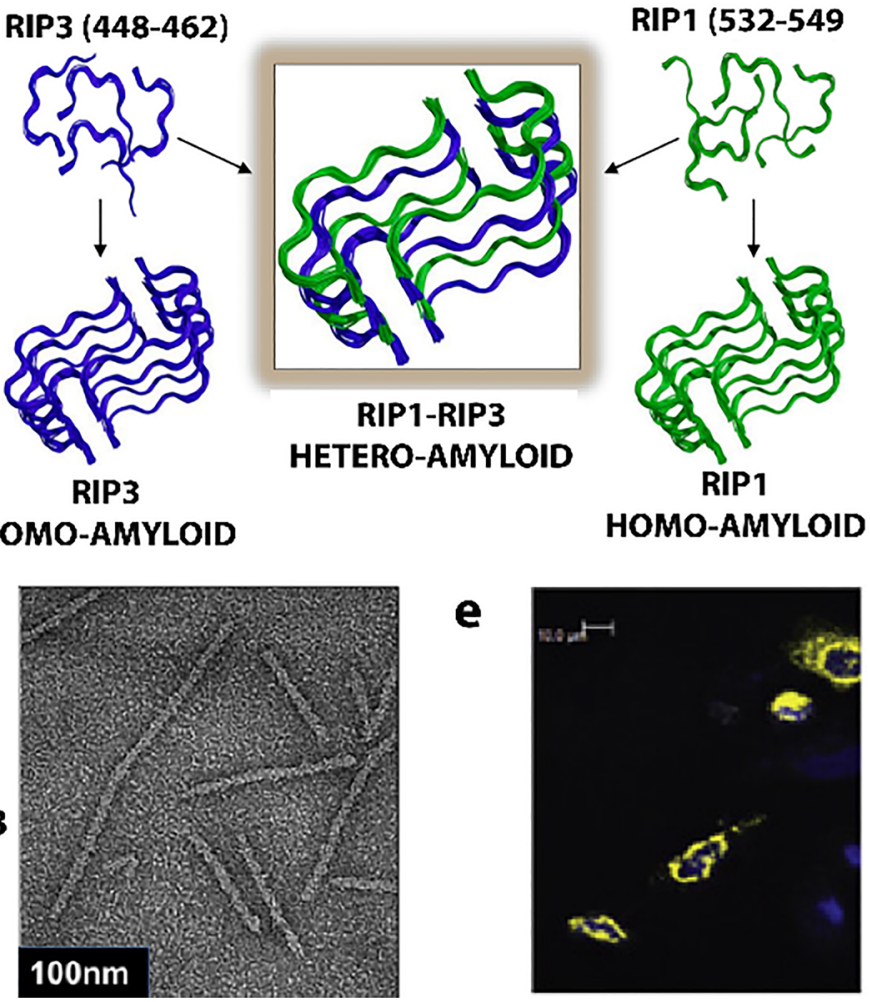

e

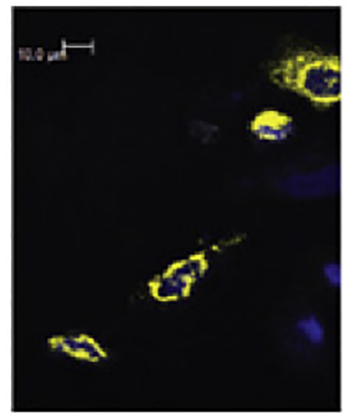

domains of RIP1 and RIP3 kinases (adapted with permission from Mompean et al. 2018). d Negative stain EM of filaments formed by RIP1 and the RHIM sequence of RIP3 (adapted with permission from Li et al. 2012a). e Foci formed in HeLa cells of YFP labeled RIP3 (adapted with permission from Li et al. 2012a)

gain of function in which kinase activation and RIP1/RIP3 necrosome formation are mutually reinforcing $(\mathrm{Li}$ et al. 2012a). Upon amyloid formation, transphosphorylation occurs which activates RIP1/RIP3 (Li et al. 2012a).

\section{Death domain containing proteins that form filaments}

Filament-forming proteins are increasingly recognized as critical intermediaries in signaling pathways including those of innate immunity, necrosis, and apoptosis (Ferrao and $\mathrm{Wu}$ 2012; Yin et al. 2015). These pathways contain proteins which upon initial stimulation (such as binding to a pathogen associated molecule in the case of innate immunity) leads to filament nucleation, propagation, and recruitment of downstream effectors such as kinases, transcription factors, caspases, and interleukins. The subcellular structures involved in innate immunity are known as inflammasomes. Filaments are formed similarly, via the Death Domain (CARD, PYD, DED). Example proteins include RIG-I, MDA5, and MAVS) (Zeng et al. 2010; Wu et al. 2014), NOD1/NOD2 and RIP2 (Gong et al. 2018), TLRs, MyD88, IRAK2, and IRAK4 (Lin et al. 2010), NLRPs, ASC, and Caspase-1 (Lu et al. 2014a; Diebolder et al. 2015; Lu et al. 2016), and Fas-FADD 
(Wang et al. 2012). Not all are enzymes, but many contain protein kinase domains. Figure 26a shows foci formed by GFP labeled RIP2, a CARD domain containing kinase that functions in innate immunity. Figure $26 \mathrm{~b}$ shows a negative stain EM image of RIP2-CARD. Figure 26c shows a model from a three-dimensional reconstruction of a filament composed of the PYD domain of ASC. Though the different filaments formed by death domain containing proteins share many common features, studies show that they can have different geometries, including different handedness (Diebolder et al. 2015; Lu and Wu 2015). Figure 26d gives models for inflammasome assembly of two different innate immune pathways that involve filament formation by DD containing proteins.

\section{Target of rapamycin complex 1 (TORC1)}

TOR is a eukaryotic serine/threonine kinase found in TORC1 and TORC2, and TORC1 has been found to form foci in cells (Fig. 27a) composed of hollow, helical, cylindrical assemblies
(Fig. 27b-c) upon glucose starvation (Prouteau et al. 2017). The kinase is inactive in this assembly, which forms at vacuoles, and is dependent on Rag GTPases for its assembly through some unknown process (Prouteau et al. 2017). Prouteau and colleagues suggest that this assembly, they call the TOROID, may have a function to inactivate an expensive multiprotein complex in a cost-effective way (Prouteau et al. 2017). TORC1 functions as a nutrient/energy/redox sensor and controls protein synthesis.

\section{Enzymes shown to form foci in cells but assembly architecture not known}

\section{Glutamate synthase (Glt1 in yeast)}

Glutamate synthase forms L-glutamate from L-glutamine and 2-oxoglutarate which is an alternative pathway to L-glutamate dehydrogenase for the biosynthesis of L-glutamate (Fig. 28a), and participates with glutamine synthetase in ammonia a

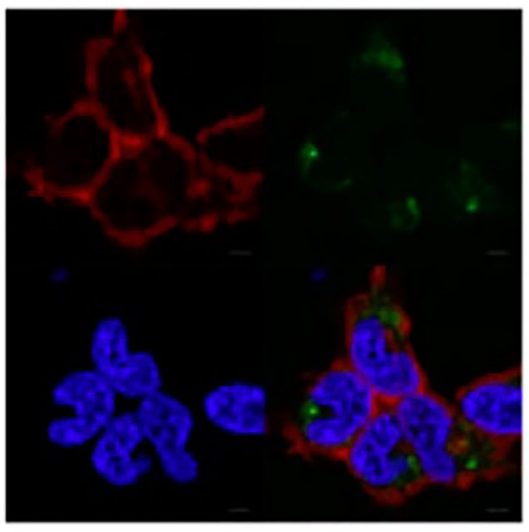

C
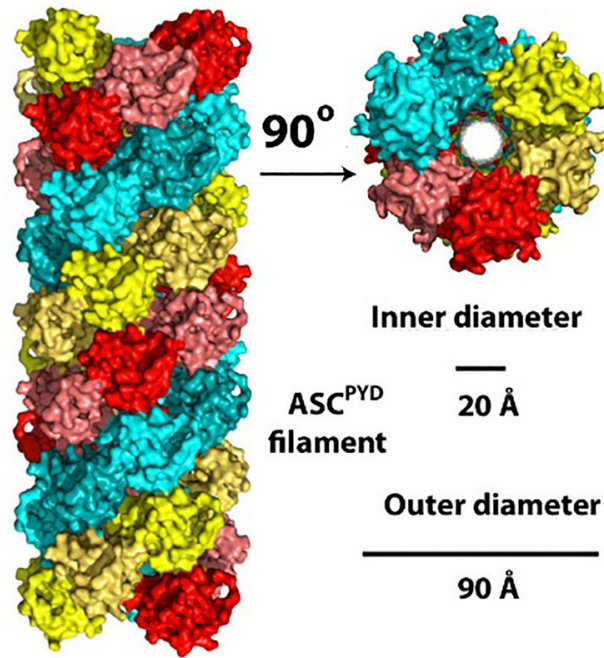

Inner diameter

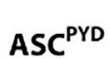

$20 \AA ̊$

filament

Outer diameter

$90 \AA ̊$

\section{RIP2wt-full

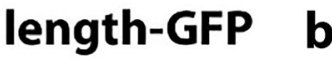

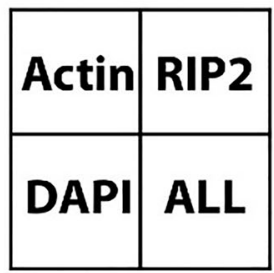

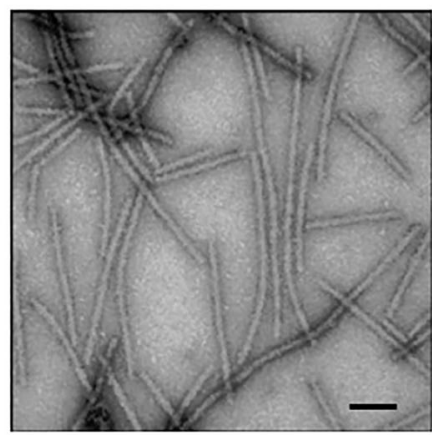

d
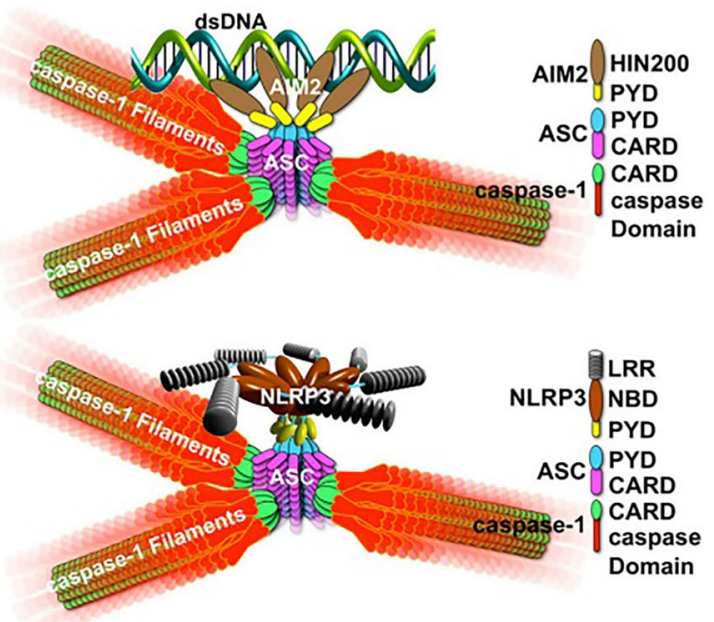

can be thought of as composed of 3 single strands in a right-handed helix (adapted with permission from Lu et al. 2014a). d Models of inflammasome signaling involving protein filamentation (adapted with permission from Lu et al. 2014a) 
a

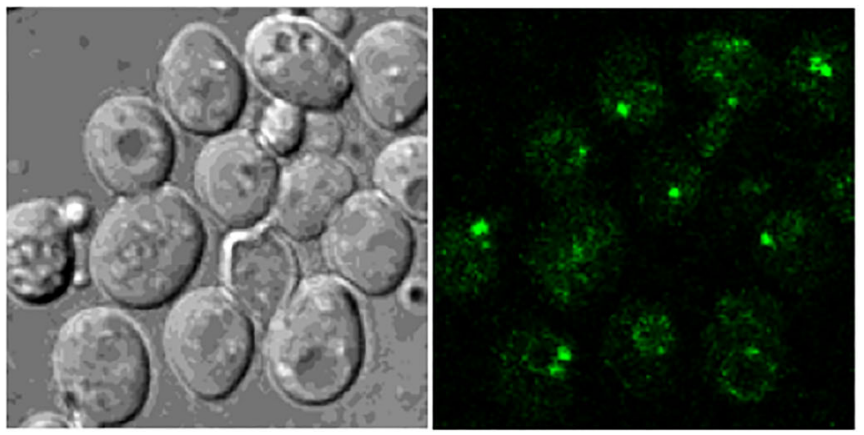

b

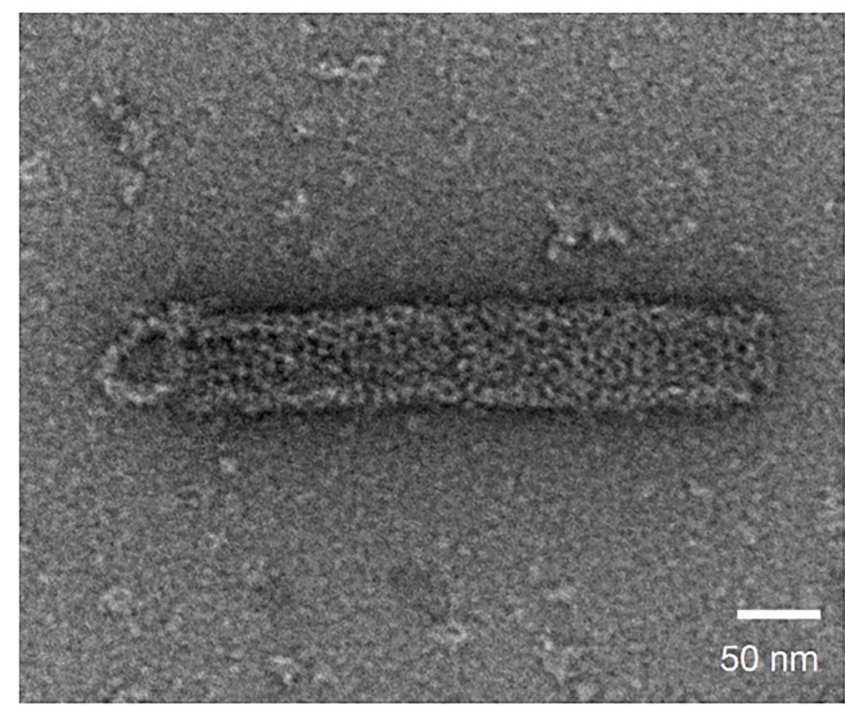

C

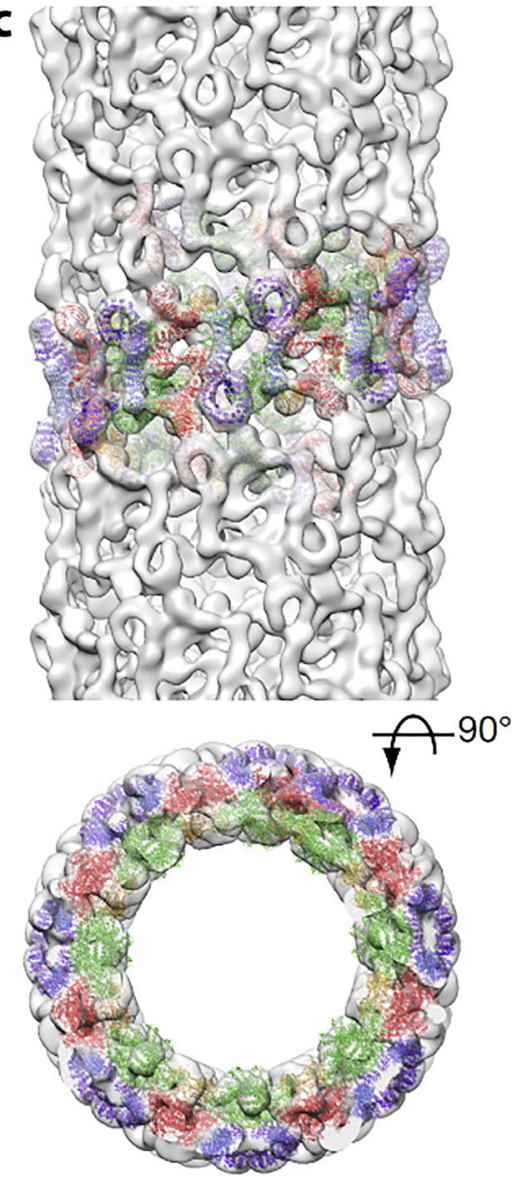

Fig. 27 Filament formation by TORC1. a Foci formed by GFP labeled TORC1 (GFP-Kog1) under glucose starvation. Left, DIC image; right, confocal image (adapted with permission from Prouteau et al. 2017). b Negative stain EM micrograph of TORC1 filament (adapted with permission from Prouteau et al. 2017). c Single particle reconstruction at $27 \AA$ resolution of TORC1 filament with constituent coordinate files fit into density (adapted with permission from Prouteau et al. 2017) assimilation processes. The enzyme is specific for NADH, Lglutamine and 2-oxoglutarate. Both Noree and coworkers (Fig. 28b) and Shen and coworkers found that this enzyme forms foci and large rod-like structures (i.e., cytoophidia) in yeast cells when attached to GFP (or an HA tag) (Noree et al. 2010; Shen et al. 2016)). Noree and coworkers performed a number of additional tests investigating the control of selfassembly formation, such as measuring the self-assemblies at different phases of cell growth (log-phase, diauxic, and saturation) and found similar self-assembly in all (Noree et al. 2010). Shen and coworkers found that self-assembly formation increased in diauxic and saturation in both quantity and length (Shen et al. 2016). Noree and coworkers found that the Glt1p self-assemblies did not colocalize with other selfassembly forming enzymes (Ura7p or Ura8p (both CTPS), Psaip (GDP-mannose pyrophosphorylase), Gcd2p (eIF2b-delta), Gcd6p (eIF2b- $\varepsilon$ ), Gcd7pg (eIF2B- $\beta$ ), Gcn3p (eIF2b- $\alpha$ ) or Sui2p (eIF2- $\alpha)$ )(Shen et al. 2016). Gltp1 self-assembly formation was also unaffected by known regulators of prion biogenesis (RNQ1 and HSP104), nutrient deprivation, carbon source depletion, energy status of the cell (by addition of sodium azide), protein synthesis (by addition of cycloheximide), or low temperature (Noree et al. 2010).

Shen and coworkers measured dynamics of Glt1p selfassembly formation and found that these structures are motile and located preferentially at the cell cortex (Shen et al. 2016). Some are highly dynamic, while others are relatively slow. Analysis of the dynamics indicates that the self-assemblies undergo sub-diffusion, i.e., random diffusion within confined areas that is not driven by motor proteins.

\section{Asparagine synthetase}

Asparagine synthetase (paralogous yeast genes Asn1 and Asn2) catalyzes the synthesis of L-asparagine from Laspartate and L-glutamine in an ATP-dependent reaction and is part of the asparagine biosynthetic pathway) (Fig. 28c). Shen and coworkers uncovered rod shaped self-assemblies formed by this enzyme (Fig. 28d), and that these selfassemblies grew on going from log-phase growth to diauxic and stationary (Shen et al. 2016). These enzymes can form 
a

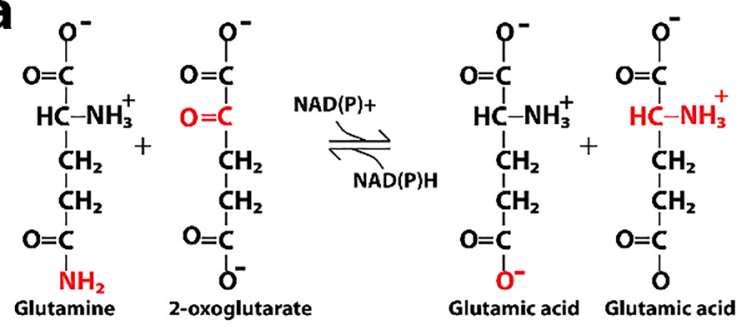

b

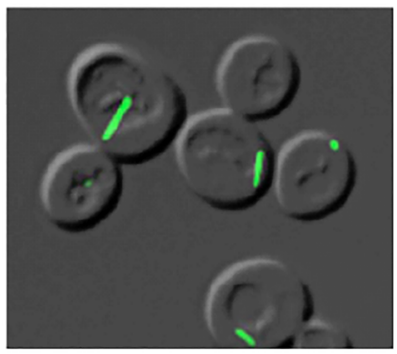

e

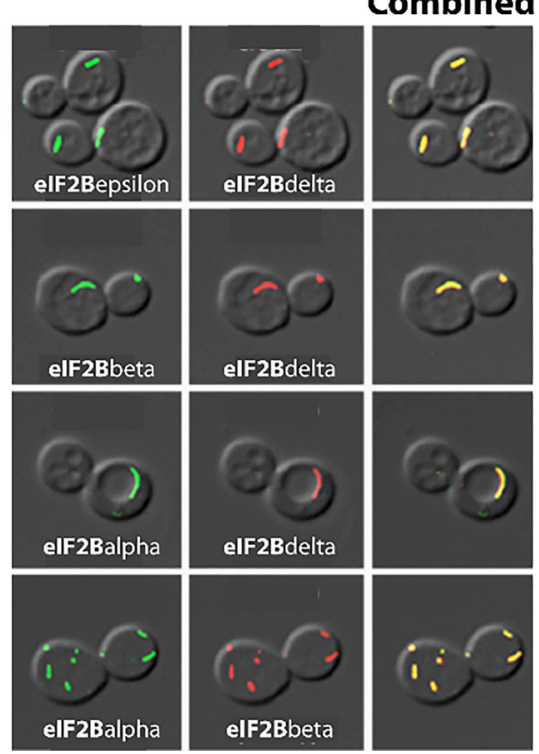

Fig. 28 a Reaction catalyzed by glutamate synthase (Glt1 in yeast) (EC 1.4.1.13). b Cytoophidia formed by GFP labeled Glt1p in $S$. cerevisiae (adapted with permission from Noree et al. 2010). c Reaction catalyzed by asparagine synthetase (EC 6.3.5.4). d Cytoophidia formed by GFP labeled asparagine synthetase (adapted with permission from Shen et al. 2016). e Colocalization of various eIF2 and eIF2B proteins in

self-assemblies in both the cytoplasm and nucleus (Shen et al. 2016).

Zhang and coworkers also studied the self-assembly formation of asparagine synthetase in yeast and found an increase in self-assembly formation with glucose deprivation (Zhang et al. 2018). It has also been shown that colocalization of the two paralogs occurs, and that self-assembly formation of Asn2 is dependent on Asn1 (Zhang et al. 2018; Noree et al. 2019b). Although forming distinct structures (i.e., cytoophidia), both asparagine synthetase and CTPS cytoophidia are found adjacent to one another in cells, suggesting a connection, possibly metabolic.
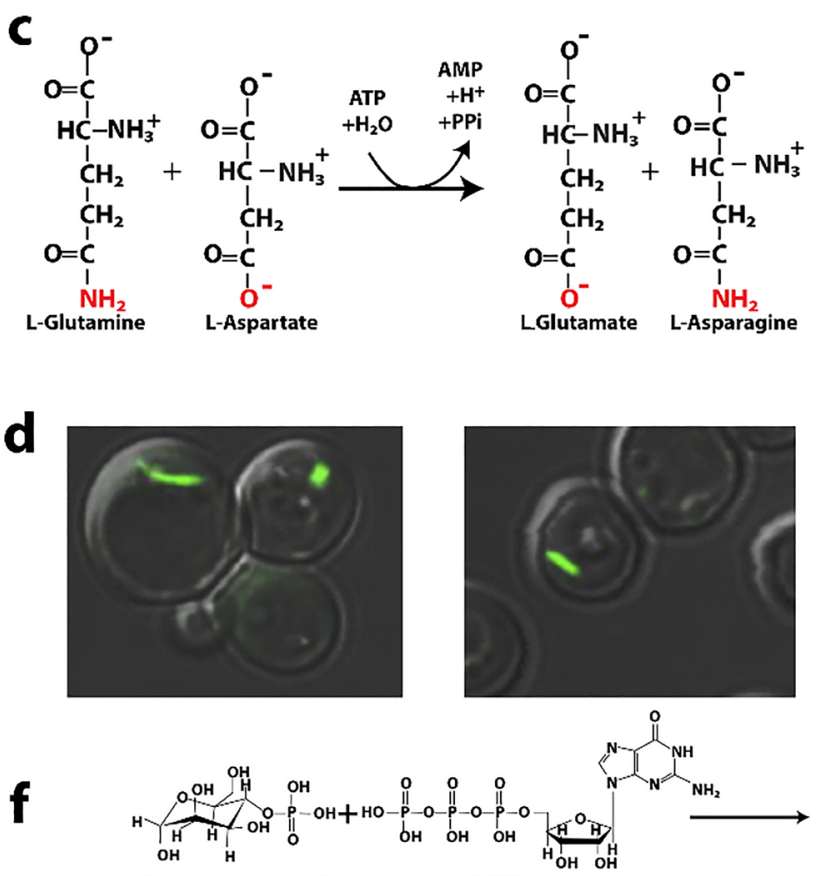

Manose-1-P

GTP
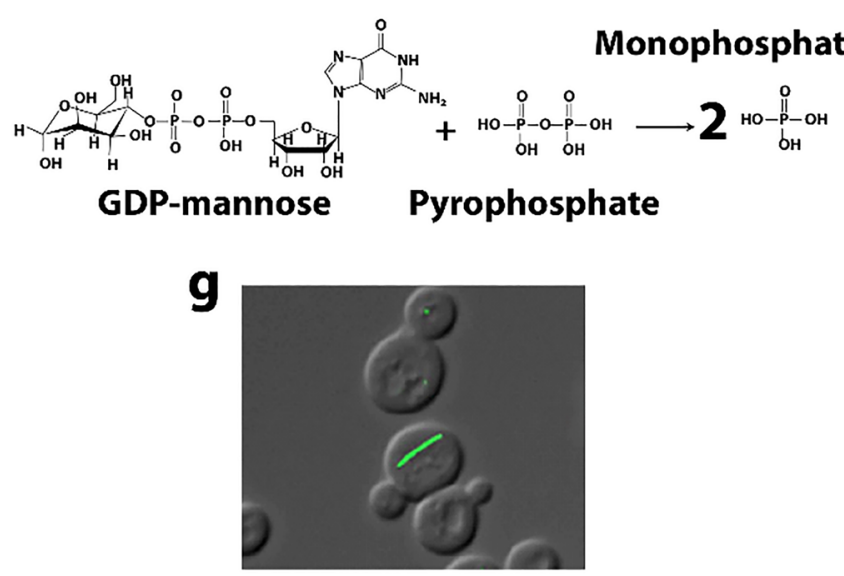

cytoophidia, and lack of colocalization with other, unrelated proteins (adapted with permission from Noree et al. 2010). f Reaction catalyzed by GDP-mannose pyrophosphorylase (EC 2.7.7.13). g Cytoophidia formed by GFP labeled GDP-mannose pyrophosphorylase (adapted with permission from Noree et al. 2010)

\section{Eukaryotic translational initiation factor (elF2/2B)}

Noree and coworkers found proteins of the eIF2/eIF2B genes (Gcd2 (eIF2B- $\delta$ ), Gcd7 (eIF2B- $\beta$ ), Gcd6 (eIF2B- $\varepsilon$ ), and Sui2 $(\mathrm{eIF} 2-\alpha), \mathrm{Gcn} 3(\mathrm{eIF} 2 \mathrm{~B}-\alpha))$ formed cellular self-assemblies resembling rods (with either GFP or HA tags) (Noree et al. 2010). These authors also tested for colocalization, using representative subunits of eIF 2 and eIF2B $(\mathrm{Gcd} 2$ or eIF2B- $\delta$ and Gcd7 or eIF2B- $\beta$ ) and found that these do not colocalize with other self-assembly forming proteins (CTP synthase, glutamate synthase, GDP-mannose pyrophosphorylase) but do 
colocalize with each other (and Gcd6/eIF2B-e) (Fig. 28e). They found that eIF2/2B self-assemblies are not affected by known regulators of prion biogenesis (RNQ1 or HSP104), declined somewhat in saturated cultures (although varied by type of subunit), were not affected by carbon source depletion, or sodium azide (affecting energy status of the cell), were affected by an inhibitor of protein synthesis (cycloheximide) (see also (Campbell et al. 2005)), and were not affected by lowering the temperature to $0{ }^{\circ} \mathrm{C}$ or addition of the kinase inhibitor staurosporine. Shen and coworkers confirmed the findings of Noree and coworkers, and discovered selfassembly formation by an additional eIF2B subunit, Gcd2 (eIF2B- $\delta$ ) (Noree et al. 2010, Shen et al. 2016). The eukaryotic translation initiation factor eIF2 mediates the first step of translation, by binding to the initiator met-tRNA and GTP and recognizing the start codon in an mRNA (Dever et al. 1995). GTP is hydrolyzed by eIF2 to GDP after association with the ribosome, which in turn initiates translation. Regeneration of the active, GTP bound form of eIF2 is performed by the nucleotide exchange factor (GEF) eIF2B. Therefore, selfassembly formation by eIF2/eIF2B prevents it from doing its job, no doubt, which is initiation of translation. Confirming this, Nuske and coworkers found that starvation induced acidification of the cytosol causes self-assembly of eIF2B $(\mathrm{Gcn} 3 / \mathrm{eIF} 2 \mathrm{~B}-\alpha)$ and downregulation of translation that is independent of the kinase Gen2 (Nuske et al. 2018).

\section{GDP-mannose pyrophosphorylase (Psa1)}

The yeast gene Psa1, or GDP-mannose pyrophosphroylase (reaction catalyzed by this enzyme shown in Fig. 28f) was found in screens to form self-assemblies in yeast cells during stationary phase (i.e., nutrient starvation) (Fig. 28g) (Noree et al. 2010, Shen et al. 2016). Noree and coworkers performed several additional tests and found that these self-assemblies were distinct from the others tested (Ura7p, Glt1p, and eIF2/2B) (Noree et al. 2010). Like Glt1p self-assemblies, the Psalp self-assemblies were not affected by knockouts of prion formation requiring proteins RNQ1 and HSP104, nor were they affected by overexpression of HSP104. Self-assemblies of Psalp were significantly increased as nutrient starvation increased. Carbon source depletion had no effect on Psalp self-assemblies. They were induced by treatment with sodium azide (affecting energy status of the cell). Cycloheximide or reduction in temperature to $0{ }^{\circ} \mathrm{C}$ had no effect, but addition of the kinase inhibitor staurosporine greatly increased the number of cells with self-assemblies.

\section{Xanthene oxidase}

Xanthene oxidase from mammals is important in purine catabolism and nitrogen metabolism (Fig. 29a). A report in 1987 (Angermuller et al. (1987)) indicated that self-assemblies form of homotetrameric rings of this enzyme form in vitro. No studies have been reported of the effect on enzyme function, or formation of such structures in vivo.

\section{Purinosome}

Enzymes comprising the "purinosome" are responsible for de novo synthesis of purines (Fig. 29b) and include phosphoribosyl-pyrophosphate amidotransferase (PRPPAT, step 1, Fig. 29b), trifunctional GART enzyme (GAR Tfase, steps $2-3$ and step 5, Fig. 29b), FGAMS/PFAS enzyme (step 4, Fig. 29b), bifunctional PAICS enzyme (steps 6-7, Fig. 29b), ADSL enzyme (steps 8, Fig. 29b), and bifunctional ATIC enzyme (steps 9-10, Fig. 29b). These have been shown to form reversible cytoplasmic foci under purine starvation (HeLa cells (An et al. 2008), Fig. 29c) and general nutrient starvation (yeast (Narayanaswamy et al. 2009, Deng et al. 2012)) as well as in the female germ line in Drosophila (Lowe et al. 2014). Narayanaswamy and coworkers showed that the foci formation by Ade4 (i.e., (PRPPAT)) was stimulated by the absence of adenine in growth media and could be reversed upon the addition of adenine (Narayanaswamy et al. 2009). Ade4 punctate body formation was inhibited by the addition of the protein translation inhibitor cycloheximide. These Ade4 foci did not colocalize with P-body marker proteins and only in a limited way with actin bodies.

\section{Pyruvate kinase}

Pyruvate kinase in yeast (gene Cdc19) is involved in carbohydrate metabolism (Fig. 30a) and found to form reversible punctate foci in yeast under starvation conditions (Narayanaswamy et al. 2009), as well as glucose starvation, heat shock, and hypoxia (Jin et al. 2017; Saad et al. 2017). Pyruvate kinase also colocalizes with phosphofructokinase-1 (PFK) and fructose-bisphosphate aldolase in the selfassemblies also called G-bodies (Jin et al. 2017). Formation of the large assemblies is postulated to depend upon a low compositional complexity region (LCR) within Cdc19, as they are prevented by tetramer formation, which sequesters the LCR (Saad et al. 2017). The tetrameric form is favored by binding to fructose 1,6-bisphosphate. Cdc19 aggregation appears to trigger its localization to stress granules and modulates their formation and dissolution. Reversible aggregation is thought to protect $\mathrm{Cdc19}$ from stress-induced degradation, thereby allowing cell cycle restart after stress (Saad et al. 2017). Addition of glucose causes rapid dissolution of the aggregates and did not depend on protein synthesis (i.e., cycloheximide had no effect). The assemblies were also unaffected by inhibitors of the proteasome or vacuolar protein degradation. 
a<smiles>O=C1NC2NC=NC2C(=O)NC(=O)C2=C(Nc3[nH]c(=O)[nH]c(=O)c3N2)OCOO1</smiles>

b

Xanthine

Uric acid
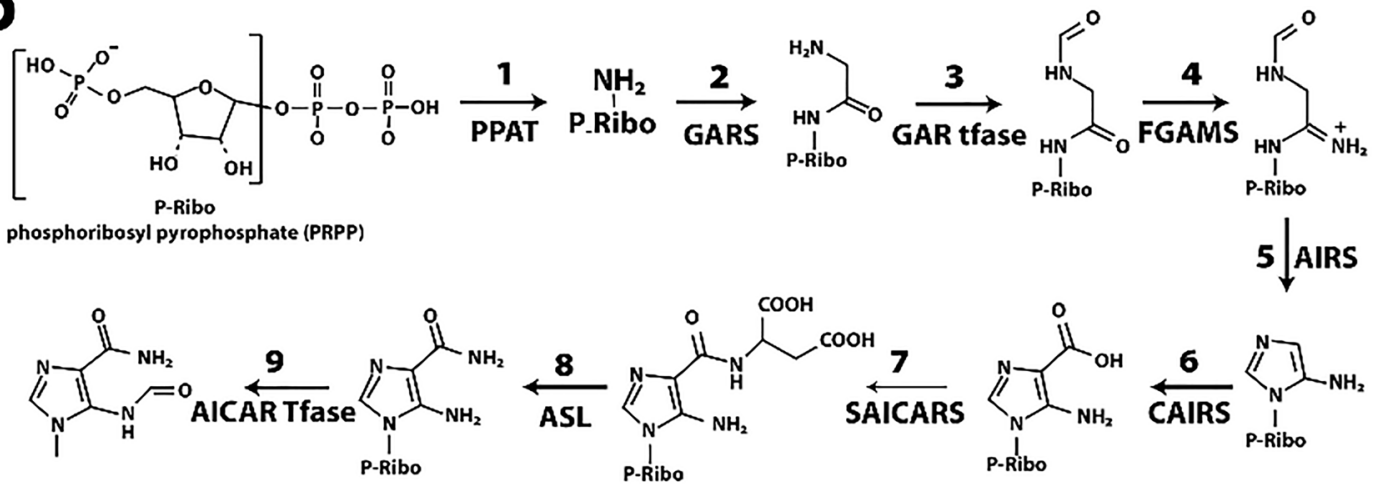<smiles>O=c1[nH]cnc2c1ncn2C1O[C@H](COP(=O)([O-])O)[C@@H](O)C1O</smiles>

C
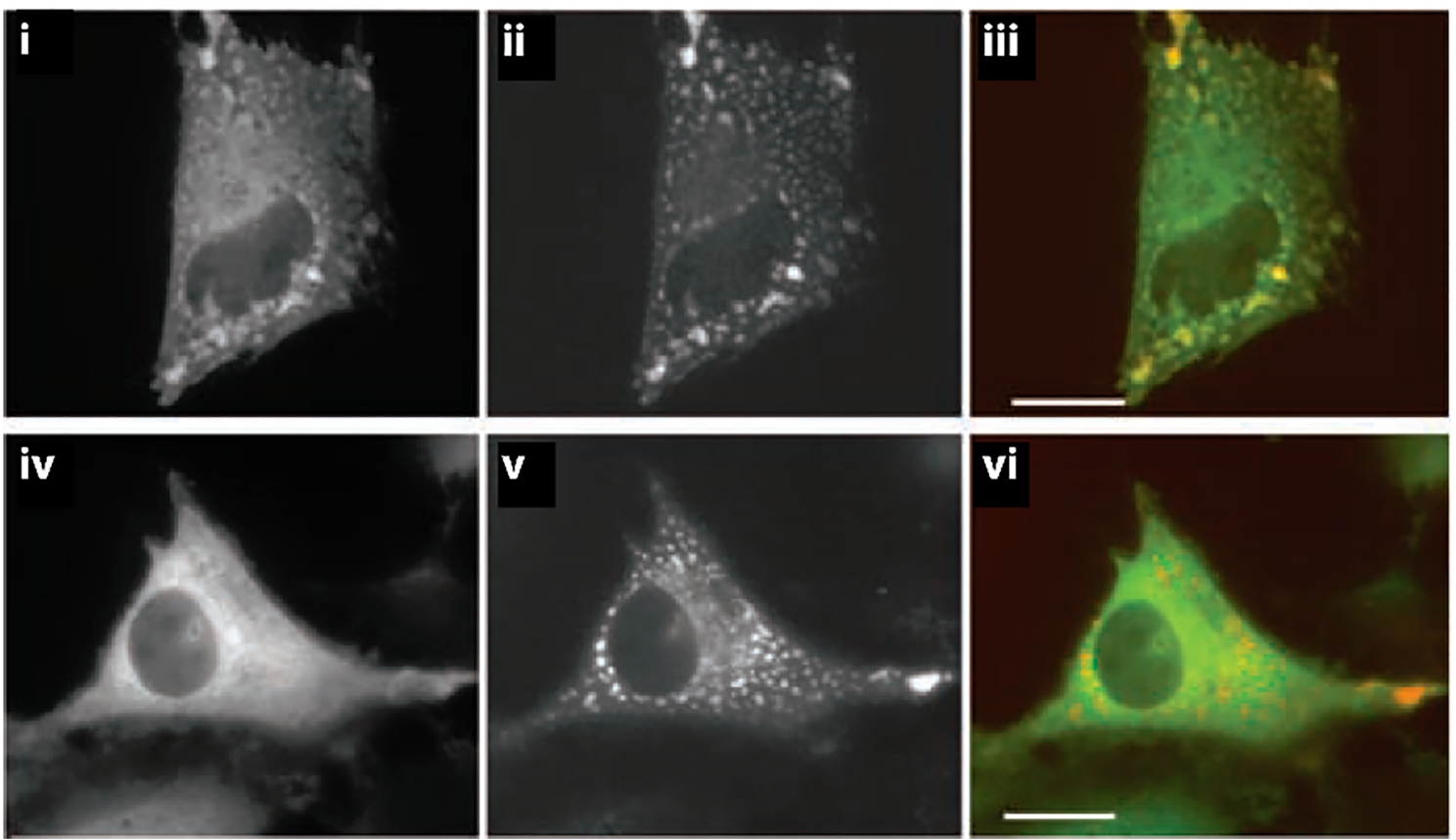

Fig. 29 a Reaction catalyzed by xanthene oxidase (EC 1.1.3.22). b Reactions catalyzed by enzymes of the purinosome (adapted with permission from An et al. 2008). c Foci formed by members of the purinosome in purine depleted media. (i and iii) hTrifGART-GFP [(i) and green in (iii)] coclusters with hFGAMS-OFP [(ii) and red in (vi)] in HeLa cells under purine depleted conditions. (iv to vi) The control
hC1THF-GFP [(iv) and green in (vi)], which does not participate directly in de novo purine biosynthesis, does not form clusters or colocalize with hFGAMS OFP [(v) and red in (vi)] in HeLa cells grown in purinedepleted media. Scale bar, $10 \mathrm{~mm}$ (adapted with permission from An et al. 2008) 
a<smiles></smiles>

Phosphoenolpyruvate

b

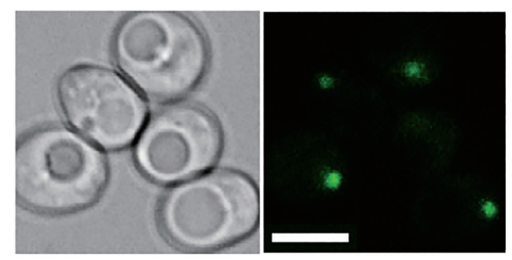

f

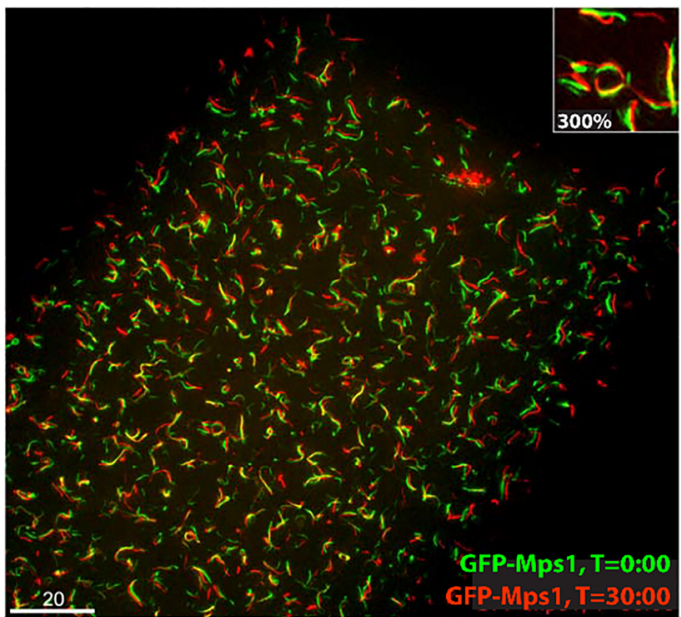

C<smiles>O=CC(O)CO[18O]</smiles>

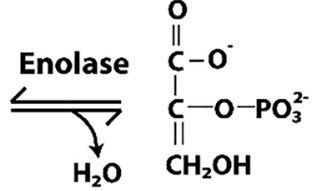

d<smiles>O=C(CO)CO[Na]</smiles>

Dihydroxyacetone phosphate

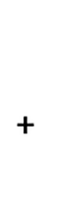

Glyceraldehyde 3-phosphate
Aldolase

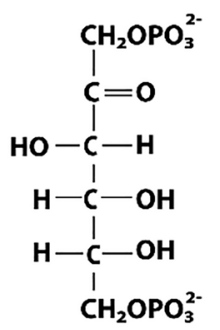

Fructose 1,6bisphosphate

Aldolase-GFP
GFP $\quad$ DIC
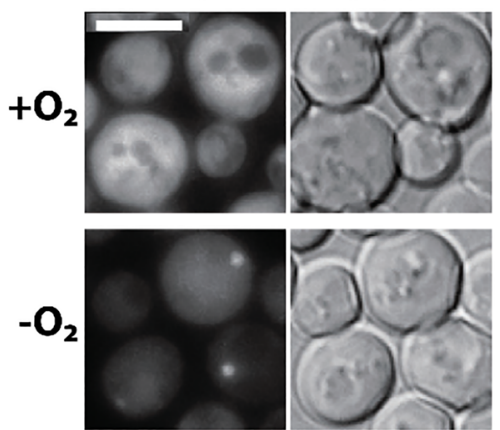

\section{Enolase-GFP}

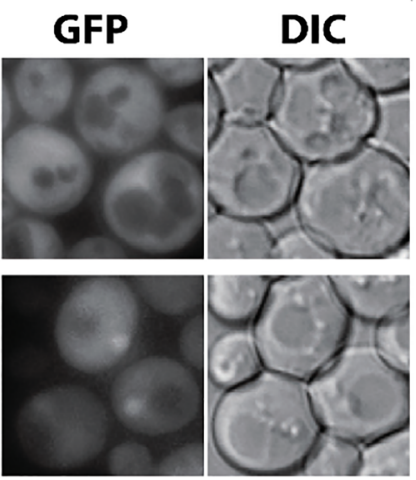

g

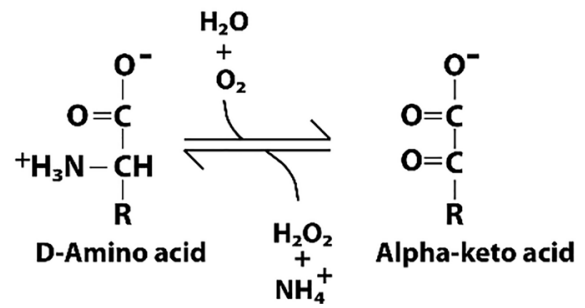

Fig. 30 a Reaction catalyzed by pyruvate kinase (EC 2.7.1.40). b Large assembly formed by GFP labeled Cdc19 in yeast cells under hypoxic conditions (adapted with permission from Jin et al. 2017). c Reaction catalyzed by enolase (EC 4.2.1.11). d Reaction catalyzed by fructose bisphosphate aldolase (EC 4.1.2.13). e G bodies (assemblies) of enolase and fructose bisphosphate aldolase in yeast under hypoxic conditions (adapted with permission from Jin et al. 2017). f Assemblies of Mps1 kinase in Drosophila a single oocyte. Time $=0$ (green) and time $=30$

\section{G-bodies}

Enolas, (yeast-gene Eno2) (Fig. 30c, e) and fructose bisphosphate aldolase (yeast gene Fba1) (Fig. 30d, e), as well as phosphofructokinase (yeast genes Pfk1 and Pfk2) and pyruvate kinase (see above), all part of carbohydrate metabolism, form assemblies known as G-bodies under hypoxic conditions (Jin et al. 2017). Yeast G-bodies have also been shown to be composed of other glycolytic enzymes including phosphoglucose isomerase (yeast gene Pgi1), glyceraldehyde-3-phosphate dehydrogenase (GAPDH) (yeast minutes (red) show assemblies with exposure to $\mathrm{CO}_{2}$ (time $=0$, green), followed by exposure to ambient air whereupon the assemblies dissociate, then reintroduction of $\mathrm{CO}_{2}$ (time $=30$, red). The assemblies reappear in roughly the same locations and with nearly the same geometry. Foci also appear at the meiotic spindle (brighter spots near upper right) (adapted with permission from Gilliland et al. 2009). g Reaction catalyzed by D-amino acid oxidase (EC 1.4.3.3)

gene Tdh3), tetrameric phosphoglycerate mutase (yeast gene Gpm1), enolase I (yeast gene Eno1), synthase subunit of trehalose-6-P synthase/phosphatase complex (yeast gene Tps1), beta subunit of fatty acid synthetase (yeast gene Fas1), alpha subunit of fatty acid synthetase (yeast gene Fas2), and heat shock proteins (yeast genes HSP70, Ssa1, and Ssa2)(Jin et al. 2017). Proteosomal subunits were also found to localize near G-bodies (Jin et al. 2017). G-bodies were found to be required for increased glycolytic demand, and are distinct from stress granules or P-bodies (Jin et al. 2017). G-bodies form specifically from hypoxia, and not 
glucose deprivation (in fact their formation required glucose), nitrogen deprivation, or after being grown on a nonfermentable carbon source (Jin et al. 2017). The intrinsically disordered region (IDR) of PFK1 was found to be important for its colocalization to G-bodies, suggestive of a similarity to factors contributing to other membraneless compartments such as stress granules and P-bodies (Jin et al. 2017). The AMP activated protein kinase Snfl is also required for Gbody formation (Miura et al. 2013; Jin et al. 2017). G-bodies also appear to depend on RNA, as treatment of cells with ribonucleases decreases their formation (Miura et al. 2013), hence reminiscent again of stress granules and P-bodies (Jain et al. 2016; Jin et al. 2017). Relatedly, phosphofructokinase and other enzymes involved in glucose metabolism have been shown to cluster into assemblies or "glucosomes" in human cells (Kohnhorst et al. 2017).

\section{Mps1/Polo kinases}

MPS1 protein kinases are found widely, but not ubiquitously, in eukaryotes. This family of potentially dual-specific (threonine/tyrosine) protein kinases is among several that regulate a number of steps of mitosis. The most widely conserved MPS1 kinase functions involve activities at the kinetochore in both the chromosome attachment and the spindle checkpoint. MPS1 kinases also function at centrosomes. Beyond mitosis, MPS1 kinases have been implicated in development, cytokinesis, and several different signaling pathways. Polo kinases are serine/threonine kinases that participate in 3 metabolic pathways: cell cycle, cell cycle-yeast, and progesteronemediated oocyte maturation.

Gilliland and coworkers report that Mps1 and Polo kinase localize to numerous self-assemblies in the ooplasm of prometaphase Drosophila oocytes (Gilliland et al. 2009). They first appear throughout the oocyte at the end of prophase and are disassembled after egg activation. The self-assemblies form reversibly in response to hypoxia (Fig. 30f). The return to identical positions suggests an unseen filamentous structure in the cell, yet the kinases did not colocalize with tubulin, actin, anillin, septin, or lamin (Pandey et al. 2007). The addition of collagenase prevents filament formation by the kinases, suggesting that the underlying filament is sensitive to this protease. Gilliland and coworkers hypothesize that the self-assemblies formed by Mps 1 and Polo could serve in signaling, that absence of the kinases results in a signal throughout the oocyte (a surrogate kinetochore) (Gilliland et al. 2009).

\section{D-Amino acid oxidase}

DAO catalyzes the oxidation of D-amino acids to their corresponding amino acid (Fig. 30g). The enzyme is most active towards neutral D-amino acids, and not active towards acidic D-amino acids. One of its most important targets in mammals is D-serine in the central nervous system. By targeting this and other D-amino acids in vertebrates, DAO is important in detoxification. The role in microorganisms is slightly different, breaking down D-amino acids to generate energy (Pollegioni et al. 2007). In 1966, Antonini and coworkers used light scattering to demonstrate polymerization of the protein in vitro. Lower $\mathrm{pH}$ and temperature, and higher chloride concentration, decreased this polymerization. Similarly, infinite selfassociation was also shown to best fit sedimentation and light-scattering data of DAO purified from pig kidney (Tojo et al. 1985b; Tojo et al. 1985a).

\section{Additional self-assembly forming enzymes discovered in large-scale screens}

Eighteen additional enzymes, in addition to several discussed above, were uncovered in a screen performed by Narayanaswamy and coworkers as forming punctate foci or rods in yeast under nutrient starvation conditions (stationary growth phase) (Narayanaswamy et al. 2009). Ninety percent of the proteins identified as forming foci in the GFP screen were also found in pelleted fractions of cell lysate (without GFP tags), showing the behavior in the absence of GFP. All proteins listed in Tables 1 and 2 as identified in the Narayanaswamy screen were found to reversibly associate into these foci and dissociate upon addition of nutrients. Those not already discussed above include alanyl-tRNA synthetase, leucyl tRNA synthetase, cystathionine $\beta$-synthase, trifunctional CAD enzyme, adenylosuccinate synthetase, alcohol dehydrogenase, UDP-Glucose-pyrophosphorylase, sterol 3- $\beta$-glucosyltransferase, peptidyl-prolyl cis-trans isomerase, glutamine tRNA synthetase, histidine tRNA synthetase, isoleucine tRNA synthetase, protein kinase of the PAK/Ste20 family, ribosome-associated molecular chaperones, ATPases, threonyl tRNA synthetase, trehalose-6-P synthase/ phosphatase complex, $\gamma$-aminobutyrate (GABA) transaminase, valyl-tRNA synthetase, and serine hydroxymethyltransferase (Narayanaswamy et al. 2009) (Table 2).

A screen by Noree and coworkers, published in 2010, identified another 13 enzymes (in addition to confirmed those seen in prior screens) which form foci upon nutrient starvation (Table 2). These include 5-phospho-ribosyl-1(alpha)-pyrophosphate synthetase, glutamine amidotransferase (GATase II), threonine aldolase, glycogen synthase, glycogen phosphorylase, aminolevulinate dehydratase, multifunctional enzyme containing phosphoribosyl-ATP pyrophosphatase; phosphoribosyl-AMP cyclohydrolase, and histidinol dehydrogenase activities, S-adenosylmethionine synthetase, disaggregase (ATPase), ATPase involved in protein folding and NLS-directed nuclear transport, HSP70 family ATPbinding protein, non-ATPase regulatory subunit of the $26 \mathrm{~S}$ proteasome, homoserine kinase (yeast gene Thr1, also 
Table 1 Summary of activities and filament structures for selected enzymes

\begin{tabular}{|c|c|c|c|c|}
\hline Enzyme & Type of structure & $\begin{array}{l}\text { Regulation of enzyme activity } \\
\text { by assembly }\end{array}$ & Conditions promoting assembly & References \\
\hline \multicolumn{5}{|l|}{ Metabolic enzymes } \\
\hline $\begin{array}{l}\text { Acetyl-CoA carboxylase } 1 \\
\text { and } 2 \text { (ACC1 and ACC2) } \\
\text { (yeast, chicken, mice, human) } \\
\text { Pathway: fatty acid } \\
\text { biosynthesis }\end{array}$ & 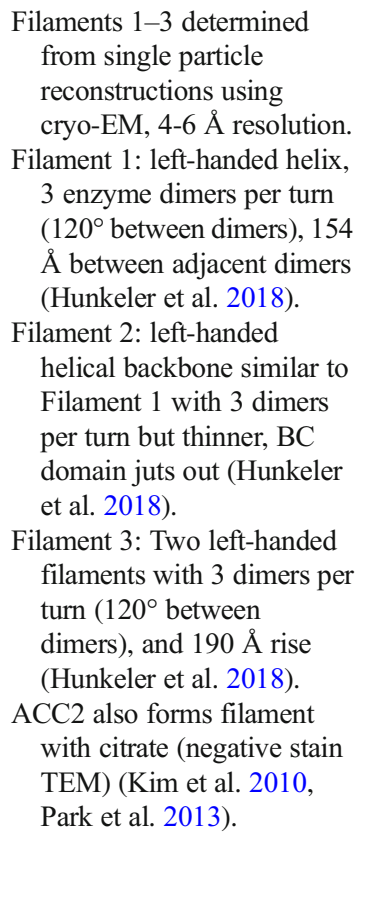 & $\begin{array}{l}\text { Filament 1: citrate but no } \\
\text { ACC phosphorylation, } \\
\text { active enzyme (Hunkeler et } \\
\text { al. 2018). } \\
\text { Filament 2: citrate, no ACC } \\
\text { phosphorylation, } \\
\text { palmitoyl-CoA, inactive } \\
\text { enzyme (Hunkeler et al. } \\
\text { 2018). } \\
\text { Filament 3: BRCT domains of } \\
\text { BRCA1 bound to } \\
\text { phosphorylated ACC1, } \\
\text { inactive enzyme (Hunkeler } \\
\text { et al. 2018). } \\
\text { Filament of ACC1 with } \\
\text { MIG12: } 60 \times \text { activation } \\
\text { (Kim et al. 2010). }\end{array}$ & $\begin{array}{l}\text { Filament 1: } 10 \text { mM citrate and } \\
\text { dephosphorylated ACC1 } \\
\text { (Hunkeler et al. 2018). } \\
\text { Filament 2: 10-fold molar excess } \\
\text { of palmitoyl-CoA to filament } 1 \\
\text { (Hunkeler et al. 2018). } \\
\text { Filament 3: addition of BRCT } \\
\text { domains of BRCA1 to } \\
\text { phosphorylated ACC1 (they } \\
\text { bind 1:1 BRCT domain:ACC1 } \\
\text { dimer) (Hunkeler et al. 2018). } \\
\text { > mM citrate induces filament } \\
\text { formation by both ACC1 and } \\
\text { ACC2 (Kleinschmidt et al. } \\
\text { 1969; Kim et al. 2010; Park et al. } \\
\text { 2013). } \\
\text { MIG12 promotes filamentation of } \\
\text { ACC1 and ACC2 (Kim et al. } \\
\text { 2010). } \\
\text { SPOT14 and MIG12 inhibit } \\
\text { filament formation by ACC1 } \\
\text { and ACC2 (Park et al. 2013). } \\
\text { Forms foci when GFP labeled in } \\
\text { yeast cells under nutrient } \\
\text { starvation conditions (Noree et } \\
\text { al. 2010, Shen et al. 2016). }\end{array}$ & $\begin{array}{l}\text { (Kleinschmidt et } \\
\text { al. 1969) } \\
\text { (Meredith and } \\
\text { Lane 1978) } \\
\text { (Beaty and Lane } \\
\text { 1983) } \\
\text { (Brownsey et al. } \\
\text { 2006) } \\
\text { (Kim et al. 2010) } \\
\text { (Noree et al. } \\
\text { 2010) } \\
\text { (Park et al. 2013) } \\
\text { (Shen et al. 2016) } \\
\text { (Kohnhorst et al. } \\
\text { 2017) } \\
\text { (Jin et al. 2017) } \\
\text { (Hunkeler et al. } \\
\text { 2018) } \\
\text { (Noree et al. } \\
\text { 2019a) }\end{array}$ \\
\hline $\begin{array}{l}\text { ATP-dependent } \\
\text { 6-phosphofructokinase } \\
\text { (yeast genes Pfk1, Pfk2) } \\
\text { (mammals, C. elegans) } \\
\text { Pathway: glycolysis }\end{array}$ & $\begin{array}{l}\text { Single particle reconstruction } \\
\text { from negative stain TEM, } \\
25 \AA \text { resolution. } \\
\text { Filament of stacked tetramers } \\
\text { which can assemble either } \\
\text { of two ways resulting in } \\
\text { either straight or kinked } \\
\text { filament. } \\
\text { Right-handed helix with } \\
\text { stacked PFKL tetramers } \\
\text { related by a rotation of } 221^{\circ} \\
\text { and translation of } 83 \AA \\
\text { between subunits (Webb et } \\
\text { al. } 2017 \text { ). } \\
\text { Average size is } 6 \text { tetramers. } \\
\text { Kink leads to junction of } \sim \\
130^{\circ} \text {. }\end{array}$ & $\begin{array}{l}\text { Effect on enzyme activity not } \\
\text { yet known. }\end{array}$ & $\begin{array}{l}\text { Filaments form in response to } \\
\text { substrate, 6-phosphofuctose (in } \\
\text { vitro) and citrate (in cells) (Webb } \\
\text { et al. 2017). } \\
\text { Forms foci when GFP labeled in } \\
\text { yeast cells under nutrient } \\
\text { starvation or hypoxic conditions } \\
\text { (Noree et al. 2010; Shen et al. } \\
\text { 2016; Jin et al. 2017). } \\
\text { Formed foci at nerve cell synapses } \\
\text { where energy demands are } \\
\text { likely high (Jang et al. 2016). } \\
\text { Found in G-bodies (Jin et al. 2017). }\end{array}$ & $\begin{array}{l}\text { (Kemp 1971) } \\
\text { (Telford et al. } \\
\text { 1975) } \\
\text { (Trujillo and Deal } \\
\text { 1977) } \\
\text { (Reinhart and } \\
\quad \text { Lardy 1980) } \\
\text { (Foe and Trujillo } \\
\text { 1980) } \\
\text { (Schwock et al. } \\
\text { 2004) } \\
\text { (Ranjit et al. } \\
\text { 2014) } \\
\text { (Jang et al. 2016) } \\
\text { (Shen et al. 2016) } \\
\text { (Webb et al. 2017) } \\
\text { (Jin et al. 2017) }\end{array}$ \\
\hline $\begin{array}{l}\text { Glucokinase (yeast gene } \\
\text { Glk1) } \\
\text { (fungi) } \\
\text { Pathway: glycolysis }\end{array}$ & $\begin{array}{l}\text { Filament of S. cerevisiae Glk1 } \\
\text { was resolved to } 3.8 \AA \text { by } \\
\text { cryo-EM and single particle } \\
\text { reconstruction (Stoddard et } \\
\text { al. 2019) and forms a } \\
\text { right-handed, anti-parallel } \\
\text { double helix. }\end{array}$ & $\begin{array}{l}\text { Enzyme is inhibited in the } \\
\text { filament(Stoddard et al. } \\
\text { 2019). }\end{array}$ & $\begin{array}{l}\text { Bundles visible in cells form of } \\
\text { Glk1-GFP fusions in response to } \\
\text { glucose (Stoddard et al. 2019). } \\
\text { Filaments form in response to } \\
\text { substrates (ATP and glucose, } \\
\text { mannose, or glucosamine) or its } \\
\text { products (ADP and } \\
\text { sugar-6-phosphate)(Stoddard et } \\
\text { al. 2019). } \\
\text { Modest filament formation in the } \\
\text { presence of inhibitors } \\
\text { N-acetylglucosamine-6-phosph- } \\
\text { ate and N-acetylglucosamine } \\
\text { (Stoddard et al. 2019). } \\
\text { Filaments do not form in vitro in } \\
\text { the presence of fructose or } \\
\text { galactose (Stoddard et al. 2019). }\end{array}$ & $\begin{array}{l}\text { (Stoddard et al. } \\
\text { 2019) } \\
\text { (Noree et al. } \\
\text { 2019a) }\end{array}$ \\
\hline
\end{tabular}


Table 1 (continued)

CTP synthase (CTPS)

(bacterial, yeast genes Ura7 and Ura8, Drosophila, human)

Pathway: pyrimidine biosynthesis (CTP)
Inosine monophosphate dehydrogenase (IMPDH) (yeast, Drosophila, human) Pathway: nucleotide synthesis (GTP)

\begin{abstract}
Filaments from bacteria and from human are composed of stacked tetrameric CTPS, but they assemble differently. The bacterial CTPS filament was resolved to $4.6 \AA$ by cryo-EM and single particle reconstruction and shows interdigitating tetramers with a rotation of $\sim 49^{\circ}$ between and $\sim 3.7$ per turn (Lynch et al. 2017).

The human CTPS filament was resolved to $6.1 \AA$ by cryo-EM and single particle reconstruction and shows stacked tetramers with less interdigitation than the bacterial form: $\sim 30^{\circ}$ per subunit and $\sim 3$ per turn (Lynch et al. 2017).
\end{abstract}

Filaments were characterized by single particle or helical reconstructions and negative stain TEM (20 Å resolution), and cryo-EM of Y12A IMPDH with GTP $(8.7 \AA$ resolution), and were found to be composed of stacked IMPDH octamers with a slight rotation between them (due to high symmetry, this could be considered either right or lefthanded) (Anthony et al.

2017).

Octamers resemble balls with holes in side, which can be open (assumed to be the active state) or collapsed (assumed to be the inactive) (Anthony et al. 2017).

Filaments of open filaments have a rotation of $30^{\circ}$ between adjacent octamers, with each octamer spanning a distance of 111 $\AA$.

Filaments of collapsed octamers have a rotation of about $35.5^{\circ}$ between adjacent octamers, and one octamer spans a distance of $94 \AA$ (Anthony et al. 2017).

Both open and collapsed octamers appear to be able to coexist in the same filament (Anthony et al. 2017).

Also forms large structures known as "cytoophidia" in cells which may be bundles of filaments (Ji et al. 2006, Carcamo et al. 2011).
Bacterial CTPS is inhibited In bacteria, product (CTP) drives

when in filament (Barry et al. filamentation (Ingerson-Mahar et 2014; Noree et al. 2014).

Human CTPS is activated when in filament (Lynch et al. 2017).

al. 2010).

In human, filaments are favored in presence of substrates UTP and ATP, not products CTP and ADP (Lynch et al. 2017).

Forms foci when GFP labeled in yeast cells under nutrient starvation conditions (Noree et al. 2010, Shen et al. 2016).

Forms cytoophidia in Drosophila (Liu 2010).

Though it is not known, the open form is considered the active form and the collapsed the inactive form (Anthony et al. 2017).

IMPDH was shown to be stimulated 2.7-fold in filaments (Labesse et al. 2013)

ATP induces both types of filament

(Anthony et al. 2017).

GTP, a downstream product (or guanonsine) induces the collapsed type of filament (Anthony et al. 2017).

IMP, a substrate, and $\mathrm{NAD}^{+}$, the cofactor consumed in the reaction, induce the open form
(Werner et al.

2009)

(Noree et al. 2010)

(Liu 2010)

(Ingerson-Mahar et al. 2010)

(Chen et al. 2011)

(Carcamo et al. 2011)

(Barry et al. 2014)

(Aughey et al. 2014)

(Noree et al. 2014)

(Shen et al. 2016)

(Lynch et al. 2017)

(Noree et al. 2019a) (Anthony et al. 2017).

Mycophenolic acid (an inhibitor) induces filaments in cells, GTP disperses filaments in cells, suggesting an inactive state (Nagai et al. 1991; Zimmermann et al. 1998).

MgATP stimulates filamentation in vitro and stimulates enzyme 2.7fold (Labesse et al. 2013). 
Table 1 (continued)

\begin{tabular}{|c|c|c|c|c|}
\hline $\begin{array}{l}\text { Glutamine (Q) synthetase } \\
\text { (yeast gene Gln1) }\end{array}$ & $\begin{array}{l}\text { Forms pentameric rings that } \\
\text { stack into a decamer (two } \\
\text { rings with } 30^{\circ} \text { twist between } \\
\text { them) which further stack into } \\
\text { filaments with } \sim 10^{\circ} \text { twist } \\
\text { between them (Petrovska et al. } \\
\text { 2014). } \\
\text { Forms foci in cells as a result } \\
\text { of nutrient starvation and } \\
\text { heat stress (Noree et al. } \\
\text { 2010). } \\
\text { Correlative light microscopy } \\
\text { and negative stain TEM } \\
\text { shows these to be }\end{array}$ & $\begin{array}{l}\text { Glutamine synthetase is } \\
\text { inactive in the filament } \\
\text { (Petrovska et al. 2014). }\end{array}$ & $\begin{array}{l}\text { Filaments form at low pH } \\
\text { (Petrovska et al. 2014). } \\
\text { Zinc induces decameric rings into } \\
\text { filaments (Miller et al. 1974). } \\
\text { Filamentation in cells is reversible } \\
\text { in the absence and presence of } \\
\text { glucose (Narayanaswamy et al. } \\
\text { 2009). }\end{array}$ & $\begin{array}{l}\text { (Miller et al. } \\
\text { 1974) } \\
\text { (Frey et al. 1975) } \\
\text { (Narayanaswamy } \\
\quad \text { et al. 2009) } \\
\text { (Noree et al. } \\
\quad \text { 2010) } \\
\text { (O'Connell et al. } \\
\text { 2014) } \\
\text { (Petrovska et al. } \\
\quad \text { 2014) } \\
\text { (Shen et al. 2016) } \\
\text { (Noree et al. } \\
\quad 2019 \text { a) }\end{array}$ \\
\hline
\end{tabular}

$\beta$-glucosidase

(plants including oat)

Pathway: host defense
$\mathrm{CO}_{2}$ reductase

(bacteria, Acetobacterium woodii)

Pathway: carbon fixation and energy conservation Nitrilase

(bacteria, fungi, plants)

Pathway: detoxification

\section{CoA-dependent} acetaldehyde and alcohol dehydrogenase (AdhE)

(bacteria, photosynthesizing unicellular organisms) composed of aligned filaments $\sim 1$ micron long (Petrovska et al. 2014).

Negative stain TEM shows trimeric rings that dimerize into hexamers and larger oligomers (Kim et al. 2000; Kim et al. 2005).

Single particle reconstructions suggest a difference in the twist between neighboring trimers forming a "tunnel" housing the active sites. In the multimers, the twist between trimers is $38^{\circ}$. In the hexamers this is $60^{\circ}$.

The smaller twist seems to result in smaller side fenestrations presumably changing access to the active site (Kim et al. 2000, Kim et al. 2005).

Forms fibrils in cells termed stromacenters (Gunning 1965).

Filaments are 10-15 nm, with Filamentous form is 2-fold an apparent helical structure (Schuchmann et al. 2016)

Left-handed helical filament with 4-5 enzyme dimers per turn (Woodward et al. 2018).

Negative stain TEM showing "spiralosomes" of 20-60 in length with left-handed The helical filaments extend

Two-fold lower $\mathrm{K}_{\mathrm{M}}$ and 1.6 lower $\mathrm{k}_{\mathrm{cat}}$ in the filament (Kim et al. 2005)

This effect may be related to steric hindrance of substrates and products entering and leaving the enzyme through openings in the filament vs. the hexamer (tighter binding but slower turnover)(Kim et al. 2005).

more active (Schuchmann et al. 2016)

The filament is more active (Nagasawa et al. 2000).

The helical twist of filaments of nitrilases from different organisms appears to correlate with substrate specificity, hypothesized to be due to substrate binding cleft size (Woodward et al. 2018). and become slimmer upon $\mathrm{Fe}^{2+}$ and $\mathrm{NAD}^{+}$addition. Authors speculate that this is the active form, because it is
Activated upon fungal infection. (Gunning 1965)

Disassembled by $\mathrm{CaCl}_{2}$ (Kim et al. (Nisius and 2005).

Ruppel 1987)

(Nisius 1988)

(Kim et al. 2000)

(Kim et al. 2005)

Filaments are induced by $5-20 \mathrm{mM}$ (Schuchmann et $\mathrm{MgSO}_{4}, 20 \mathrm{mM} \mathrm{MnCl}$ or $\mathrm{CaCl}_{2}$ al. 2016) (Schuchmann et al. 2016).

$10 \mathrm{mM}$ benzonitrile, but not aliphatic nitriles, induced filamentation and increased activity of the enzyme (Nagasawa et al. 2000).

$10 \%$ saturated ammonium sulfate and $50 \%(\mathrm{v} / \mathrm{v})$ glycerol,

increased temperatures, or enzyme concentrations induces the activated filament (Nagasawa et al. 2000).

Wounding of plants (cutting or with herbicides) induces foci formation in plant cells (Cutler and Somerville 2005).

Filaments found in cells, however (Kessler et al. ligands $\left(\mathrm{Fe}^{3+}, \mathrm{NAD}^{+}\right)$can induce 1991) structural changes in helical filaments (Kessler et al. 1992).
(Harper 1977b; Harper 1977a)

(Cutler and Somerville 2005)

(Thuku et al. 2007)

(Thuku et al. 2009)

(Chan et al. 2011)

(Woodward et al. 2018)

(Kessler et al. 1992) 
Table 1 (continued)

\begin{tabular}{|c|c|c|c|c|}
\hline Pathway: fermentation & $\begin{array}{l}\text { geometry (Kessler et al. 1992; } \\
\text { Laurenceau et al. 2015). } \\
\text { Model building with } 7 \text { copies } \\
\text { per turn with right-handed } \\
\text { helical geometry (Extance } \\
\text { et al. 2013). }\end{array}$ & $\begin{array}{l}\text { more open, allowing } \\
\text { substrates and products access } \\
\text { to the enzymes, whereas the } \\
\text { wider, shorter, more compact } \\
\text { forms may be the inactive } \\
\text { form of the enzyme (Kessler } \\
\text { et al. 1992). }\end{array}$ & & $\begin{array}{l}\text { (Extance et al. } \\
\text { 2013) } \\
\text { (Laurenceau et al. } \\
\text { 2015) }\end{array}$ \\
\hline $\begin{array}{l}\text { Glutamate }(\mathrm{E}) \\
\text { dehydrogenase } \\
\text { (yeast gene Gdh2, mammals) } \\
\text { Pathway: nitrogen and } \\
\text { glutamate metabolism, } \\
\text { energy homeostasis, amino } \\
\text { acid synthesis, and in } \\
\text { plants tolerance to } \\
\text { herbicide, water deficit, } \\
\text { pathogen infections }\end{array}$ & $\begin{array}{l}\text { Helical tubes in vitro } \\
\text { visualized by negative stain } \\
\text { TEM (9-9.3 subunits per turn) } \\
\text { (Josephs and Borisy 1972). }\end{array}$ & $\begin{array}{l}\text { Though the effect on enzyme } \\
\text { activity of filamentation is not } \\
\text { known, conditions activating } \\
\text { the enzyme also induce } \\
\text { filamentation, and conditions } \\
\text { deactivating the enzyme } \\
\text { dissociate the filament, } \\
\text { suggesting that filamentation } \\
\text { increases enzyme activity } \\
\text { (Frieden 1959b, Gylfe 1976, } \\
\text { Fahien et al. 1989). }\end{array}$ & $\begin{array}{l}\text { Stationary phase (i.e., nutrient } \\
\text { starvation) induces cytoophidia in } \\
\text { yeast (Shen et al. 2016). } \\
\text { GTP and NADH induce } \\
\text { depolymerization (and are } \\
\text { inhibitors) in vitro (Huang and } \\
\text { Frieden 1972; Zeiri and Reisler } \\
\text { 1978). } \\
\text { ADP favors polymerization (and is } \\
\text { an activator) in vitro (Huang and } \\
\text { Frieden 1972, Zeiri and Reisler } \\
\text { 1978). }\end{array}$ & $\begin{array}{l}\text { (Olson and } \\
\text { Anfinsen 1952) } \\
\text { (Eisenberg and } \\
\text { Reisler 1971) } \\
\text { (Huang and } \\
\text { Frieden 1972) } \\
\text { (Josephs and } \\
\text { Borisy 1972) } \\
\text { (Zeiri and Reisler } \\
\text { 1978) } \\
\text { (Shen et al. 2016) } \\
\text { (Noree et al. } \\
\text { 2019a) }\end{array}$ \\
\hline $\begin{array}{l}\text { Glutaminase } \\
\text { (mammals) } \\
\text { Pathway: nitrogen } \\
\text { metabolism }\end{array}$ & $\begin{array}{l}\text { Negative stain TEM ( } 35 \AA \\
\text { resolution) with single } \\
\text { molecule reconstruction and } \\
\text { model fitting showing right- } \\
\text { handed double helix with } 53 \pm\end{array}$ & $\begin{array}{l}\text { Filaments have increased } \\
\text { catalytic activity (Ferreira et } \\
\text { al. 2013). }\end{array}$ & $\begin{array}{l}\text { Formed in vitro with purified } \\
\text { protein, phosphate and phosphate- } \\
\text { borate added to protein in Tris-HCl } \\
\text { induces polymers (Olsen et al. } \\
\text { 1970). }\end{array}$ & $\begin{array}{l}\text { (Olsen et al. 1970) } \\
\text { (Ferreira et al. } \\
\text { 2013) } \\
\text { (Petrovska et al. } \\
\text { 2014) }\end{array}$ \\
\hline
\end{tabular}

$2 \mathrm{~nm}$ rise per turn, strand inclination of $25^{\circ}$, and an average width for a single strand of $6.6 \pm 0.7 \mathrm{~nm}$ (Ferreira et al. 2013).

$\beta$-lactamase-like protein

(mammals)

Pathway: unknown

2-Cys peroxiredoxins

(archea, bacteria, eukaryotes)

Pathway: $\mathrm{H}_{2} \mathrm{O}_{2}$ metabolisom

and detoxification, protein

folding stress (LACTB)
Appears as a braided filament in negative stain TEM (Polianskyte et al. 2009).

Filament has hollow core that binds unfolded proteins.

Formed from stacks of decameric rings viewed by negative stain TEM (Gourlay et al. 2003).
Enzyme activity has not been investigated, and therefore it is not known how filamentation affects enzyme activity.

Filaments are inactive in peroxidase activity, but active in chaperone activity (binding unfolded proteins,

sequestering them to prevent aggregation).
Filaments are found in the intracristal part of the intermembrane space of mitochondria, spanning the cristae.

Redox stress, high hydrogen peroxide levels, heat shock, low $\mathrm{pH}$, and site-specific phosphorylation induce filamentation.
Polianskyte et al. 2009)

(Wood et al.

2002)

(Gourlay et al. 2003)

(Angelucci et al. 2013)

(Phalen et al. 2006)

(Saccoccia et al. 2012)

(Angelucci et al. 2013)

(Puerto-Galan et al. 2013)

(Noichri et al. 2015)

(Teixeira et al. 2019)

(Narayanaswamy et al. 2009)

$\begin{array}{cl}\text { Ribonucleotide reductase } & \text { Cryo-EM, } 2 \text { types of } \\ \text { (archea, bacteria, eukaryotes; } & \text { filaments, } 4.7-4.8 \AA\end{array}$ yeast genes Rnr2 and Rnr4)

Pathway: nucleotide metabolism

resolution, one a double helix al. 2019).

(right-handed), the other having a filament of $\mathrm{NrdE}$ entwinned with a filament of $\mathrm{NrdF}$ (also righthanded)(Thomas et al. 2019).

Nucleic acid enzymes SgrAI

(bacterial: Streptomyces griseus)
Helical reconstruction and cryo-EM (3.5 Å resolution) shows left handed helix with $\sim$
Enzyme is inhibited in both High dATP $(100 \mu \mathrm{M})$ induces types of filaments (Thomas et filaments (Thomas et al. 2019).

Filaments differ with respect to the presence or absence of $\mathrm{NrdF}$ protein (Thomas et al. 2019).

Punctate foci seen in yeast with nutrient starvation (Narayanaswamy et al. 2009).

The enzyme is $200-1000 \times \quad$ Binding to double-stranded DNA activated in the filament (Park containing the primary site et al. 2010; Shah et al. 2015). sequence CRCCGGYG, and at
(Ando et al. 2016)

(Thomas et al. 2019)

(Noree et al. 2019a)

(Park et al. 2010) (Shah et al. 2015) 
Table 1 (continued)

\begin{tabular}{ll}
\hline Pathway: host defense & 4 DNA bound enzyme dimers \\
& per turn $\left(\sim 90^{\circ}\right)$ (Polley et al. \\
& $2019)$.
\end{tabular}
2019).

\section{IRE1}

(eukaryotes)

Pathway: unfolded protein response, response to stress
X-ray crystal structure

showed right-handed helical

filament with 7 dimeric forms

of the IRE1 chain per turn ( $50^{\circ}$ ) (Korennykh et al. 2009). Also forms foci in live cells

(Aragon et al. 2009,

Korennykh et al. 2009, Li

et al. 2010, Ishiwata-

Kimata et al. 2013).

\section{RecA}

(E. coli)

Pathway: homologous recombination

DnaA

(E. coli)

Pathway: DNA replication initiation
Right-handed helical

filaments on DNA have been characterized by electron microscopy and x-ray crystallography $(2.8-4.3 \AA$ resolution) with $\sim 60^{\circ}$ per turn, $94 \AA$ pitch, $\sim 6$ RecA proteins per turn (Chen, Yang, et al. 2008). This filament has polarity (the two ends are not equivalent).

Right handed helical filaments on DNA have been

characterized by $\mathrm{x}$-ray crystallography with $45^{\circ}$ per turn, $178 \AA$ pitch, 8 copies per turn (Erzberger et al. 2006, Duderstadt et al. 2011). This filament has polarity (the two ends are not equivalent).

Kinases and innate immunity signaling filaments Casein kinase CK2 (human)

Pathway: cell cycle control, cellular differentiation, and proliferation, circadian rhythm, apoptosis, and gene expression Adenylate kinase (bacteria, plants) Pathway: nucleotide balance

RIP1/RIP3 kinase (mammals) Protomer is heterotetramer $\left(\alpha_{2} \beta_{2}\right)$. Oligomers are deduced from native MS, IMMS, and H/D exchange (Seetoh et al. 2016). X-ray crystal structure shows linear form (Schnitzler et al. 2014). X-ray crystal structure shows linear filament with no helicity form from stacked hexamers (Wild et al. 1997).

Negative stain TEM. RIP homotypic interaction motif
Filamentation activates splicing of TF mRNA 100,000-fold and possibly degradation of other mRNAs (Korennykh et al. 2009). Upon dimerization via binding unfolded proteins, the kinase domains transautophosphorylate lead to phosphorylated sites that likely stabilize the filamentous form (Korennykh et al. 2009).

Binding to ADP also favors filamentation (Korennykh et al. 2009).

Binding to ssDNA and ATP stimulates its filamentation and binding to dsDNA, which is stretched, causing duplex melting, allowing invasion by the single stranded DNA, good base pairing results in ATP hydrolysis and dissociation from the DNA sites in the origin of replication induces

filamentation, which stretches and/or supercoils the DNA inducing strand melting (that allows the replicative helices to be loaded)

Filament is likely the inactive form of the enzyme (Valero et al. 1995).

Forms linear polymer in crystal packing.

Not clear this occurs in vivo. Speculated to inhibit the activity based on steric/ conformation arguments.

Kinase activity activated by assembly (Cho et al. 2009, He least 7 base pairs on either side, (Lyumkis et al.

induce filamentation.

2013)

Double-stranded DNA containing secondary site. sequences CCCCGGYG or XRCCGGYG will be bound by the enzyme, and will not induce

filamentation but will join

filaments formed by enzyme bound to primary site DNA.

Binding to unfolded proteins induces kinase activity that induces oligomerization, which then binds and splices particular mRNAs (Credle et al. 2005; Aragon et al. 2009; Korennykh et al. 2009;

Gardner and Walter 2011; Walter and Ron 2011; Karagoz et al. 2017).

In the filamentous form, the enzyme may cleave many mRNAs nonspecifically (i.e., RIDD activity), while in the dimeric form it cleaves only the particular mRNAs for transcription factors that induce the unfolded protein response pathway.

Binding to ssDNA and ATP stimulates its filamentation which (Park et al. 2018a)

(Park et al. 2018b)

(Barahona et al. 2019)

(Polley et al. 2019)

(Korennykh et al. 2009)

(Li et al. 2010)

(Ghosh et al. 2014) can then bind to dsDNA and perform its activity.

Binding to ATP and specific sites stimulates filamentation which

(Erzberger et al. 2006) gives it its activity

(Chen, Yang, et al. 2008)

(Duderstadt et al. 2011)

High ionic strength (e.g., > $0.5 \mathrm{M}$ $\mathrm{NaCl}$ ) disrupts filaments (Valero et 1995) al. 1995).

Ring form is favored in saturating (Seetoh et al. concentration of substrates (Valero et al. 1995).

Thought to occur at night, when enzyme activity is not needed, and to release water, though not understood how this is regulated (Wild et al. 1997).

Signaling induces association.

(Cho et al. 2009) (He et al. 2009a)

.

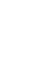


Table 1 (continued)

\begin{tabular}{|c|c|c|c|c|}
\hline $\begin{array}{l}\text { Pathway: host defense, innate } \\
\text { immunity }\end{array}$ & $\begin{array}{l}\text { (RHIM) is a 5-6 residue } \\
\text { segment that forms cross-beta } \\
\text { amyloid structure upon self- } \\
\text { association or association of } \\
\text { RIP1 with RIP3 (Li et al. } \\
\text { 2012a, Mompean et al. 2018). }\end{array}$ & $\begin{array}{l}\text { et al. 2009a, Zhang et al. } \\
\text { 2009). }\end{array}$ & $\begin{array}{l}\text { The purpose of amyloid/fibril } \\
\text { formation may be for feed- } \\
\text { forward gain of function in } \\
\text { which kinase activation and } \\
\text { RIP1/RIP3 necrosome } \\
\text { formation are mutually } \\
\text { reinforcing (Li et al. 2012a). } \\
\text { Upon amyloid formation, } \\
\text { transphosphorylation occurs } \\
\text { which activates RIP1/RIP3 (Li } \\
\text { et al. 2012a). }\end{array}$ & $\begin{array}{l}\text { (Zhang et al. } \\
\text { 2009) } \\
\text { (Li et al. 2012a) } \\
\text { (Mompean et al. } \\
\text { 2018) }\end{array}$ \\
\hline $\begin{array}{l}\text { Death domain containing } \\
\text { (vertebrates) } \\
\text { Pathway: host defense, cell } \\
\text { death, innate immunity }\end{array}$ & $\begin{array}{l}\text { Structures solved via helical } \\
\text { reconstruction cryo-EM, } \\
\text { single particle reconstruction } \\
\text { cryo-EM and x-ray } \\
\text { crystallography. Filaments } \\
\text { assemble via death domain } \\
\text { (CARD, PYD, DED). Some } \\
\text { right-handed, others left- } \\
\text { handed. Can appear as a triple } \\
\text { helix. }\end{array}$ & $\begin{array}{l}\text { Filaments are the activated } \\
\text { form of these signaling } \\
\text { proteins. }\end{array}$ & $\begin{array}{l}\text { Proteins in this category have } \\
\text { CARD or Death Domains that } \\
\text { form filaments. Part of innate } \\
\text { immunity in humans. Form in } \\
\text { response to signal, often a ligand } \\
\text { that induces dimerization of a } \\
\text { death-domain containing protein, } \\
\text { leading to seeding filament that is } \\
\text { formed by another death-domain } \\
\text { containing protein, which } \\
\text { amplifies the signal and creates a } \\
\text { scaffold for activation of } \\
\text { downstream proteins, such as } \\
\text { caspase-1 or kinases, leading to } \\
\text { outcome such as increased } \\
\text { transcription of inflammatory } \\
\text { genes or induction of apoptosis or } \\
\text { necrosis. }\end{array}$ & $\begin{array}{l}\text { (Lin et al. 2010) } \\
\text { (Wu et al. 2014) } \\
\text { (Lu et al. 2014a) } \\
\text { (Diebolder et al. } \\
\quad 2015 \text { ) } \\
\text { (Lu et al. 2016) } \\
\text { (Gong et al. 2018) }\end{array}$ \\
\hline $\begin{array}{l}\text { TORC1 } \\
\text { (yeast) } \\
\text { Pathway: nutrient/energy/ } \\
\text { redox sensor and controls } \\
\text { protein synthesis }\end{array}$ & $\begin{array}{l}\text { Negative stain EM single } \\
\text { particle reconstruction }(27 \AA \\
\text { resolution). Hollow tube or } \\
\text { cylinder called TOROID, } \\
\text { right-handed with } \sim 9 \text { copies } \\
\text { per turn (Prouteau et al. } 2017 \text { ). }\end{array}$ & $\begin{array}{l}\text { Kinase activity is inactive in } \\
\text { TOROID filament (Prouteau } \\
\text { et al. 2017). }\end{array}$ & $\begin{array}{l}\text { Glucose starvation (Prouteau et al. } \\
\text { 2017). } \\
\text { Dependent on Rag GTPases for } \\
\text { assembly (Prouteau et al. 2017). }\end{array}$ & $\begin{array}{l}\text { (Prouteau et al. } \\
\text { 2017) }\end{array}$ \\
\hline
\end{tabular}

Death domain containing (vertebrates)

Pathway: host defense, cell particle reconstruction crystallography. Filaments handed. Can appear as a triple helix.
Filaments are the activated form of these signaling immunity in humans. Form in response to signal, often a ligand eading to seeding filament amplifies the signal and creates a caspase-1 or kinases, leading to outcome such as increased

2017)

Dependent on Rag GTPases for right-handed with $\sim 9$ copies (Prouteau et al. 2017).
(Thayer et al. 2014)), and glycogen debranching enzyme (Noree et al. 2010). In addition, Werner and coworkers identified UDP-N-acetylmuramate-alanine ligase as a cellular selfassembly-former (Werner et al. 2009). In 2014, a protein of unknown function with glutathione-S-transferase domain (Drosophila gene Fax, for Failed axon connections (CPTI002774)) was found to form foci in the developing oocyte (Lowe et al. 2014) (Table 2). In 2015, Suresh and coworkers discovered that the fatty acid synthase complex (yeast genes Fas1 and Fas2) form distinct foci under starvation conditions, and that these contain active enzymes and are not substrates of quality control proteins such as Hsp104 and did not colocalize with self-assemblies of Ura7, Pre6, or Ade4 (Suresh et al. 2015). They were also reversible, dispersing in the presence of added nutrients. In 2016, Shen and coworkers confirmed foci formation by many enzymes previously discovered, and also identified thioredoxin peroxidase as forming self-assemblies (Shen et al. 2016).

In 2019, Noree and coworkers reported a quantitative screen in yeast examining foci formation in three growth phases: log phase, after post-diauxic shift, and saturation. Many enzymes uncovered in prior screens were confirmed here, along with several newly identified enzymes including the proteins of yeast genes Aro1, Cha1, Gdh1, Pro3, Prs3, Prs5, Ilv1, Ilv2, Ald6, Adh3, Ald4, Ald5, Gre3, Pdc1, Tal1, Sec53, Tpi1, Faa4, Acs1, Bna5, Dph2, Hem13, Trr1, Coq5, Fum1, Idh2, Kgd1, Lpd1, Mdh1, Mdh2, Pda1, Rip1, Sdh4) (Noree et al. 2019a) (Table 2). Ten of these were found in mitochondria (yeast genes Adh3, Ald4, Ald5, Cha1, Fum1, Ilv1, Ilv2, Kgd1, Mdh1, Pda1) and appeared to concentrate in regions of high respiratory activity. Most were found under conditions of nutrient depletion (in saturated cultures), but some were more prominent in log phase growth (Ilv1, Pda1), or after the post-diauxic shift and before saturation (Pro3, Adh3, Gre3, Hem13, Pda1). The gene product of the gene Ade4 (amidophosphoribosyltransferase, involved in de novo purine biosynthesis) assembled when grown to log phase and shifted to Ade- media, suggesting an increase in activity in the self-assembly. The opposite behavior was seen with the PRPP enzyme, also involved in purine biosynthesis (yeast 
Table 2 Enzymes that form assemblies (in cells or in vitro), but structure and/or regulatory properties not yet determined

\begin{tabular}{|c|c|c|c|c|}
\hline Enzyme/organism & $\begin{array}{l}\text { Type of assembly observed or } \\
\text { image }\end{array}$ & $\begin{array}{l}\text { Conditions triggering assembly in } \\
\text { cells }\end{array}$ & $\begin{array}{l}\text { Is the } \\
\text { assembly } \\
\text { reversible? }\end{array}$ & References \\
\hline \multicolumn{5}{|c|}{ Amino acid metabolism, protein synthesis, protein folding, protein degradation } \\
\hline $\begin{array}{l}\text { Glutamate }(E) \text { synthase } \\
\text { (yeast gene Glt1) } \\
\text { Pathway: ammonia assimilation cycle/glutamate } \\
\text { biosynthetic process }\end{array}$ & $\begin{array}{l}\text { Cytoophidia, rods, and } \\
\text { punctate foci }\end{array}$ & $\begin{array}{l}\text { Similar in stationary phase, diauxic } \\
\text { phase, and log phase (Noree et al. } \\
\text { 2010), more in stationary phase } \\
\text { (Shen et al. 2016) }\end{array}$ & $\begin{array}{l}\text { Shown to be } \\
\text { motile, } \\
\text { undergo } \\
\text { sub-- } \\
\text { diffusion }\end{array}$ & $\begin{array}{l}\text { (Noree et al. } \\
\quad 2010 \text { ) } \\
\text { (Shen et al. 2016) } \\
\text { (Noree et al. } \\
\quad \text { 2019a) }\end{array}$ \\
\hline $\begin{array}{l}\text { Asparagine synthetase } \\
\text { (yeast gene Asn1, Asn2) } \\
\text { Pathway: amino acid metabolism, nitrogen } \\
\text { metabolism }\end{array}$ & Cytoophidia and rods & $\begin{array}{l}\text { Increasing on going from log, to } \\
\text { diauxic and stationary phases, } \\
\text { glucose deprivation }\end{array}$ & Yes & $\begin{array}{l}\text { (Narayanaswamy } \\
\text { et al. 2009) } \\
\text { (Shen et al. 2016) } \\
\text { (Zhang et al. } \\
\text { 2018) } \\
\text { (Noree et al. } \\
\text { 2019a) }\end{array}$ \\
\hline $\begin{array}{l}\text { Alanyl-tRNA synthetase } \\
\text { (yeast gene Ala1) } \\
\text { Pathway: protein synthesis, ala-tRNA } \\
\text { aminoacylation }\end{array}$ & \multicolumn{4}{|c|}{$\begin{array}{l}\text { Pathway: protein synthesis, ala-tRNA } \\
\text { aminoacylation }\end{array}$} \\
\hline $\begin{array}{l}\text { Leucyl tRNA synthetase } \\
\text { (yeast gene Cdc60) }\end{array}$ & Punctate foci & Stationary phase & Yes & $\begin{array}{l}\text { (Narayanaswamy } \\
\text { et al. 2009) }\end{array}$ \\
\hline \multicolumn{5}{|l|}{$\begin{array}{l}\text { Pathway: protein synthesis, Leu-tRNA } \\
\text { aminoacylation }\end{array}$} \\
\hline $\begin{array}{l}\text { Cystathionine } \beta \text {-synthase } \\
\text { (yeast gene Cys } 4 \text { ) }\end{array}$ & Punctate foci & Stationary phase & Yes & $\begin{array}{l}\text { (Narayanaswamy } \\
\text { et al. 2009) }\end{array}$ \\
\hline $\begin{array}{l}\text { Pathway: amino acid metabolism, cysteine } \\
\text { biosynthesis }\end{array}$ & & & & $\begin{array}{l}\text { (Noree et al. } \\
\text { 2019a) }\end{array}$ \\
\hline $\begin{array}{l}\text { eIF2B, eIF2 } \\
\text { (yeast genes Gcd1 (eIF2b- } \gamma, \text { Gcd2 (eIF2B- } \delta), \text { Gcd7 } \\
(\text { IF2B- } \beta \text { ), Gcd6 (eIF2B- } \varepsilon) \text {, and Sui2 (eIF2- } \alpha) \text {, } \\
\text { Gcn3 (eIF2B- } \alpha) \text { ) }\end{array}$ & $\begin{array}{l}\text { Cytoophidia, rods, and } \\
\text { punctate foci }\end{array}$ & $\begin{array}{l}\text { Log and stationary phases, } \\
\text { cycloheximide, acidification }\end{array}$ & Yes & $\begin{array}{l}\text { (Noree et al. } \\
\quad 2014 \text { ) } \\
\text { (Shen et al. 2016) } \\
\text { (Nuske et al. } \\
\quad 2018 \text { ) }\end{array}$ \\
\hline $\begin{array}{l}\text { Peptidyl-prolyl cis-trans isomerase (cyclophilin) } \\
\text { (yeast gene Cpr6) }\end{array}$ & Punctate foci & Stationary phase & Yes & $\begin{array}{l}\text { (Narayanaswamy } \\
\text { et al. 2009) }\end{array}$ \\
\hline \multicolumn{5}{|l|}{ Pathway: Protein folding } \\
\hline $\begin{array}{l}\text { Glutamine tRNA synthetase } \\
\text { (yeast gene Gln4) }\end{array}$ & Punctate foci & Stationary phase & Yes & $\begin{array}{l}\text { (Narayanaswamy } \\
\text { et al. 2009) }\end{array}$ \\
\hline \multicolumn{5}{|l|}{$\begin{array}{l}\text { Pathway: protein synthesis, aminoacylating } \\
\text { appropriate tRNA with glutamine }\end{array}$} \\
\hline $\begin{array}{l}\text { Histidine tRNA synthetase } \\
\text { (yeast gene Hts1) }\end{array}$ & Punctate foci & Stationary phase & Yes & $\begin{array}{l}\text { (Narayanaswamy } \\
\text { et al. 2009) }\end{array}$ \\
\hline \multicolumn{5}{|l|}{$\begin{array}{l}\text { Pathway: protein synthesis, aminoacylating } \\
\text { appropriate tRNA with histidine }\end{array}$} \\
\hline $\begin{array}{l}\text { Isoleucine tRNA synthetase } \\
\text { (yeast gene Ils1) }\end{array}$ & Punctate foci & Stationary phase & Yes & $\begin{array}{l}\text { (Narayanaswamy } \\
\text { et al. 2009) }\end{array}$ \\
\hline \multicolumn{5}{|l|}{$\begin{array}{l}\text { Pathway: protein synthesis, aminoacylating } \\
\text { appropriate tRNA with isoleucine }\end{array}$} \\
\hline $\begin{array}{l}\text { Ribosome-associated molecular chaperones, } \\
\text { ATPases } \\
\text { (yeast genes Ssb1, Ssb2) }\end{array}$ & Punctate foci & Stationary phase & Yes & $\begin{array}{l}\text { (Narayanaswamy } \\
\text { et al. 2009) }\end{array}$ \\
\hline \multicolumn{5}{|l|}{ Pathway: protein biosynthesis } \\
\hline $\begin{array}{l}\text { Threonyl tRNA synthetase } \\
\text { (yeast gene Ths1) }\end{array}$ & Punctate foci & Stationary phase & Yes & $\begin{array}{l}\text { (Narayanaswamy } \\
\text { et al. 2009) }\end{array}$ \\
\hline \multicolumn{5}{|l|}{$\begin{array}{l}\text { Pathway: protein synthesis, aminoacylating } \\
\text { appropriate tRNA with threonine }\end{array}$} \\
\hline $\begin{array}{l}\text { Valyl-tRNA synthetase } \\
\text { (yeast gene Vas1) }\end{array}$ & Punctate foci & Stationary phase & Yes & $\begin{array}{l}\text { (Narayanaswamy } \\
\text { et al. 2009) }\end{array}$ \\
\hline \multicolumn{5}{|l|}{$\begin{array}{l}\text { Pathway: protein synthesis, aminoacylating } \\
\text { appropriate tRNA with threonine }\end{array}$} \\
\hline $\begin{array}{l}\text { Threonine aldolase } \\
\text { (yeast gene Gly1) } \\
\text { Pathway: glycine biosynthesis }\end{array}$ & Punctate foci & Stationary phase & $\begin{array}{l}\text { No } \\
\text { informa- } \\
\text { tion } \\
\text { provided }\end{array}$ & $\begin{array}{l}\text { (Noree et al. } \\
\text { 2010) } \\
\text { (Noree et al. } \\
\text { 2019a) }\end{array}$ \\
\hline $\begin{array}{l}\text { Multifunctional enzyme containing } \\
\text { phosphoribosyl-ATP pyrophosphatase; }\end{array}$ & Punctate foci & Stationary phase & No & $\begin{array}{l}\text { (Noree et al. } \\
2010 \text { ) }\end{array}$ \\
\hline
\end{tabular}


Table 2 (continued)

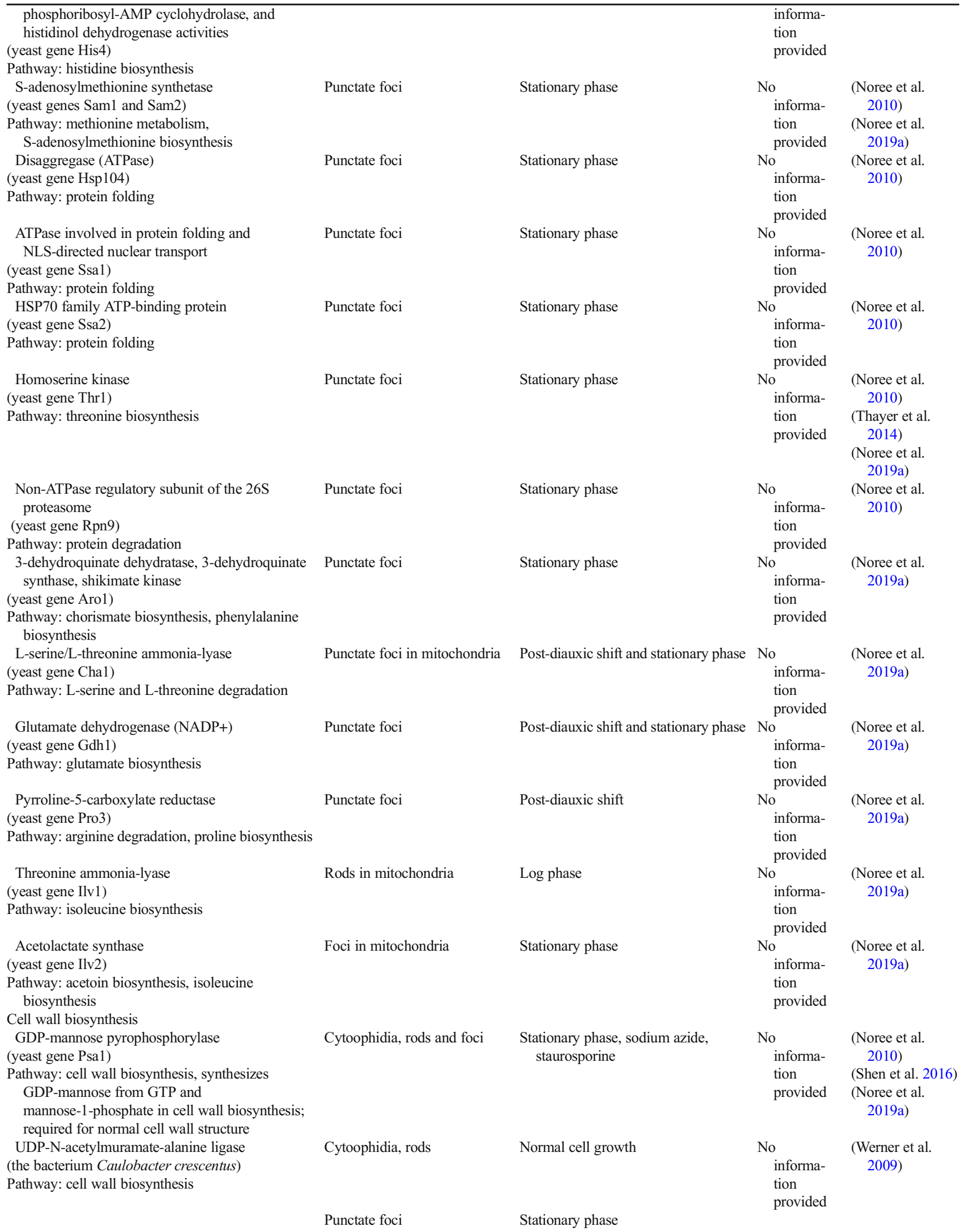


Table 2 (continued)

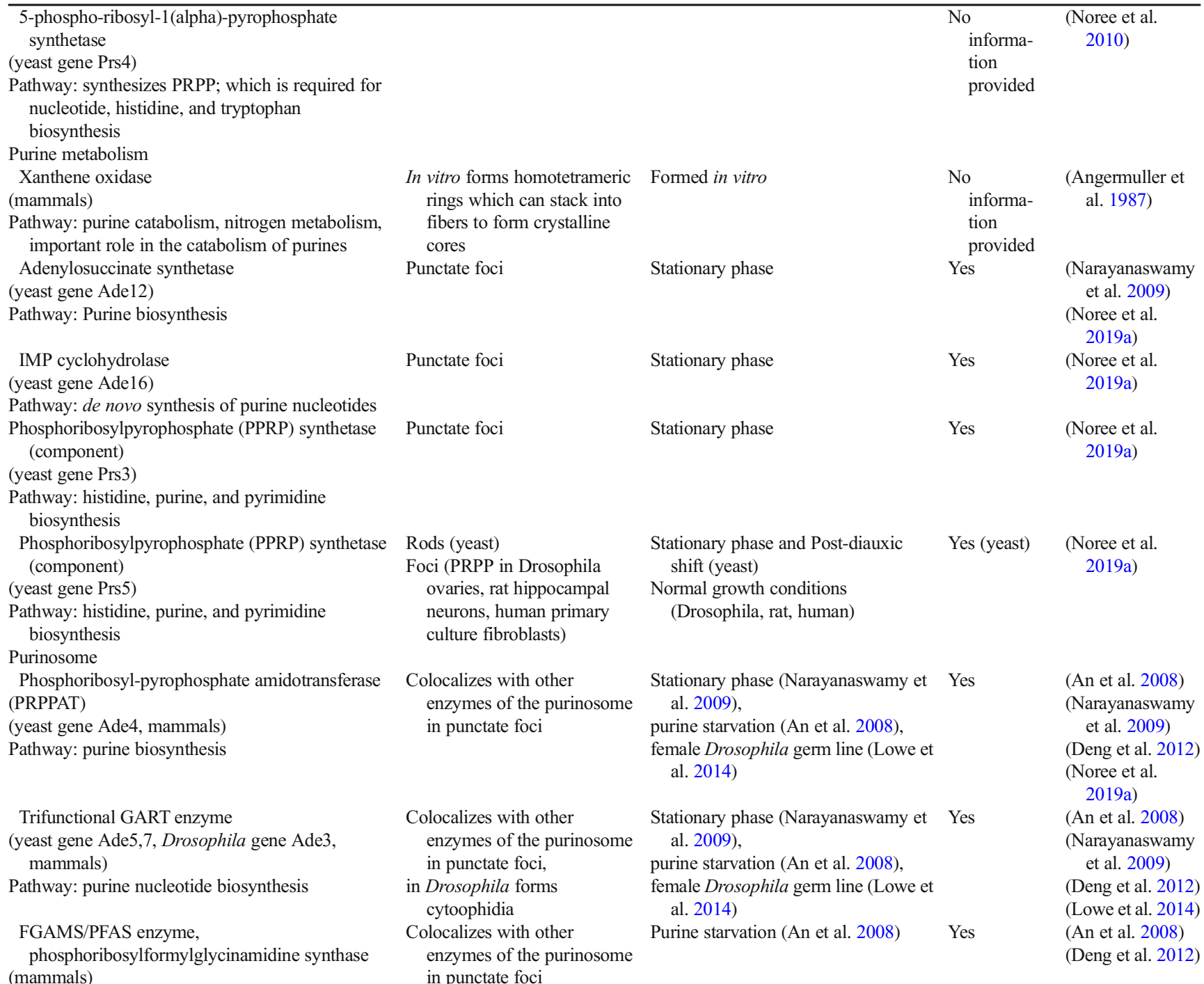

Pathway: purine biosynthesis, reaction step 4 of purinosome

Bifunctional PAICS enzyme

(yeast gene Ade2, Drosophila gene Ade5, mammals)

Pathway: purine nucleotide biosynthesis

ADSL enzyme

(mammals)

Pathway: purine biosynthesis

Bifunctional ATIC enzyme

(yeast gene Ade17, mammals)

Pathway: purine biosynthesis

Pyrimidine biosynthesis

Trifunctional CAD enzyme

(carbamoyl-phosphate synthetase 2, aspartate

transcarbamylase, and dihydroorotase)

(yeast gene Ura2)

Pathway: Pyrimidine synthesis

Punctate foci
Stationary phase (Narayanaswamy et Yes al. 2009),

enzymes of the purinosome in punctate foci,

in Drosophila forms cytoophidia

Colocalizes with other enzymes of the purinosome in punctate foci

Colocalizes with other enzymes of the purinosome in punctate foci purine starvation (An et al. 2008),

female Drosophila germ line (Lowe et al. 2014)

Purine starvation (An et al. 2008) Yes

Stationary phase(Narayanaswamy et Yes al. 2009), purine starvation(An et al. 2008)

(An et al. 2008)

(Narayanaswamy et al. 2009)

(Deng et al. 2012)

(Lowe et al. 2014)

(An et al. 2008)

(Deng et al. 2012)

(An et al. 2008)

(Narayanaswamy et al. 2009)

(Deng et al. 2012)

(Noree et al. 2019a)

(Narayanaswamy et al. 2009) 
Table 2 (continued)

\footnotetext{
Carbohydrate metabolism

Glycogen debranching enzyme

(yeast gene Gdb1)

Pathway: carbohydrate metabolism, contains glucanotranferase and alpha-1,6amyloglucosidase activities; required for glycogen degradation

Pyruvate kinase

(yeast gene Cdc19, mammals)

Pathway: carbohydrate metabolism
}

Enolase I

(yeast gene Eno1)

Pathway: carbohydrate metabolism

Enolase II

(yeast-gene Eno2)

Pathway: carbohydrate metabolism

Fructose bisphosphate aldolase

(yeast gene Fba1)

Pathway: carbohydrate metabolism

Glyceraldehyde-3-phosphate dehydrogenase

(GAPDH)

(yeast gene Tdh3)

Pathway: carbohydrate metabolism

Phosphoglucose isomerase

(yeast gene Pgi1)

Pathway: carbohydrate metabolism

Tetrameric phosphoglycerate mutase

(yeast gene Gpm1)

Pathway: carbohydrate metabolism

Trehalose-6-P synthase/phosphatase complex (synthesis subunit)

(yeast gene Tps1)

Pathway: carbohydrate metabolism, response to desication

Trehalose-6-P synthase/phosphatase complex (yeast gene Tps2)

Pathway: response to stress, synthesis of the storage carbohydrate trehalose

Alcohol dehydrogenase

(yeast gene Adh2)

Pathway: carbohydrate metabolism

\footnotetext{
UDP-Glucose-pyrophosphorylase (yeast gene Ugp1)

Pathway: carbohydrate metabolism

Glycogen synthase

(yeast gene Gsy1)

Pathway: glycogen biosynthesis

Glycogen phosphorylase

(yeast gene Gph1)

Pathway: glycogen catabolism

Aldehyde dehydrogenase (NADP+)

(yeast gene Ald6)

Pathway: glucose fermentation

Alcohol dehydrogenase (NAD)

(yeast gene Adh3)

Pathway: ethanol degradation, glucose fermentation, isoleucine degradation Aldehyde dehydrogenase (NADP+) (yeast gene Ald4)
}

Cytoophidia

Punctate foci

G bodies

G bodies

G bodies

G bodies

G bodies

G bodies

G bodies

Punctate foci

Punctate foci

Punctate foci

Punctate foci

Punctate foci

Punctate foci

Foci in mitochondria

Rods in mitochondria
Stationary phase

Stationary phase (Narayanaswamy Yes et al. 2009; Saad et al. 2017), glucose starvation (Saad et al. 2017),

heat shock (Saad et al. 2017)

Hypoxia

Hypoxia

Hypoxia

Hypoxia

Hypoxia

Hypoxia

Hypoxia

Stationary phase

Stationary phase

Stationary phase

Stationary phase

Stationary phase

Stationary phase

Post-diauxic shift

Post-diauxic shift and stationary phase
No
information
provided

(Noree et al.

2010)

(Shen et al. 2016)

(Noree et al. 2019a)

(Narayanaswam-

$y$ et al. 2009)

(Saad et al. 2017)

(Jin et al. 2017)

(Noree et al.

2019a)

No information provided Yes

(Jin et al. 2017)

(Miura et al.

2013)

(Jin et al. 2017)

No

(Jin et al. 2017)

information provided

No

(Noree et al.

2019a)

information provided

No information provided

No information provided

No

information provided

(Jin et al. 2017)

(Jin et al. 2017)

(Jin et al. 2017)

(Jin et al. 2017)

Yes

(Narayanaswam$y$ et al. 2009)

Yes

(Narayanaswam$\mathrm{y}$ et al. 2009)

(Noree et al.

2019a)

Yes

(Narayanaswam-

$y$ et al. 2009)

No

(Noree et al.

information 2010)

provided

No

(Noree et al.

information 2010)

provided

No

(Noree et al.

information 2019a)

provided

No

(Noree et al.

information 2019a)

provided

(Noree et al.

2019a) 
Table 2 (continued)

Pathway: glucose fermentation

\section{No}

information

Aldehyde dehydrogenase (NADP+)

(yeast gene Ald5)

Pathway: glucose fermentation

Alditol:NADP+ 1-oxidoreductase

(yeast gene Gre3)

Pathway: xylose metabolism

Pyruvate decarboxylase

(yeast gene Pdc1)

Pathway: glucose fermentation, isoleucine degradation

Sedoheptulose-7-phosphate: D-

glyceraldehyde-3-phosphate transaldolase

(yeast gene Tal1)

Pathway: non-oxidateive branch of pentose phosphate pathway

Phosphomannomutase

(yeast gene Sec53)

Pathway: mannose biosynthesis

Triose-phosphate isomerase

(yeast gene Tpi1)

Pathway: glucose fermentation, glycolysis

Cell cycle

Mps1/Polo kinases

(Drosophila)

Pathway: cell cycle regulation

Fatty acid and sterol metabolism

Fatty acid synthase complex

(yeast genes Fas1 and Fas2)

Pathway: fatty acids and sterol metabolism

Sterol 3- $\beta$-glucosyltransferase

(yeast gene Atg26/Ugt51)

Pathway: synthesis of sterol glucoside membrane lipids

Palmitoyltransferase

(yeast gene Faa4)

Pathway: fatty acid oxidation

\section{Other}

D-Amino acid oxidase

(mammal)

Pathway: detoxification (mammals), energy production (microorganisms)

Protein kinase of the PAK/Ste20 family

(yeast gene Kic1)

Pathway: cell wall organization

Gamma-aminobutyrate (GABA) transaminase (yeast gene Uga1)

Pathway: nitrogen utilization

Serine hydroxymethyltransferase

(yeast gene Shm2)

Pathway: one-carbon metabolism

Thioredoxin peroxidase

(yeast gene Tsa1)

Pathway: cell redox homeostasis

Glutamine amidotransferase (GATase II)

(yeast gene Dug2)

Pathway: glutathione catabolic process
Foci in mitochondria

Punctate foci

Punctate foci

Punctate foci

Punctate foci

Punctate foci

Punctate foci

Punctate foci

Punctate foci

Punctate foci

Light scattering indicated polymerization

Punctate foci

Punctate foci

Punctate foci

Cytoophidia

Punctate foci
Post-diauxic shift and stationary phase

Post-diauxic shift

Stationary phase

Post-diauxic shift and stationary phase

Stationary phase

Stationary phase

Hypoxia

Glucose starvation

Stationary phase

Post-diauxic phase and log phase

Purified protein in vitro

Stationary phase

Stationary phase

Stationary phase

Stationary phase

Stationary phase
Yes

provided

No

information

provided

No

information

provided

No

information

provided

(Noree et al.

2019a)

(Noree et al.

2019a)

(Noree et al.

2019a)

No

(Noree et al.

information 2019a)

provided

No

(Noree et al.

information

provided

No

2019a)

information 2019a)

provided

Yes

(Gilliland et al.

2009)

(Pandey et al. 2007)

Yes

(Suresh et al.

2015)

(Jin et al. 2017)

(Noree,

Begovich, et

al. 2019)

(Narayanaswam-

$y$ et al. 2009)

No

information

(Noree et al.

provided

Yes

(Antonini et al.

1966)

Yes

(Narayanaswam$y$ et al. 2009)

Yes

(Narayanaswam$y$ et al. 2009)

Yes

(Narayanaswam-

$y$ et al. 2009)

(Noree et al.

2019a)

No

(Shen et al.

information 2016)

provided

No

information 2010)

provided
(Noree et al. 
Table 2 (continued)

\begin{tabular}{|c|c|c|c|c|}
\hline $\begin{array}{l}\text { Aminolevulinate dehydratase } \\
\text { (yeast gene Hem2) } \\
\text { Pathway: heme biosynthesis }\end{array}$ & Punctate foci & Stationary phase & $\begin{array}{l}\text { No } \\
\text { information } \\
\text { provided }\end{array}$ & $\begin{array}{l}\text { (Noree et al. } \\
\text { 2010) } \\
\text { (Noree et al. } \\
\quad \text { 2019a) }\end{array}$ \\
\hline $\begin{array}{l}\text { Protein of unknown function with glutathione- } \\
\text { S-transferase domain } \\
\text { (Drosophila gene Fax) }\end{array}$ & Punctate foci & Female Drosophila germ line & $\begin{array}{l}\text { No } \\
\text { information } \\
\text { provided }\end{array}$ & $\begin{array}{l}\text { (Lowe et al. } \\
\text { 2014) }\end{array}$ \\
\hline Pathway: unknown & & & & \\
\hline $\begin{array}{l}\text { Acetate-CoA ligase } \\
\text { (yeast gene Acs1) } \\
\text { Pathwav: acetate utilization, ethanol degradation }\end{array}$ & Punctate foci & Stationary phase & $\begin{array}{l}\text { No } \\
\text { information } \\
\text { provided }\end{array}$ & $\begin{array}{l}\text { (Noree et al. } \\
\text { 2019a) }\end{array}$ \\
\hline $\begin{array}{l}\text { Kynureninase } \\
\text { (yeast gene Bna5) }\end{array}$ & Rods & Stationary phase & $\begin{array}{l}\text { No } \\
\text { information }\end{array}$ & $\begin{array}{l}\text { (Noree et al. } \\
\text { 2019a) }\end{array}$ \\
\hline Pathway: de novo NAD biosynthesis & & & provided & \\
\hline $\begin{array}{l}\text { 2-(3-amino-3-carboxypropyl)histidine synthase } \\
\text { (yeast gene Dph2) } \\
\text { Pathway: Diphthamide biosynthesis }\end{array}$ & Punctate foci & Stationary phase & $\begin{array}{l}\text { No } \\
\text { information } \\
\text { provided }\end{array}$ & $\begin{array}{l}\text { (Noree et al. } \\
\text { 2019a) }\end{array}$ \\
\hline $\begin{array}{l}\text { Coproporphyrinogen III oxidase } \\
\text { (yeast gene Hem13) } \\
\text { Pathway: Heme biosynthesis }\end{array}$ & Punctate foci & Post-diauxic shift & $\begin{array}{l}\text { No } \\
\text { information } \\
\text { provided }\end{array}$ & $\begin{array}{l}\text { (Noree et al. } \\
\text { 2019a) }\end{array}$ \\
\hline $\begin{array}{l}\text { Thioredoxin-disulfide reductase } \\
\text { (yeast gene Trr1) }\end{array}$ & Punctate foci & Stationary phase & $\begin{array}{l}\text { No } \\
\text { information }\end{array}$ & $\begin{array}{l}\text { (Noree et al. } \\
\text { 2019a) }\end{array}$ \\
\hline Pathway: thioredoxin system & & & provided & \\
\hline $\begin{array}{l}\text { 2-hexaprenyl-6-methoxy-1,4-benzoquinone } \\
\text { methyltransferase } \\
\text { (yeast gene Coq5) }\end{array}$ & Punctate foci & Stationary phase & $\begin{array}{l}\text { No } \\
\text { information } \\
\text { provided }\end{array}$ & $\begin{array}{l}\text { (Noree et al. } \\
\text { 2019a) }\end{array}$ \\
\hline Pathway: ubiquinone biosynthesis & & & & \\
\hline $\begin{array}{l}\text { Fumarase } \\
\text { (yeast gene Fum1) } \\
\text { Pathway: TCA cycle, aerobic respiration }\end{array}$ & $\begin{array}{l}\text { Punctate foci in } \\
\text { mitochondria }\end{array}$ & Stationary phase & $\begin{array}{l}\text { No } \\
\text { information } \\
\text { provided }\end{array}$ & $\begin{array}{l}\text { (Noree et al. } \\
\text { 2019a) }\end{array}$ \\
\hline $\begin{array}{l}\text { Isocitrate dehydrogenase (NAD+) } \\
\text { (yeast gene Idh2) }\end{array}$ & Punctate foci & Post-diauxic shift & $\begin{array}{l}\text { No } \\
\text { information }\end{array}$ & $\begin{array}{l}\text { (Noree et al. } \\
\text { 2019a) }\end{array}$ \\
\hline Pathway: TCA cycle, aerobic respiration & & & provided & \\
\hline $\begin{array}{l}\text { Alpha-ketoglutarate dehydrogenase } \\
\text { (yeast gene Kgd1) }\end{array}$ & Foci in mitochondria & $\begin{array}{l}\text { Present in long, post-diauxic shift, } \\
\text { and stationary phase }\end{array}$ & $\begin{array}{l}\text { No } \\
\text { information }\end{array}$ & $\begin{array}{l}\text { (Noree et al. } \\
\text { 2019a) }\end{array}$ \\
\hline Pathway: TCA cycle, aerobic respiration & & & provided & \\
\hline $\begin{array}{l}\text { Dihydrolipoamide dehydrogenase } \\
\text { (yeast gene Lpd1) }\end{array}$ & Rods & Stationary phase & $\begin{array}{l}\text { No } \\
\text { information }\end{array}$ & $\begin{array}{l}\text { (Noree et al. } \\
\text { 2019a) }\end{array}$ \\
\hline Pathway: folate biosynthesis & & & provided & \\
\hline $\begin{array}{l}\text { Malate dehydrogenase (mitochondrial) } \\
\text { (yeast gene Mdh1) }\end{array}$ & Foci in mitochondria & Stationary phase & $\begin{array}{l}\text { No } \\
\text { information }\end{array}$ & $\begin{array}{l}\text { (Noree et al. } \\
\text { 2019a) }\end{array}$ \\
\hline $\begin{array}{l}\text { Pathway: TCA cycle, aerobic respiration } \\
\text { Malate dehydrogenase (cytoplasmic) } \\
\text { (yeast gene Mdh2) } \\
\text { Pathway: glyoxylate cycle and gluconeogenesis }\end{array}$ & Punctate foci & No information & $\begin{array}{l}\text { provided } \\
\text { No } \\
\text { information } \\
\text { provided }\end{array}$ & $\begin{array}{l}\text { (Noree et al. } \\
\text { 2019a) }\end{array}$ \\
\hline $\begin{array}{l}\text { Pyruvate dehydrogenase } \\
\text { (yeast gene Pda1) }\end{array}$ & Foci in mitochondria & Log phase and post-diauxic shift & $\begin{array}{l}\text { No } \\
\text { information }\end{array}$ & $\begin{array}{l}\text { (Noree et al. } \\
\text { 2019a) }\end{array}$ \\
\hline $\begin{array}{l}\text { Pathway: pyruvate dehydrogenase complex } \\
\text { Rieske iron-sulfur protein-ubiquinol- } \\
\text { cytochrome-c reductase } \\
\text { (yeast gene Rip1) }\end{array}$ & Punctate foci & Stationary phase & $\begin{array}{l}\text { provided } \\
\text { No } \\
\text { information } \\
\text { provided }\end{array}$ & $\begin{array}{l}\text { (Noree et al. } \\
\text { 2019a) }\end{array}$ \\
\hline $\begin{array}{l}\text { Pathway: aerobic respiration, electron transport } \\
\text { chain }\end{array}$ & & & & \\
\hline $\begin{array}{l}\text { Succinate dehydrogenase } \\
\text { (yeast gene Sdh4) } \\
\text { Pathway: TCA cycle, electron transport chain }\end{array}$ & Punctate foci & Stationary phase & $\begin{array}{l}\text { No } \\
\text { information } \\
\text { provided }\end{array}$ & $\begin{array}{l}\text { (Noree et al. } \\
\text { 2019a) }\end{array}$ \\
\hline
\end{tabular}

gene Prs5), which formed self-assemblies under conditions where glucose is removed from the media, suggesting inactivity in the self-assembly. The presence of colocalization was also investigated, and partial colocalization was seen with the proteins from yeast genes Asn 1 and Prs5 (asparagine and histidine/purine/pyrimidine synthesis), Bna5 and Prs5 (NAD and histidine/purine/pyrimidine synthesis), Glt1 and Prs5 (glutamate and histidine/purine/pyrimidine synthesis), Ura7 and 
Prs5 (purine and histidine/purine/pyrimidine synthesis), and Gdb1 and Ura7 (carbohydrate metabolism and purine synthesis) suggesting possible coordination of various biosynthetic pathways (Noree et al. 2019a). It was also found that the gene products of only Ade16, Ade17, and Imd3 (IMPDH) colocalize with markers of stress granules. Reversibility was investigated in some cases, and foci formed from proteins encoded by the genes Prs3, Prs5, Ade16/17, Ade12, and Imd2-4 (IMPDH) were found to disassemble in the presence of fresh glucose added to the media (or a 30-min shift to $2 \%$ glucose). Evolutionary conservation of foci/self-assembly was investigated for one of the enzymes, PRPP synthetase (yeast gene Prs5), and confirmed in Drosophila ovaries, rat hippocampal neurons, and human primary culture fibroblasts and under normal growth conditions (Noree et al. 2019a).

\section{Discussion}

\section{Filaments vary in structure}

Over 20 different enzymes are now confirmed to form linear self-assemblies, polymers, or helical filaments that have been structurally characterized by electron microscopy or x-ray crystallography (Table 1). Very likely, these structures are present in a biological context (in vivo) as well as in vitro. Another 91 more form self-assemblies in cells that resemble those formed by filament forming enzymes, but have yet to be structurally characterized in molecular detail and therefore are not yet confirmed to form filaments (Table 2). This review prioritized those self-assemblies that are known to be reversible - to distinguish from those assemblies formed by aggregates of misfolded proteins, though reversibility has not been tested in every case (see Table 2). Of those filament forming enzymes for which the structure is known at the molecular level, most form helices (about half of the known filaments are left-handed, half right-handed) and without polarity (either end is equivalent due to internal symmetry of the enzyme building block) (Table 1). Four form double helical structures (glutaminase, ribonucleotide reductase, glucokinase, and filament 3 of acetyl-CoA carboxylase, Table 1). Three form an intermediate structure composed of a ring which then stacks linearly to create a filament (glutamine synthetase, $\beta$-glucosidase, and 2-cys peroxiredoxin). Adenylate kinase and casein kinase 2 form linear filaments, without any helical nature. The death domain (DD) containing proteins can form complicated filaments with different proteins assembling in a specific order. Finally, in some cases, the filaments have a large internal cavity and form hollow tubes (TORC1, $\beta$-glucosidase, nitrilase, glutamate dehydrogenase, and 2-cys peroxiredoxin). Hence, filaments form in a wide array of structures.
Although we have made an effort to collate a comprehensive list of proteins found to form filaments or at least cellular self-assemblies, we have not included the additional proteins uncovered in screens of protein aggregation in response to heat stress or arsenic, because our interest is in enzyme regulation and these screens likely identify proteins undergoing irreversible aggregation due to misfolding. Although it should also be noted that many of those proteins found to form cellular self-assemblies under nutrient stress also are found in the heat shock (or arsenic) induced cellular aggregates as well (Jacobson et al. 2012; Ibstedt et al. 2014; O'Connell et al. 2014). We have also not covered the proteins found in a screen for prion-like assembly formation (Alberti et al. 2009), as these protein assemblies have several distinct biophysical features affecting their solubility, reversibility, and heritability. Further, many other proteins and enzymes can be found in RNA-rich self-assemblies such as P-bodies, stress granules, and other membraneless compartments that are not given in Table 2, with filament formation yet to be investigated (Aulas and Vande Velde 2015; Jain et al. 2016; Saarikangas and Barral 2016).

\section{Effect of filamentation on enzyme activity}

Perhaps counterintuitively, most of the enzymes known to be modulated in activity by filament formation are activated in the filamentous state (13 total including filaments 1 and 2 of ACC, human CTPS, IMPDH, $\beta$-glucosidase, $\mathrm{CO}_{2}$-reductase, nitrilase, glutaminase, SgrAI, IRE1, RecA, DnaA, RIP1/3 kinases, and death domain containing, Table 1). Six enzymes are known to be inhibited (ACC filament 3, glucokinase, bacterial CTPS, glutamine synthetase, ribonucleotide reductase, and TORC1, Table 1). IMPDH is also suspected of having an inactive filamentous state. The enzyme 2-cys peroxiredoxin shifts from peroxireductase activity in the non-filamentous state, to a chaperone in the filament form which is inactive in peroxireductase activity. Hence, it is inactive in the dimeric state, but gains an entirely new activity in the filamentous state. In the case of LACTB, which has sequence homology with $\beta$-lactamases, it is not actually known if it possesses any enzymatic activity, much less how such activity is modulated by filamentation. Bacterial CTPS is thought to possibly act in cell shape determination when filamented (Barry and Gitai 2011). It is not yet known if PFK, AdhE, and glutamate dehydrogenase are activated in the filament form, but other considerations suggest that they may be activated because the filaments form under conditions of high demand in the cell or high concentrations of substrate (PFK (Jang et al. 2016, Webb et al. 2017)), filaments form under conditions where the enzyme is more active (glutamate dehydrogenase (Frieden 1959b, Gylfe 1976, Fahien et al. 1989)), or have active sites more accessible to substrates (AdhE (Kessler et al. 1992)) (Table 1). CK2 and adenylate kinase may be 
inhibited in the filamentous state (Table 1). For enzymes of Table 2, it is not known how their activity is affected by filament formation, but many form filaments under nutrient starvation conditions suggesting that they filament in order to store inactivated enzymes until needed. Alternatively, filamentation may activate enzymes under conditions of stress due to high demand (such as G-bodies composed of enolase, PFK, and/or fructose bisphosphate aldolase) (Jin et al. 2017).

\section{Filamentation can change enzyme specificity}

In only a few enzymes does the substrate specificity change when in the filament form. SgrAI, a sequence specific endonuclease, cleaves 17 different 8 bp DNA sequences in the filamentous site, but only 3 in the non-filamentous state (Bitinaite and Schildkraut 2002; Park et al. 2010; Shah et al. 2015). IRE1 may also have expanded substrate specificity when filamentous, cleaving other RNAs in addition to its canonical RNA substrate (Tam et al. 2014). The enzyme 2-cys peroxiredoxin changes specificity and enzymatic activity when filamentous, changing from a peroxireductase to a chaperone (Noichri et al. 2015; Teixeira et al. 2019). A change in specificity to smaller substrates, due to reduced access to active sites located within the central tunnel of a fenestrated tube, was suggested for $\beta$-glucosidase (Kim et al. 2005). Nitrilases from different organisms assemble into filaments with different helical twists, which correlate with their exhibited substrate specificities (Woodward et al. 2018). Hence, although not changing within the same filament, the family of nitrilase enzymes show that filaments may evolve to fine-tune activity and specificity for particular cellular needs.

\section{Regulation of filament formation}

Regulation of filament formation also varies. Enzyme filaments stimulated by their substrates (and which become activated) include human CTPS, nitrilases, SgrAI (which is also stimulated by its product), RecA, and DnaA (Table 1). In the case of PFK, it is not known but likely to be activated in the filament, because filament formation is stimulated by its substrate (Webb et al. 2017). Filaments of glucokinase form in response to substrates ATP and glucose (Stoddard et al. 2019), when the enzyme concentration is above a critical concentration $(2 \mu \mathrm{M})$, but is inactivated in the filamentous form. 2-Cys peroxiredoxin is stimulated by its substrate, $\mathrm{H}_{2} \mathrm{O}_{2}$, to form filaments with a new activity (binding unfolded proteins) but which is inactive in peroxiredoxin activity (Angelucci et al. 2013). Filaments forming in response to their enzymatic product include SgrAI (cleavage products of their primary recognition sites which stimulate the enzyme activity), glucokinase (inhibited in the filament), and bacterial CTPS (also inhibited as a consequence of filamentation) (Table 1). Many enzyme filaments form in response to another signal, including binding to allosteric effectors (PFK, ACC filaments 1-2, IMPDH, ribonucleotide reductase and glutamate dehydrogenase), or ligands $\left(\mathrm{CO}_{2}\right.$ reductase, IRE1 and many death domain containing proteins) (Table 1). At least two enzymes are known to form filaments as a result of phosphorylation (ACC filament 3 and 2-cys peroxiredoxin), one from protein binding (ACC filament 3), and others in response to buffer components (nitrilase, glutaminase, glutamine synthetase, $\mathrm{CO}_{2}$ reductase, 2-cys peroxiredoxin) (Table 1). Phosphorylation stabilizes IRE1 filaments, as does ADP binding (Korennykh et al. 2009). Just as filaments are stimulated in response to environmental stimuli, they may also be induced to disassemble. Those disassembled by their products, direct or downstream, include glutamate dehydrogenase (Huang and Frieden 1972; Zeiri and Reisler 1978) and human CTPS (Lynch et al. 2017). Filament formation by ACC1 and ACC2 are reportedly inhibited by binding to SPOT14 and MIG12 proteins (Park et al. 2013) (although another report suggests MIG12 stimulates filament formation (Kim et al. 2010)). Others are known to be disassembled by buffer components ( $\mathrm{pH}$ for $\mathrm{Gln} 1, \mathrm{CaCl}_{2}$ for $\beta$-glucosidase (Gunning 1965, Nisius and Ruppel 1987), and high ionic strength for casein kinase (Valero et al. 1995)).

\section{Direct transformation from one type of filament to another}

Some filamentous enzymes may be capable of responding to environmental conditions without dissociating and reassembling into a new filamentous structure. Instead, these remain filamentous but coordinately (or not) change conformation to active or inactive enzymatic activity. Three possible examples of this have been described. First, ACC forms the filament 1 type filament in the presence of citrate and when the enzyme is not phosphorylated. The addition of a product of a reaction downstream of ACC, palmitoyl-CoA, results in what appears to be an altered filament 1 , but which now is inactive, and is named filament 2 (Hunkeler et al. 2018). However, it is not known for certain at this time if this transformation from the active filament 1 form to the inactive filament 2 form occurs without dissociating into ACC protomers. The second example is IMPDH. ATP stabilizes both types of filaments which are composed of stacked octameric IMPDH enzymes. The octameric IMPDH enzyme has the appearance of a hollow sphere with openings on the sides presumably for substrate and product diffusion. The open form is stabilized in the presence of a cofactor for the reaction, $\mathrm{NAD}^{+}$, while the closed form is stabilized by a downstream product of the enzyme pathway, GTP. The closed form is likely inactive, due to lack of substrate access to the active site, while the open form is likely the active state. Both states, open and closed octamers, have been visualized within the same filament (Anthony et al. 2017). This may indicate less cooperativity 
in the filament, since both states coexist, but it also indicates that the filament can be sensitive and responsive to molecular signals without dissociating. The third example is AdhE. Upon addition of cofactors $\mathrm{Fe}^{2+}$ and $\mathrm{NAD}^{+}$, the filaments appear slimmer and more extended (Kessler et al. 1992). This indicates responsiveness of the filament to the environment, although transition from one filamentous form to the other was not strictly investigated, hence these two forms may have required dissociation and reassociation. Further investigations are needed.

\section{Enzymes that form more than one type of filament}

Five enzymes were shown to form more than one type of filament. Two were discussed above, ACC and AdhE, and in addition, $\mathrm{ACC}$ forms a third type of filament that requires phosphorylation of the enzyme and binding of the BRCT domains of BRCA1 (Hunkeler et al. 2018). Further, ACC may also form yet additional filament types, as filamentation in vitro and in vivo has been shown to be stimulated by the protein MIG12, which appears to form a co-filament with ACC (Kim et al. 2010). Ribonucleotide reductase also forms different filamentous forms, both inactive, but which differ in architecture (one is a double helix, the other is a single helix) and the presence or absence of a protein $\mathrm{NrdF}$ (Thomas et al. 2019). CTPS also forms two types of filaments, one active and one inactive. Although in this case it is not the same CTPS enzyme; instead it is CTPS from a bacterial source that forms an inactive enzyme filament (Noree et al. 2014), and CTPS from human that forms an active enzyme filament (Lynch et al. 2017). The filaments have similarities in structure, in that they are formed by stacking the tetrameric CTPS, but differences in the conformation of the tetramer and hence also the interfaces between tetramers within the filaments. These two filaments appear to be representatives of the type of activation or inhibition that derives from stabilization of either the active or inactive conformation of the enzyme by contacts between adjacent enzymes within the filament. Finally, nitrilases from different organisms have been examined and found to differ in the helical twist between the enzyme protomers, as mentioned above (Woodward et al. 2018). Interestingly, this difference in twist correlates with substrate preference, and hence evolution may have modified the filament structure to fine tune enzymatic specificity.

\section{Morpheins}

Some filament forming enzymes are also morpheins (Selwood and Jaffe 2012). Morpheins are proteins which can form more than one type of oligomer, each having distinct enzymatic properties, and where conversion from one type to the other requires dissociation into a more fundamental building block under normal physiological conditions. The interconversion is also post-translational modification independent. The shifting between oligomeric states is controlled by binding to allosteric effectors. The classic example of a morpheein is porphobilinogen synthase (Breinig et al. 2003; Tang et al. 2005; Tang et al. 2006; Selwood et al. 2008). As for filament forming enzymes, the filament being one oligomeric state, 2cys peroxiredoxin and ribonucleotide reductase fit the strict requirements of a morpheein. Other enzymes, such as SgrAI, are related to the morpheein model in that a fundamental building block (the SgrAI dimer) is stimulated to form a different, more active oligomeric form (a filament). However, it is stimulated by is substrate and product, rather than a distinct allosteric effector. It also does not have a second oligomeric state beyond the fundamental building block.

\section{Biological roles and possible selective advantages of filament formation by enzymes}

It is clear now that many enzymes form filaments and large self-assemblies, and for some, the role and advantage of those structures are yet to be uncovered. Many have stimulated enzymatic activity in these self-assemblies, yet others are reduced or inhibited, showing filament formation to be another layer of enzyme regulation. Why filaments and large selfassemblies are necessary rather than (or in addition to) other mechanisms is an important next question for researchers. It may be that formation of the filament enables more rapid, and more cooperative activation or inactivation of the enzyme, as has been suggested for SgrAI (rapid activation (Barahona et al. 2019)) and E. coli CTPS (rapid inactivation (Barry et al. 2014)). Many enzymes regulated by filament formation control key steps in biochemical pathways, such as PFK, which may require the additional layer of regulation (Webb et al. 2017). Why a filament and not a discrete oligomer, such as a dimer, tetramer, and hexamer is formed may be due to the fact that evolving a filament requires the selection of changes that result in a single type of interface between protomers that can propagate without steric hindrance, vs. a discrete oligomer such as a dimer that would require evolving an interface with twofold symmetry that occurs only once between protomers (Garcia-Seisdedos et al. 2017). Evolving other discrete oligomers would require such interfaces to lead to a ring, to "close the ends." Interestingly, such closed ring oligomers can themselves form filaments by stacking, as discussed above.

Various strategies for controlling enzyme activity by filament formation are found. One strategy is to evolve an interface between protomers that preferentially stabilizes one conformation of the enzyme, either the active or the inactive state. Hence, the filamented enzyme is locked in one conformation and cannot access the other state. Such is proposed for several enzymes, including SgrAI, glucokinase, and the CTPS enzymes. In the ribonucleotide reductase filament, binding of a second protein $(\mathrm{NrdF})$ to the filament formed by NrdE leads to 
an inter-subunit gap that is too large to allow for radical transfer, thereby inactivating the enzyme (Thomas et al. 2019). In adenylate kinase, the active site is blocked by the filament structure (Wild et al. 1997). Another strategy, for activating an enzyme by filamentation, is to create active sites from multiple enzyme protomers within the filament, such as filament 1 of ACC (Hunkeler et al. 2018), DnaA (Duderstadt et al. 2011), and RecA (Chen et al. 2008a). Alternatively, formation of an internal tunnel within a hollow filament can aid in channeling substrates and products to different active sites as proposed in AdhE (Extance et al. 2013), and may be a central feature of G-bodies, purinosomes, and other metabolically related structures. The internally located active sites in hollow filaments means that substrates and products must diffuse through gaps between protomers, which may allow for mechanisms of selectively of substrates, as suggested for nitrilases (Thuku et al. 2009) and $\beta$-glucosidase (Kim et al. 2005). In addition, forming a large assembly in the cell could localize products of the enzymatic reaction to particular locations. This was suggested for several enzymes activated by filament formation, but also $\beta$-glucosidase, where the filamentous structures produce anti-fungals and are activated upon infection (Kim et al. 2005). Conversely, filament formation may sequester enzymes away from one another and their substrates, modulating metabolic flux.

The filaments may be necessary to bind large substrates, as in the case of DnaA, RecA, IRE1, nitrilases, and significantly, for altering substrate specificities, as in IRE1 which cleaves additional mRNAs using an activity known as RIDD upon filamentation, and SgrAI, which cleaves an additional 14 DNA sequences when in the filament form. Significantly, simulations of enzyme activity based on kinetic modeling indicate that the filamentation mechanism can act to sequester enzymatic activity on only a subset of available substrates (Park et al. 2018a; Park et al. 2018b; Barahona et al. 2019). For example, as a direct consequence of filament formation, the expansion of substrate specificity by SgrAI occurs only on DNA molecules containing the SgrAI primary recognition sequence. The filament takes advantage of the higher local concentration of different recognition sites present on the same molecule of DNA to induce filamentation and cleavage activity on the secondary site sequences on the same contiguous molecule of DNA. Though this could be accomplished by a finite, discrete oligomer, the filament has the advantage of also maximizing speed of enzyme activation as well (Barahona et al. 2019). Local concentration effects are easily possible on large multi-substrate molecules such as DNA, but such activity may also be possible wherever high concentrations of a preferred substrate occur, which can induce filament formation to act upon a second type of substrate. Hence, the second type of substrate is acted upon only where the high local concentrations of the preferred substrate occurs (Fig. 31), which could result from the activity of nearby enzymes, or in particular compartments including phase separated assemblies (liquid separated droplets in cells often formed from RNA and proteins) (Prouteau and Loewith 2018).

Some filaments appear capable of switching between active and inactive conformations in the same filament, such as ACC, AdhE, and IMPDH. The purpose of the filament may be for more rapid communication of a signal for activation or alternatively, for inactivation. In this case, it is akin to a multimeric enzyme with a large degree of cooperativity.

The filaments and self-assemblies may be a way to store unneeded enzymes, to protect against degradation, and to be rapidly redeployed upon, for example, reintroduction of nutrients to the environment without the need for new transcription and/or translation. Such has been suggested for the cytoophidia and foci formed upon nutrient starvation (Narayanaswamy et al. 2009; Petrovska et al. 2014). Filament formation may also be used to protect active enzymes from degradation or oxidation, as has been suggested for $\mathrm{CO}_{2}$ reductase (Schuchmann et al. 2016) and AdhE (Extance et al. 2013).

Buffering of enzymatic activity is another proposed role of enzyme filaments and self-assemblies. Active enzymes in the non-filamentous state can reach an equilibrium with the inactive, filamentous state and by modulation of the on and off rates of this equilibrium, different levels of active enzyme in the pool can be achieved, as proposed for bacterial CTPS (Aughey et al. 2014). Thus, the level of active enzyme in a cell can be easily maintained and adjusted as needed. In the case of glucokinase, active monomeric enzymes reach an equilibrium with those in the filaments (composed of inactivated glucokinase), thereby maintaining a constant concentration of active enzyme and creating an upper limit to the maximum enzyme activity. This protects the cells from sudden "surges" in glucokinase activity that could be toxic. Binding of proteins to the filament to stabilize it from dissociation, such as filament 3 of ACC with the binding of BRCT1 (Hunkeler et al. 2018), could further regulate filament dissociation.

Another reason for filament formation by enzymes may be to control water activity. Formation of large complexes such as enzyme filaments reduces the available protein surface area, thereby significantly reducing the amount of water required for full hydration of the protein (Goodsell and Olson 2000). Trehalsoe-6-phosphate synthase complex forms selfassemblies in cells in response to desiccation (Jin et al. 2017). Adenylate kinase, found in plants, is thought to form filaments at night when the enzyme is not needed, to free up available water (Wild et al. 1997).

Several enzymes gain additional functionality in the filament form. The enzyme 2-cys peroxiredoxin is a striking example which switches from a peroxiredoxin to a chaperone upon oxidative stress (Wood et al. 2002). The enzyme functions to eliminate $\mathrm{H}_{2} \mathrm{O}_{2}$ at lower levels, but higher levels will induce greater oxidation and eventually the formation of a 


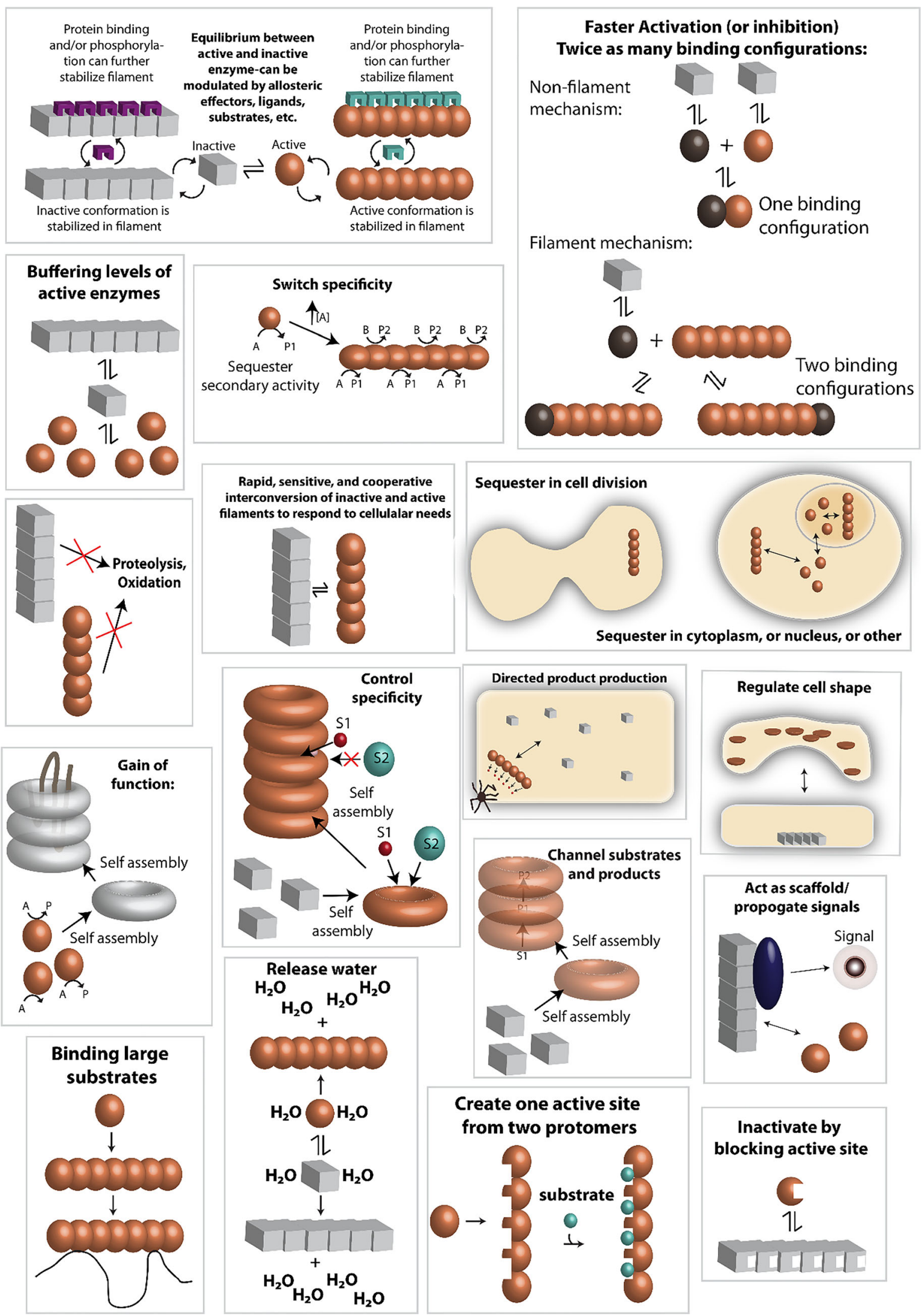

Fig. 31 Biological roles of filament forming enzymes. Grey squares, inactive enzyme; copper spheres, active enzyme 
hollow filament capable of binding unfolded proteins in its interior. This type of chaperone activity is non-ATP dependent and termed "holdase" since it sequesters the unfolded proteins from one another to prevent their aggregation. CTPS from C. crescentas is another example, which regulates cell shape in its filamentous form (Ingerson-Mahar et al. 2010). Acting as a scaffold for the binding of other proteins and enzymes is another gain of function that has been suggested for many filament forming enzymes. Certainly, the death domain (DD) containing proteins and RIP1/RIP3 kinases do this, allowing binding and activation of downstream effector enzymes such as kinases, proteases, and membrane pore forming proteins (Cho et al. 2009; Sun et al. 2012; Zhao et al. 2012; Lu et al. 2014a; Lu and Wu 2015). Relatedly, the filaments could be a signal themselves to the cell of the levels of metabolites or other environmental stimuli. In addition to the proteins and enzymes of innate immunity (DD and RIP1/RIP3 kinases), this function has been proposed for IMPDH (Anthony et al. 2017) and the kinases Mps1 and Polo (Gilliland et al. 2009). In addition, it was suggested for IRE1 that filament formation could prolong the signal induced by unfolded proteins since self-assembly is self-propagating and stabilizing via autophosphoryation (Korennykh et al. 2009), similar to inflammasomes composed of RIP1/RIP3 kinases and also DD enzymes like RIP2 (Li et al. 2012a; Gong et al. 2018).

Finally, there may be a role for filamentation in sequestering enzymes to particular compartments, such as cytoplasm, nucleus, or the mother cell in dividing cells. This was proposed for IMPDH, since in Drosophila, it acts also as a transcription factor (Kozhevnikova et al. 2012). Thayer and coworkers proposed sequestration upon cell division for homocysteine kinase (Thayer et al. 2014).

\section{Unique features of SgrAI}

The most defining and unusual characteristic of the SgrAI endonuclease is its ability to use filament formation to modulate its DNA sequence specificity in a very targeted way. It does this by using the DNA sequence of its recognition sequence to modulate the equilibrium between two conformational states, an inactive, non-filament forming state, and an activated, filament forming state(Polley et al. 2019). Binding to its primary recognition sequences shifts this equilibrium to the active, filament forming state more than does binding to its secondary site sequence. Hence, it is SgrAI bound to primary sites that will drive filament formation, although SgrAI bound to secondary site sequences will join filaments under particular conditions. Joining the filament leads to activation of SgrAI and rapid cleavage of the bound DNA. However, joining is limited by a very slow association rate constant (Park et al. 2018a). To overcome this limitation, high concentrations of filament forming states are necessary. In the cell, this is accomplished by SgrAI binding to primary recognition sequences on the same DNA molecule, and the high local concentration therefore drives the association of these SgrAI into a filament. Primary sites on the host are methylated and will not be bound by SgrAI, but invading DNA is not expected to have the same protection. Hence, unmethylated primary sites on the phage DNA drive SgrAI to form filaments, which also recruit SgrAI bound to secondary site sequences on the same DNA. Secondary sites are not methylated on the host and therefore are at risk of being cleaved by activated SgrAI. SgrAI binds to secondary sites but does not cleave them unless activated by recruitment into a filament. However, the slow association rate constant for their incorporation into the filament, and the low concentration of DNA in the cell, prevents their recruitment into the filament formed on the invading DNA. Only the high local concentration of SgrAI bound to secondary sites on the invading DNA can drive their incorporation into filaments formed by SgrAI bound to primary sites there (Barahona et al. 2019).

The computational model developed from the kinetic study of DNA cleavage by SgrAI also found that the filament mechanism imparted greater speed in product formation, and with a greater ability to sequester its activity (between sites on invading and host DNA) (Barahona et al. 2019). The sequestration of activity derives from the slow association rate constant for filament formation. Association and dissociation rates of enzymes into and out of filaments to our knowledge have not been measured, with the exception of the observation in some limited cases of cytoophidia dynamics. It remains then to be seen how general or specific this particular but distinguishing property of the SgrAI system is. As for the property of rapid activation, the opposite, rapid, and efficient inactivation has been suggested for the bacterial CTPS system. A mutation in CTPS, E155K, eliminates the ability to form filaments, but also does not show the significant reduction in activity shown by wild type CTPS (Trudel et al. 1984; Barry et al. 2014).

Some other unique features of the SgrAI system are also found, including filament formation being stimulated by both substrate and product (of the primary site sequence), participation of the substrate (and product) in stabilization of the filament via direct interactions with neighboring protomers, and the requirement to dissociate from the filament in order to release product (Park et al. 2010; Lyumkis et al. 2013; Shah et al. 2015; Park et al. 2018a; Park et al. 2018b; Barahona et al. 2019). We found that the dynamics of SgrAI enzymes into and out of the filament is sufficiently fast to prevent trapping of the product, despite this requirement. Many filamentous enzymes with smaller substrates and products would not have such barriers to product dissociation.

Only IRE1 may share the unique property of substrate specificity modulation via filament formation, since some evidence suggests that the filamentous form is more effective in RIDD, an activity which results in degradation of other 
mRNAs in addition to that which is spliced by IRE1 (Kimmig et al. 2012). SgrAI cleaves 14 additional (secondary site) DNA sequences in the filamentous state, but only 3 (primary sites) in the non-filamentous state. It does so by drawing SgrAI bound to secondary site DNA sequences into filaments formed by SgrAI bound to primary site, thereby activating SgrAI to cleave the secondary sites. Secondary sites will not induce SgrAI to form filaments. A somewhat similar situation is found with $\beta$-glucosidase, two isomeric forms of the enzyme are known, As-Glul and As-Glu2. As-Glu2 forms a homodimer, but will be drawn into filaments formed by As-Glu1 (Kim et al. 2000). However, both forms of the enzyme as well as both states (filament and non-filamentous) possess the same enzyme specificity.

As for the qualities of becoming activated in the filamentous state, 13 or more enzymes share this property; however, quantification of exactly how much more active is lacking for most systems. For SgrAI, the observed rate constants for cleavage of primary DNA show a 200-fold increase under filament forming conditions compared to non-filament forming conditions, and for secondary site sequences, this number is 1000-fold (Shah et al. 2015). However, kinetic modeling found the rate constant for activated DNA cleavage in the filamentous state is 0.8 $\mathrm{s}^{-1}, 480$ times faster than the observed DNA cleavage rate constant of primary sites in the non-filamentous state, and nearly 4000 times faster than with secondary sites (Shah et al. 2015; Park et al. 2018b). Finally, in terms of timescale, our studies show association/dissociation of SgrAI with rate constants of $3 \times 10^{5} \mathrm{M}^{-1} \mathrm{~s}^{-1}$ and $0.02 \mathrm{~s}^{-1}$, resulting in very fast (subsecond) association under optimal conditions and a half-life of the complex on the order of seconds (Park et al. 2018b). The timescale of filaments and cellular assemblies of other enzymes is less well characterized, though studies of Mps1/Polo kinases show complete dissociation after $30 \mathrm{~min}$, and complete refilamentation upon reintroduction of filament inducing conditions after another $30 \mathrm{~min}$ (Gilliland et al. 2009).

\section{Relationship between molecular filament formation and foci/condensates/self-assemblies in cells}

Finally, the issue of length and time scales of filament forming enzymes requires addressing. Table 1 summarizes enzymes where the molecular structure of the filamentous state is known, but in many cases, it is also known that these enzymes form large assemblies in cells that must be composed of either assemblies of these filaments, or perhaps some other structure. Table 2 summarizes enzymes for which only the cellular assembly (i.e., foci or condensate) has been observed, but studies of the molecular details of the assemblies have not yet been performed; hence, it is unknown if they form filaments at the nanoscale. In one case, electron microscopy of a cell showed the large assembly to be composed of layers of aligned filaments. One could imagine that this would be most efficient for the purposes of storing and protecting inactive enzymes. In other cases, the assemblies may have other geometries, perhaps less organized (networks) and more like gels (Prouteau and Loewith 2018) (PFK, known to form filaments, also forms liquid-like droplets in cells (Jang et al. 2019)). This may allow substrates and products to diffuse in and out of the large-scale assembly/droplet, perhaps making use of local concentration to alter enzyme specificity, as in the SgrAI case, but using phase separation to induce the high local concentrations.

\section{Conclusions}

In conclusion, nearly 116 enzymes have been shown to form some sort of self-assembly either in cells or in vitro. Of these, 24 have been characterized for their assembly structure. Structures vary from the less common linear filament and ring stacks, to more common helical filaments, either right or left-handed. Enzyme activity may be enhanced in the filament, or inhibited, and less commonly, altered in some form. Some enzymes form more than one type of filament each with unique enzyme activities. Beyond controlling enzyme activity, other functions such as scaffolding, controlling cell shape, and signaling have also been shown. In the case of SgrAI, a type II restriction endonuclease, studies show that filament formation provides a clever means to control DNA cleavage behavior onto only invading DNA. The existence of filaments in vivo, their regulation and activity, and the wide range of diversity in structure and complexity is an emerging phenomenon in our biophysical understanding of cell biology. The relationship between the molecular filament structure at the nanoscale and the cellular self-assembly at the microscale remains largely unknown for the vast majority of enzymes. In the coming years, as more studies on filament forming enzymes are performed, no doubt new and interesting purposes and advantages will be uncovered for enzyme filamentation and cellular self-assembly.

Funding information This work was supported by the National Science Foundation under Grant No. MCB-1410355 (to N.H.).

Open Access This article is distributed under the terms of the Creative Commons Attribution 4.0 International License (http:// creativecommons.org/licenses/by/4.0/), which permits unrestricted use, distribution, and reproduction in any medium, provided you give appropriate credit to the original author(s) and the source, provide a link to the Creative Commons license, and indicate if changes were made. 


\section{References}

Abu-Elheiga L, Jayakumar A, Baldini A, Chirala SS, Wakil SJ (1995) Human acetyl-CoA carboxylase: characterization, molecular cloning, and evidence for two isoforms. Proc Natl Acad Sci U S A 92(9): 4011-4015

Abu-Elheiga L, Almarza-Ortega DB, Baldini A, Wakil SJ (1997) Human acetyl-CoA carboxylase 2. Molecular cloning, characterization, chromosomal mapping, and evidence for two isoforms. J Biol Chem 272(16):10669-10677

Abu-Elheiga L, Matzuk MM, Abo-Hashema KA, Wakil SJ (2001) Continuous fatty acid oxidation and reduced fat storage in mice lacking acetyl-CoA carboxylase 2. Science 291(5513):2613-2616

Abu-Elheiga L, Oh W, Kordari P, Wakil SJ (2003) Acetyl-CoA carboxylase 2 mutant mice are protected against obesity and diabetes induced by high-fat/high-carbohydrate diets. Proc Natl Acad Sci U S A 100(18):10207-10212

Alberti S, Halfmann R, King O, Kapila A, Lindquist S (2009) A systematic survey identifies prions and illuminates sequence features of prionogenic proteins. Cell 137(1):146-158

Ali MM, Bagratuni T, Davenport EL, Nowak PR, Silva-Santisteban MC, Hardcastle A, McAndrews C, Rowlands MG, Morgan GJ, Aherne W, Collins I, Davies FE, Pearl LH (2011) Structure of the Ire1 autophosphorylation complex and implications for the unfolded protein response. EMBO J 30(5):894-905

An S, Kumar R, Sheets ED, Benkovic SJ (2008) Reversible compartmentalization of de novo purine biosynthetic complexes in living cells. Science 320(5872):103-106

Ando N, Li H, Brignole EJ, Thompson S, McLaughlin MI, Page JE, Asturias FJ, Stubbe J, Drennan CL (2016) Allosteric Inhibition of human ribonucleotide reductase by dATP entails the stabilization of a hexamer. Biochemistry 55(2):373-381

Angelucci F, Saccoccia F, Ardini M, Boumis G, Brunori M, Di Leandro L, Ippoliti R, Miele AE, Natoli G, Scotti S, Bellelli A (2013) Switching between the alternative structures and functions of a 2Cys peroxiredoxin, by site-directed mutagenesis. J Mol Biol 425(22):4556-4568

Angermuller S, Bruder G, Volkl A, Wesch H, Fahimi HD (1987) Localization of xanthine oxidase in crystalline cores of peroxisomes. A cytochemical and biochemical study. Eur J Cell Biol 45(1):137144

Anthony SA, Burrell AL, Johnson MC, Duong-Ly KC, Kuo YM, Simonet JC, Michener P, Andrews A, Kollman JM, Peterson JR (2017) Reconstituted IMPDH polymers accommodate both catalytically active and inactive conformations. Mol Biol Cell

Antonini E, Brunori M, Bruzzesi R, Chiancone E, Massey V (1966) Association-dissociation phenomena of D-amino acid oxidase. J Biol Chem 241(10):2358-2366

Aragon T, van Anken E, Pincus D, Serafimova IM, Korennykh AV, Rubio CA, Walter P (2009) Messenger RNA targeting to endoplasmic reticulum stress signalling sites. Nature 457(7230):736-740

Ashcraft BA, Fillers WS, Augustine SL, Clarke SD (1980) Polymerprotomer transition of acetyl-CoA carboxylase occurs in vivo and varies with nutritional conditions. J Biol Chem 255(21):1003310035

Atkin JD, Farg MA, Walker AK, McLean C, Tomas D, Horne MK (2008) Endoplasmic reticulum stress and induction of the unfolded protein response in human sporadic amyotrophic lateral sclerosis. Neurobiol Dis 30(3):400-407

Aughey GN, Grice SJ, Shen QJ, Xu Y, Chang CC, Azzam G, Wang PY, Freeman-Mills L, Pai LM, Sung LY, Yan J, Liu JL (2014) Nucleotide synthesis is regulated by cytoophidium formation during neurodevelopment and adaptive metabolism. Biol Open 3(11): $1045-1056$
Aulas A, Vande Velde C (2015) Alterations in stress granule dynamics driven by TDP-43 and FUS: a link to pathological inclusions in ALS? Front Cell Neurosci 9

Aylett CH, Lowe J, Amos LA (2011) New insights into the mechanisms of cytomotive actin and tubulin filaments. Int Rev Cell Mol Biol 292:1-71

Bailey J, Bell ET, Bell JE (1982) Regulation of bovine glutamate dehydrogenase. The effects of pH and ADP. J Biol Chem 257(10):55795583

Barahona CJ, Basantes LE, Tompkins KJ, Heitman DM, Chukwu BI, Sanchez J, Sanchez JL, Ghadirian N, Park CK, Horton NC (2019) The need for speed: run-on oligomer filament formation provides maximum speed with maximum sequestration of activity. J Virol 93(5):1-19

Barry RM, Gitai Z (2011) Self-assembling enzymes and the origins of the cytoskeleton. Curr Opin Microbiol 14(6):704-711

Barry RM, Bitbol AF, Lorestani A, Charles EJ, Habrian CH, Hansen JM, Li HJ, Baldwin EP, Wingreen NS, Kollman JM, Gitai Z (2014) Large-scale filament formation inhibits the activity of CTP synthetase. Elife 3:e03638

Beaty NB, Lane MD (1983) Kinetics of activation of acetyl-CoA carboxylase by citrate. Relationship to the rate of polymerization of the enzyme. J Biol Chem 258(21):13043-13050

Bernstein BW, Bamburg JR (2010) ADF/cofilin: a functional node in cell biology. Trends Cell Biol 20(4):187-195

Bernstein BW, Chen H, Boyle JA, Bamburg JR (2006) Formation of actin-ADF/cofilin rods transiently retards decline of mitochondrial potential and ATP in stressed neurons. Am J Phys Cell Phys 291(5): C828-C839

Bershadsky AD, Vasil'ev IUM (1988) Cytoskeleton. Plenum Press, New York

Bilcock DT, Daniels LE, Bath AJ, Halford SE (1999) Reactions of type II restriction endonucleases with 8-base pair recognition sites. J Biol Chem 274(51):36379-36386

Bitinaite J, Schildkraut I (2002) Self-generated DNA termini relax the specificity of SgrAI restriction endonuclease. Proc Natl Acad Sci U S A 99(3):1164-1169

Bleichert F, Botchan MR, Berger JM (2017) Mechanisms for initiating cellular DNA replication. Science 355(6327)

Breinig S, Kervinen J, Stith L, Wasson AS, Fairman R, Wlodawer A, Zdanov A, Jaffe EK (2003) Control of tetrapyrrole biosynthesis by alternate quaternary forms of porphobilinogen synthase. Nat Struct Biol 10(9):757-763

Brownsey RW, Boone AN, Elliott JE, Kulpa JE, Lee WM (2006) Regulation of acetyl-CoA carboxylase. Biochem Soc Trans 34(Pt 2):223-227

Bruser A, Kirchberger J, Kloos M, Strater N, Schoneberg T (2012) Functional linkage of adenine nucleotide binding sites in mammalian muscle 6-phosphofructokinase. J Biol Chem 287(21):1754617553

Cabeen MT, Jacobs-Wagner C (2010) A metabolic assembly line in bacteria. Nat Cell Biol 12(8):731-733

Calfon M, Zeng H, Urano F, Till JH, Hubbard SR, Harding HP, Clark SG, Ron D (2002) IRE1 couples endoplasmic reticulum load to secretory capacity by processing the XBP-1 mRNA. Nature 415(6867):92-96

Calise SJ, Carcamo WC, Krueger C, Yin JD, Purich DL, Chan EK (2014) Glutamine deprivation initiates reversible assembly of mammalian rods and rings. Cell Mol Life Sci 71(15):2963-2973

Campbell SG, Hoyle NP, Ashe MP (2005) Dynamic cycling of eIF2 through a large eIF2B-containing cytoplasmic body: implications for translation control. J Cell Biol 170(6):925-934

Carcamo WC, Satoh M, Kasahara H, Terada N, Hamazaki T, Chan JY, Yao B, Tamayo S, Covini G, von Muhlen CA, Chan EK (2011) Induction of cytoplasmic rods and rings structures by inhibition of the CTP and GTP synthetic pathway in mammalian cells. PLoS One 6(12):e29690 
Carcamo WC, Calise SJ, von Muhlen CA, Satoh M, Chan EK (2014) Molecular cell biology and immunobiology of mammalian rod/ring structures. Int Rev Cell Mol Biol 308:35-74

Carr SF, Papp E, Wu JC, Natsumeda Y (1993) Characterization of human type I and type II IMP dehydrogenases. J Biol Chem 268(36): 27286-27290

Cassman M, Schachman HK (1971) Sedimentation equilibrium studies on glutamic dehydrogenase. Biochemistry 10(6):1015-1024

Chan KY, Gumbart J, McGreevy R, Watermeyer JM, Sewell BT, Schulten K (2011) Symmetry-restrained flexible fitting for symmetric EM maps. Structure 19(9):1211-1218

Chang YF, Martin SS, Baldwin EP, Carman GM (2007) Phosphorylation of human CTP synthetase 1 by protein kinase C: identification of $\operatorname{Ser}(462)$ and $\operatorname{Thr}(455)$ as major sites of phosphorylation. J Biol Chem 282(24):17613-17622

Chang CC, Lin WC, Pai LM, Lee HS, Wu SC, Ding ST, Liu JL, Sung LY (2015) Cytoophidium assembly reflects upregulation of IMPDH activity. J Cell Sci 128(19):3550-3555

Chang CC, Keppeke GD, Sung LY, Liu JL (2018) Interfilament interaction between IMPDH and CTPS cytoophidia. FEBS J 285(20): 3753-3768

Chaudhry FA, Reimer RJ, Edwards RH (2002) The glutamine commute: take the N line and transfer to the A. J Cell Biol 157(3):349-355

Chen Y, Brandizzi F (2013) IRE1: ER stress sensor and cell fate executor. Trends Cell Biol 23(11):547-555

Chen L, Pankiewicz KW (2007) Recent development of IMP dehydrogenase inhibitors for the treatment of cancer. Curr Opin Drug Discov Devel 10(4):403-412

Chen Y, Zhu J, Lum PY, Yang X, Pinto S, MacNeil DJ, Zhang C, Lamb J, Edwards S, Sieberts SK, Leonardson A, Castellini LW, Wang S, Champy MF, Zhang B, Emilsson V, Doss S, Ghazalpour A, Horvath S, Drake TA, Lusis AJ, Schadt EE (2008a) Variations in DNA elucidate molecular networks that cause disease. Nature 452(7186):429-435

Chen Z, Yang H, Pavletich NP (2008b) Mechanism of homologous recombination from the RecA-ssDNA/dsDNA structures. Nature 453(7194):489-484

Chen K, Zhang J, Tastan OY, Deussen ZA, Siswick MY, Liu JL (2011) Glutamine analogs promote cytoophidium assembly in human and Drosophila cells. J Genet Genomics 38(9):391-402

Chiti F, Dobson CM (2006) Protein misfolding, functional amyloid, and human disease. Annu Rev Biochem 75:333-366

Cho YS, Challa S, Moquin D, Genga R, Ray TD, Guildford M, Chan FK (2009) Phosphorylation-driven assembly of the RIP1-RIP3 complex regulates programmed necrosis and virus-induced inflammation. Cell 137(6):1112-1123

Choi MG, Park TS, Carman GM (2003) Phosphorylation of Saccharomyces cerevisiae CTP synthetase at Ser424 by protein kinases $\mathrm{A}$ and $\mathrm{C}$ regulates phosphatidylcholine synthesis by the CDPcholine pathway. J Biol Chem 278(26):23610-23616

Cimini A, Gentile R, Angelucci F, Benedetti E, Pitari G, Giordano A, Ippoliti R (2013) Neuroprotective effects of PrxI over-expression in an in vitro human Alzheimer's disease model. J Cell Biochem 114(3):708-715

Cox JS, Shamu CE, Walter P (1993) Transcriptional induction of genes encoding endoplasmic reticulum resident proteins requires a transmembrane protein kinase. Cell 73(6):1197-1206

Credle JJ, Finer-Moore JS, Papa FR, Stroud RM, Walter P (2005) On the mechanism of sensing unfolded protein in the endoplasmic reticulum. Proc Natl Acad Sci U S A 102(52):18773-18784

Cutler SR, Somerville CR (2005) Imaging plant cell death: GFP-Nit1 aggregation marks an early step of wound and herbicide induced cell death. BMC Plant Biol 5:4

Daniels LE, Wood KM, Scott DJ, Halford SE (2003) Subunit assembly for DNA cleavage by restriction endonuclease SgrAI. J Mol Biol 327(3):579-591
DeBerardinis RJ, Cheng T (2010) Q's next: the diverse functions of glutamine in metabolism, cell biology and cancer. Oncogene 29(3): 313-324

Dechant R, Binda M, Lee SS, Pelet S, Winderickx J, Peter M (2010) Cytosolic $\mathrm{pH}$ is a second messenger for glucose and regulates the PKA pathway through V-ATPase. EMBO J 29(15):2515-2526

DeLaBarre B, Gross S, Fang C, Gao Y, Jha A, Jiang F, Song JJ, Wei W, Hurov JB (2011) Full-length human glutaminase in complex with an allosteric inhibitor. Biochemistry 50(50):10764-10770

Deng Y, Gam J, French JB, Zhao H, An S, Benkovic SJ (2012) Mapping protein-protein proximity in the purinosome. J Biol Chem 287(43): 36201-36207

Dent KC, Weber BW, Benedik MJ, Sewell BT (2009) The cyanide hydratase from Neurospora crassa forms a helix which has a dimeric repeat. Appl Microbiol Biotechnol 82(2):271-278

Dever TE, Yang W, Astrom S, Bystrom AS, Hinnebusch AG (1995) Modulation of tRNA(iMet), eIF-2, and eIF-2B expression shows that GCN4 translation is inversely coupled to the level of eIF2.GTP.Met-tRNA(iMet) ternary complexes. Mol Cell Biol 15(11): 6351-6363

Diebolder CA, Halff EF, Koster AJ, Huizinga EG, Koning RI (2015) Cryoelectron tomography of the NAIP5/NLRC4 inflammasome: implications for NLR activation. Structure 23(12):2349-2357

Dieter H, Koberstein R, Sund H (1981) Studies of glutamate dehydrogenase. The interaction of ADP, GTP, and NADPH in complexes with glutamate dehydrogenase. Eur J Biochem 115(1):217-226

Duderstadt KE, Chuang K, Berger JM (2011) DNA stretching by bacterial initiators promotes replication origin opening. Nature 478(7368):209-213

Dunten PW, Little EJ, Gregory MT, Manohar VM, Dalton M, Hough D, Bitinaite J, Horton NC (2008) The structure of SgrAI bound to DNA; recognition of an 8 base pair target. Nucleic Acids Res 36: 5405-5416

Eisenberg H, Reisler E (1971) Angular dependence of scattered light, rotary frictional coefficients, and distribution of sizes of associated oligomers in solutions of bovine liver glutamate dehydrogenase. Biopolymers 10(12):2363-2376

Eisenberg D, Gill HS, Pfluegl GM, Rotstein SH (2000) Structurefunction relationships of glutamine synthetases. Biochim Biophys Acta 1477(1-2):122-145

Endrizzi JA, Kim H, Anderson PM, Baldwin EP (2005) Mechanisms of product feedback regulation and drug resistance in cytidine triphosphate synthetases from the structure of a CTP-inhibited complex. Biochemistry 44(41):13491-13499

Erdmann N, Zhao J, Lopez AL, Herek S, Curthoys N, Hexum TD, Tsukamoto T, Ferraris D, Zheng J (2007) Glutamate production by HIV-1 infected human macrophage is blocked by the inhibition of glutaminase. J Neurochem 102(2):539-549

Erdmann N, Tian C, Huang Y, Zhao J, Herek S, Curthoys N, Zheng J (2009) In vitro glutaminase regulation and mechanisms of glutamate generation in HIV-1-infected macrophage. J Neurochem 109(2): 551-561

Erzberger JP, Mott ML, Berger JM (2006) Structural basis for ATPdependent DnaA assembly and replication-origin remodeling. Nat Struct Mol Biol 13(8):676-683

Extance J, Crennell SJ, Eley K, Cripps R, Hough DW, Danson MJ (2013) Structure of a bifunctional alcohol dehydrogenase involved in bioethanol generation in Geobacillus thermoglucosidasius. Acta Crystallogr D Biol Crystallogr 69(Pt 10):2104-2115

Fahien LA, Macdonald MJ (2011) The complex mechanism of glutamate dehydrogenase in insulin secretion. Diabetes 60(10):2450-2454

Fahien LA, MacDonald MJ, Teller JK, Fibich B, Fahien CM (1989) Kinetic advantages of hetero-enzyme complexes with glutamate dehydrogenase and the alpha-ketoglutarate dehydrogenase complex. J Biol Chem 264(21):12303-12312 
Fahien LA, Teller JK, Macdonald MJ, Fahien CM (1990) Regulation of glutamate dehydrogenase by $\mathrm{Mg} 2+$ and magnification of leucine activation by $\mathrm{Mg} 2+$. Mol Pharmacol 37(6):943-949

Fernandes BCM, Mateo C, Kiziak C, Chmura A, Wacker J, van Rantwijk F, Stolz A, Sheldon RA (2006) Nitrile hydratase activity of a recombinant nitrilase. Adv Synth Catal 348:2597-2603

Ferrao R, Wu H (2012) Helical assembly in the death domain (DD) superfamily. Curr Opin Struct Biol 22(2):241-247

Ferreira AP, Cassago A, Goncalves Kde A, Dias MM, Adamoski D, Ascencao CF, Honorato RV, de Oliveira JF, Ferreira IM, Fornezari C, Bettini J, Oliveira PS, Paes Leme AF, Portugal RV, Ambrosio AL, Dias SM (2013) Active glutaminase C self-assembles into a supratetrameric oligomer that can be disrupted by an allosteric inhibitor. J Biol Chem 288(39):28009-28020

Foe LG, Trujillo JL (1980) Quaternary structure of pig liver phosphofructokinase. J Biol Chem 255(21):10537-10541

Frey TG, Eisenberg D, Eiserling FA (1975) Glutamine synthetase forms three- and seven-stranded helical cables. Proc Natl Acad Sci U S A 72(9):3402-3406

Frieden C (1959a) Glutamic dehydrogenase. I. The effect of coenzyme on the sedimentation velocity and kinetic behavior. J Biol Chem 234(4):809-814

Frieden C (1959b) Glutamic dehydrogenase. II. The effect of various nucleotides on the association-dissociation and kinetic properties. J Biol Chem 234(4):815-820

Frieden C (1965) Glutamate Dehydrogenase. Vi. Survey of Purine Nucleotide and Other Effects on the Enzyme from Various Sources. J Biol Chem 240:2028-2035

Garcia-Seisdedos H, Empereur-Mot C, Elad N, Levy ED (2017) Proteins evolve on the edge of supramolecular self-assembly. Nature 548(7666):244-247

Gardner BM, Walter P (2011) Unfolded proteins are Ire1-activating ligands that directly induce the unfolded protein response. Science 333(6051):1891-1894

Ghosh R, Wang L, Wang ES, Perera BG, Igbaria A, Morita S, Prado K, Thamsen M, Caswell D, Macias H, Weiberth KF, Gliedt MJ, Alavi MV, Hari SB, Mitra AK, Bhhatarai B, Schurer SC, Snapp EL, Gould DB, German MS, Backes BJ, Maly DJ, Oakes SA, Papa FR (2014) Allosteric inhibition of the IRE1alpha RNase preserves cell viability and function during endoplasmic reticulum stress. Cell 158(3):534 548

Gilliland WD, Vietti DL, Schweppe NM, Guo F, Johnson TJ, Hawley RS (2009) Hypoxia transiently sequesters $\mathrm{mps} 1$ and polo to collagenase-sensitive filaments in Drosophila prometaphase oocytes. PLoS One 4(10):e7544

Glover CV (1986) A filamentous form of Drosophila casein kinase II. J Biol Chem 261(30):14349-14354

Gong Q, Long Z, Zhong FL, Teo DET, Jin Y, Yin Z, Boo ZZ, Zhang Y, Zhang J, Yang R, Bhushan S, Reversade B, Li Z, Wu B (2018) Structural basis of RIP2 activation and signaling. Nat Commun 9(1):4993

Goodsell DS, Olson AJ (2000) Structural symmetry and protein function. Annu Rev Biophys Biomol Struct 29:105-153

Goto M, Omi R, Nakagawa N, Miyahara I, Hirotsu K (2004) Crystal structures of CTP synthetase reveal ATP, UTP, and glutamine binding sites. Structure 12(8):1413-1423

Gourlay LJ, Bhella D, Kelly SM, Price NC, Lindsay JG (2003) Structurefunction analysis of recombinant substrate protein $22 \mathrm{kDa}(\mathrm{SP}-22)$. A mitochondrial 2-CYS peroxiredoxin organized as a decameric toroid. J Biol Chem 278(35):32631-32637

Grabowska A, Nowicki M, Kwinta J (2011) Glutamate dehydrogenase of the germinating triticale seeds: gene expression, activity distribution and kinetic characteristics. Acta Physiol Plant 33(5):1981-1990

Griffin MDW, Gerrard JA (2012) The relationship between oligomeric state and protein function. In: Matthews JM (ed) Protein
Dimerization and Oligomerization in Biology. Landes Bioscience and Springer Science+Business Media, Austin, pp 74-90

Gunning BE (1965) The fine structure of chloroplast stroma following aldehyde osmium-tetroxide fixation. J Cell Biol 24:79-93

Gunter JH, Thomas EC, Lengefeld N, Kruger SJ, Worton L, Gardiner EM, Jones A, Barnett NL, Whitehead JP (2008) Characterisation of inosine monophosphate dehydrogenase expression during retinal development: differences between variants and isoforms. Int J Biochem Cell Biol 40(9):1716-1728

Guri Y, Colombi M, Dazert E, Hindupur SK, Roszik J, Moes S, Jenoe P, Heim MH, Riezman I, Riezman H, Hall MN (2017) mTORC2 promotes tumorigenesis via lipid synthesis. Cancer Cell 32(6):807-823 e812

Gylfe E (1976) Comparison of the effects of leucines, non-metabolizable leucine analogues and other insulin secretagogues on the activity of glutamate dehydrogenase. Acta Diabetol Lat 13(1-2):20-24

Ha J, Daniel S, Broyles SS, Kim KH (1994) Critical phosphorylation sites for acetyl-CoA carboxylase activity. J Biol Chem 269(35):2216222168

Hamberger A, Chiang GH, Sandoval E, Cotman CW (1979) Glutamate as a CNS transmitter. II. Regulation of synthesis in the releasable pool. Brain Res 168(3):531-541

Han GS, Sreenivas A, Choi MG, Chang YF, Martin SS, Baldwin EP, Carman GM (2005) Expression of Human CTP synthetase in Saccharomyces cerevisiae reveals phosphorylation by protein kinase A. J Biol Chem 280(46):38328-38336

Han D, Lerner AG, Vande Walle L, Upton JP, Xu W, Hagen A, Backes BJ, Oakes SA, Papa FR (2009) IRE1alpha kinase activation modes control alternate endoribonuclease outputs to determine divergent cell fates. Cell 138(3):562-575

Hanahan D, Weinberg RA (2011) Hallmarks of cancer: the next generation. Cell 144(5):646-674

Harper DB (1977a) Fungal degradation of aromatic nitriles. Enzymology of C-N cleavage by Fusarium solani. Biochem J 167(3):685-692

Harper DB (1977b) Microbial metabolism of aromatic nitriles. Enzymology of C-N cleavage by Nocardia sp. (Rhodochrous group) N.C.I.B. 11216. Biochem J 165(2):309-319

Harwood HJ Jr (2004) Acetyl-CoA carboxylase inhibition for the treatment of metabolic syndrome. Curr Opin Investig Drugs 5(3):283289

He S, Wang L, Miao L, Wang T, Du F, Zhao L, Wang X (2009a) Receptor interacting protein kinase-3 determines cellular necrotic response to TNF-alpha. Cell 137(6):1100-1111

He YX, Gui L, Liu YZ, Du Y, Zhou Y, Li P, Zhou CZ (2009b) Crystal structure of Saccharomyces cerevisiae glutamine synthetase Gln1 suggests a nanotube-like supramolecular assembly. Proteins 76(1): $249-254$

Hedstrom L (2009) IMP dehydrogenase: structure, mechanism, and inhibition. Chem Rev 109(7):2903-2928

Hindenburg AA, Taub RN, Grant S, Chang G, Baker MA (1985) Effects of pyrimidine antagonists on sialic acid regeneration in HL-60 cells. Cancer Res 45(7):3048-3052

Hingorani-Varma K, Bitinaite J (2003) Kinetic analysis of the coordinated interaction of SgrAI restriction endonuclease with different DNA targets. J Biol Chem 278(41):40392-40399

Hofer A, Steverding D, Chabes A, Brun R, Thelander L (2001) Trypanosoma brucei CTP synthetase: a target for the treatment of African sleeping sickness. Proc Natl Acad Sci U S A 98(11):64126416

Hoffman EM, Schechter R, Miller KE (2010) Fixative composition alters distributions of immunoreactivity for glutaminase and two markers of nociceptive neurons, Nav1.8 and TRPV1, in the rat dorsal root ganglion. J Histochem Cytochem 58(4):329-344

Hollien J, Weissman JS (2006) Decay of endoplasmic reticulum-localized mRNAs during the unfolded protein response. Science 313(5783): 104-107 
Hollien J, Lin JH, Li H, Stevens N, Walter P, Weissman JS (2009) Regulated Ire1-dependent decay of messenger RNAs in mammalian cells. J Cell Biol 186(3):323-331

Huang CY, Frieden C (1972) The mechanism of ligand-induced structural changes in glutamate dehydrogenase. Studies of the rate of depolymerization and isomerization effected by coenzymes and guanine nucleotides. J Biol Chem 247(11):3638-3646

Hubner GM, Larsen JN, Guerra B, Niefind K, Vrecl M, Issinger OG (2014) Evidence for aggregation of protein kinase CK2 in the cell: a novel strategy for studying CK2 holoenzyme interaction by BRET(2). Mol Cell Biochem 397(1-2):285-293

Hudson RC, Daniel RM (1993) L-glutamate dehydrogenases: distribution, properties and mechanism. Comp Biochem Physiol B 106(4): 767-792

Hunkeler M, Hagmann A, Stuttfeld E, Chami M, Guri Y, Stahlberg H, Maier T (2018) Structural basis for regulation of human acetyl-CoA carboxylase. Nature 558(7710):470-474

Ibstedt S, Sideri TC, Grant CM, Tamas MJ (2014) Global analysis of protein aggregation in yeast during physiological conditions and arsenite stress. Biol Open 3(10):913-923

Imagawa Y, Hosoda A, Sasaka S, Tsuru A, Kohno K (2008) RNase domains determine the functional difference between IRE1alpha and IRE1beta. FEBS Lett 582(5):656-660

Ingerson-Mahar M, Briegel A, Werner JN, Jensen GJ, Gitai Z (2010) The metabolic enzyme CTP synthase forms cytoskeletal filaments. Nat Cell Biol 12(8):739-746

Ishiwata-Kimata Y, Yamamoto YH, Takizawa K, Kohno K, Kimata Y (2013) F-actin and a type-II myosin are required for efficient clustering of the ER stress sensor Ire1. Cell Struct Funct 38(2):135-143

Isom DG, Sridharan V, Baker R, Clement ST, Smalley DM, Dohlman HG (2013) Protons as second messenger regulators of $G$ protein signaling. Mol Cell 51(4):531-538

Iwawaki T, Hosoda A, Okuda T, Kamigori Y, Nomura-Furuwatari C, Kimata Y, Tsuru A, Kohno K (2001) Translational control by the ER transmembrane kinase/ribonuclease IRE1 under ER stress. Nat Cell Biol 3(2):158-164

Jacobson T, Navarrete C, Sharma SK, Sideri TC, Ibstedt S, Priya S, Grant CM, Christen P, Goloubinoff P, Tamas MJ (2012) Arsenite interferes with protein folding and triggers formation of protein aggregates in yeast. J Cell Sci 125(Pt 21):5073-5083

Jain S, Wheeler JR, Walters RW, Agrawal A, Barsic A, Parker R (2016) ATPase-modulated stress granules contain a diverse proteome and substructure. Cell 164(3):487-498

Jang S, Nelson JC, Bend EG, Rodriguez-Laureano L, Tueros FG, Cartagenova L, Underwood K, Jorgensen EM, Colon-Ramos DA (2016) Glycolytic enzymes localize to synapses under energy stress to support synaptic function. Neuron 90(2):278-291

Jang SR, Xuan Z, Lagoy RC, Jawerth LM, Gonzalez I, Singh M, Prashad S, Kim HS, Patel AR, Albrecht DR, Hyman AA, Colon-Ramos DA (2019) The glycolytic protein phosphofructokinase dynamically relocalizes into subcellular compartments with liquid-like properties in vivo. BioRxiy

Ji Y, Gu J, Makhov AM, Griffith JD, Mitchell BS (2006) Regulation of the interaction of inosine monophosphate dehydrogenase with mycophenolic Acid by GTP. J Biol Chem 281(1):206-212

Jin M, Fuller GG, Han T, Yao Y, Alessi AF, Freeberg MA, Roach NP, Moresco JJ, Karnovsky A, Baba M, Yates JR 3rd, Gitler AD, Inoki K, Klionsky DJ, Kim JK (2017) Glycolytic enzymes coalesce in g bodies under hypoxic stress. Cell Rep 20(4):895-908

Josephs R, Borisy G (1972) Self-assembly of glutamic dehydrogenase into ordered superstructures: multichain tubes formed by association of single molecules. J Mol Biol 65(1):127-155

Kaguni JM (2006) DnaA: controlling the initiation of bacterial DNA replication and more. Annu Rev Microbiol 60:351-375
Kang GJ, Cooney DA, Moyer JD, Kelley JA, Kim HY, Marquez VE, Johns DG (1989) Cyclopentenylcytosine triphosphate. Formation and inhibition of CTP synthetase. J Biol Chem 264(2):713-718

Karagoz GE, Acosta-Alvear D, Nguyen HT, Lee CP, Chu F, Walter P (2017) An unfolded protein-induced conformational switch activates mammalian IRE1. Elife 6

Katayama T, Ozaki S, Keyamura K, Fujimitsu K (2010) Regulation of the replication cycle: conserved and diverse regulatory systems for DnaA and oriC. Nat Rev Microbiol 8(3):163-170

Kawata T, Masuda K, Yoshino K (1975) Presence of fine spirals (spirosomes) in Lactobacillus fermenti and Lactobacillus casei. Jpn J Microbiol 19(3):225-227

Kemp RG (1971) Rabbit liver phosphofructokinase. Comparison of some properties with those of muscle phosphofructokinase. J Biol Chem 246(1):245-252

Keppeke GD, Calise SJ, Chan EK, Andrade LE (2015) Assembly of IMPDH2-based, CTPS-based, and mixed rod/ring structures is dependent on cell type and conditions of induction. J Genet Genomics 42(6):287-299

Keppeke GD, Chang CC, Peng M, Chen LY, Lin WC, Pai LM, Andrade LEC, Sung LY, Liu JL (2018) IMP/GTP balance modulates cytoophidium assembly and IMPDH activity. Cell Div 13:5

Kessler D, Leibrecht I, Knappe J (1991) Pyruvate-formate-lyasedeactivase and acetyl-CoA reductase activities of Escherichia coli reside on a polymeric protein particle encoded by adhE. FEBS Lett 281(1-2):59-63

Kessler D, Herth W, Knappe J (1992) Ultrastructure and pyruvate formate-lyase radical quenching property of the multienzymic AdhE protein of Escherichia coli. J Biol Chem 267(25):1807318079

Kim YW, Kang KS, Kim SY, Kim IS (2000) Formation of fibrillar multimers of oat beta-glucosidase isoenzymes is mediated by the As-Glu1 monomer. J Mol Biol 303(5):831-842

Kim SY, Kim YW, Hegerl R, Cyrklaff M, Kim IS (2005) Novel type of enzyme multimerization enhances substrate affinity of oat beta-glucosidase. J Struct Biol 150(1):1-10

Kim CW, Moon YA, Park SW, Cheng D, Kwon HJ, Horton JD (2010) Induced polymerization of mammalian acetyl-CoA carboxylase by MIG12 provides a tertiary level of regulation of fatty acid synthesis. Proc Natl Acad Sci U S A 107(21):9626-9631

Kimmig P, Diaz M, Zheng J, Williams CC, Lang A, Aragon T, Li H, Walter $P$ (2012) The unfolded protein response in fission yeast modulates stability of select mRNAs to maintain protein homeostasis. Elife 1:e00048

Kleinschmidt AK, Moss J, Lane DM (1969) Acetyl coenzyme A carboxylase: filamentous nature of the animal enzymes. Science 166(3910):1276-1278

Kloos M, Bruser A, Kirchberger J, Schoneberg T, Strater N (2014) Crystallization and preliminary crystallographic analysis of human muscle phosphofructokinase, the main regulator of glycolysis. Acta Crystallogr F Struct Biol Commun 70(Pt 5):578-582

Kloos M, Bruser A, Kirchberger J, Schoneberg T, Strater N (2015) Crystal structure of human platelet phosphofructokinase-1 locked in an activated conformation. Biochem J 469(3):421-432

Kobayashi I (1996) DNA modification and restriction: selfish behavior of an epigenetic system. In: Russo VEA, Martienssen RA, Riggs AD (eds) Epigenetic Mechanisms of Gene Regulation. Cold Spring Harbor Laboratory Press, Cold Spring Harbor, pp 155-172

Kohnhorst CL, Kyoung M, Jeon M, Schmitt DL, Kennedy EL, Ramirez J, Bracey SM, Luu BT, Russell SJ, An S (2017) Identification of a multienzyme complex for glucose metabolism in living cells. J Biol Chem 292(22):9191-9203

Korennykh AV, Egea PF, Korostelev AA, Finer-Moore J, Zhang C, Shokat KM, Stroud RM, Walter P (2009) The unfolded protein response signals through high-order assembly of Ire1. Nature 457(7230):687-693 
Kozhevnikova EN, van der Knaap JA, Pindyurin AV, Ozgur Z, van Ijcken WF, Moshkin YM, Verrijzer CP (2012) Metabolic enzyme IMPDH is also a transcription factor regulated by cellular state. Mol Cell 47(1):133-139

Kreis T, Vale R (1999) Guidebook to the cytoskeletal and motor proteins. Oxford University Press, Oxford

Kumsta C, Jakob U (2009) Redox-regulated chaperones. Biochemistry 48(22):4666-4676

Kursula P, Flodin S, Ehn M, Hammarstrom M, Schuler H, Nordlund P, Stenmark P (2006) Structure of the synthetase domain of human CTP synthetase, a target for anticancer therapy. Acta Crystallogr Sect F Struct Biol Cryst Commun 62(Pt 7):613-617

Labesse G, Alexandre T, Vaupre L, Salard-Arnaud I, Him JL, Raynal B, Bron P, Munier-Lehmann H (2013) MgATP regulates allostery and fiber formation in IMPDHs. Structure 21(6):975-985

Laurenceau R, Krasteva PV, Diallo A, Ouarti S, Duchateau M, Malosse C, Chamot-Rooke J, Fronzes R (2015) Conserved Streptococcus pneumoniae spirosomes suggest a single type of transformation pilus in competence. PLoS Pathog 11(4):e1004835

Lauritsen I, Willemoes M, Jensen KF, Johansson E, Harris P (2011) Structure of the dimeric form of CTP synthase from Sulfolobus solfataricus. Acta Crystallogr Sect F Struct Biol Cryst Commun 67(Pt 2):201-208

Lee KP, Dey M, Neculai D, Cao C, Dever TE, Sicheri F (2008) Structure of the dual enzyme Ire1 reveals the basis for catalysis and regulation in nonconventional RNA splicing. Cell 132(1):89-100

Leonard AC, Grimwade JE (2010) Regulating DnaA complex assembly: it is time to fill the gaps. Curr Opin Microbiol 13(6):766-772

Li H, Korennykh AV, Behrman SL, Walter P (2010) Mammalian endoplasmic reticulum stress sensor IRE1 signals by dynamic clustering. Proc Natl Acad Sci U S A 107(37):16113-16118

Li M, Li C, Allen A, Stanley CA, Smith TJ (2011) The structure and allosteric regulation of glutamate dehydrogenase. Neurochem Int 59(4):445-455

Li J, McQuade T, Siemer AB, Napetschnig J, Moriwaki K, Hsiao YS, Damko E, Moquin D, Walz T, McDermott A, Chan FK, Wu H (2012a) The RIP1/RIP3 necrosome forms a functional amyloid signaling complex required for programmed necrosis. Cell 150(2):339350

Li M, Li C, Allen A, Stanley CA, Smith TJ (2012b) The structure and allosteric regulation of mammalian glutamate dehydrogenase. Arch Biochem Biophys 519(2):69-80

Li W, Okreglak V, Peschek J, Kimmig P, Zubradt M, Weissman JS, Walter P (2018) Engineering ER-stress dependent nonconventional mRNA splicing. Elife 7

Lightfoot DA, Baron AJ, Wootton JC (1988) Expression of the Escherichia coli glutamate dehydrogenase gene in the cyanobacterium Synechococcus PCC6301 causes ammonium tolerance. Plant Mol Biol 11(3):335-344

Lightfoot DA, Bernhardt K, Mungur R, Nolte S, Ameziane R, Colter A, Jones K, Iqbal MJ, Varsa E, Young B (2007) Improved drought tolerance of transgenic Zea mays plants that express the glutamate dehydrogenase gene (gdhA) of E. coli. Euphytica 156:103-116

Lin SC, Lo YC, Wu H (2010) Helical assembly in the MyD88-IRAK4IRAK2 complex in TLR/IL-1R signalling. Nature 465(7300):885890

Litchfield DW (2003) Protein kinase CK2: structure, regulation and role in cellular decisions of life and death. Biochem J 369(Pt 1):1-15

Little EJ, Dunten PW, Bitinaite J, Horton NC (2011) New clues in the allosteric activation of DNA cleavage by SgrAI: structures of SgrAI bound to cleaved primary-site DNA and uncleaved secondary-site DNA. Acta Crystallogr D Biol Crystallogr 67(Pt 1):67-74

Liu JL (2010) Intracellular compartmentation of CTP synthase in Drosophila. J Genet Genomics 37(5):281-296
Liu JL (2016) The cytoophidium and its kind: filamentation and compartmentation of metabolic enzymes. Annu Rev Cell Dev Biol 32:349 372

Lolli G, Ranchio A, Battistutta R (2014) Active form of the protein kinase CK2 alpha2beta2 holoenzyme is a strong complex with symmetric architecture. ACS Chem Biol 9(2):366-371

Lowe N, Rees JS, Roote J, Ryder E, Armean IM, Johnson G, Drummond E, Spriggs H, Drummond J, Magbanua JP, Naylor H, Sanson B, Bastock R, Huelsmann S, Trovisco V, Landgraf M, KnowlesBarley S, Armstrong JD, White-Cooper H, Hansen C, Phillips RG, Consortium UKDPTS, Lilley KS, Russell S, St Johnston D (2014) Analysis of the expression patterns, subcellular localisations and interaction partners of Drosophila proteins using a pigP protein trap library. Development 141(20):3994-4005

$\mathrm{Lu} \mathrm{A,} \mathrm{Wu} \mathrm{H} \mathrm{(2015)} \mathrm{Structural} \mathrm{mechanisms} \mathrm{of} \mathrm{inflammasome} \mathrm{assembly.}$ FEBS J 282(3):435-444

Lu A, Magupalli VG, Ruan J, Yin Q, Atianand MK, Vos MR, Schroder GF, Fitzgerald KA, Wu H, Egelman EH (2014a) Unified polymerization mechanism for the assembly of ASC-dependent inflammasomes. Cell 156(6):1193-1206

Lu Y, Liang FX, Wang X (2014b) A synthetic biology approach identifies the mammalian UPR RNA ligase RtcB. Mol Cell 55(5):758-770

Lu A, Li Y, Schmidt FI, Yin Q, Chen S, Fu TM, Tong AB, Ploegh HL, Mao Y, Wu H (2016) Molecular basis of caspase-1 polymerization and its inhibition by a new capping mechanism. Nat Struct Mol Biol 23(5):416-425

Lusetti SL, Cox MM (2002) The bacterial RecA protein and the recombinational DNA repair of stalled replication forks. Annu Rev Biochem 71:71-100

Lynch EM, Hicks DR, Shepherd M, Endrizzi JA, Maker A, Hansen JM, Barry RM, Gitai Z, Baldwin EP, Kollman JM (2017) Human CTP synthase filament structure reveals the active enzyme conformation. Nat Struct Mol Biol 24(6):507-514

Lyumkis D, Talley H, Stewart A, Shah S, Park CK, Tama F, Potter CS, Carragher B, Horton NC (2013) Allosteric regulation of DNA cleavage and sequence-specificity through run-on oligomerization. Structure 21(10): 1848-1858

Ma X, Shah S, Zhou M, Park CK, Wysocki VH, Horton NC (2013) Structural analysis of activated SgrAI-DNA oligomers using ion mobility mass spectrometry. Biochemistry 52(25):4373-4381

Magnard C, Bachelier R, Vincent A, Jaquinod M, Kieffer S, Lenoir GM, Venezia ND (2002) BRCA1 interacts with acetyl-CoA carboxylase through its tandem of BRCT domains. Oncogene 21(44):67296739

Maia LB, Moura JJ, Moura I (2015) Molybdenum and tungstendependent formate dehydrogenases. J Biol Inorg Chem 20(2):287309

Maitra PK (1970) A glucokinase from Saccharomyces cerevisiae. J Biol Chem 245(9):2423-2431

Mamrack MD (1989) Stimulation of enzymatic activity in filament preparations of casein kinase II by polylysine, melittin, and spermine. Mol Cell Biochem 85(2):147-157

Martin E, Palmic N, Sanquer S, Lenoir C, Hauck F, Mongellaz C, Fabrega S, Nitschke P, Esposti MD, Schwartzentruber J, Taylor N, Majewski J, Jabado N, Wynn RF, Picard C, Fischer A, Arkwright PD, Latour S (2014) CTP synthase 1 deficiency in humans reveals its central role in lymphocyte proliferation. Nature 510(7504):288 292

Mates JM, Segura JA, Campos-Sandoval JA, Lobo C, Alonso L, Alonso FJ, Marquez J (2009) Glutamine homeostasis and mitochondrial dynamics. Int J Biochem Cell Biol 41(10):2051-2061

Maurel M, Chevet E, Tavernier J, Gerlo S (2014) Getting RIDD of RNA: IRE1 in cell fate regulation. Trends Biochem Sci 39(5):245-254

Meggio F, Pinna LA (2003) One-thousand-and-one substrates of protein kinase CK2? FASEB J 17(3):349-368 
Meredith MJ, Lane MD (1978) Acetyl-CoA carboxylase. Evidence for polymeric filament to protomer transition in the intact avian liver cell. J Biol Chem 253(10):3381-3383

Miller RE, Shelton E, Stadtman ER (1974) Zinc-induced paracrystalline aggregation of glutamine synthetase. Arch Biochem Biophys 163(1):155-171

Mishiba K, Nagashima Y, Suzuki E, Hayashi N, Ogata Y, Shimada Y, Koizumi N (2013) Defects in IRE1 enhance cell death and fail to degrade mRNAs encoding secretory pathway proteins in the Arabidopsis unfolded protein response. Proc Natl Acad Sci U S A 110(14):5713-5718

Miura N, Shinohara M, Tatsukami Y, Sato Y, Morisaka H, Kuroda K, Ueda M (2013) Spatial reorganization of Saccharomyces cerevisiae enolase to alter carbon metabolism under hypoxia. Eukaryot Cell 12(8):1106-1119

Mompean M, Li W, Li J, Laage S, Siemer AB, Bozkurt G, Wu H, McDermott AE (2018) The Structure of the necrosome RIPK1RIPK3 core, a human hetero-amyloid signaling complex. Cell 173(5): 1244-1253 e1210

Moon JC, Hah YS, Kim WY, Jung BG, Jang HH, Lee JR, Kim SY, Lee YM, Jeon MG, Kim CW, Cho MJ, Lee SY (2005) Oxidative stressdependent structural and functional switching of a human 2-Cys peroxiredoxin isotype II that enhances HeLa cell resistance to H2O2-induced cell death. J Biol Chem 280(31):28775-28784

Moore K, Hollien J (2015) Ire1-mediated decay in mammalian cells relies on mRNA sequence, structure, and translational status. Mol Biol Cell 26(16):2873-2884

Moquin D, Chan FK (2010) The molecular regulation of programmed necrotic cell injury. Trends Biochem Sci 35(8):434 441

Moreno-Sanchez R, Marin-Hernandez A, Gallardo-Perez JC, Quezada H, Encalada R, Rodriguez-Enriquez S, Saavedra E (2012) Phosphofructokinase type 1 kinetics, isoform expression, and gene polymorphisms in cancer cells. J Cell Biochem 113(5):1692-1703

Mori K, Ma W, Gething MJ, Sambrook J (1993) A transmembrane protein with a cdc2+/CDC28-related kinase activity is required for signaling from the ER to the nucleus. Cell 74(4):743-756

Moss J, Lane MD (1972) Acetyl coenzyme A carboxylase. IV. Biotinyl prosthetic group-independent malonyl coenzyme A decarboxylation and carbosyl transfer: generalization to other biotin enzymes. J Biol Chem 247(16):4952-4959

Mungur R, Glass AD, Goodenow DB, Lightfoot DA (2005) Metabolite fingerprinting in transgenic Nicotiana tabacum altered by the Escherichia coli glutamate dehydrogenase gene. J Biomed Biotechnol 2005(2):198-214

Nagai M, Natsumeda Y, Konno Y, Hoffman R, Irino S, Weber G (1991) Selective up-regulation of type II inosine 5'-monophosphate dehydrogenase messenger RNA expression in human leukemias. Cancer Res 51(15):3886-3890

Nagasawa T, Wieser M, Nakamura T, Iwahara H, Yoshida T, Gekko K (2000) Nitrilase of Rhodococcus rhodochrous J1. Conversion into the active form by subunit association. Eur J Biochem 267(1):138 144

Naidoo N, Giang W, Galante RJ, Pack AI (2005) Sleep deprivation induces the unfolded protein response in mouse cerebral cortex. J Neurochem 92(5):1150-1157

Nair V, Shu Q (2007) Inosine monophosphate dehydrogenase as a probe in antiviral drug discovery. Antivir Chem Chemother 18(5):245258

Nair V, Ma X, Shu Q, Zhang F, Uchil V, Cherukupalli GR (2007) IMPDH as a biological probe for RNA antiviral drug discovery: synthesis, enzymology, molecular docking, and antiviral activity of new ribonucleosides with surrogate bases. Nucleosides Nucleotides Nucleic Acids 26(6-7):651-654

Nakamura D, Tsuru A, Ikegami K, Imagawa Y, Fujimoto N, Kohno K (2011) Mammalian ER stress sensor IRE1beta specifically down- regulates the synthesis of secretory pathway proteins. FEBS Lett 585(1):133-138

Narayanaswamy R, Levy M, Tsechansky M, Stovall GM, O’Connell JD, Mirrielees J, Ellington AD, Marcotte EM (2009) Widespread reorganization of metabolic enzymes into reversible assemblies upon nutrient starvation. Proc Natl Acad Sci U S A 106(25):10147-10152

Niefind K, Guerra B, Ermakowa I, Issinger OG (2001) Crystal structure of human protein kinase CK2: insights into basic properties of the CK2 holoenzyme. EMBO J 20(19):5320-5331

Nisius A (1988) The stromacentre inAvena plastids: an aggregation ofbeta-glucosidase responsible for the activation of oat-leaf saponins. Planta 173(4):474-481

Nisius A, Ruppel HG (1987) Immunocytological and chemical studies on the stromacentre-forming protein from Avena plastids. Planta 171(4):443-452

Niwa M, Patil CK, DeRisi J, Walter P (2005) Genome-scale approaches for discovering novel nonconventional splicing substrates of the Ire1 nuclease. Genome Biol 6(1):R3

Noichri Y, Palais G, Ruby V, D'Autreaux B, Delaunay-Moisan A, Nystrom T, Molin M, Toledano MB (2015) In vivo parameters influencing 2-Cys Prx oligomerization: the role of enzyme sulfinylation. Redox Biol 6:326-333

Noree C, Sato BK, Broyer RM, Wilhelm JE (2010) Identification of novel filament-forming proteins in Saccharomyces cerevisiae and Drosophila melanogaster. J Cell Biol 190(4):541-551

Noree C, Monfort E, Shiau AK, Wilhelm JE (2014) Common regulatory control of CTP synthase enzyme activity and filament formation. Mol Biol Cell 25(15):2282-2290

Noree C, Begovich K, Samilo D, Broyer R, Monfort E, Wilhelm JE (2019a) A quantitative screen for metabolic enzyme structures reveals patterns of assembly across the yeast metabolic network. Mol Biol Cell 30(21):2721-2736

Noree C, Sirinonthanawech N, Wilhelm JE (2019b) Saccharomyces cerevisiae ASN1 and ASN2 are asparagine synthetase paralogs that have diverged in their ability to polymerize in response to nutrient stress. Sci Rep 9(1):278

Nuske E, Marini G, Richter D, Leng W, Bogdanova A, Franszmann T, Pigino G, Alberti S (2018) Filament formation by the translation factor eIF2B regulates protein synthesis in starved cells. bioRxiv

O'Connell JD, Zhao A, Ellington AD, Marcotte EM (2012) Dynamic reorganization of metabolic enzymes into intracellular bodies. Annu Rev Cell Dev Biol 28:89-111

O'Connell JD, Tsechansky M, Royall A, Boutz DR, Ellington AD, Marcotte EM (2014) A proteomic survey of widespread protein aggregation in yeast. Mol BioSyst 10(4):851-861

Olsen BR, Svenneby G, Kvamme E, Tveit B, Eskeland T (1970) Formation and ultrastructure of enzymically active polymers of pig renal glutaminase. J Mol Biol 52(2):239-245

Olson JA, Anfinsen CB (1952) The crystallization and characterization of L-glutamic acid dehydrogenase. J Biol Chem 197(1):67-79

Oosawa F, Asakura S (1975) Thermodynamics of the polymerization of protein. Academic Press Inc, London

Orij R, Postmus J, Ter Beek A, Brul S, Smits GJ (2009) In vivo measurement of cytosolic and mitochondrial $\mathrm{pH}$ using a $\mathrm{pH}$-sensitive GFP derivative in Saccharomyces cerevisiae reveals a relation between intracellular $\mathrm{pH}$ and growth. Microbiology 155(Pt 1):268-278

Orij R, Brul S, Smits GJ (2011) Intracellular pH is a tightly controlled signal in yeast. Biochim Biophys Acta 1810(10):933-944

Orij R, Urbanus ML, Vizeacoumar FJ, Giaever G, Boone C, Nislow C, Brul S, Smits GJ (2012) Genome-wide analysis of intracellular $\mathrm{pH}$ reveals quantitative control of cell division rate by $\mathrm{pH}(\mathrm{c})$ in Saccharomyces cerevisiae. Genome Biol 13(9):R80

Pace HC, Brenner C (2001) The nitrilase superfamily: classification, structure and function. Genome Biol 2(1):REVIEWS0001 
Pandey R, Heeger S, Lehner CF (2007) Rapid effects of acute anoxia on spindle kinetochore interactions activate the mitotic spindle checkpoint. J Cell Sci 120(Pt 16):2807-2818

Park CK, Stiteler AP, Shah S, Ghare MI, Bitinaite J, Horton NC (2010) Activation of DNA cleavage by oligomerization of DNA-bound SgrAI. Biochemistry 49(41):8818-8830

Park S, Hwang IW, Makishima Y, Perales-Clemente E, Kato T, Niederlander NJ, Park EY, Terzic A (2013) Spot14/Mig12 heterocomplex sequesters polymerization and restrains catalytic function of human acetyl-CoA carboxylase 2. J Mol Recognit 26(12):679-688

Park CK, Sanchez JL, Barahona C, Basantes LE, Sanchez J, Hernandez C, Horton NC (2018a) The run-on oligomer filament enzyme mechanism of SgrAI: Part 1. Assembly kinetics of the run-on oligomer filament. J Biol Chem 293(38):14585-14598

Park CK, Sanchez JL, Barahona C, Basantes LE, Sanchez J, Hernandez C, Horton NC (2018b) The run-on oligomer filament enzyme mechanism of SgrAI: Part 2. Kinetic modeling of the full DNA cleavage pathway. J Biol Chem 293(38):14599-14615

Peitsaro N, Polianskyte Z, Tuimala J, Porn-Ares I, Liobikas J, Speer O, Lindholm D, Thompson J, Eriksson O (2008) Evolution of a family of metazoan active-site-serine enzymes from penicillin-binding proteins: a novel facet of the bacterial legacy. BMC Evol Biol 8:26

Peters LZ, Hazan R, Breker M, Schuldiner M, Ben-Aroya S (2013) Formation and dissociation of proteasome storage granules are regulated by cytosolic pH. J Cell Biol 201(5):663-671

Petrovska I, Nuske E, Munder MC, Kulasegaran G, Malinovska L, Kroschwald S, Richter D, Fahmy K, Gibson K, Verbavatz JM, Alberti S (2014) Filament formation by metabolic enzymes is a specific adaptation to an advanced state of cellular starvation. Elife 3:e02409

Phalen TJ, Weirather K, Deming PB, Anathy V, Howe AK, van der Vliet A, Jonsson TJ, Poole LB, Heintz NH (2006) Oxidation state governs structural transitions in peroxiredoxin II that correlate with cell cycle arrest and recovery. J Cell Biol 175(5):779-789

Pingoud A, Wilson GG, Wende W (2014) Type II restriction endonucleases-a historical perspective and more. Nucleic Acids Res 42(12):7489-7527

Poehlein A, Schmidt S, Kaster AK, Goenrich M, Vollmers J, Thurmer A, Bertsch J, Schuchmann K, Voigt B, Hecker M, Daniel R, Thauer RK, Gottschalk G, Muller V (2012) An ancient pathway combining carbon dioxide fixation with the generation and utilization of a sodium ion gradient for ATP synthesis. PLoS One 7(3):e33439

Polianskyte Z, Peitsaro N, Dapkunas A, Liobikas J, Soliymani R, Lalowski M, Speer O, Seitsonen J, Butcher S, Cereghetti GM, Linder MD, Merckel M, Thompson J, Eriksson O (2009) LACTB is a filament-forming protein localized in mitochondria. Proc Natl Acad Sci U S A 106(45):18960-18965

Politi PM, Xie F, Dahut W, Ford H Jr, Kelley JA, Bastian A, Setser A, Allegra CJ, Chen AP, Hamilton JM et al (1995) Phase I clinical trial of continuous infusion cyclopentenyl cytosine. Cancer Chemother Pharmacol 36(6):513-523

Pollegioni L, Piubelli L, Sacchi S, Pilone MS, Molla G (2007) Physiological functions of D-amino acid oxidases: from yeast to humans. Cell Mol Life Sci 64(11):1373-1394

Polley S, Lyumkis D, Horton NC (2019) Mechanism of filamentationinduced allosteric activation of the SgrAI endonuclease. Structure 27(10):1497-1507

Poole A, Poore T, Bandhakavi S, McCann RO, Hanna DE, Glover CV (2005) A global view of CK2 function and regulation. Mol Cell Biochem 274(1-2):163-170

Poothong J, Tirasophon W, Kaufman RJ (2017) Functional analysis of the mammalian RNA ligase for IRE1 in the unfolded protein response. Biosci Rep 37(2)

Prouteau M, Loewith R (2018) Regulation of cellular metabolism through phase separation of enzymes. Biomolecules 8(4):160-173
Prouteau M, Desfosses A, Sieben C, Bourgoint C, Lydia Mozaffari N, Demurtas D, Mitra AK, Guichard P, Manley S, Loewith R (2017) TORC1 organized in inhibited domains (TOROIDs) regulate TORC1 activity. Nature 550(7675):265-269

Puerto-Galan L, Perez-Ruiz JM, Ferrandez J, Cano B, Naranjo B, Najera VA, Gonzalez M, Lindahl AM, Cejudo FJ (2013) Overoxidation of chloroplast 2-Cys peroxiredoxins: balancing toxic and signaling activities of hydrogen peroxide. Front Plant Sci 4:310

Radding CM (1981) Recombination activities of E. coli recA protein. Cell 25(1):3-4

Ranjit S, Dvornikov A, Holland DA, Reinhart GD, Jameson DM, Gratton E (2014) Application of three-photon excitation FCS to the study of protein oligomerization. J Phys Chem B 118(50):14627-14631

Ratcliffe AJ (2006) Inosine 5'-monophosphate dehydrogenase inhibitors for the treatment of autoimmune diseases. Curr Opin Drug Discov Devel 9(5):595-605

Ray H, Suau F, Vincent A, Dalla Venezia N (2009) Cell cycle regulation of the BRCA1/acetyl-CoA-carboxylase complex. Biochem Biophys Res Commun 378(3):615-619

Reinhart GD, Lardy HA (1980) Rat liver phosphofructokinase: kinetic and physiological ramifications of the aggregation behavior. Biochemistry 19(7):1491-1495

Ristow M, Carlqvist H, Hebinck J, Vorgerd M, Krone W, Pfeiffer A, Muller-Wieland D, Ostenson CG (1999a) Deficiency of phosphofructo-1-kinase/muscle subtype in humans is associated with impairment of insulin secretory oscillations. Diabetes $48(8)$ : $1557-1561$

Ristow M, Vorgerd M, Mohlig M, Schatz H, Pfeiffer A (1999b) Insulin resistance and impaired insulin secretion due to phosphofructo-1kinase-deficiency in humans. J Mol Med (Berl) 77(1):96-103

Rudolph FB (1994) The biochemistry and physiology of nucleotides. J Nutr 124(1 Suppl):124S-127S

Saad S, Cereghetti G, Feng Y, Picotti P, Peter M, Dechant R (2017) Reversible protein aggregation is a protective mechanism to ensure cell cycle restart after stress. Nat Cell Biol 19(10):1202-1213

Saarikangas J, Barral Y (2016) Protein aggregation as a mechanism of adaptive cellular responses. Curr Genet 62(4):711-724

Saccoccia F, Di Micco P, Boumis G, Brunori M, Koutris I, Miele AE, Morea V, Sriratana P, Williams DL, Bellelli A, Angelucci F (2012) Moonlighting by different stressors: crystal structure of the chaperone species of a 2-Cys peroxiredoxin. Structure 20(3):429-439

Saggerson D (2008) Malonyl-CoA, a key signaling molecule in mammalian cells. Annu Rev Nutr 28:253-272

Sawers G (1994) The hydrogenases and formate dehydrogenases of Escherichia coli. Antonie Van Leeuwenhoek 66(1-3):57-88

Schnitzler A, Olsen BB, Issinger OG, Niefind K (2014) The protein kinase CK2(Andante) holoenzyme structure supports proposed models of autoregulation and trans-autophosphorylation. J Mol Biol 426(9):1871-1882

Schoneberg T, Kloos M, Bruser A, Kirchberger J, Strater N (2013) Structure and allosteric regulation of eukaryotic 6-phosphofructokinases. Biol Chem 394(8):977-993

Schuchmann K, Muller V (2013) Direct and reversible hydrogenation of $\mathrm{CO} 2$ to formate by a bacterial carbon dioxide reductase. Science 342(6164):1382-1385

Schuchmann K, Muller V (2014) Autotrophy at the thermodynamic limit of life: a model for energy conservation in acetogenic bacteria. Nat Rev Microbiol 12(12):809-821

Schuchmann K, Vonck J, Muller V (2016) A bacterial hydrogendependent $\mathrm{CO} 2$ reductase forms filamentous structures. FEBS J 283(7):1311-1322

Schwock J, Kirchberger J, Edelmann A, Kriegel TM, Kopperschlager G (2004) Interaction of 6-phosphofructokinase with cytosolic proteins of Saccharomyces cerevisiae. Yeast 21(6):483-494

Seetoh WG, Chan DS, Matak-Vinkovic D, Abell C (2016) Mass Spectrometry Reveals Protein Kinase CK2 High-Order 
Oligomerization via the Circular and Linear Assembly. ACS Chem Biol 11(6):1511-1517

Selwood T, Jaffe EK (2012) Dynamic dissociating homo-oligomers and the control of protein function. Arch Biochem Biophys 519(2):131143

Selwood T, Tang L, Lawrence SH, Anokhina Y, Jaffe EK (2008) Kinetics and thermodynamics of the interchange of the morpheein forms of human porphobilinogen synthase. Biochemistry 47(10):3245-3257

Sener A, Malaisse WJ (1980) L-leucine and a nonmetabolized analogue activate pancreatic islet glutamate dehydrogenase. Nature 288(5787):187-189

Sewell BT, Berman MN, Meyers PR, Jandhyala D, Benedik MJ (2003) The cyanide degrading nitrilase from Pseudomonas stutzeri AK61 is a two-fold symmetric, 14-subunit spiral. Structure 11(11):14131422

Shah S, Sanchez J, Stewart A, Piperakis MM, Cosstick R, Nichols C, Park CK, Ma X, Wysocki V, Bitinaite J, Horton NC (2015) Probing the run-on oligomer of activated SgrAI bound to DNA. PLoS One 10(4):e0124783

Shashidharan P, Plaitakis A (2014) The discovery of human of GLUD2 glutamate dehydrogenase and its implications for cell function in health and disease. Neurochem Res 39(3):460-470

Shen Y, Tong L (2008) Structural evidence for direct interactions between the BRCT domains of human BRCA1 and a phospho-peptide from human ACC1. Biochemistry 47(21):5767-5773

Shen Q-J, Kassim H, Huang Y, Li H, Zhang J, Li G, Wang P-Y, Ye F, Liu J-L (2016) Filamentation of Metabolic Enzymes in Saccharomyces cerevisiae. J Genet Genomics 43:393-404

Sidrauski C, Cox JS, Walter P (1996) tRNA ligase is required for regulated mRNA splicing in the unfolded protein response. Cell 87(3): 405-413

Smith HQ, Smith TJ (2016) Identification of a novel activator of mammalian glutamate dehydrogenase. Biochemistry 55(47):6568-6576

Smith TS, Southan C, Ellington K, Campbell D, Tew DG, Debouck C (2001) Identification, genomic organization, and mRNA expression of LACTB, encoding a serine beta-lactamase-like protein with an amino-terminal transmembrane domain. Genomics 78(1-2):12-14

Stanley CA, Lieu YK, Hsu BY, Burlina AB, Greenberg CR, Hopwood NJ, Perlman K, Rich BH, Zammarchi E, Poncz M (1998) Hyperinsulinism and hyperammonemia in infants with regulatory mutations of the glutamate dehydrogenase gene. N Engl J Med 338(19):1352-1357

Stanley CA, Fang J, Kutyna K, Hsu BY, Ming JE, Glaser B, Poncz M (2000) Molecular basis and characterization of the hyperinsulinism/ hyperammonemia syndrome: predominance of mutations in exons 11 and 12 of the glutamate dehydrogenase gene. HI/HA Contributing Investigators. Diabetes 49(4):667-673

Stiede K, Miao W, Blanchette HS, Beysen C, Harriman G, Harwood HJ Jr, Kelley H, Kapeller R, Schmalbach T, Westlin WF (2017) Acetylcoenzyme A carboxylase inhibition reduces de novo lipogenesis in overweight male subjects: a randomized, double-blind, crossover study. Hepatology 66(2):324-334

Stoddard PR, Lynch EM, Farrell DP, Justman QA, Dosey AM, DiMaio F, Williams TA, Kollman JM, Murray AW, Garner EC (2019) Independent evolution of polymerization in the Actin ATPase clan regulates hexokinase activity. bioRxiv PPR:PPR84236

Strochlic TI, Stavrides KP, Thomas SV, Nicolas E, O'Reilly AM, Peterson JR (2014) Ack kinase regulates CTP synthase filaments during Drosophila oogenesis. EMBO Rep 15(11):1184-1191

Sun L, Wang H, Wang Z, He S, Chen S, Liao D, Wang L, Yan J, Liu W, Lei X, Wang X (2012) Mixed lineage kinase domain-like protein mediates necrosis signaling downstream of RIP3 kinase. Cell 148(12):213-227

Suresh HG, da Silveira Dos Santos AX, Kukulski W, Tyedmers J, Riezman H, Bukau B, Mogk A (2015) Prolonged starvation drives reversible sequestration of lipid biosynthetic enzymes and organelle reorganization in Saccharomyces cerevisiae. Mol Biol Cell 26(9): $1601-1615$

Svensson RU, Parker SJ, Eichner LJ, Kolar MJ, Wallace M, Brun SN, Lombardo PS, Van Nostrand JL, Hutchins A, Vera L, Gerken L, Greenwood J, Bhat S, Harriman G, Westlin WF, Harwood HJ Jr, Saghatelian A, Kapeller R, Metallo CM, Shaw RJ (2016) Inhibition of acetyl-CoA carboxylase suppresses fatty acid synthesis and tumor growth of non-small-cell lung cancer in preclinical models. Nat Med 22(10):1108-1119

Swinnen JV, Brusselmans K, Verhoeven G (2006) Increased lipogenesis in cancer cells: new players, novel targets. Curr Opin Clin Nutr Metab Care 9(4):358-365

Szeliga M, Obara-Michlewska M (2009) Glutamine in neoplastic cells: focus on the expression and roles of glutaminases. Neurochem Int 55(1-3):71-75

Tam AB, Koong AC, Niwa M (2014) Ire1 has distinct catalytic mechanisms for XBP1/HAC1 splicing and RIDD. Cell Rep 9(3):850-858

Tang L, Stith L, Jaffe EK (2005) Substrate-induced interconversion of protein quaternary structure isoforms. J Biol Chem 280(16):1578615793

Tang L, Breinig S, Stith L, Mischel A, Tannir J, Kokona B, Fairman R, Jaffe EK (2006) Single amino acid mutations alter the distribution of human porphobilinogen synthase quaternary structure isoforms (morpheeins). J Biol Chem 281(10):6682-6690

Tautz N, Kaluza K, Frey B, Jarsch M, Schmitz GG, Kessler C (1990) SgrAI, a novel class-II restriction endonuclease from Streptomyces griseus recognizing the octanucleotide sequence 5'-CR/CCGGYG3' [corrected]. Nucleic Acids Res 18(10):3087

Teixeira F, Tse E, Castro H, Makepeace KAT, Meinen BA, Borchers CH, Poole LB, Bardwell JC, Tomas AM, Southworth DR, Jakob U (2019) Chaperone activation and client binding of a 2-cysteine peroxiredoxin. Nat Commun 10(1):659

Telford JN, Lad PM, Hammes GG (1975) Electron microscope study of native and crosslinked rabbit muscle phosphofructokinase. Proc Natl Acad Sci U S A 72(8):3054-3056

Thanki CM, Sugden D, Thomas AJ, Bradford HF (1983) In vivo release from cerebral cortex of $[14 \mathrm{C}]$ glutamate synthesized from [U-14C]glutamine. J Neurochem 41(3):611-617

Thayer NH, Leverich CK, Fitzgibbon MP, Nelson ZW, Henderson KA, Gafken PR, Hsu JJ, Gottschling DE (2014) Identification of longlived proteins retained in cells undergoing repeated asymmetric divisions. Proc Natl Acad Sci U S A 111(39):14019-14026

Thomas EC, Gunter JH, Webster JA, Schieber NL, Oorschot V, Parton RG, Whitehead JP (2012) Different characteristics and nucleotide binding properties of inosine monophosphate dehydrogenase (IMPDH) isoforms. PLoS One 7(12):e51096

Thomas WC, Brooks FP 3rd, Burnim AA, Bacik JP, Stubbe J, Kaelber JT, Chen JZ, Ando N (2019) Convergent allostery in ribonucleotide reductase. Nat Commun 10(1):2653

Thuku RN, Weber BW, Varsani A, Sewell BT (2007) Post-translational cleavage of recombinantly expressed nitrilase from Rhodococcus rhodochrous J1 yields a stable, active helical form. FEBS J 274(8): 2099-2108

Thuku RN, Brady D, Benedik MJ, Sewell BT (2009) Microbial nitrilases: versatile, spiral forming, industrial enzymes. J Appl Microbiol 106(3):703-727

Tojo H, Horiike K, Shiga K, Nishina Y, Watari H, Yamano T (1985a) Self-association mode of a flavoenzyme D-amino acid oxidase from hog kidney. I. Analysis of apparent weight-average molecular weight data for the apoenzyme in terms of models. J Biol Chem 260(23):12607-12614

Tojo H, Horiike K, Shiga K, Nishina Y, Watari H, Yamano T (1985b) Self-association mode of a flavoenzyme D-amino acid oxidase from hog kidney. II. Stoichiometry of holoenzyme association and energetics of subunit association. J Biol Chem 260(23):12615-12621 
Tomkins GM, Yielding KL, Curran JF (1962) The influence of diethylstilbestrol and adenosine diphosphate on pyridine nucleotide coenzyme binding by glutamic dehydrogenase. J Biol Chem 237:1704 1708

Tong L (2013) Structure and function of biotin-dependent carboxylases. Cell Mol Life Sci 70(5):863-891

Trudel M, Van Genechten T, Meuth M (1984) Biochemical characterization of the hamster thy mutator gene and its revertants. J Biol Chem 259(4):2355-2359

Trujillo JL, Deal WC Jr (1977) Pig liver phosphofructokinase: asymmetry properties, proof of rapid association-dissociation equilibria, and effect of temperature and protein concentration on the equilibria. Biochemistry 16(14):3098-3104

Uptain SM, Lindquist S (2002) Prions as protein-based genetic elements. Annu Rev Microbiol 56:703-741

Upton JP, Wang L, Han D, Wang ES, Huskey NE, Lim L, Truitt M, McManus MT, Ruggero D, Goga A, Papa FR, Oakes SA (2012) IRE1alpha cleaves select microRNAs during ER stress to derepress translation of proapoptotic Caspase-2. Science 338(6108):818-822

Vagelos PR, Alberts AW, Martin DB (1962) Activation of acetyl-CoA carboxylase and associated alteration of sedimentation characteristics of the enzyme. Biochem Biophys Res Commun 8:4-8

Valero E, De Bonis S, Filhol O, Wade RH, Langowski J, Chambaz EM, Cochet C (1995) Quaternary structure of casein kinase 2. Characterization of multiple oligomeric states and relation with its catalytic activity. J Biol Chem 270(14):8345-8352

Vasu K, Nagaraja V (2013) Diverse functions of restriction-modification systems in addition to cellular defense. Microbiol Mol Biol Rev 77(1):53-72

Vejvoda V, Kaplan O, Bezouska K, Pompach P, Sulc M, Cantarella M, Benada O, Uhnakov B, Rinagelov A, Lutz-Wahl S, Fischer L, Kren V, Martınkova L (2008) Purification and characterization of a nitrilase from Fusarium solani O1. J Mol Catal B Enzym 50:99-106

Wakil SJ, Abu-Elheiga LA (2009) Fatty acid metabolism: target for metabolic syndrome. J Lipid Res 50(Suppl):S138-S143

Walter P, Ron D (2011) The unfolded protein response: from stress pathway to homeostatic regulation. Science 334(6059):1081-1086

Wang M, Kaufman RJ (2016) Protein misfolding in the endoplasmic reticulum as a conduit to human disease. Nature 529(7586):326-335

Wang F, Eric Knabe W, Li L, Jo I, Mani T, Roehm H, Oh K, Li J, Khanna M, Meroueh SO (2012) Design, synthesis, biochemical studies, cellular characterization, and structure-based computational studies of small molecules targeting the urokinase receptor. Bioorg Med Chem 20(15):4760-4773

Webb BA, Forouhar F, Szu FE, Seetharaman J, Tong L, Barber DL (2015) Structures of human phosphofructokinase-1 and atomic basis of cancer-associated mutations. Nature 523(7558):111-114

Webb BA, Dosey AM, Wittmann T, Kollman JM, Barber DL (2017) The glycolytic enzyme phosphofructokinase-1 assembles into filaments. J Cell Biol 216(8):2305-2313

Werner JN, Chen EY, Guberman JM, Zippilli AR, Irgon JJ, Gitai Z (2009) Quantitative genome-scale analysis of protein localization in an asymmetric bacterium. Proc Natl Acad Sci U S A 106(19):78587863

Wild K, Grafmuller R, Wagner E, Schulz GE (1997) Structure, catalysis and supramolecular assembly of adenylate kinase from maize. Eur J Biochem 250(2):326-331

Wood ZA, Poole LB, Hantgan RR, Karplus PA (2002) Dimers to doughnuts: redox-sensitive oligomerization of 2-cysteine peroxiredoxins. Biochemistry 41(17):5493-5504

Wood KM, Daniels LE, Halford SE (2005) Long-range communications between DNA sites by the dimeric restriction endonuclease SgrAI. J Mol Biol 350(2):240-253
Woodward JD, Weber BW, Scheffer MP, Benedik MJ, Hoenger A, Sewell BT (2008) Helical structure of unidirectionally shadowed metal replicas of cyanide hydratase from Gloeocercospora sorghi. J Struct Biol 161(2):111-119

Woodward JD, Trompetter I, Sewell BT, Piotrowski M (2018) Substrate specificity of plant nitrilase complexes is affected by their helical twist. Commun Biol 1:186

Wu B, Peisley A, Tetrault D, Li Z, Egelman EH, Magor KE, Walz T, Penczek PA, Hur S (2014) Molecular imprinting as a signalactivation mechanism of the viral RNA sensor RIG-I. Mol Cell 55(4):511-523

Yang Z, Matteson EL, Goronzy JJ, Weyand CM (2015) T-cell metabolism in autoimmune disease. Arthritis Res Ther 17:29

Yi W, Clark PM, Mason DE, Keenan MC, Hill C, Goddard WA 3rd, Peters EC, Driggers EM, Hsieh-Wilson LC (2012) Phosphofructokinase 1 glycosylation regulates cell growth and metabolism. Science 337(6097):975-980

Yielding KL, Tomkins GM (1961) An effect of L-leucine and other essential amino acids on the structure and activity of glutamic dehydrogenase. Proc Natl Acad Sci U S A 47:983-989

Yin Q, Fu TM, Li J, Wu H (2015) Structural biology of innate immunity. Annu Rev Immunol 33:393-416

Yoshida H, Matsui T, Yamamoto A, Okada T, Mori K (2001) XBP1 mRNA is induced by ATF6 and spliced by IRE1 in response to ER stress to produce a highly active transcription factor. Cell 107(7):881-891

Yu X, Jacobs SA, West SC, Ogawa T, Egelman EH (2001) Domain structure and dynamics in the helical filaments formed by RecA and Rad51 on DNA. Proc Natl Acad Sci U S A 98(15):8419-8424

Zeiri L, Reisler E (1978) Uncoupling of the catalytic activity and the polymerization of beef liver glutamate dehydrogenase. J Mol Biol 124(1):291-295

Zeng W, Sun L, Jiang X, Chen X, Hou F, Adhikari A, Xu M, Chen ZJ (2010) Reconstitution of the RIG-I pathway reveals a signaling role of unanchored polyubiquitin chains in innate immunity. Cell 141(2): 315-330

Zhang DW, Shao J, Lin J, Zhang N, Lu BJ, Lin SC, Dong MQ, Han J (2009) RIP3, an energy metabolism regulator that switches TNFinduced cell death from apoptosis to necrosis. Science 325(5938): 332-336

Zhang S, Ding K, Shen QJ, Zhao S, Liu JL (2018) Filamentation of asparagine synthetase in Saccharomyces cerevisiae. PLoS Genet 14(10):e1007737

Zhao J, Jitkaew S, Cai Z, Choksi S, Li Q, Luo J, Liu ZG (2012) Mixed lineage kinase domain-like is a key receptor interacting protein 3 downstream component of TNF-induced necrosis. Proc Natl Acad Sci U S A 109(14):5322-5327

Zhou J, Liu CY, Back SH, Clark RL, Peisach D, Xu Z, Kaufman RJ (2006) The crystal structure of human IRE1 luminal domain reveals a conserved dimerization interface required for activation of the unfolded protein response. Proc Natl Acad Sci U S A 103(39): $14343-14348$

Zimmermann AG, Gu JJ, Laliberte J, Mitchell BS (1998) Inosine-5'monophosphate dehydrogenase: regulation of expression and role in cellular proliferation and T lymphocyte activation. Prog Nucleic Acid Res Mol Biol 61:181-209

Publisher's note Springer Nature remains neutral with regard to jurisdictional claims in published maps and institutional affiliations. 